\title{
Testbeam Measurements with Pixel Sensors for the ATLAS Insertable b-Layer Project
}

\author{
Dissertation
}

zur Erlangung des mathematisch-naturwissenschaftlichen Doktorgrades

"Doctor rerum naturalium"

der Georg-August-Universität Göttingen

\author{
im Promotionsprogramm ProPhys \\ der Georg-August University School of Science (GAUSS) \\ vorgelegt von \\ Matthias George \\ aus Kassel
}

Göttingen, 2014

II.Physik-UniGö-Diss-2014/01 



\section{Betreuungsausschuss:}

PD Dr. J. Große-Knetter, II. Physikalisches Institut, Georg-August-Universität Göttingen

Prof. Dr. A. Quadt, II. Physikalisches Institut, Georg-August-Universität Göttingen

Prof. Dr. A. Frey, II. Physikalisches Institut, Georg-August-Universität Göttingen

\section{Mitglieder der Prüfungskommission:}

Referent: PD Dr. J. Große-Knetter, II. Physikalisches Institut, Georg-August-Universität Göttingen

Korreferent: Prof. Dr. A. Quadt, II. Physikalisches Institut, Georg-August-Universität Göttingen

Weitere Mitglieder der Prüfungskommission:

Prof. Dr. A. Frey, II. Physikalisches Institut, Georg-August-Universität Göttingen

Prof. Dr. W. Kollatschny, Institut für Astrophysik, Georg-August-Universität Göttingen

Prof. Dr. M. Seibt, IV. Physikalisches Institut, Georg-August-Universität Göttingen

Prof. Dr. J. Enderlein, III. Physikalisches Institut, Georg-August-Universität Göttingen 



\section{Contents}

1 Introduction 1

2 The Large Hadron Collider and the ATLAS Experiment 3

2.1 The Large Hadron Collider . . . . . . . . . . . . . . . . . . . . . . . . 3

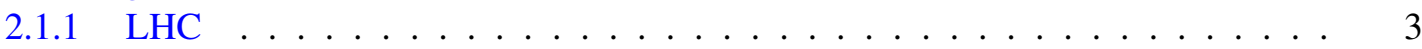

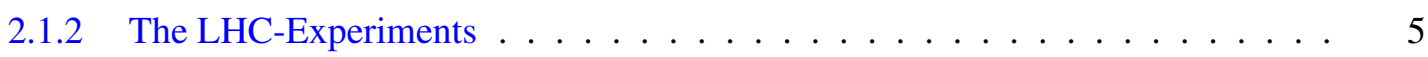

2.2 Physics Goals at the LHC - motivation for further upgrades . . . . . . . . . . . . 6

2.2.1 The Higgs Measurement Programme . . . . . . . . . . . . . . . . 6

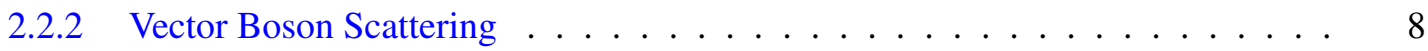

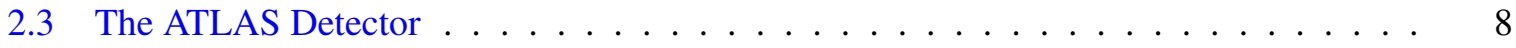

2.3 .1 Detector Subsystems . . . . . . . . . . . . . . . . . . . . . 9

2.3 .2 Inner Detector . . . . . . . . . . . . . . . . . . . . . . . . . 14

3 The ATLAS Pixel Detector 17

3.1 Silicon Detector Basics . . . . . . . . . . . . . . . . . . . . . . . . 17

3.1.1 Charged Particles in Matter . . . . . . . . . . . . . . . . . . 17

3.1.2 Energy Loss of Photons in Matter . . . . . . . . . . . . . . . . . . . . . 19

3.1 .3 Energy Bands . . . . . . . . . . . . . . . . . . . . 20

3.1 .4 Doping of Semiconductors . . . . . . . . . . . . . . . . . 21

3.1.5 Silicon as detector: $\mathrm{p}$-n-junction . . . . . . . . . . . . . . . . . 21

3.1 .6 Multiple Scattering . . . . . . . . . . . . . . . . . . . . . . 23

3.1.7 Radiation Damage in Silicon Detectors . . . . . . . . . . . . . . . . . 23

3.2 Current ATLAS Pixel Detector Layout . . . . . . . . . . . . . . . . . . . . . . . 24

3.2 .1 Module Layout . . . . . . . . . . . . . . . . . . . . . . . . 24

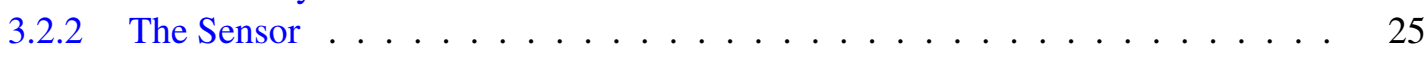

3.2 .3 The Front-End Chip . . . . . . . . . . . . . . . . . . 26

4 Upgrades of the ATLAS Experiment - The Insertable b-Layer and beyond 31

4.1 Upgrades of the ATLAS Experiment . . . . . . . . . . . . . . . . . 31

4.1 .1 The Insertable b-Layer . . . . . . . . . . . . . . . . . . . . . 31

4.1 .2 Upgrades for the High-Lumi LHC . . . . . . . . . . . . . . . . . . . . . . 34

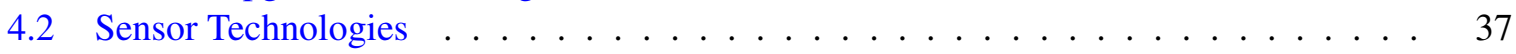

4.2 .13 3D Pixel Sensors . . . . . . . . . . . . . . . . . . . . . 38

$4.2 .2 \quad$ Planar Pixel Sensors . . . . . . . . . . . . . . . . . . . . . . . . . . . . 40

4.2 .3 Diamond Beam Monitor . . . . . . . . . . . . . . . . . . . . 42

4.2.4 Technology Choice . . . . . . . . . . . . . . . . . . 43

5 Testbeam Setup $\quad 45$

5.1 The ATLAS Pixel Testbeam setup . . . . . . . . . . . . . . . . . 45

5.1 .1 Mimosa Sensors . . . . . . . . . . . . . . . . . . 45 
5.1 .2 Devices Under Test . . . . . . . . . . . . . . . . . . . . . . . . . . . 47

5.1 .3 Data Acquisition Hardware . . . . . . . . . . . . . . . . . . 47

5.1 .4 The EUDAQ Package $\ldots \ldots \ldots \ldots \ldots \ldots$

5.2 Testbeam Facilities $\ldots \ldots \ldots \ldots \ldots \ldots$

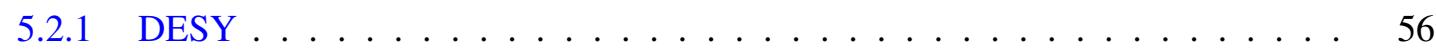

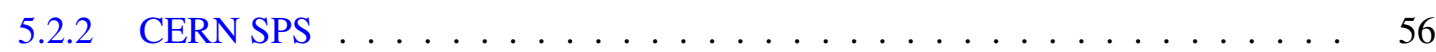

6 Testbeam Track Reconstruction and Analysis Tools $\quad 61$

6.1 Track Reconstruction - The ILC Framework . . . . . . . . . . . . . . . . . . . . 61

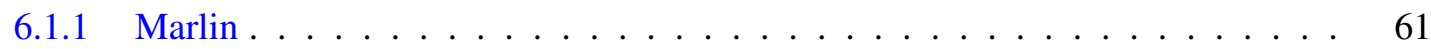

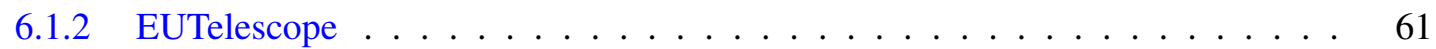

6.2 Analysis Tools - TBmon . . . . . . . . . . . . . . . . . . 71

6.2.1 Eventbuilders and Preprocessing . . . . . . . . . . . . . . . . 73

6.2.2 Analysis Processors . . . . . . . . . . . . . . . . . . . 78

6.2.3 Ongoing Developments - TBmon II . . . . . . . . . . . . . . . . 88

7 Testbeam Analysis $\quad 93$

$7.1 \quad$ IBL Device Analysis . . . . . . . . . . . . . . . . . . . . . . . . . . . 93

7.2 n-in-p Sensors . . . . . . . . . . . . . . . . . . . . . . . . . . . . . 93

7.3 IBL Analysis . . . . . . . . . . . . . . . . . . . . . . . . . . . 94

7.4 Planar Pixel Sensors - Results _ . . . . . . . . . . . . . . . . . . . . . . 96

7.4.1 Slim-Edge Designs . . . . . . . . . . . . . . . . . . . . . . . . . . 99

7.5 3D Pixel Sensors - Results . . . . . . . . . . . . . . . . . . . . . . . 109

7.5 .1 Double Sided Designs . . . . . . . . . . . . . . . . . . . . . . . 109

7.5 .2 Full 3D Sensors . . . . . . . . . . . . . . . . . . . . . . . . . 112

7.5 .3 IBL Sensors - Summary . . . . . . . . . . . . . . . . . . 117

7.6 IBL Production Modules . . . . . . . . . . . . . . . . . . . . . . . . . . . . . . . 119

7.7 Large Sensors for HL-LHC . . . . . . . . . . . . . . . . . . . . . . 124

8 Summary and Outlook $\quad 127$

8.1 Summary . . . . . . . . . . . . . . . . . . . . . . . . . 127

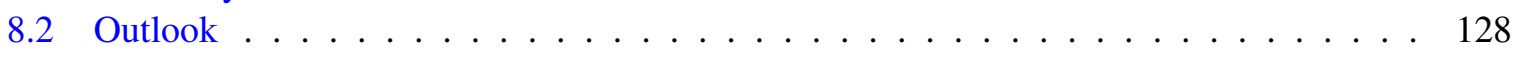

$\begin{array}{ll}\text { A Useful information } & 131\end{array}$

A.1 Testbeam Dictionary . . . . . . . . . . . . . . . . . . . . . . . . . . 131

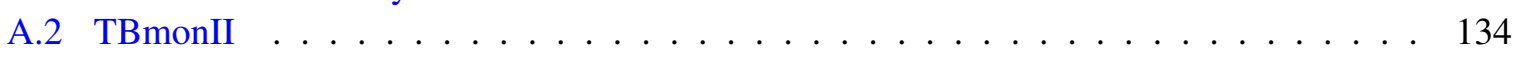

$\begin{array}{ll}\text { Bibliography } & 135\end{array}$

$\begin{array}{ll}\text { List of Figures } & 141\end{array}$

List of Tables $\quad 153$ 


\section{Chapter 1}

\section{Introduction}

In the search for theories to explain big parts of our surrounding world, the Standard Model of particle physics [1-4] is amongst the most successful ones. The model describes all elementary particles that were found by measurements in the last decades and the interactions between them. The level of agreement between Standard Model predictions and recent measurements and discoveries is certainly an extraordinary success.

One of the missing pieces in the puzzle of nature on the elementary particle scale, the mechanism which gives mass to particles, has been revealed by the ATLAS and CMS experiments at the Large Hadron Collider (LHC) in the year 2012 with the announcement of the discovery of a Higgs boson [5]. This discovery did not mark the end of particle physics research, but opens the door to new fields of research. There is still a long way to go and even particles that were discovered in the past are not analysed in all their properties yet. Most of the properties can only be measured indirectly through the analysis of decay products, which makes an increase in measurement precision towards the technical limits vital for further analyses.

This hunt for higher precision and also search for the unknown is performed with the Large Hadron Collider. To gain access to extremely rare decays and also to possible new particles, the machine is currently being upgraded to higher beam energies and luminosities. Further upgrades are planned for the future, where especially the plan for the next decade is set, going towards the High Luminosity Large Hadron Collider (HL-LHC). Due to the fact that the experiments have to keep pace with the new machine specifications, the experiments have to use the scheduled long downtimes of the machine for their own upgrades [6].

Within the ATLAS experiment, the current long shutdown is used to perform an upgrade on the innermost part of the experiment, the pixel detector. This tracking part of the experiment is currently equipped with pixelated silicon sensors, organized in three barrel layers and three end cap disks on either side. To cope with the higher instantaneous luminosity and for compensation of radiation damages due to past and near future running time of the experiment, a new fourth pixel detector layer is inserted into the existing system. This additional pixel layer is called "Insertable b-Layer" (IBL), which is described in detail in chapter 4 .

The IBL is a detector system, based on silicon pixel sensors. Due to the smaller radius, compared to all other detectors of the ATLAS experiment, it has to be more radiation tolerant, than e.g. the current pixel layers. Furthermore, the pixel size has to shrink to cope with the expected higher particle flux. Since a smaller pixel size also requires new front-end (FE) electronics, the new FE-chip generation I4 was developed for IBL sensors, having a pixel pitch of $250 \mu \mathrm{m} \times 50 \mu \mathrm{m}$. During the planning phase for the IBL upgrade, three different sensor technologies were competing, namely 3D silicon sensors, planar silicon sensors and diamond sensors. For each of the two kinds of silicon sensor approaches, different design alternatives were taken into account as IBL candidates. To find the best sensor for the IBL upgrade, a series of measurements and tests were performed. Beside lab tests, one of the most important instruments to investigate the performance of a prototype module under realistic conditions are 
testbeam measurements. Over the last three years, a series of testbeam measurements was performed using the facilities at CERN (Geneva) and DESY (Hamburg). Because of the fact that the timescale for the IBL project was shortened by about three years compared to the original schedule, diamond sensors faded out as sensor candidate, due to a lack of available sensors. Thus, the competing technologies for the IBL project were 3D and planar silicon devices.

Chapter 2 gives a general overview on the LHC, the motivations for future upgrades and the ATLAS experiment. Chapter 3 introduces the general functionality of semiconductors, especially silicon as sensitive material, with a focus on the effects playing a role for the testbeam measurements performed in the context of this thesis. In addition, as reference for the IBL developments, an overview on the current ATLAS pixel detector is given.

In chapter 4 the Insertable b-Layer project is described in detail, presenting the requirements that were made for the new pixel modules. Here the competing sensor technologies are presented, also giving a short insight into the utilisation of diamond sensors in the context of the current upgrade. Chapter 5 gives a summary of the setup that was used and adapted by the ATLAS pixel community, to perform the testbeam measurements for the IBL investigations, but also for developments by the different ATLAS pixel sensor groups. In chapter 6 the track reconstruction and device analysis tools that were developed in the context of these measurements are presented. This chapter also briefly summarizes ongoing software developments towards a new testbeam analysis tool. The chronological development and corresponding testbeam results of the IBL sensor candidates is given in chapter 7. For the final sensor decision this set of results was used as a major input. Finally, in chapter 8 , a summary and an outlook are given. 


\section{Chapter 2}

\section{The Large Hadron Collider and the ATLAS Experiment}

\subsection{The Large Hadron Collider}

\subsubsection{LHC}

To gain access to the parameter space of new physics within and beyond the standard model, colliders with higher and higher energies were built. The most recent high energy particle collider in this series is the Large Hadron Collider (LHC) at the European Organization for Nuclear Research, CERN. The experimental setup gives access to an unprecedented energy range for the resulting particles, as well as the possibility for precision measurements, using the LHC-experiments. An overview sketch of the LHC including the experiments can be seen in figure 2.1. The LHC is located in a tunnel close to Geneva [7]. With a circumference of about $27 \mathrm{~km}$, this accelerator is one of the biggest machines humans ever built. Its current design is aiming towards a centre of mass energy for colliding protons of $14 \mathrm{TeV}$. Beside the proton-proton measurement programme, proton-ion and ion-ion collisions are performed. To be able to achieve such high particle energies in collider operation, a number of 1232 superconducting niobiumtitanium dipole magnets with a field strength of up to $8.4 \mathrm{~T}$ had to be built, which require an operation at $1.9 \mathrm{~K}$. Another challenge for the designers of the accelerator was to construct a combined system of cryostat, beampipe and magnet in such a way, that the magnetic field lines direct the counter-rotating proton beams within the beam pipes using one iron yoke. A sketch of a cryo-dipole is shown in figure 2.2.

Because superconducting magnets providing such strong fields can only be operated within certain parameters, the LHC can not accelerate protons from rest to $7 \mathrm{TeV}$. The minimum energy when entering the LHC ring is $450 \mathrm{GeV}$. To bring the protons to injection energy, they are pre-accelerated in a chain of accelerators that were already existing at CERN. The protons are gained from hydrogen, which is being ionized and accelerated to $50 \mathrm{MeV}$ in the LINAC II. From there, protons are injected into the Booster, where they are brought to $1.4 \mathrm{GeV}$ and fed into the Proton Synchrotron (PS), which accelerates them to an energy of $25 \mathrm{GeV}$. The last pre-acceleration step is forwarding the protons to the Super Proton Synchrotron (SPS), where they reach the aimed for energy of $450 \mathrm{GeV}$, which allows injection to the LHC. Beside the usage as pre-accelerator for the LHC, SPS also provides testbeams for several beamlines. More details on the SPS as a testbeam facility can be found in section 5.2.2. In ion acceleration mode, ions are being accelerated starting at the LINAC III accelerator and the Low Energy Ion Ring (LEIR) and further on to the Proton Synchrotron (PS).

Since the search for new physics requests not only high centre of mass energies, but also new physics events are expected to occur rarely, the LHC design aimed for unprecedented luminosities of approximately $L \sim 10^{34} \mathrm{~cm}^{-2} \mathrm{~s}^{-1}$ to countervail this problem. These design values are realized by colliding packages of $\sim 10^{11}$ protons at a collision rate of $40 \mathrm{MHz}$. The machine design is as close to the technological limits, that even small beam disturbances on short timescales, can quench a magnet [8]. As a consequence, the 
collimation of beams has to be very good, as well as the monitoring of the beam quality. Several detector systems monitor the beam conditions permanently and as soon as a certain threshold (depending on the system: number of counts/measured signal) is reached, the beams are dumped. Not only that extremely fast and strong magnets (a whole dumping process only requires $86 \mu \mathrm{s}$ ) are required to realize such a beam dump, but also the facilities in the beam dump area had to be designed for these energies. For the LHC each beam dump at design values receives enough energy to e.g. melt a $500 \mathrm{~kg}$ copper block. For perfect beam conditions, a very high quality vacuum on the order of $10^{-11} \mathrm{mbar}$ is necessary, since even single dust particles can cause enough interaction with the beam (called UFO events) to cause a dump.

\section{CERN Accelerator Complex}

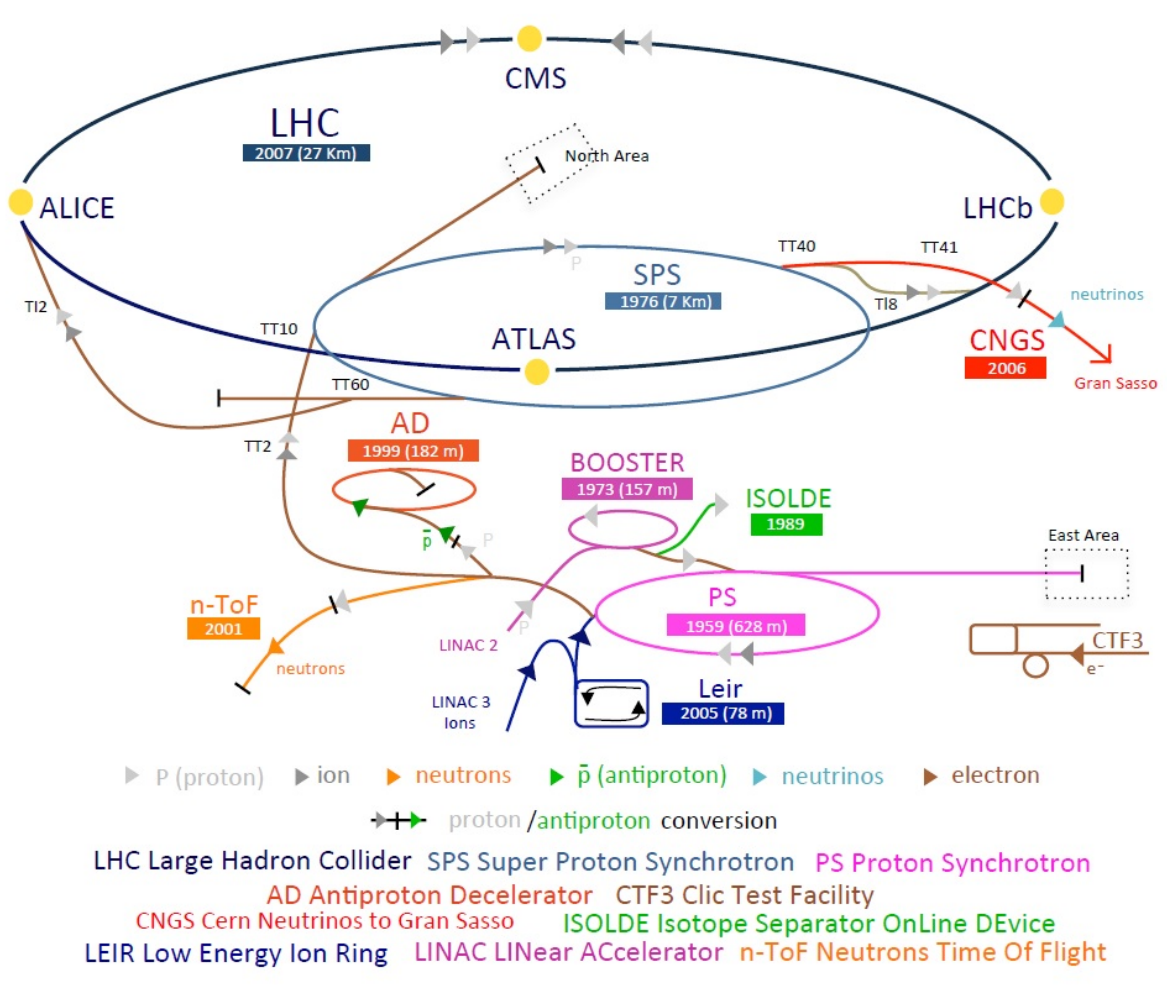

Figure 2.1: Schematic of the CERN accelerator system and the positions of the four LHC experiments (figure redrawn by [9]).

Currently the LHC is undergoing an upgrade, called "long shutdown 1" (LS1). Due to some technical difficulties with the magnet system, the LHC was limited to a centre of mass energy of $7 \mathrm{TeV}$ from its first days of operation in 2009 until 2012 [10]. After a small machine upgrade, the energy could be increased to $8 \mathrm{TeV}$ in 2012. During the scheduled LS1 phase, which started in spring 2013 and is supposed to last until end of 2014, the machine will be upgraded to its design energy for the next data taking period, starting in 2015. This shutdown period is also used by the pre-accelerator groups for maintenance work, as well as for some preparatory work towards future upgrades ("HL-LHC"). 


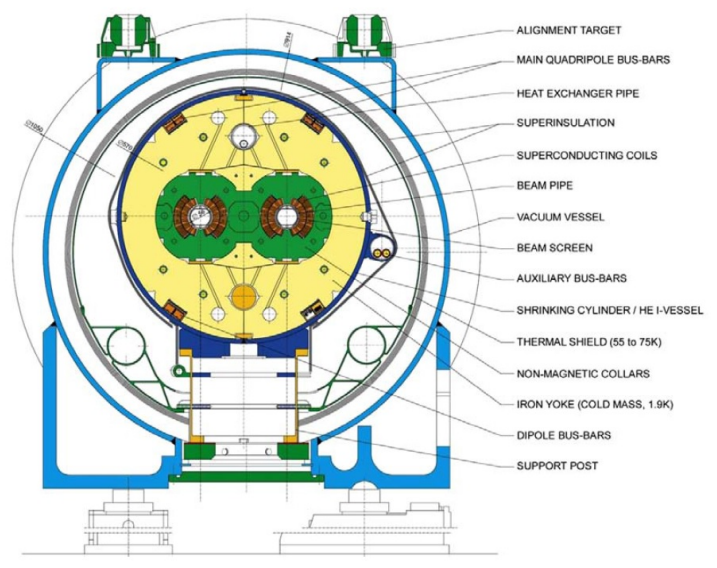

Figure 2.2: Cross section of a cryo-dipole at the Large Hadron Collider [7].

\subsubsection{The LHC-Experiments}

The LHC design includes four interaction regions, where particle beams cross. Covering the interaction points, four different experiments were built, each with its own design and own measurement targets. With ATLAS [11] and CMS [12], two multi purpose experiments are performing, amongst others, Higgs physics and searches for indications for supersymmetry and dark matter candidates. Both experiments cover almost the whole solid angle range. More details on the ATLAS experiment are given in section 2.3. The LHCb experiment is located at the third interaction point and specializes in the search for rare decays of charm and beauty hadrons [13]. Due to this specialization, the experiment does not provide a symmetric coverage around the interaction point, but is arranged for measurements in the forward direction. The ALICE detector is covering the fourth interaction region of the LHC [14]. This experiment is designed for the ion collision phases of the LHC, aiming towards investigating the physics of matter which is strongly interacting at extreme temperatures and energy densities and performing a search for quark gluon plasma signatures.

During the time that the LHC machine is upgraded, the experiments perform their own upgrades as well. During the long shutdown 1 phase also the experiments perform several upgrades [6]. The ATLAS experiment will be equipped with a new fourth pixel layer (see also 4.1.1) and new services will be installed for the pixel system, called "new service quarter panels" (nSQP). This project requires also the installation of a new, smaller beam pipe. For a more stable operation also some calorimeter power supplies will be renewed and the magnets' cryogenic system will have a higher operational stability and redundancy. The CMS experiment will upgrade the muon system to increase trigger efficiency, the calorimeter system will be upgraded in the light detection systems and the general power supply will be made more reliable by introducing more uninterruptible power supplies. For the LHCb experiment the upgrade includes the installation of a new beam pipe, as well as a replacement of the protection between the magnet coils and support brackets. Beside that, a lot of preparation work for upgrades planned during the next long shutdown is performed. The ALICE experiment will be equipped with an electromagnetic calorimeter extension, the transition radiation detector will be completed by adding the modules whose installation was postponed and some aged electrical and cooling infrastructure will be replaced.

Beside the four big experiments, several more are installed around the LHC. Examples are MoEDAL [15], searching for magnetic monopoles being produced at the LHC and TOTEM [16], providing high pre- 
cision proton-proton cross-section measurements and studying diffractive and elastic cross-sections. Another international experiment is LHCf [17], investigating the very forward region $140 \mathrm{~m}$ away from a particle interaction point. In this region a search for extremely high energetic neutral particles is performed, to calibrate models for very high energetic cosmic rays.

\subsection{Physics Goals at the LHC - motivation for further upgrades}

For the near future the LHC upgrade schedule is set, where part of the programme is to install the Insertable b-Layer as fourth pixel layer in ATLAS. The current schedule foresees a long shutdown (LS3) starting in $\sim 2023$, which is supposed to last about 30 months. During this shutdown the plan is to increase to instantaneous luminosity to $\sim 5 \times 10^{34} \mathrm{~cm}^{-2} \mathrm{~s}^{-1}$. The target for the integrated luminosity after 10 years of operation at the upgraded machine values is $3000 \mathrm{fb}^{-1}$. For comparison: until end of 2012 around $30 \mathrm{fb}^{-1}$ were collected. In the following a short overview over a selection of physics goals for the ATLAS experiment is presented, that gives the motivation for future upgrade plans [18].

\subsubsection{The Higgs Measurement Programme}

As a common effort, ATLAS and CMS presented the discovery of a Higgs boson in July 2012 [5]. For the theoretical prediction of this particle the Nobel prize was awarded, which was only possible due to the results of the two experiments. This discovery opened a whole new list of questions and tasks for the Higgs measurement programme. To clarify the nature of this particle, its quantum numbers have to be measured and couplings to other particles have to be investigated. With a mass of $\sim 126 \mathrm{GeV}$, this Higgs boson fits perfectly the energy range that can be scanned with the LHC. Couplings of the Higgs to other particles are of special interest, since the Standard Model predicts a set of coupling constants, where a significant deviation would be a hint for new physics. Particularly several SUSY models predict deviating coupling constants. Thus, this measurement can either open the door for models beyond the Standard Model or be used to set further limits. The same is true for the search for heavier Higgs particles, which would be an indication for new physics. Since most of the interesting processes are extremely rare, a higher integrated luminosity is of big interest. For the Higgs discovery publication, around 1500 Higgs events were used by the ATLAS and CMS collaborations. After upgrading the machine to HL-LHC, the expectation is that after 10 years of operation, in total around 100 million Higgs are produced (where a certain fraction, on the order of $10 \%$, is supposed to be reconstructed). Hence the upgrade can give access to the measurement of some of those events that occur very rarely.

As an example, figure 2.3 (left) shows the $t \bar{t} H$ mass distribution for the $H \longrightarrow \gamma \gamma$ channel, which allows access to measurements of the top Yukawa coupling. Even with $3000 \mathrm{fb}^{-1}$ just few hundred events are expected in this channel. Figure 2.3 (right) shows the expected $H \longrightarrow \mu \mu$ inclusive invariant mass distribution. As is visible in the distribution, the background for this channel is huge, but since the signal peak is quite sharp, a measurement significance of $6 \sigma$ for the channel is expected. In the exclusive study the simulation predicts around 30 events, but again with high purity. A detailed overview over the sensitivity improvements with upgrading the luminosity for all channels is given in [18].

Another topic of big interest is the measurement of the Higgs self coupling, as shown in figure $2.4 \mathrm{c}$ ). Since the identified Higgs boson has a mass of around $126 \mathrm{GeV}$, according to the Standard Model the mass has to be explained by a self coupling of the Higgs. The most promising channel in terms of signal purity combined with the number of expected events at the LHC is $H H \longrightarrow b \bar{b} \gamma \gamma$, shown in figure 2.5. Again this channel is very rare, which in numbers means that using the whole statistics of HL-LHC around 260 events are expected. Also in this channel the simulated background is huge, which means 

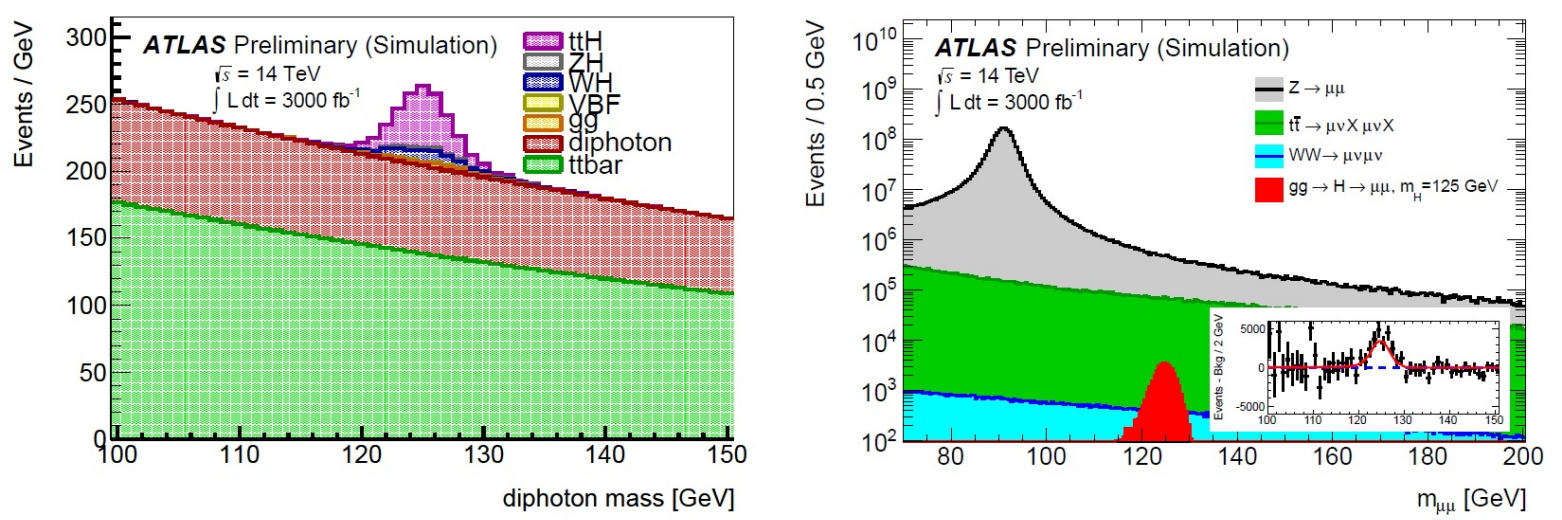

Figure 2.3: Left: Simulated invariant mass distribution in the $t \bar{t} H, H \longrightarrow \gamma \gamma$ channel for the estimated full HLLHC luminosity of $3000 \mathrm{fb}^{-1}$. Right: Simulated invariant mass distribution for the inclusive $H \longrightarrow \mu \mu$ channel, again for a total luminosity of $3000 \mathrm{fb}^{-1}$. In red the expected signal is shown, the other colours describe the background [18].

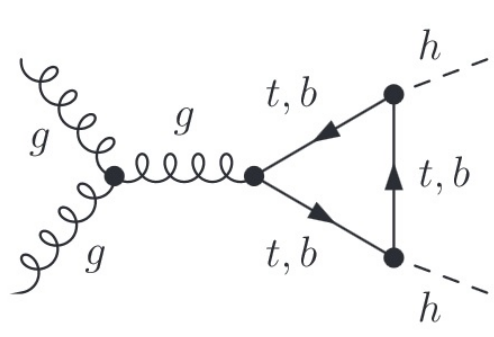

(a)

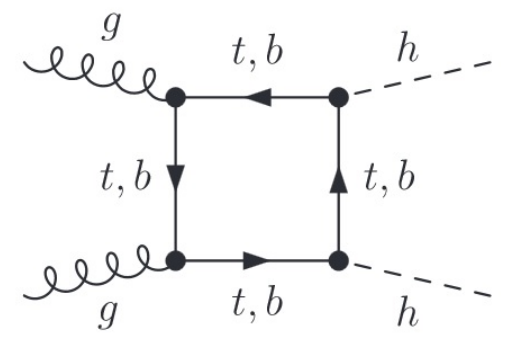

(b)

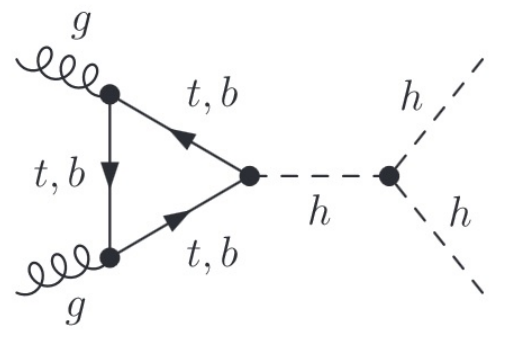

(c)

Figure 2.4: Some example Feynman diagrams, which contribute to $p p \longrightarrow h h+X$. While graphs a) and b) contribute to the background in a Higgs self coupling measurement, graph c) is the process of interest [19].

on the order of several $10^{5} \mathrm{fb}$, compared to around $0.087 \mathrm{fb}$ for the desired signal. Following the cut flow the expectation is that around 15 events can be obtained. Thus, if there is a chance at all to measure the Higgs self coupling based on facilities which already exist and can be upgraded towards certain measurement goals, it is with the HL-LHC.

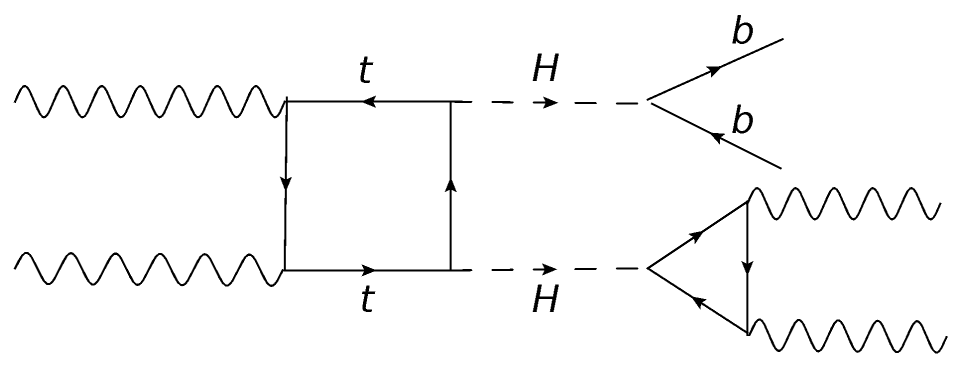

Figure 2.5: Feynman diagram for the $H H \longrightarrow b \bar{b} \gamma \gamma$ channel, which should be investigated at the HL-LHC. 


\begin{tabular}{|l|c|c|}
\hline model & $300 \mathrm{fb}^{-1}$ & $3000 \mathrm{fb}^{-1}$ \\
\hline \hline $\begin{array}{l}\mathrm{m}_{\text {resonance }}=0.5 \mathrm{TeV} \\
g=1.0\end{array}$ & $2.4 \sigma$ & $7.5 \sigma$ \\
\hline $\begin{array}{l}\mathrm{m}_{\text {resonance }}=1 \mathrm{TeV} \\
g=1.75\end{array}$ & $1.7 \sigma$ & $5.5 \sigma$ \\
\hline $\begin{array}{l}\mathrm{m}_{\text {resonance }}=1 \mathrm{TeV} \\
g=2.5\end{array}$ & $3.0 \sigma$ & $9.4 \sigma$ \\
\hline
\end{tabular}

Table 2.1: Comparison of the expexted increase of significance for three exemplary resonance masses and coupling values $g$. The expectation is to have collected an integrated luminosity of $300 \mathrm{fb}^{-1}$ until the long shutdown for the HL-LHC upgrade begins (left column). After additional 10 years of running the HL-LHC $3000 \mathrm{fb}^{-1}$ are expected in total (right column).

\subsubsection{Vector Boson Scattering}

In the Standard Model the cross section of vector boson scattering (VBS) would rise infinitely with energy from the $\mathrm{TeV}$ scale on, if the Higgs did not moderate the process. Other models predict different processes to moderate the process. Thus, a measurement of the VBS versus energy is of importance to either consolidate the Standard model or to find indications for new physics. An exemplary Feynman diagram for a VBS process is shown in figure 2.6. Here the measurement of the cross section for the process $p p \longrightarrow Z Z+2 j \longrightarrow l l l l+2 j$ is of interest, since with an integrated luminosity of $3000 \mathrm{fb}^{-1} \mathrm{a}$ statistical precision of $10 \%$ is expected. In case of any deviations from the Standard Model prediction, the sensitivity to anomalous VBS signals at a centre of mass energy of $14 \mathrm{TeV}$ could cross the discovery limit. As example, the increase of significance for three exemplary resonance masses and coupling values $g$, compared to before HL-LHC time ${ }^{1}$, is given in table 2.1.

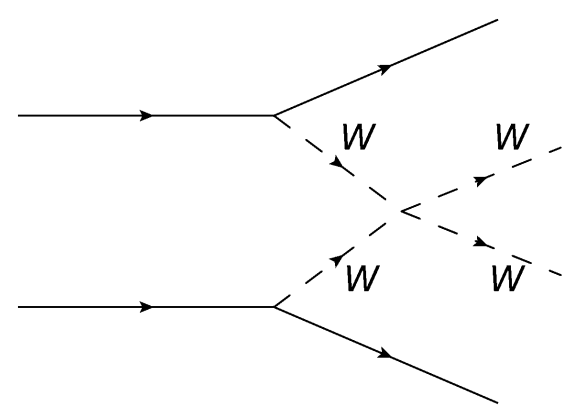

Figure 2.6: Exemplary Feynman diagram for a vector boson scattering process, which should be investigated at the HL-LHC.

\subsection{The ATLAS Detector}

The ATLAS ${ }^{2}$ experiment [11] is installed at one of the four interaction points of the LHC (see section 2.1.1). This experiment was designed as a multi purpose detector, where one main focus was and still is the Higgs measurement programme, as well as the search for physics beyond the standard model (see previous section). The ATLAS detector is composed of three main concentric subparts, which can

\footnotetext{
${ }^{1}$ with an expected integrated luminosity of $300 \mathrm{fb}^{-1}$

${ }^{2}$ A Toroidal LHC ApparatuS
} 
be seen in figure 2.7. Each of the parts is described in more detail in the following sections. From the outer parts towards the interaction point they are:

- the muon spectrometer, designed to measure the trajectory and momentum of muons

- the calorimeter system, measuring the hadronic and electromagnetic energy deposition of particles originating from the interaction point

- the Inner Detector, which is designed to measure the momentum and trajectories of charged particles

- the magnet system, which provides very high field strengths in different orientations to bent the particle tracks for momentum measurements

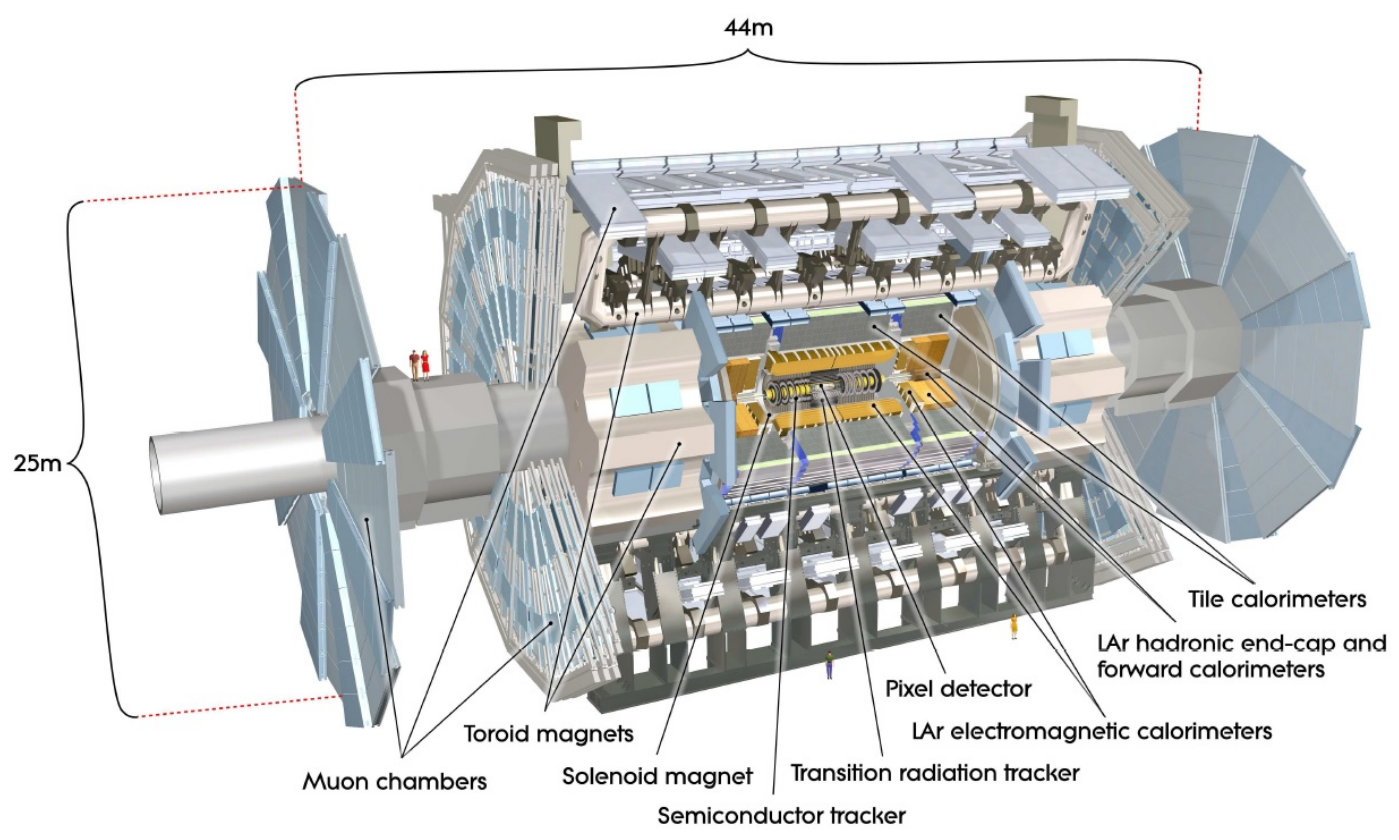

Figure 2.7: Schematic layout of the ATLAS Detector, indicating its dimensions and the different subsystems. A description of the detector systems can be found in section 2.3.1 and [11].

\subsubsection{Detector Subsystems}

\section{The Muon Spectrometer}

The ATLAS muon spectrometer provides two functionalities, which are a fast triggering of muons originating from the interaction point and the measurement of the muon momentum with high precision, using the track curvature. An overview of the whole muon system can be seen in figure 2.8. As shown in the figure, the huge magnet coils of the ATLAS toroid system are mounted inside the muon system. To fulfil the different tasks of triggering and tracking in different environments, four kinds of detectors are used in the muon spectrometer.

For the fast triggering part, resistive plate chambers (RPCs) and thin gap chambers (TGCs) were installed. The RPCs are mounted in the barrel part of the spectrometer, where they are operated in 
avalanche mode. This system provides a measured timing resolution of below 2 ns and therefore an excellent trigger capability. In the end caps a TGC system is used, which is a multi wire chamber, operated in saturation mode, providing a trigger resolution of better than $4 \mathrm{~ns}$.

The tracking and momentum measurement part of the spectrometer is made out of monitored drift tubes (MDTs) and cathode strip chambers (CSCs). The MDTs are gas filled aluminium tubes, providing a multi layer spatial resolution of $50 \mu \mathrm{m}$. They cover the pseudorapidity region of $|\eta|<2$. In the region between $2<|\eta|<2.7$ the system is constructed out of CSCs. These are multi wire proportional chambers with a resolution of $60 \mu \mathrm{m}$.

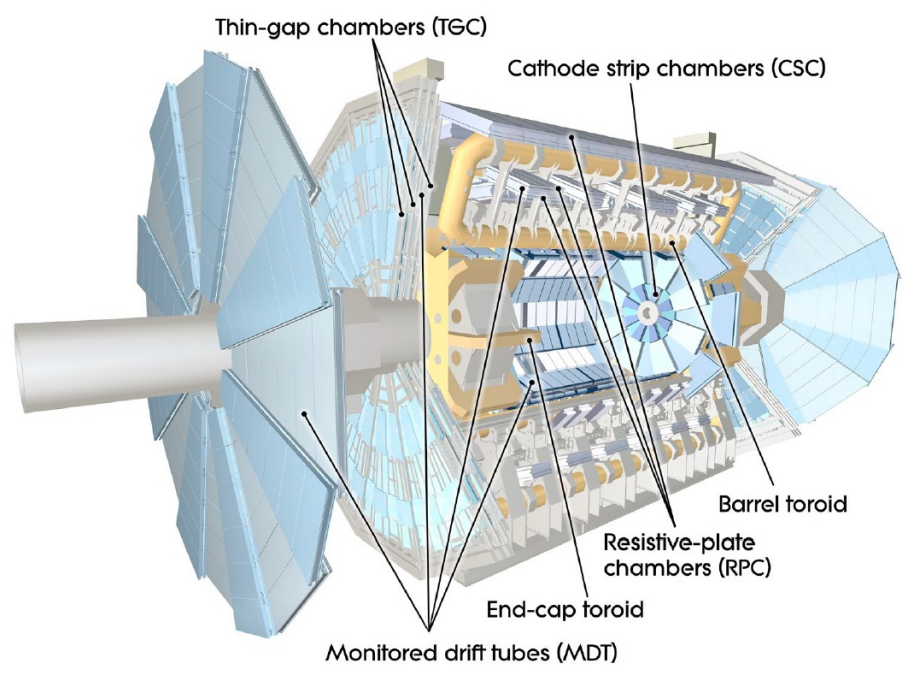

Figure 2.8: Schematic overview for the different parts of the ATLAS muon system [11].

\section{ATLAS Magnet System}

The ATLAS magnet system is made out of a barrel toroid, two end cap toroids and and an inner solenoid $[20,21]$. To achieve the required magnetic field strength, superconducting coils are used in the whole system.

One of the most characteristic shapes of the ATLAS experiment is given by the barrel toroid system with its eight air-core coils, assembled symmetrically around the beam axis. With a size of $25 \mathrm{x} 5 \mathrm{~m}^{2}$ the barrel toroid coils are among the largest parts of the ATLAS experiment. To keep the coils in their superconducting state, each coil is equipped with its own cryostat and kept in a vacuum vessel for thermal insulation, keeping them at their operating temperature of $4.5 \mathrm{~K}$. The operating current is specified as $20.5 \mathrm{kA}$, resulting in a field of $3.85 \mathrm{~T}$. A sketch of the ATLAS toroid system is shown in figure 2.9. The end cap toroid system consists of two almost identical systems, installed on either side of the ATLAS experiment. Each system has a size of $8.5 \times 5 \mathrm{~m}^{2}$ and hosts eight coils. The whole system is protected by a radiation shield and kept in a single vacuum vessel. Since the end cap toroids are inserted into the barrel toroid system, there is an overlap in the magnetic windings. This results in a force, pulling the end cap toroids into the barrel toroid, which corresponds to about 300 tons per side. Thus, the magnet system is equipped with a force transfer system, to distribute these forces.

A central solenoid [22] completes the ATLAS magnet system. It is $5.3 \mathrm{~m}$ long and has an inner diameter of $2.4 \mathrm{~m}$, creating a $2 \mathrm{~T}$ field. The design of the solenoid required the development of new materials, since it is positioned in front of the electromagnetic calorimeter. In order to influence the passing 


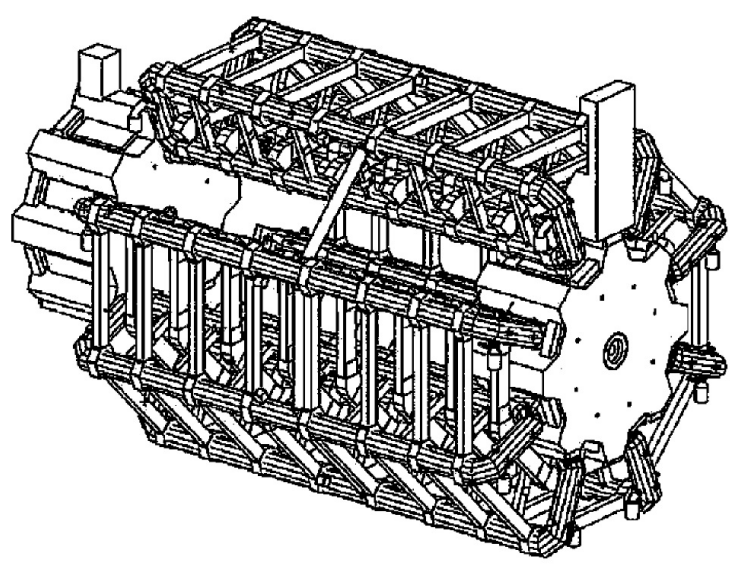

Figure 2.9: The ATLAS barrel toroid, shown in a schematic sketch. Beside the eight barrel coils, the two end cap toroids are shown, where the one on the right side is inserted to its final position and the left one extracted [21].

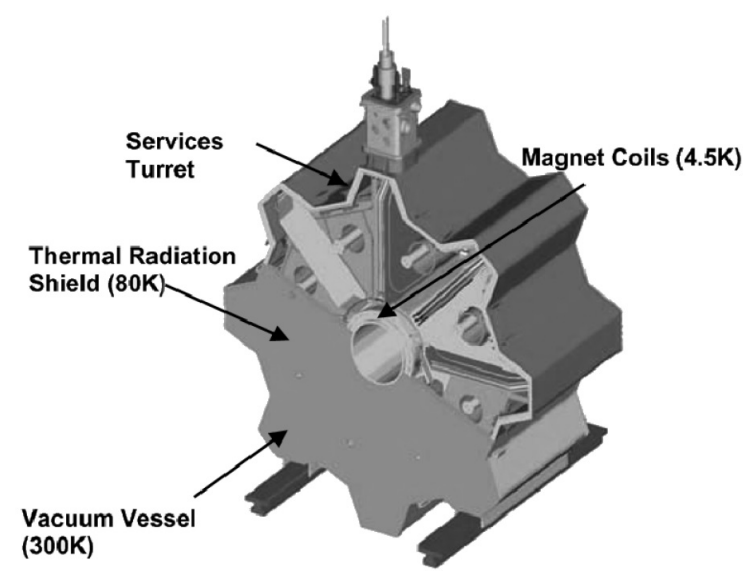

Figure 2.10: A schematic view of an ATLAS end cap toroid, showing the eight coils, which are kept in a vacuum vessel [21].

particles as little as possible, the amount of material for the solenoid had to be kept as low as possible. On the other hand, the magnet creates strong forces on the construction, which have to be absorbed by its structure. Part of the reductions in material were realized by using just a single vacuum vessel for the solenoid system and the hadronic calorimeter, instead of two separated systems.

\section{Calorimeters}

A precise knowledge of particle energies is essential for measurements focussing on discoveries, as well as measurements aiming towards higher precision in knowledge of particle properties. To measure the energies of particles, calorimeter systems are used in the ATLAS experiment. While designing a calorimeter, the main goals are to achieve good energy resolution and good linearity in the measurement performance over a large energy range. Since different kinds of particles show different interaction behaviour with material, typically the calorimeter is divided into several subsystems. In the ATLAS experiment it is realized in two different systems, the hadronic calorimeter and the electromagnetic calorimeter, as shown in figure 2.11. Both calorimeter systems are sampling detectors, providing a full $\Phi$ symmetry and coverage around the axis of the particle beam. Here sampling detector means, that the system is made of alternating layers, where one of the two layer types is typically a very dense material, used to create particle showers. The other material forms the detecting layer, measuring the width and penetration depth of the particle shower. The reason for using such a system of alternating layers is that a calorimeter made out of only sensitive material would be too large. As an example, an electromagnetic calorimeter just made out of liquid argon would have to have a thickness of around $3.5 \mathrm{~m}$ to correspond to 25 radiation lengths. In total the calorimeter systems cover a range of $|\eta|<4.9$, where $\eta$ is the pseudo rapidity, which is defined as:

$$
\eta=-\ln \left(\tan \frac{\theta}{2}\right)
$$

where $\theta$ is the polar angle, measured against the beam axis. 


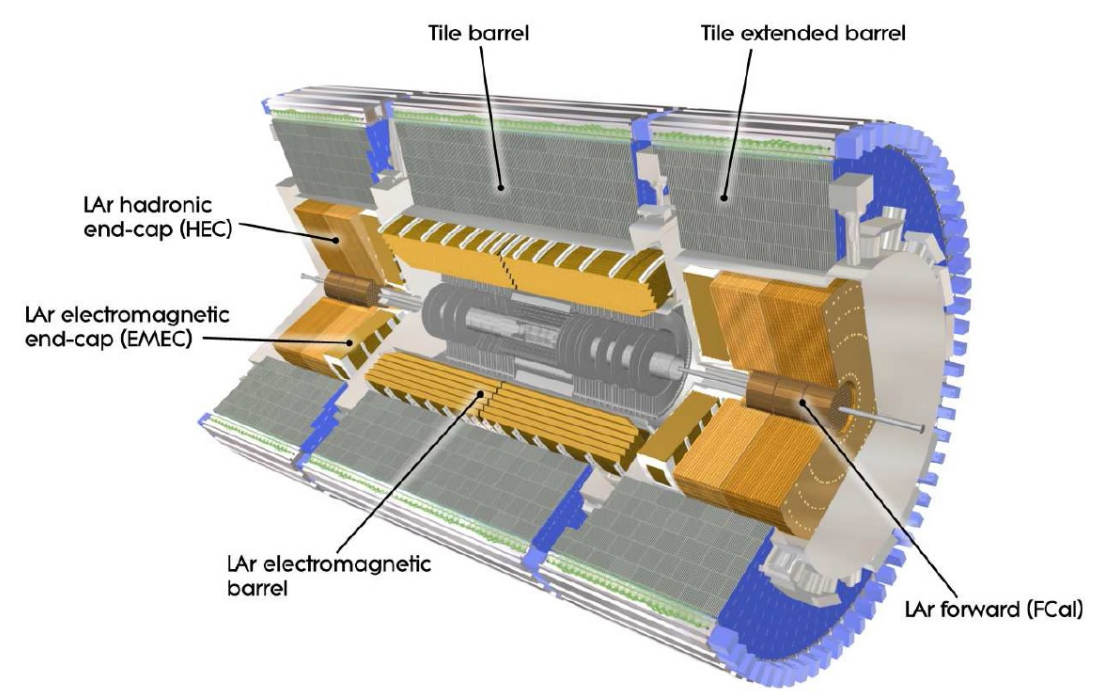

Figure 2.11: Schematic cut of the ATLAS calorimeter system, showing the different components of the hadronic and the electromagnetic calorimeter [11].

\section{Hadronic Calorimeter}

Following a typical particle track further inside the detector (towards the vertex), the hadronic calorimeters are the next part in the onion-like structure of the experiment. The hadronic calorimeter system consists of three subsystems, the tile calorimeter, the liquid argon end-cap calorimeter and the liquid argon forward calorimeter.

The tile calorimeter covers the region of $|\eta|<1.7$, which means that this part of the system is quite large. Hence steel is used as absorbing material to create the particle showers and plastic scintillator tiles as the active detecting medium, since these materials are cheaper than the alternatives.

In radial direction the tile calorimeter has a thickness that corresponds to about 7.4 interaction lengths. Figure 2.12 shows the inner structure of a tile calorimeter cutaway. The wavelength shifting readout fibres are connected to photomultiplier tubes in such a way, that the grouping of fibres corresponds to a projective geometry in terms of pseudorapidity. This ensures a homogenization of occupancies in the readout, following the definition of the pseudorapidity.

In the end-cap region between $1.5<|\eta|<3.2$, the hadronic end-cap calorimeter is mounted. It is also a sampling calorimeter system, but based on copper as absorber and liquid argon. The use of liquid argon is necessary due to its intrinsic radiation hardness. Because of its high cost and the difficult cooling, argon is only used in this region. The hadronic end-cap calorimeter again is constructed in several subsystems, whose details can be found in [11]. Two identical calorimeter end-caps are mounted on either side of the ATLAS experiment, with an outer radius of $203 \mathrm{~cm}$. Since the sensitive part of this detector is argon, it is a liquid detector. Each argon segment is equipped with three readout electrodes, at which the applied high voltage can be set for every segment individually. The design voltage for this detector is $1800 \mathrm{~V}$, which leads to a typical drift time for electrons in the liquid argon of $430 \mathrm{~ns}$.

The third part of the hadronic calorimeter is the forward calorimeter. Since the forward direction suffers from a very high radiation level, the design of the forward calorimeter brought some special challenges. To ensure a better and uniform coverage of the calorimeter, the forward part is located only $4.7 \mathrm{~m}$ from the interaction point. This short distance, compared to the size of the experiment, leads to even higher radiation, than in more conservative designs. Due to the very limited space for the forward calorimeter 


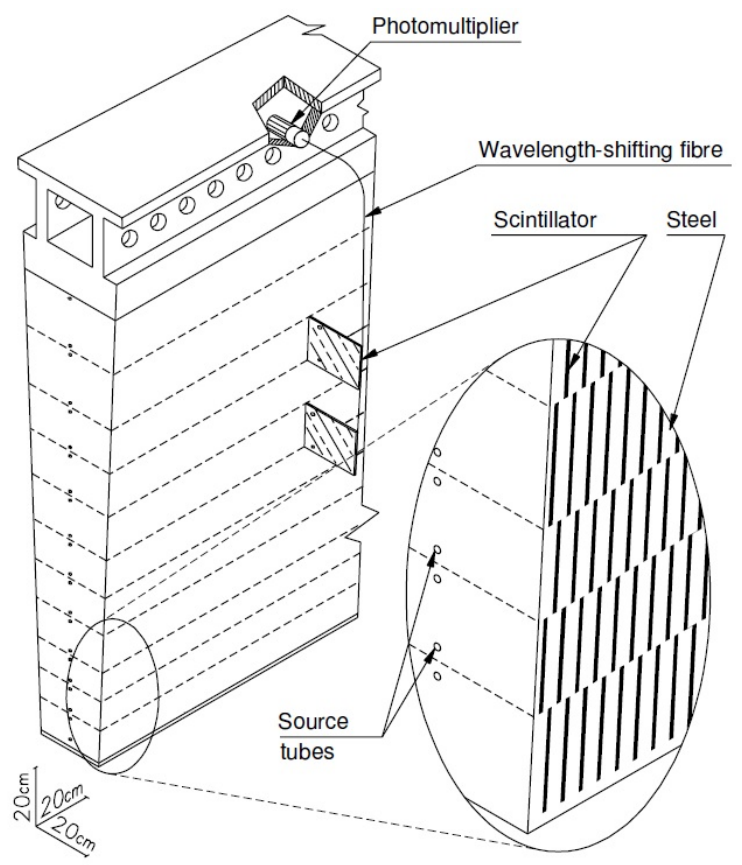

Figure 2.12: Structure of an ATLAS hadronic calorimeter cutaway view, showing the tile layout of steel absorbers and scintillators. Beside that, parts of the optical components are shown [11].

system, very dense materials had to be used, especially to realize the aimed for 9.5 interaction lengths. Beside that, a high density system avoids an energy spread to neighbouring systems, which would lead to pile-up effects in the end-cap calorimeter. To achieve the desired attributes, the forward calorimeter design is realized in three sections. Each section consists of a matrix that is made out of metal and regularly spaced rods and tubes, which are located in longitudinal channels in the metal. Here the metal matrix is grounded, while a positive high voltage is applied to the rods. The gap between the rods and the matrix is filled with liquid argon (see figure 2.13), which is the sensitive medium. In the innermost part the matrix is made out of copper, in the two other sections it is made out of tungsten. While copper has a higher sensitivity compared to tungsten and is easier in terms of handling, the stopping power of tungsten is higher due to its higher density. The combination of these two materials in the three different sections was chosen, since it fulfils the required properties in terms of interaction length and sensitivity of the forward calorimeter.

\section{Electromagnetic Calorimeter}

Covered by the hadronic calorimeter, the electromagnetic calorimeter is placed around the Inner Detector (section 2.3.2). Its purpose is the identification of electrons and photons and the measurement of their energies [23]. Beside that, the calorimeter gives the direction and position of photons, which did not interact by conversion in the tracker and is the first calorimeter that hadrons have to pass. This makes the electromagnetic calorimeter also essential for the energy reconstruction in the hadronic calorimeter. The electromagnetic calorimeter is divided into two subsystems, the barrel and the end-cap calorimeter. In contrast to the hadronic calorimeter, the subsystems of the electromagnetic calorimeter are both composed of the same materials, namely liquid argon and lead. As can be seen in figure 2.14 the calorimeter has an accordion structure. This structure ensures the hermeticity of the calorimeter, since no particle 


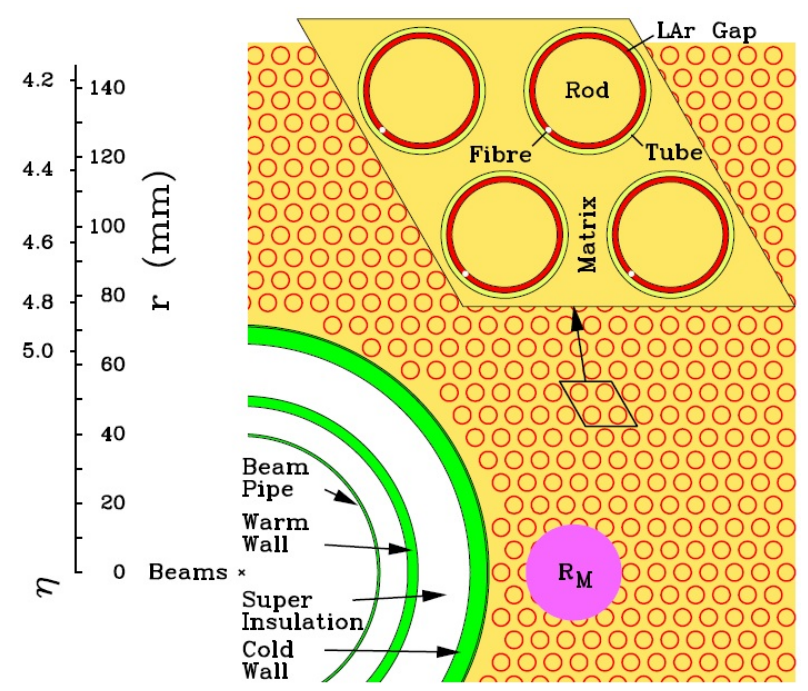

Figure 2.13: Sketch of the ATLAS hadronic forward calorimeter, showing the metal matrix structure and a zoom into the setting of the high voltage rods, which are surrounded by the active medium, the liquid argon [11].

can pass without going through the sensitive medium. Between each two consecutive absorbers, a three layer copper electrode provides the required high voltage and is also used for readout of the signals. To keep the liquid argon gap at constant thickness, the folding angles of the accordion structure vary.
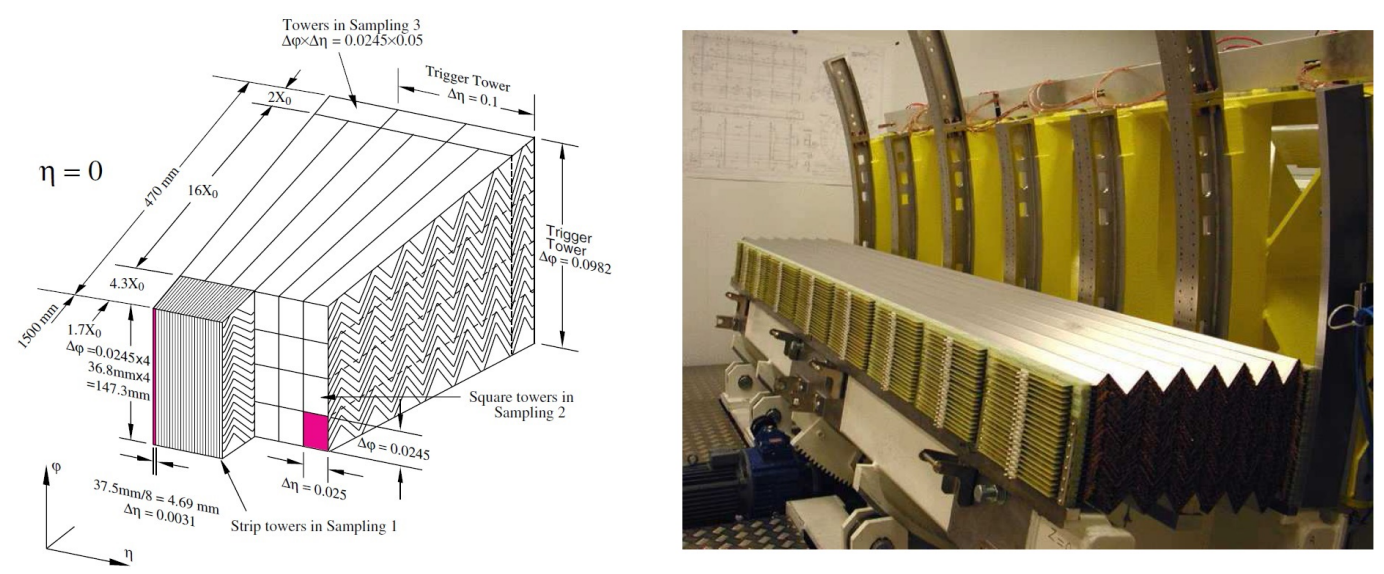

Figure 2.14: Left: Sketch of the electromagnetic barrel calorimeter at $\eta=0$. The accordion structure and the segmentation of the calorimeter is shown [23]. Right: Photograph of an electromagnetic barrel calorimeter module, which is partly stacked, and the calorimeter support rings in the back [11].

\subsubsection{Inner Detector}

The central part of the ATLAS experiment is made up of three detector systems, which are grouped into the Inner Detector. The Inner Detector is covered by the solenoid magnet. Each of the three subsystems is designed to fulfil different requirements on terms of spatial resolution, material budget and costs [24]. Stored in a cylinder of about $7 \mathrm{~m}$ length and $1.15 \mathrm{~m}$ radius, the Inner Detector is used for tracking of charged particles in a high track density environment. Tracking with high granularity around the interaction region is realized with the SemiConductor Tracker (SCT) and the Pixel Detector. To make 
the track reconstruction more robust, it is desirable to have as many tracking points as possible for each track. The Transition Radiation Tracker (TRT) gives the possibility to achieve another set of tracking points at low cost. The combination of these three technologies forms the ATLAS Inner Detector. In the following subsections an introduction to the principles of these detector systems is given.

\section{Transition Radiation Tracker}

When a highly relativistic charged particle crosses the interface between two materials with different dielectric constants, transition radiation is emitted. In the ATLAS experiment, straw tubes surrounded by foam, are used for the Transition Radiation Tracker (TRT) [25]. The foam guarantees a large number of transitions for each particle. The straw tubes contain the sensitive part of the TRT, where each tube has an inner diameter of $4 \mathrm{~mm}$. This size is a compromise, since due to the short time between two bunch crossings of $25 \mathrm{~ns}$ a short drift distance is desired, while a longer particle path through the sensitive medium gives larger signals. Each tube consists of the straw and a gaseous sensitive part. To detect the transition radiation, the straws are filled with a mixture of $70 \% \mathrm{Xe}, 27 \% \mathrm{CO}_{2}$ and $3 \% \mathrm{O}_{2}$. The transition radiation photons create a signal in the gas through the photoelectric effect. In the centre of each tube a gold plated tungsten wire is strung to read out the signals. Since the straws are kept at negative high voltage, the created electrons drift towards the readout wire. The high voltage is set to a value of around $1.5 \mathrm{kV}$, which allows operation in proportional mode.

The detector concept of the TRT provides tracking information and, in addition it allows electron identification. Since the intensity of emitted photons is inversely proportional to the mass, light particles emit more transition radiation than heavier ones. While e.g. muons only show the signature of minimum ionizing particles (see section 3.1.1) in the TRT and create relatively small signals, electrons can be identified by the larger signal due to transition radiation.

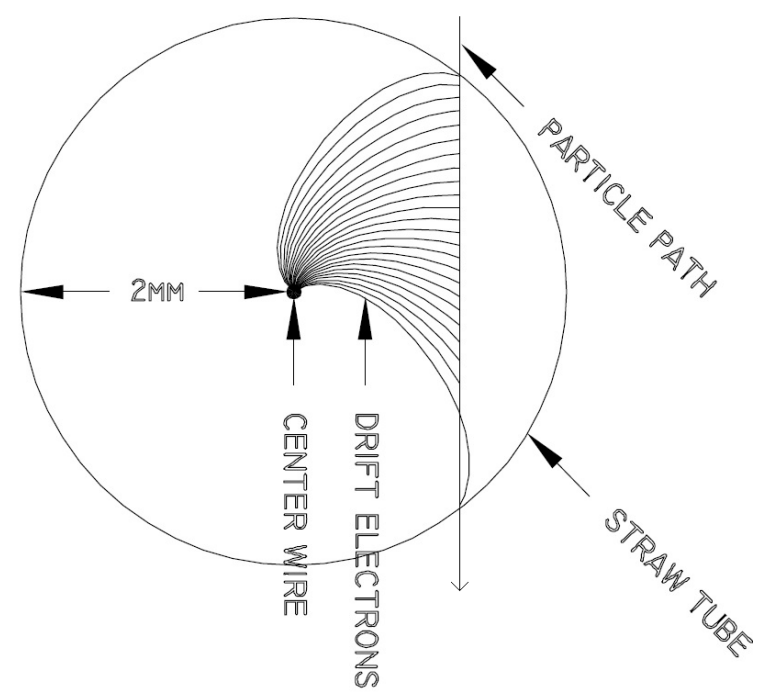

Figure 2.15: Sketch of the drift model of ionized electrons in an ATLAS transition radiation tracker straw [25].

\section{SemiConductor Tracker}

The ATLAS experiment hosts a silicon microstrip detector called SemiConductor Tracker (SCT) [26]. The SCT is made up of single detector modules organized in four cylindrical barrel layers and nine endcap discs per side, which in total adds up to an active area of $63 \mathrm{~m}^{2}$, covering the region up to $|\eta| \leq 2.5$. 
The position and orientation of the barrel and disc layers guarantee, that each particle crosses at least four layers of the SCT.

Each SCT module consists of two $285 \mu \mathrm{m}$ thick planar silicon p-in-n strip sensors, which are glued back-to-back under a stereo angle of $40 \mathrm{mrad}$. Resulting in two-dimensional space points for every hit, this setup allows to resolve hit ambiguities, since only one crossing point of each two sensor strips is within the sensor volume. The length of the used strips is about $12 \mathrm{~cm}$ with a strip pitch of about $80 \mu \mathrm{m}$, depending on the position of the module (barrel/disc). More than 4000 modules of this kind are used. Due to the orientation of the modules, the spatial resolution provided in $R \phi$ direction is $16 \mu \mathrm{m}$ and in $\mathrm{z}$ direction (along the beam axis) $580 \mu \mathrm{m}$. For operation the whole SCT is cooled down to $-7^{\circ} \mathrm{C}$ to avoid reverse annealing effects. When a charged particle passes the silicon detector, it creates charge carriers along its track, which induce charges on the strips. These signals are read out by binary frontend electronics, which feed the information to optical components. The information is transformed on the on-detector side to light pulses and sent to the counting rooms. A photograph of an SCT module is shown in figure 2.16, highlighting all components of this exemplary module. The less complicated structure of SCT modules and read-out electronics together with easier production methods, compared to ATLAS pixel modules (described in section 3), were among their main advantages in direct comparison. Combined with the good achievable spatial resolution and the lower production costs, the silicon strip detector technology was chosen as outer part of the ATLAS silicon tracker system.

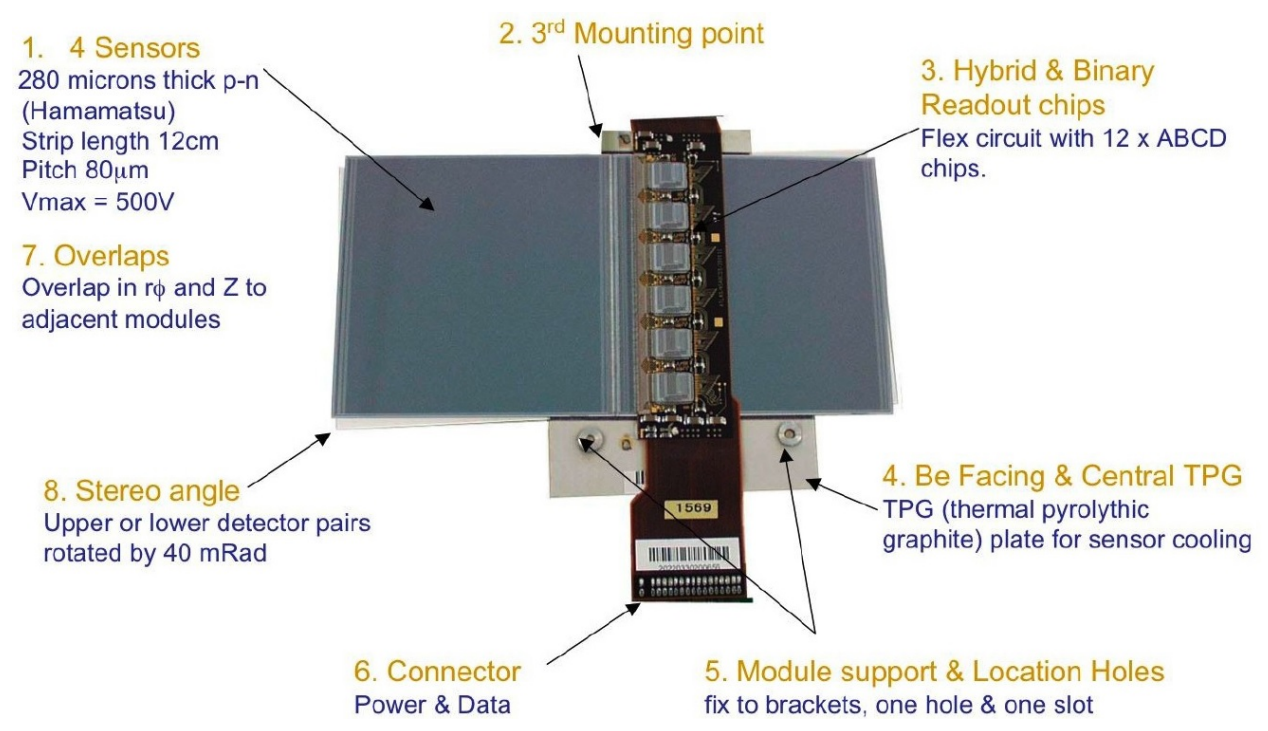

Figure 2.16: Photograph of an ATLAS SemiConductor Tracker end-cap module, indicating the different components of the module [26].

\section{Pixel Detector}

The innermost detector system of the ATLAS experiment is the silicon pixel detector. A detailed description of the current pixel detector, as well as ongoing and planned upgrade perspectives are given in chapters 3 and 4 . 


\section{Chapter 3}

\section{The ATLAS Pixel Detector}

The pixel detector is the innermost sub-detector of the ATLAS experiment, used for high precision tracking at high particle rates. In the following sections an overview of the most important effects for tracking devices based on silicon is given, followed by a detailed description of the ATLAS pixel detector itself.

\subsection{Silicon Detector Basics}

When designing a detector for high energy physics experiments, like the ATLAS experiment, the demand for the systems closest to the interaction point is to provide excellent tracking capabilities, combined with a fast readout and low material budget. These requirements towards the inner detector design are driven by the need for correct vertex reconstruction and low multipile scattering. The best compromise between these requirements are solid state detectors, providing a large signal per crossed particle, which allows for precise tracking. Good high voltage stability and the possibility to thin down solid state materials while still being usable, ensures a fast readout and only a small fraction of a radiation length as material budget.

\subsubsection{Charged Particles in Matter}

When a heavy charged particle crosses matter, it mostly looses energy through elastic collisions with the atoms' electrons, which leads to excitation and ionization of the atoms [27, 28]. In this process, free charge carriers are created, which form a signal that can be read out. The average energy loss of an incident charged particle, and thus also the number of created charge carriers, can be calculated using the Bethe-Bloch formula:

$$
-\left\langle\frac{d E}{d x}\right\rangle=2 \pi N_{a} r_{e}^{2} m_{e} c^{2} \rho \frac{Z}{A} \frac{z^{2}}{\beta^{2}} \cdot\left[\ln \left(\frac{2 m_{e} \gamma^{2} v^{2} W_{\max }}{I^{2}}\right)-2 \beta^{2}-\delta-2 \frac{C}{Z}\right]
$$

with (in case of silicon): 


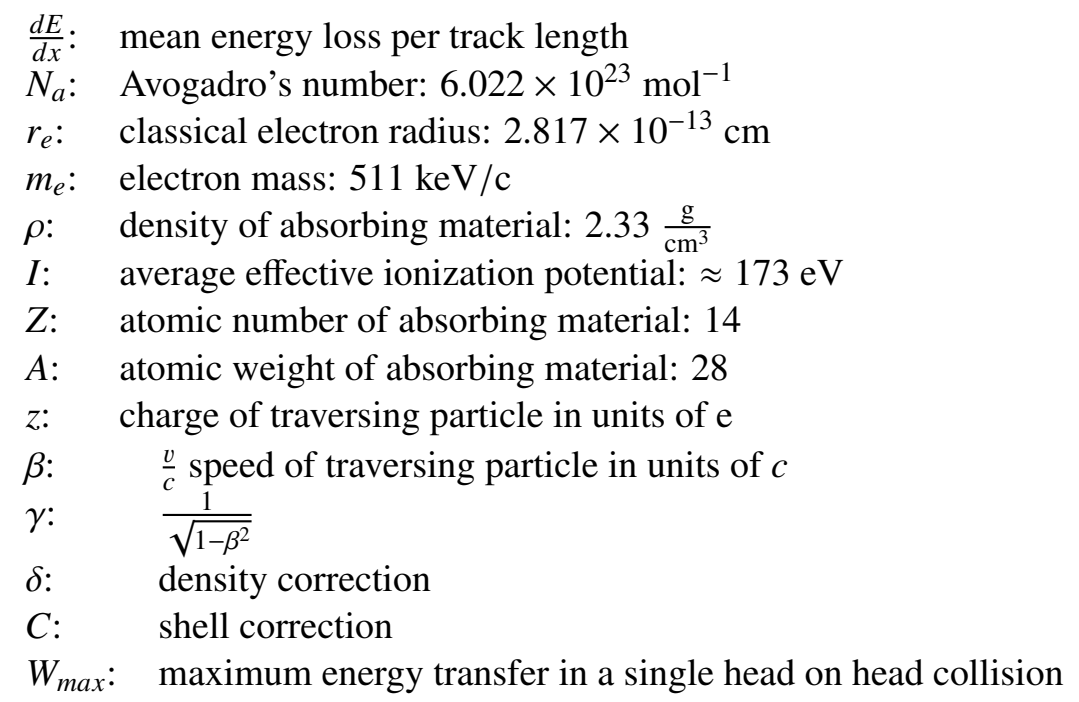

The average stopping power of a muon versus $\beta \gamma$ is shown in figure 3.1 for copper as the penetrated material.

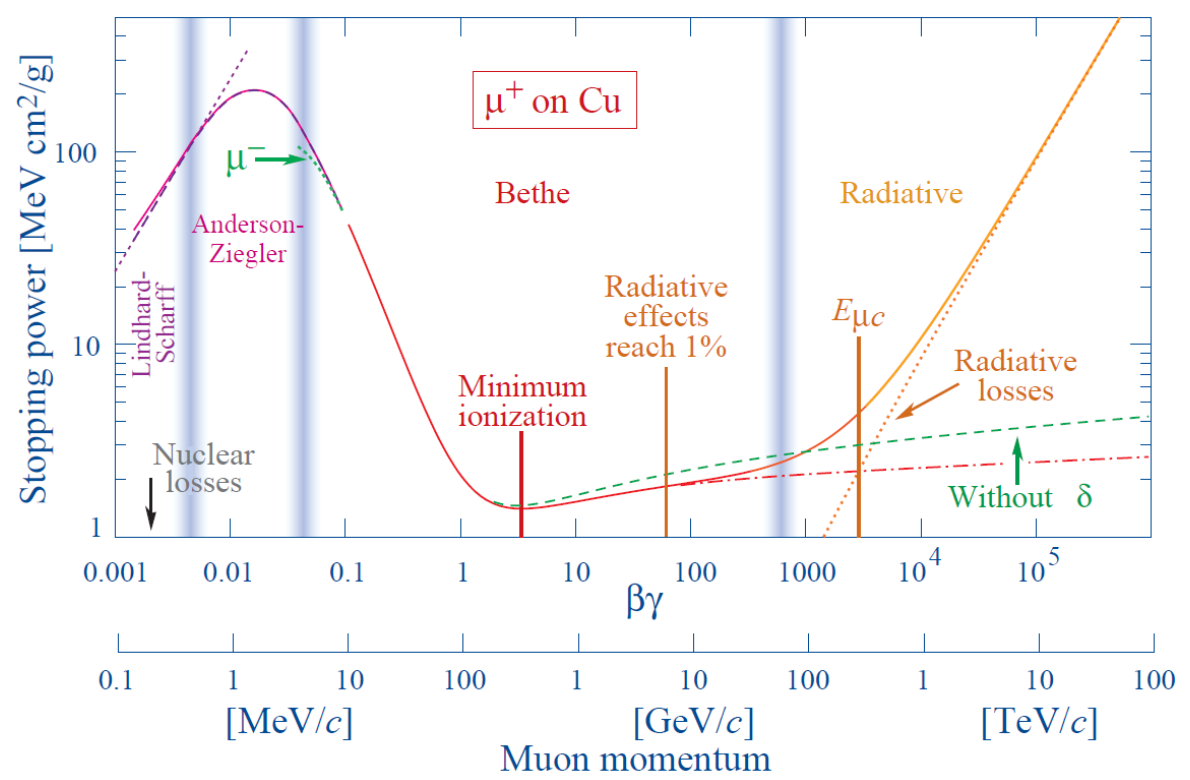

Figure 3.1: Average stopping power of muons in copper for different ranges of $\beta \gamma$. The light blue vertical bands indicate the limits of different models, used as approximations [29].

As can be seen in figure 3.1, nuclear losses are the dominating effect for low particle momenta and bremsstrahlung dominates for high momenta. The average stopping power has a minimum at around $\beta \gamma \approx 3-4$. Incident particles with kinetic energies around that minimum are called "minimum ionizing particles" (mip), which, consequently, create the smallest signals in the sensor. For typical silicon sensor thicknesses of few $100 \mu \mathrm{m}$ in high energy physics experiments, a mip creates on the order of 20,000 electron/hole pairs. When going to higher values of $\beta \gamma$, the increase in stopping power is logarithmic. Consequently, the deviations for high particle momenta are just on the order of $10 \%$, compared to the expected value for a mip. Thus, a typical value, that can be used as a guideline for a large variety of materials, is $\left\langle\frac{d E}{d x}\right\rangle_{\text {mip }} \approx 1.5 \mathrm{MeV} \frac{\mathrm{cm}^{2}}{\mathrm{~g}}$. 
The Bethe-Bloch formula gives the average energy loss for different incident particle momenta. The real values for the energy loss of a bunch of incident particles are distributed around this average value. For the case of a thin material layer, as it is typical for high energy physics detectors, only few interactions between an incident particle and the detector material will happen. Here the probability for interactions with small energy transfers is much higher, than the probability for the maximum possible energy transfer, which requires a head-on collision. Hence, the shape of the distribution is very asymmetric for thin detector materials and follows a Landau distribution, as shown in figure 3.1. In the testbeam measurements, described in chapter 7 , the expected shape of a charge spectrum can be described by this model.

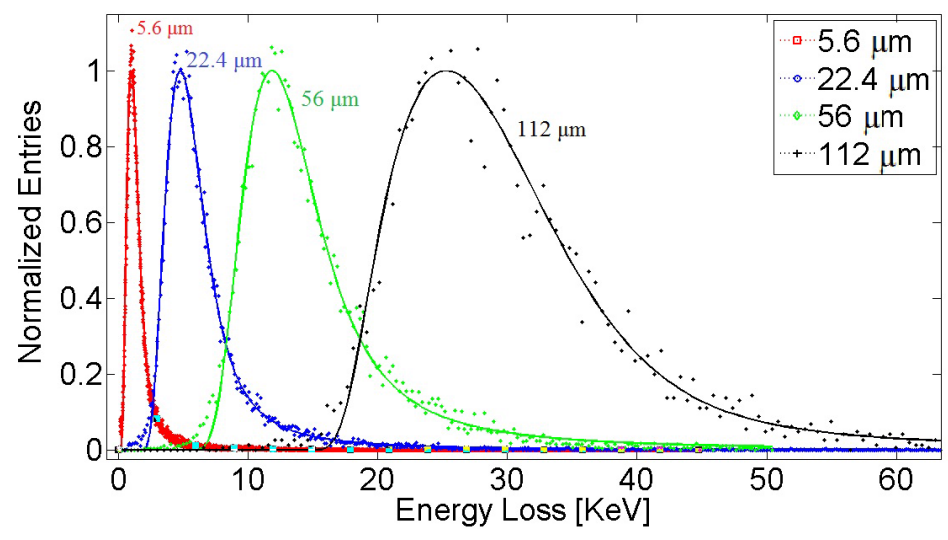

Figure 3.2: Energy loss for $12 \mathrm{GeV}$ protons in silicon absorbers of different thicknesses. Landau functions are fitted to the different distributions [30].

\subsubsection{Energy Loss of Photons in Matter}

The interaction of photons with matter mainly happens through the photoelectric effect, Compton scattering and pair production. One of the main differences between photons and charged particles in the interaction with matter is that charged particles loose energy while traversing material, while photons are absorbed through two of the three mentioned processes. Thus, the energy of an incident photon beam does not change very much, but the intensity does. Here the drop in intensity follows an exponential law, described by $I(x)=I_{0} \cdot e^{-\mu x}$, where $I_{0}$ is the photon intensity before interaction with the material, $\mu$ is the absorption coefficient of the material and $x$ the depth. Depending on the energy that is transferred from a photon to an electron, the electron can either be excited to the conduction band or to intermediate states, see figure 3.3.

For photon energies of few $\mathrm{keV}$, which are typical energies for detector lab tests using radioactive sources or lasers, the photoelectric effect is dominant (see figure 3.4). Here a photon is absorbed by an atomic electron of the used material and this electron is ejected from the atomic bond. The cross section for this process decreases rapidly with photon energy, with the exception of the values that correspond to the L- and K-shell energies. For slightly higher energies, on the order of MeV, Compton scattering, the scattering of photons at the (quasi-)free electrons, is the dominant process, which ionizes the atom. For higher energies, pair production, the creation of an electron-positron pair, becomes the dominant process, where the minimum threshold energy for the process is $1.022 \mathrm{MeV}$. 

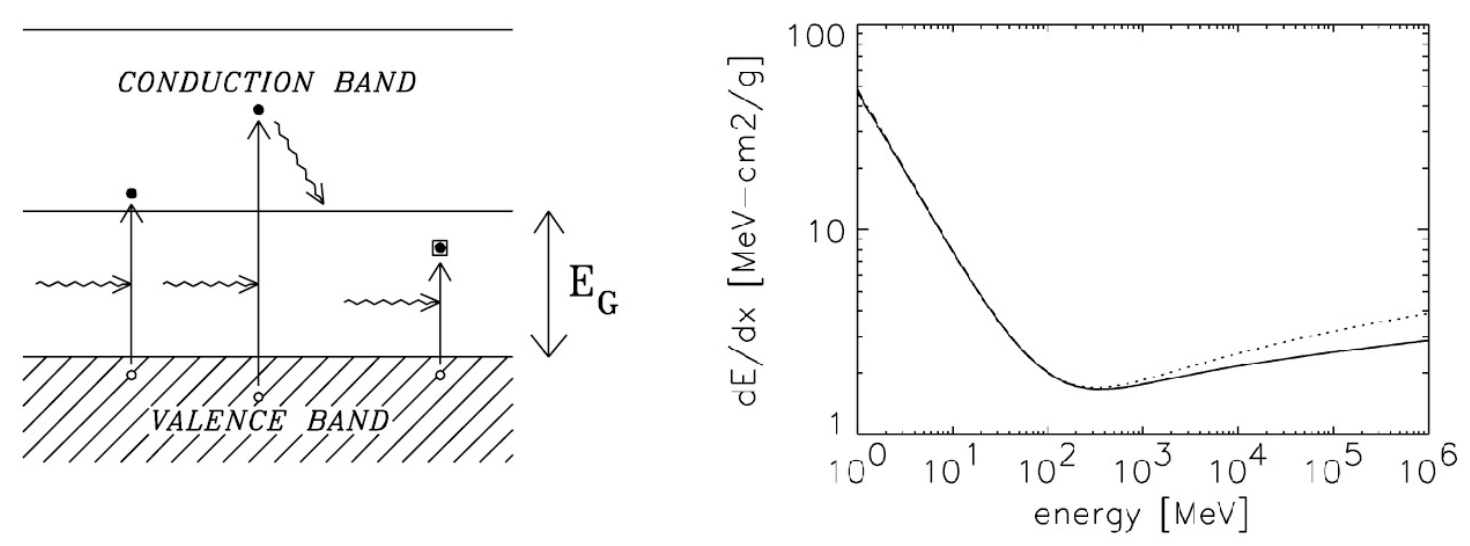

Figure 3.3: Left: Photons with energies equal, larger and smaller than the band gap are absorbed and lift electrons to an intermediate state or the conduction band. Simultaneously holes are created in the valence band. Right: Energy loss of a charged pion in silicon due to ionization versus the kinetic energy of the pion. The dotted line shows the progression including shell and density corrections [28].
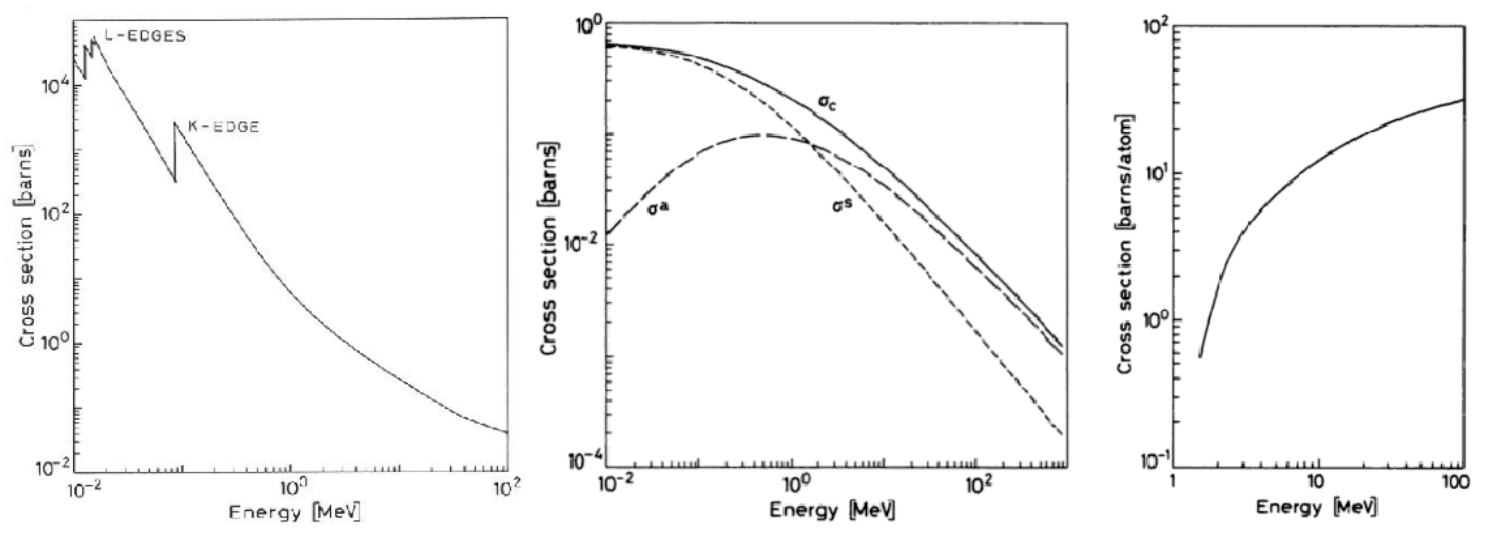

Figure 3.4: Left: cross section vs photon energy for the photoelectric effect (in lead), centre: cross section vs photon energy for Compton scattering and right: cross section vs photon energy for pair production [27].

\subsubsection{Energy Bands}

Solid state materials can roughly be categorized in three groups, insulators, conductors and semiconductors. They are distinguished by the energy bands that electrons fill. For insulators, the valence band, in which holes are mobile, and the conduction band, in which electrons are mobile, are separated in terms of energy by a large band gap (on the order of $10 \mathrm{eV}$ or more). Conductors either have an overlapping band structure or a partially filled conduction band, which results in a high mobility of electrons in the material, meaning that they are highly conductive. The third material group is somewhere in between them, with a band gap of few $\mathrm{eV}$, resulting in very interesting properties. A schematic drawing is shown in figure 3.5 .

The population of the conduction band with electrons is temperature dependant. In the case of semiconductors, the thermal energy is sufficient to continuously create electron/hole pairs. Although, in most cases the band gap is too large for direct thermal excitation, electrons can be excited to intermediate states created by the impurities in the material, and in a second step excited to the conduction band. In 


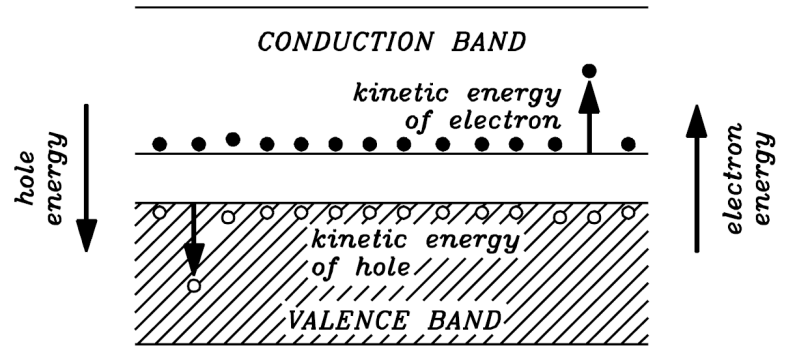

Figure 3.5: Schematic drawing of the energy band structure for semiconductors, with a band gap on the order or few eV [28].

case of silicon, the probability of direct thermal excitation smaller than the band gap of $1.2 \mathrm{eV}$ would suggest, since silicon is an indirect semiconductor and additional energy is necessary to bring electrons to the conduction band. The required energy to create an electron/hole pair in silicon is $3.6 \mathrm{eV}$, where the energy difference of $2.4 \mathrm{eV}$ is necessary for the creation of phonons.

In general, the concentration $n_{i}$ of electrons (and holes) at a fixed temperature is given by:

$$
n_{i} \sim T^{3 / 2} \exp \left(\frac{-E_{g}}{2 k T}\right)
$$

where $T$ is the temperature, $E_{g}$ the band gap of the material at $0 \mathrm{~K}$ and $k$ the Boltzmann constant. At room temperature a typical value of thermal charge carriers in silicon is $1.5 \cdot 10^{10} \mathrm{~cm}^{-3}$. Compared to the thermal charge carrier concentration, the number of electron/hole pairs created by a mip is extremely small.

\subsubsection{Doping of Semiconductors}

When using a semiconducting material for particle detection, the material can be categorized to be either an intrinsic or extrinsic semiconductor. Intrinsic semiconductors are contaminated with just very few (ideally no) impurities, compared to the number of electrons (and holes) that are thermally generated. In reality it is not only extremely difficult to produce pure semiconductors, but other materials are even added on purpose to create specific kinds of impurities. Either during the growth process of the crystal or later just in selected regions of the material, small fractions of another material are added to produce an extrinsic semiconductor with specific properties. This process is called doping. Depending on the type of impurity that is added, the material is called n-type (which means a material with an excess of electrons in the conduction band) or p-type (which describes a material with additional holes in the valence band), shown in figure 3.6.

\subsubsection{Silicon as detector: $p-n-j u n c t i o n$}

When a particle crosses silicon and deposits energy through one of the processes mentioned above, on average for each deposited $3.6 \mathrm{eV}$ an electron-hole pair is created. For the example of a minimum ionizing particle, crossing a silicon detector of $200 \mu \mathrm{m}$ thickness, on average $\frac{\langle\Delta \mathrm{E}\rangle}{W_{s i}} \approx 21600$ electronhole pairs are created. This charge signal in principle is large enough to be processed by appropriate readout electronics. 

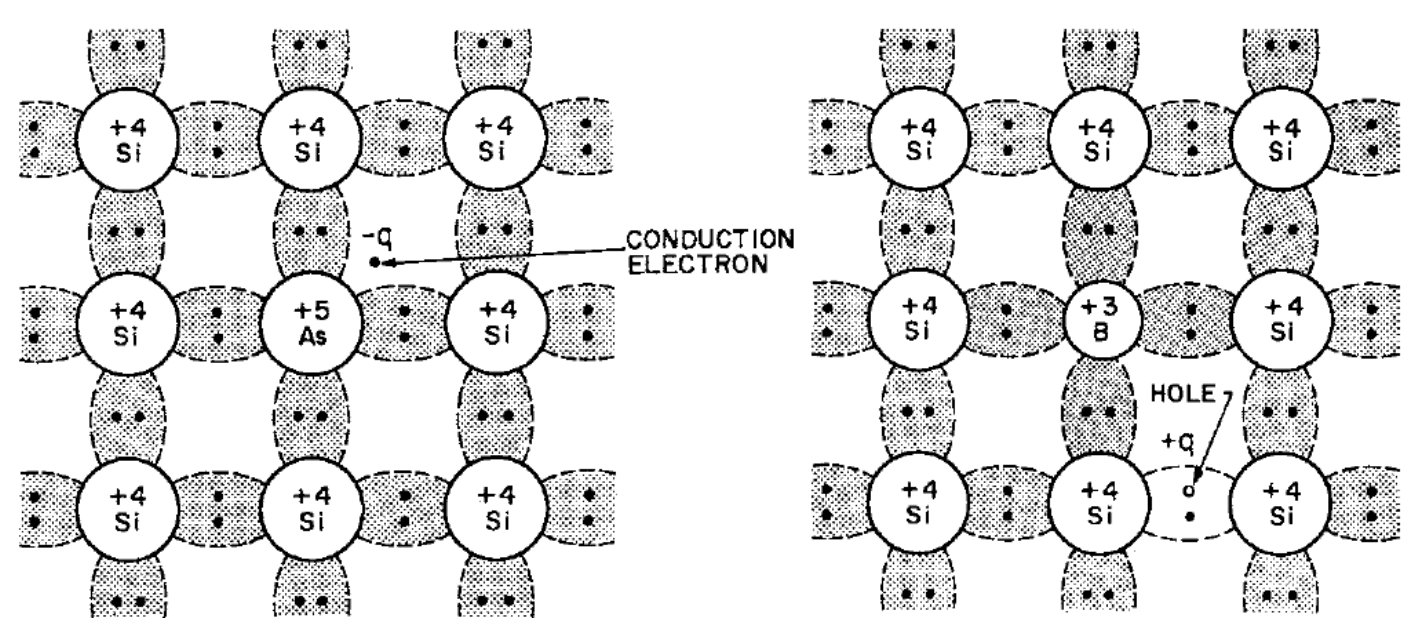

Figure 3.6: Examples for doped silicon: n-type semiconductor with an excess of electrons (left), p-type semiconductor with an additional holes (right) [28].

In order to be able to detect such a signal, the sensitive layer needs to be depleted, which means that ideally no free charge carriers should be present. To be able to create a depletion zone, a p-n junction in the material is required. This can be realized by merging one p-doped part of e.g. silicon and one n-doped part of silicon. Due to a diffusion process of the charge carriers, electrons and holes annihilate around the contact region of the two doped material fractions. The diffusion process results in an electrical field, pointing against the direction of motion of the charge carriers, such that an equilibrium is reached and the contact region stays depleted. By applying an external voltage in reverse bias mode, the potential drop between the n-doped and p-doped region can be increased, which leads to an expansion of the depletion zone. For proper detector operation, the voltage is set to a value, which is sufficient to expand the depletion zone throughout the whole sensor thickness. In a fully depleted sensor, the complete signal created by an incident particle, reaches the readout electrodes and can be detected. The required depletion voltage $V_{d e p l}$ is proportional to the doping concentration $N_{D}$ :

$$
V_{d e p l}=\frac{q N_{D}}{\epsilon \epsilon_{0}} \frac{d_{D e t}^{2}}{2}-V_{b i}
$$

where $d_{D e t}$ is the detector thickness and $V_{b i}$ the built-in voltage, developed by the p-n junction. Thus, external voltages on the order of several $10 \mathrm{~V}$ are applied to unirradiated silicon detectors during operation.

In detector operation, the signal generation process that is caused by a drift of the generated charge carriers in the depletion region, can be described by the Shockley-Ramo theorem [31]. The theorem describes the induced charge $Q$ on a readout electrode and the corresponding current in the readout device $I:$

$$
\begin{aligned}
& Q=-q \cdot \varphi_{0}(\mathbf{x}) \\
& I=q \cdot \mathbf{v} \cdot \mathbf{E}_{0}(\mathbf{x})
\end{aligned}
$$

Here $q$ is the moving point charge, $\mathbf{v}$ its instantaneous velocity, $\varphi_{0}(\mathbf{x})$ the weighting potential at $q$ 's current position and $\mathbf{E}_{0}(\mathbf{x})$ the weighting field. 


\subsubsection{Multiple Scattering}

Beside the ionization and excitation processes described by the Bethe-Bloch formula, incident particles are scattered at the detector material's nuclei. The angular distribution of scattering processes themselves can be described by the Rutherford formula, while the basic work on this topic has been done by Molière [32]. The basic outcome is, that the cross section for small scattering angles is large. This means that the total scattering of a particle, when crossing a layer of material of thickness $d$, is a consequence of a large number of scatter processes with small angles. When histogramming the total scattering angle $\theta$, the distribution can be fitted by a Gaussian, which is centred around zero and has a standard deviation empirically described by:

$$
\sigma_{\theta} \approx \frac{13.6 \mathrm{MeV}}{p \cdot \beta} \sqrt{\frac{d}{X_{0}}}
$$

with $p$ as the momentum of the incident particle, $\beta$ the relativistic velocity of the particle and $X_{0}$ the radiation length, which is the mean distance of high energetic electrons in a material to loose $1 / \mathrm{e}$ of their energy. Multiple scattering effects limit the tracking precision in measurements, especially for low momenta. This plays an important role for testbeam measurements, where the tracking precision increases with higher beam momentum, lower material thickness and higher radiation length of the material. Especially the first two parameters usually can be changed by the users.

\subsubsection{Radiation Damage in Silicon Detectors}

Due to the position closest to the interaction point, the ATLAS pixel detector suffers most from radiation damages. Different types of radiation damages occur in the detector modules. To investigate the radiation effects on the detector performance during the lifetime of the experiment, testbeam measurements with highly irradiated devices are performed. The main processes of radiation damage, which occur in silicon, are briefly discussed in the following [33]. Since the performance limiting radiation damages mostly occur in the sensor bulk, surface defects are not treated.

Atoms can be displaced out of the crystal structure, if e.g. an incident hadron or high energetic lepton transfers a significant part of its energy via Coulomb interaction to the silicon atom. As result, the atom is removed from the lattice and a vacancy is left over. The often quoted recoil energy, that is necessary for this process was simulated to be around $25 \mathrm{eV}$ [34]. At this energy the probability to displace an atom is around $50 \%$ (for silicon). In case the recoil energy stays below this value, most probably the deposited energy leads to lattice vibrations in the crystal. For values above $25 \mathrm{eV}$ most probably a pair of a displaced atom and the corresponding vacancy is created. For much higher energies not only a displacement-vacancy pair can be created, but the displaced atom can have enough energy to perform the same processes again.

The total amount of radiation damage in a material does not only depend on the energy of the incident particles, but also on the particle type. While charged particles mainly interact through Coulomb scattering and only partly with the nucleus of the atom, neutral particles interact with the nucleus only. Beside that, the mass of the incident particle has an effect on the transferred energy. Table 3.1 gives an overview on the damage characteristics of different incident particles, hitting a silicon layer.

The table clearly shows, that the damage potential and characteristics differs, depending on the incident radiation. To quantify the radiation dose accumulated by a running experiment or gained in an irradiation campaign, a common scale was introduced, which specifies the dose in $1 \mathrm{MeV}$ neutron equivalents. The theory behind this scaling is the non-ionizing energy loss (NIEL) hypothesis. As briefly discussed before, the damage of a crystal lattice depends on the particle properties. Assuming, that the 
particle fluence $\Phi(E)$ is known, a damage parameter $K$, which is normalized to $1 \mathrm{MeV}$ neutrons, can be defined. The damage parameter depends on the damage function $D(E)$ through:

$$
K=\frac{\int D(E) \cdot \Phi(E) d E}{D_{n}(1 \mathrm{MeV}) \int \Phi(E) d E}
$$

The calculated damage parameters e.g. for the current innermost ATLAS pixel layer are $2 \cdot 10^{15} \frac{\mathrm{n}_{\mathrm{eq}}}{\mathrm{cm}^{2}}$ and for the Insertable b-Layer (section 4) after Run 2 the damage parameter is $5 \cdot 10^{15} \frac{\mathrm{n}_{\mathrm{eq}}}{\mathrm{cm}^{2}}$.

\subsection{Current ATLAS Pixel Detector Layout}

The central detector system of the ATLAS experiment is the pixel detector. It consists of three barrel layers and three end-cap discs per side. The barrel layers are mounted very close to the beampipe, at radii of $122.5 \mathrm{~mm}$ (the outermost "layer 2"), $88.5 \mathrm{~mm}$ ("layer 1") and $50.5 \mathrm{~mm}$ ("layer 0", also called "b-layer") being the component closest to the beampipe. An overview of the ATLAS pixel system is shown in figure 3.7. In total the pixel detector has a length of around $1.4 \mathrm{~m}$, where the arrangement of the barrels and discs guarantees that each particle hits at least three layers up to $|\eta|<2.5$. Some of the main attributes of the pixel detector are the high granularity and fast readout time. Both are necessary to fulfil the task of vertex detection and especially to separate different vertices occurring during the same bunch crossing. For a long-lived particle, like a bottom quark, the particle can move a few millimetres away from the initial vertex. Due to the requirement of being able to distinguish the two (or more) vertices accurately, the pixel detector needs a very high spatial resolution, which corresponds to choosing small pixel sizes. Each (standard) pixel of the current modules has a dimension of $400 \times 50 \mu \mathrm{m}^{2}$. This results in a total number of about 80 million pixels in total. For the ATLAS pixel system the measured resolution in $\mathrm{R} \Phi$ direction is $12 \mu \mathrm{m}$. To reach this value, charge sharing effects between the pixels are taken into account to improve the resolution.

In order to minimize effects on the particles' trajectories, the amount of material had to be kept as low as possible. This was achieved by choosing materials with a long radiation length and by thinning down the components to their minimal technical requirements. As result, the whole pixel barrel has $10.7 \% X_{0}$ for $\eta=0$, where $7.2 \% X_{0}$ is caused by support structures and cooling and the remaining $3.5 \% X_{0}$ comes from the pixel module itself.

\subsubsection{Module Layout}

Figure 3.8 shows the hybrid structure of a pixel barrel module. Each of these modules consists of a $250 \mu \mathrm{m}$ thick $\mathrm{n}^{+}$-in-n silicon sensor, covering an area of about $2 \times 6 \mathrm{~cm}^{2}$. To provide a readout circuit for each of the 47232 pixel cells per sensor, 16 Front-End (FE) chips of the I3 generation are bumpbonded to the sensor. To organize the distribution of trigger and command signals for all $16 \mathrm{FE}$ chips, as well as to combine the outgoing data streams, each module flex is equipped with a module control chip (MCC). The MCC sets up the communication channel to optical components, which provide the connection to counting rooms. Both, the FE chips and the MCCs, are thinned down to less than $200 \mu \mathrm{m}$, to keep the material budget low. A short overview of the current pixel sensor and the FE-I3 chip is given in the following two subsections, especially for comparison to new designs in section 4.1. 


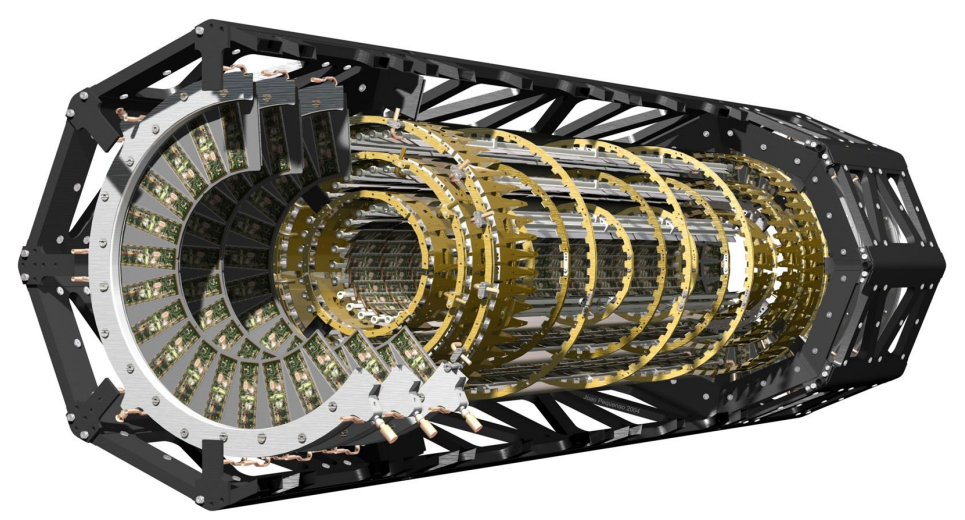

Figure 3.7: The ATLAS pixel detector system, showing the three cylindrical barrel layers, as well as three end-cap discs per side (ATLAS Experiment (C) 2013 CERN).

\subsubsection{The Sensor}

The current ATLAS pixel sensors are made of $250 \mu \mathrm{m}$ float-zone silicon, which is n-doped [35]. Measurements during the design phase showed that an oxygen enrichment of silicon increases the radiation hardness, which comes with a lower depletion voltage over time, compared to standard silicon (figure 3.9). With increasing irradiation of the sensor, the number of acceptor-like donor states in the energy gap increases, which leads to a decreasing concentration. After a certain irradiation level, the initially n-type material behaves like a p-type material ("type inversion"). With further increasing irradiation levels, the effective doping concentration and consequently also the depletion voltage rises to very high values. The oxygen enrichment dampens this effect and thus keeps the required depletion voltage lower. For this reason, also the ATLAS IBL sensors are made of diffusion oxygenated float-zone (DOFZ) silicon.

As previously mentioned, the standard pixel size in the sensor design of the current ATLAS pixel detector is $400 \times 50 \mu \mathrm{m}^{2}$, which are the dimensions for 41984 pixels per sensor. The remaining 5248 pixels of each sensor have a size of $600 \times 50 \mathrm{~m}^{2}$ (called "long pixels"), which is necessary to cover the $200 \mu \mathrm{m}$ gaps between each two FE chips. Further special pixel designs ("ganged pixels") are necessary in the outer rows of the sensor.

For n-in-n design sensors, the side opposite to the pixel implants needs to be p-doped to create a junction. The sensor edges are segmented into 17 circumferential rings, which are used to bring down the high voltage potential from the main implant to the edges in a stepwise way. This is necessary to avoid high voltage sparks around the cutting edge of the sensor, which could destroy the FE chip. The pixel implants are of $\mathrm{n}^{+}$type, which means highly $\mathrm{n}$-doped, while the sensor bulk is of normal $\mathrm{n}$-type silicon. This allows a partial depletion from the implant side after type inversion, which permits a partially depleted sensor operation after this point, as shown in figure 3.11.

Another important detail in the sensor design are extra $\mathrm{n}^{+}$implants in each pixel cell. These implants are connected to a metallization grid, which is used to apply a potential to the pixel cells for test reasons. These high voltage tests were necessary to qualify sensors without having a FE chip connected, which is an essential way of cost efficient sensor testing. A disadvantage of these extra high voltage pads ("bias dots") is a charge loss, which results also in an efficiency drop, around that region in the cells, as shown in section 7.4. Since the possibility to test sensors without FE chip is so important in sensor qualification, also the later described IBL sensor designs include a bias dot. 


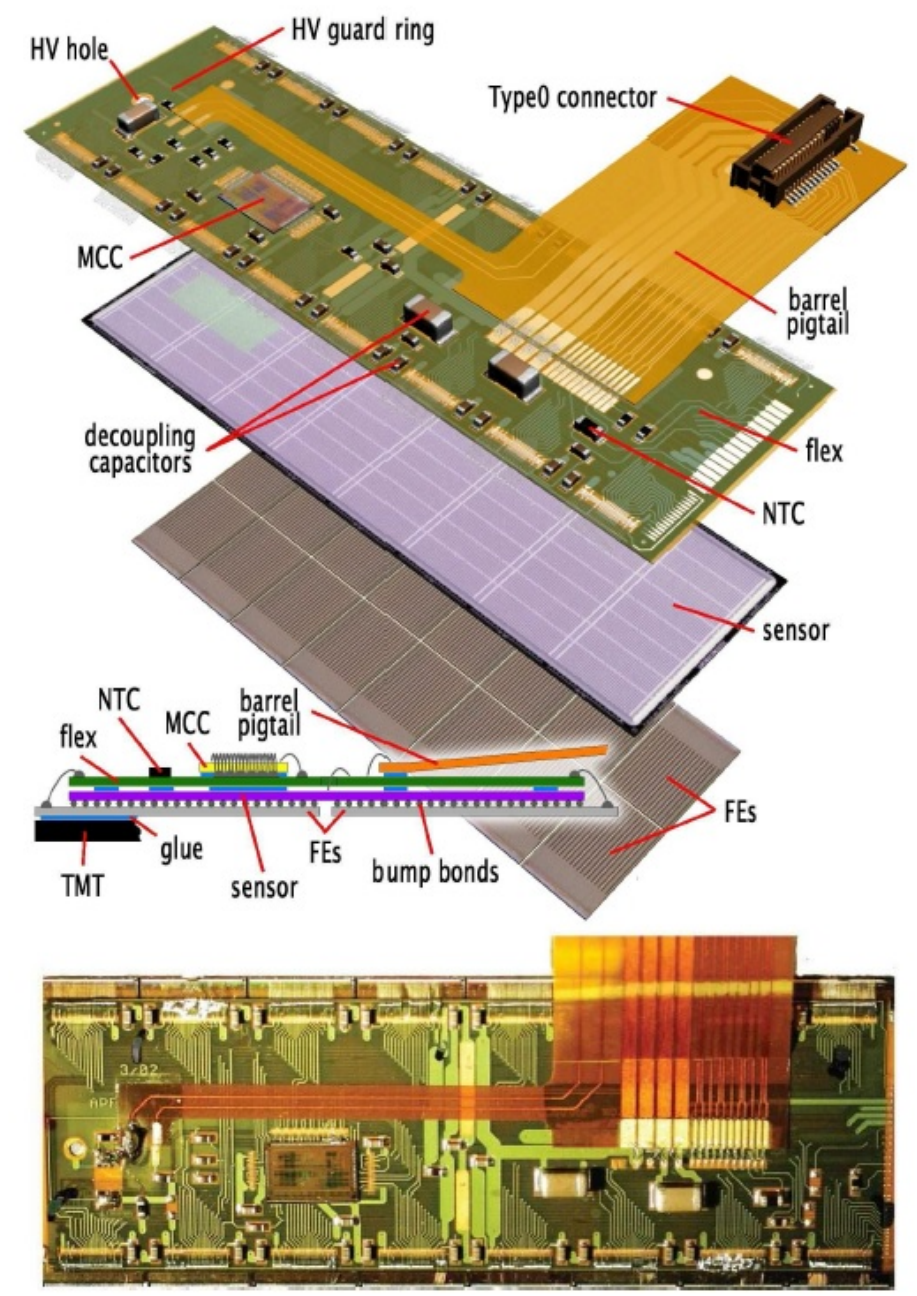

Figure 3.8: Top: Schematic drawing of a barrel ATLAS pixel module, illustrating the hybrid module concept. Shown are the module flex, including electronics to handle the different Front-End chips, the sensor itself and the 16 Front-End chips. Bottom: Photograph of a full pixel module [11].

\subsubsection{The Front-End Chip}

The Front-End chip is the readout part of a pixel module [38]. It is the first electronics part in processing signals, transforming them to digital data and the last part in the command receiving chain of the ATLAS pixel system. The chip was produced in a $250 \mathrm{~nm}$ CMOS process to increase the radiation tolerance. Each FE chip cell has an analogue and a digital part. In the analogue part, which is shown in figure 3.12, the signal either comes from the sensor or, for test reasons, from an injection capacitance. Subsequently, a preamplifier amplifies the signal and charges the feedback capacitor, which is discharged by a constant current. The discharge current can be set individually for each cell. This ensures an approximately linear relation between the detected charge and the time the signal is above the threshold (time over threshold, ToT) of the next electronics part in the chain, the discriminator. Thus, the discriminator output gives a direct measure of the signal amplitude, as shown in figure 3.13.

To ensure a uniform response of all pixel cells for a given injected charge, the discriminator and 


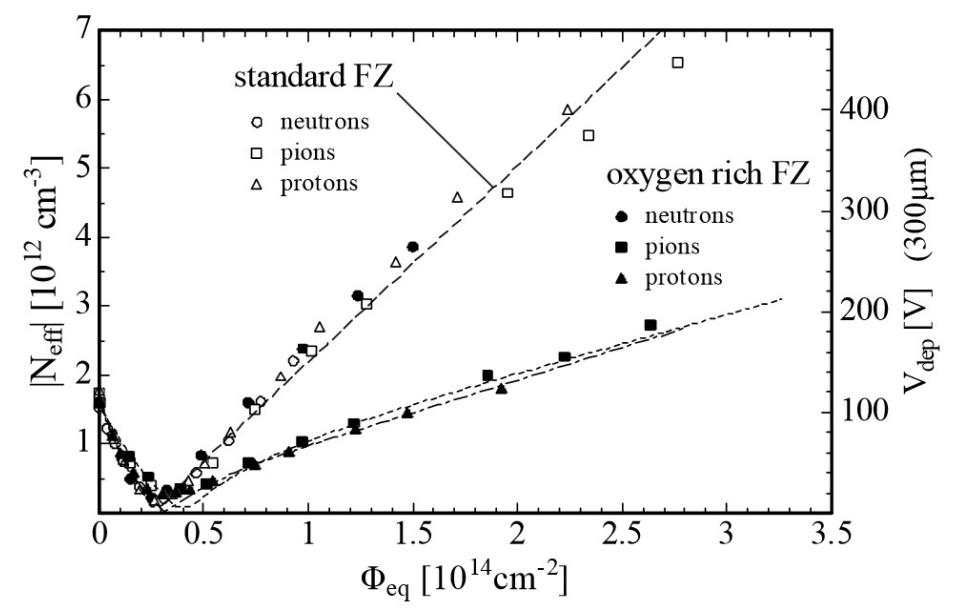

Figure 3.9: Comparison of the effective doping concentration/depletion voltage as a function of $1 \mathrm{MeV}$ neutron equivalent fluence for standard and oxygen enriched float zone silicon. Especially for fluences above the type inversion point, the advantages in terms of depletion voltage for oxygenated silicon are clearly visible [36].

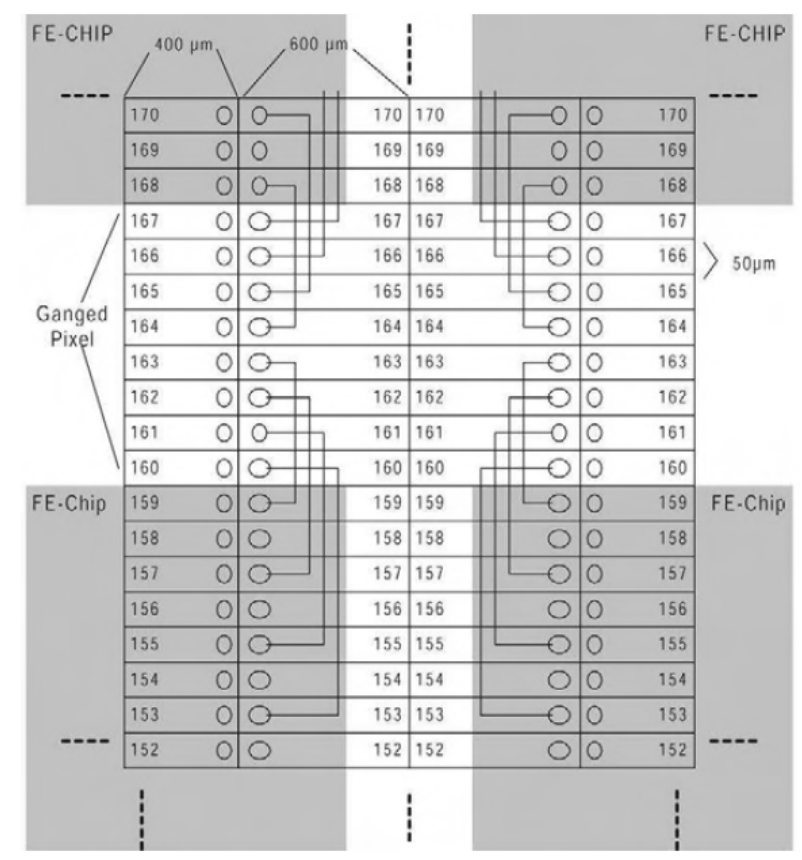

Figure 3.10: Schematic of the sensor layout in the region between four readout chips. Ganged pixels are used to cover the inter-chip region [37].

the whole analogue chain can be tuned. Several DACs ${ }^{1}$ are available for the tuning procedure, where some can be set individually for each pixel, others are set globally for the whole FE chip. Figure 3.13 illustrates the response behaviour for different injected charges. For higher injected charges, the rising edge of the preamplifier output is steeper, than for lower charges. This effect is called timewalk and can explain slightly varying signal detection times in synchronized data taking mode. More details on the FE-I3 chip can be found in [40] and [38].

\footnotetext{
${ }^{1}$ Digital to Analog Converter
} 
n-in-n detectortype
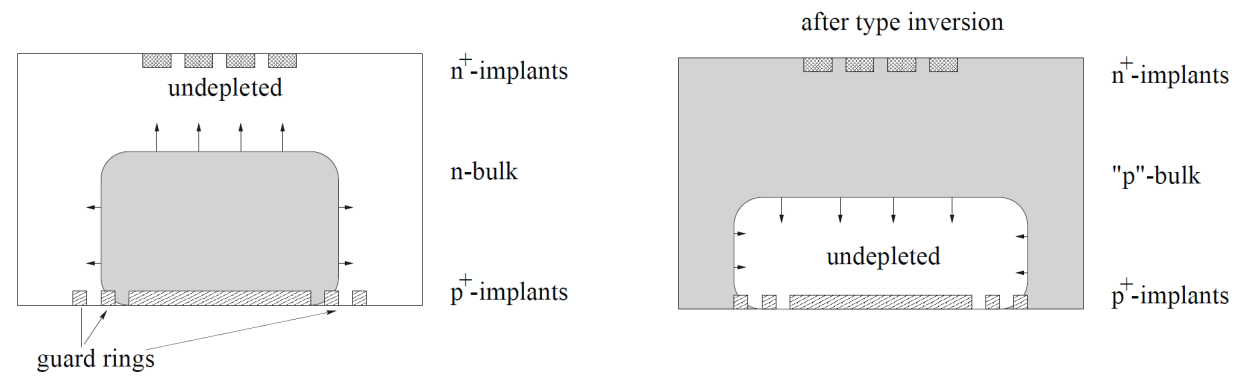

Figure 3.11: Schematic drawing of the depletion region before and after irradiation for $n$-in- $\mathrm{n}$ sensors in underdepleted operation [35].

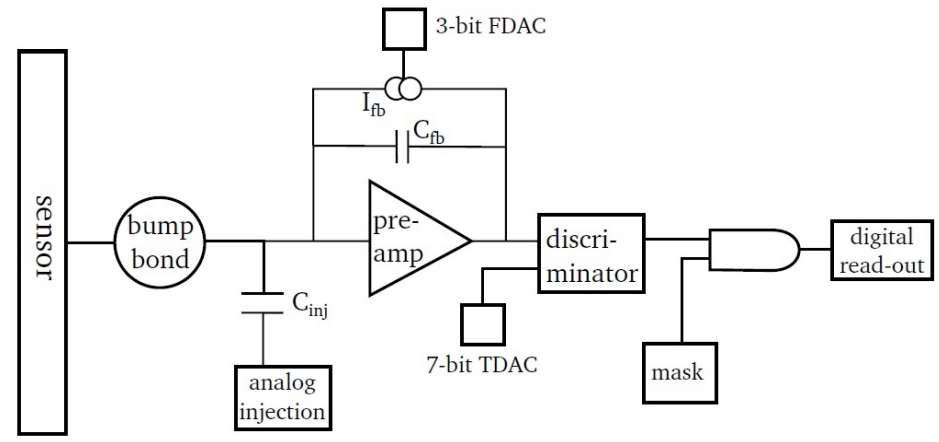

Figure 3.12: Simplified schematic of the analogue part of the FE-I3 chip, including a bump bond, test circuits and the preamplifier and discriminator chain, forming the read out signal [39].

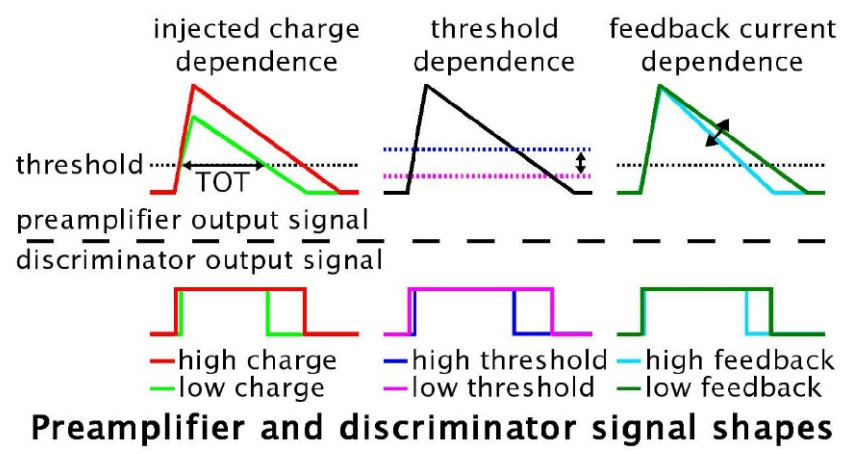

Figure 3.13: Dependencies on the injected charge, the threshold and the feedback current of the time over threshold (ToT) value in the FE chip preamplifier and discriminator [41]. 


\begin{tabular}{|l|c|r|r|r|}
\hline $\begin{array}{l}\text { radiation } \\
\text { type }\end{array}$ & $\begin{array}{c}\text { interaction/ } \\
\text { scattering }\end{array}$ & $R_{\max }[\mathrm{eV}]$ & $R_{a v}[\mathrm{eV}]$ & $\begin{array}{r}E_{\min }[\mathrm{eV}] \\
\text { point defect }\end{array}$ \\
\hline \hline electrons & Coulomb & 155 & 46 & 260000 \\
\hline protons & $\begin{array}{c}\text { Coulomb } \\
\text { and nuclear }\end{array}$ & 133700 & 210 & 190 \\
\hline neutrons & nuclear & 133900 & 50000 & 190 \\
\hline $\mathrm{Si}^{+}$atoms & Coulomb & 1000000 & 265 & 25 \\
\hline
\end{tabular}

Table 3.1: Characteristic values for different incident radiation in silicon. All particles are supposed to have an energy of $1 \mathrm{MeV}$. The values are $R_{\max }$ as the maximum possible recoil energy of the incident particle, $R_{a v}$ giving the average recoil energy and $E_{\min }$ point defect the minimum energy that is required to create a point defect in the lattice [28]. 



\section{Chapter 4}

\section{Upgrades of the ATLAS Experiment - The Insertable $b$-Layer and beyond}

\subsection{Upgrades of the ATLAS Experiment}

Currently, the ATLAS detector and all other LHC experiments undergo an upgrade process, making use of the scheduled "long shutdown 1" (LS1) of the LHC [42]. This section focusses on the upgrade activities of the ATLAS pixel system during long shutdowns 1,2, and 3. The main goals for the ATLAS pixel system during LS1 are the insertion of a new fourth pixel layer, called "Insertable b-Layer" (IBL), into the existing detector and an upgrade of the pixel services in the context of the new service quarter panel (nSQP) project. The LHC will not only provide higher centre of mass energies after LS1, but also a higher luminosity (see chapter 2.1.1). The current pixel layers already had to withstand some years of operation and irradiation and have to be operated several more years. To compensate expected efficiency losses and to cope with the higher expected luminosity, a new additional pixel layer is required. Having a fourth pixel layer will not only add redundancy in case of module failures in the current pixel system, but also boost the tracking performance of ATLAS. Due to the smaller radius and smaller pixel sizes compared to the current b-layer, especially vertexing and b-tagging are expected to improve with IBL, due to better impact parameter determination. Separation of vertices will be a bigger challenge after the luminosity upgrade, due to higher expected pile-up. Again, having an additional high precision detector layer closer to the interaction point is of advantage to keep the fake rate caused by random possible cluster combinations low.

As second step within the planned upgrade schedule for the ATLAS experiment, a full replacement of the Inner Detector is scheduled for the long shutdown 3 (LS3), probably starting in 2023 for around 30 months [43]. The machine upgrade during LS3 will provide a new record instantaneous luminosity of at least $5 \times 10^{34} \mathrm{~cm}^{-2} \mathrm{~s}^{-1}$ (design studies including safety factor at $7 \times 10^{34} \mathrm{~cm}^{-2} \mathrm{~s}^{-1}$ ), which is far beyond the design limits of the current systems. For the Inner Detector, not only the radiation damage limits of the current system (including IBL) will be exceeded, but also pile-up will be a bigger problem. Pile-up estimates reach up to 200 collisions per event, which in summary requires new concepts, new designs and an even bigger focus on cost optimization. More details follow in 4.1.2.

\subsubsection{The Insertable b-Layer}

Due to its position closest to the interaction point, the ATLAS pixel detector receives the highest fluence of all ATLAS sub-detectors [44]. As described before, this does not only cause damages within the sensors, but also readout electronics suffers, which results in a decreasing detector performance. Since after the first years of operation and irradiation the current ATLAS pixel detector performs better than expected, the decision was taken to not replace the current pixel b-layer, but to insert a new b-layer between a new smaller beampipe and the existing detector, to compensate for future damages. This also 
gave the name to the project "Insertable b-Layer" (IBL). The new beampipe has a reduced radius of just $3.0 \mathrm{~cm}$ to provide the extra space for the IBL, as shown in drawing 4.1. To prepare the pixel system for this new setup, it was extracted from the ATLAS detector in 2013 and brought to the surface. There, new services were installed and beginning of 2014 the pixel detector was inserted to ATLAS again, prepared for IBL.

When the IBL project was launched, three sensor technologies were competing: 3D silicon, planar

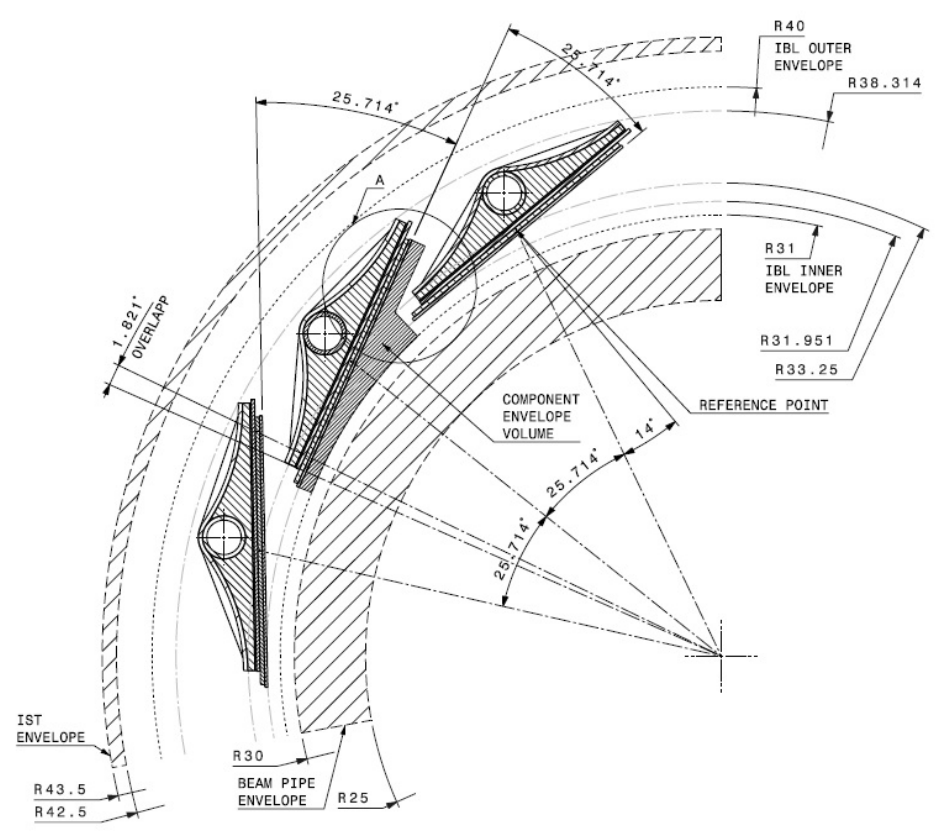

Figure 4.1: Technical drawing of the IBL layout in R $\phi$ view, showing the new beampipe, three staves and the IBL envelopes [42].

silicon and diamond (more details in section 4.2). For all three of them, a set of general requirements was set up, which are:

- geometric efficiency of at least $97.8 \%$, which means an inactive edge width of less than $225 \mu \mathrm{m}$ for single chip assemblies and less than $450 \mu \mathrm{m}$ for double chip assemblies

- sensor thickness between $150 \mu \mathrm{m}$ and $250 \mu \mathrm{m}$, to achieve a low amount of material

- maximum depletion voltage of $1000 \mathrm{~V}$

- maximum power dissipation of $200 \frac{\mathrm{mW}}{\mathrm{cm}^{2}}$ at $1000 \mathrm{~V}$ depletion voltage, where most of the power is caused by the FE chip

- leakage current per pixel of less than $100 \mathrm{nA}$

- operating temperature of the modules $<-15^{\circ} \mathrm{C}$

- after fluence of $5 \times 10^{15} \frac{\mathrm{n}_{\mathrm{eq}}}{\mathrm{cm}^{2}}$ at a depletion voltage of maximum $1000 \mathrm{~V}$ a global in time efficiency of at least $97 \%$ for each module

- pixel size of $250 \mu \mathrm{m}$ x $50 \mu \mathrm{m}$ 
- stable functionality after a fluence of $5 \times 10^{15} \frac{\mathrm{n}_{\mathrm{eq}}}{\mathrm{cm}^{2}}$ non ionizing energy loss (more relevant for sensor) and 250 Mrad total ionizing dose (more relevant for FE chip)

- the whole detector is organized in 14 staves

- for signal processing the FE-I4 chip is used, which is organized in 80 columns and 336 rows

To achieve the required geometric efficiency, the inactive edges of the sensor had to be shrunk, compared to the current ATLAS pixel sensor design. The reason is the limited space between new beam pipe and existing b-layer, which does only allow a slight sensor overlap in $\phi$-direction, but no shingling in z-direction anymore, as figure 4.2 illustrates. As a consequence studies for sensor designs with different edge widths and different numbers of guard rings had to be performed. Another aim was to target for just $0.015 \mathrm{X}_{0}$ at perpendicular incidence for IBL modules, compared to $0.03 \mathrm{X}_{0}$ for current ATLAS pixel modules. So the best compromise between a thin and a mechanical stable sensor had to be found. Together with a new sensor, also a new FE chip had to be developed.

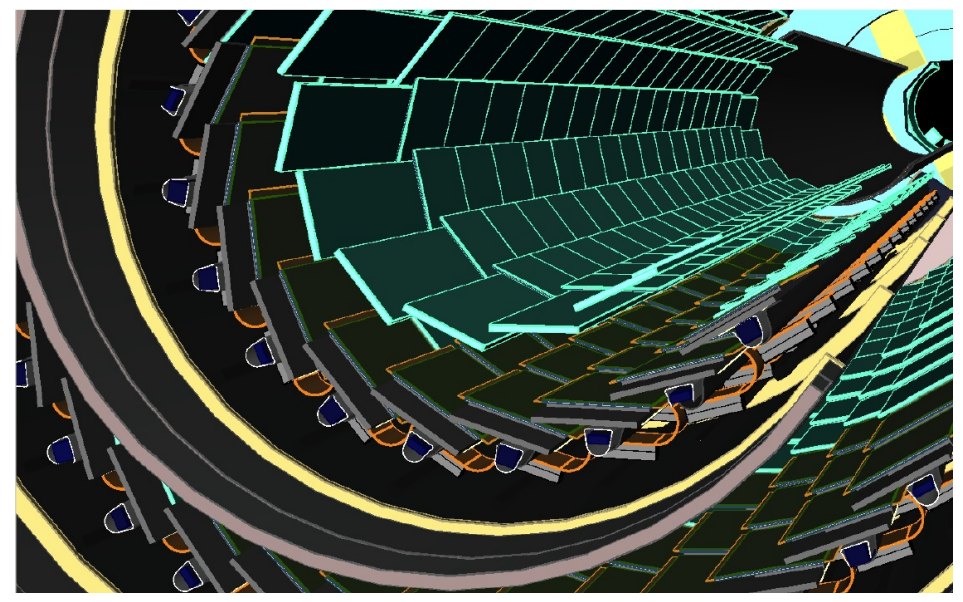

Figure 4.2: Rendered view in z-direction of the ATLAS Insertable b-Layer inside the existing inner pixel system layer, showing the arrangement of support structures and sensors. The picture especially shows that the IBL arrangement allows a small sensor overlap in $\phi$-direction, but not in $\mathrm{z}$-direction due to the limited space [42].

Compared to the current pixel system readout chip FE-I3, produced in a $250 \mathrm{~nm}$ CMOS process and designed for a standard pixel size of $400 \mu \mathrm{m} \times 50 \mu \mathrm{m}$, the IBL readout chip had to be designed in a more radiation tolerant way and with smaller pixel sizes of $250 \mu \mathrm{m} \times 50 \mu \mathrm{m}$. A reduction of the pixel pitch in the shorter direction was not possible, since $50 \mu \mathrm{m}$ is close to the minimal pitch required for bump bonding. The production technology was changed to a $130 \mathrm{~nm}$ CMOS process, which is intrinsically more radiation tolerant, while the total area of the chip grew from $7.6 \times 10.8 \mathrm{~mm}^{2}$ (FE-I3, 2880 cells) to $20.2 \times 18.8 \mathrm{~mm}^{2}$ (FE-I4, 26880 cells). A comparison is shown in figure 4.3. A bigger FE chip not only helps achieving higher geometric efficiency, but also reducing costs, since handling and processing costs scale per device, rather than size.

Going to the smaller feature size of $130 \mathrm{~nm}$ makes the chip more radiation tolerant due to a thinner gate oxide layer, such that it is rated up to the desired value of $250 \mathrm{Mrad}$ [45]. One of the most dramatic changes in the readout architecture of the FE-I4 chip, compared to the I3 generation, is the pixel matrix structure of the FE-I4. While for the I3 generation the architecture was based on a column drain readout, where trigger logic and data storage was placed peripheral, data storage in the I4 generation is realized 


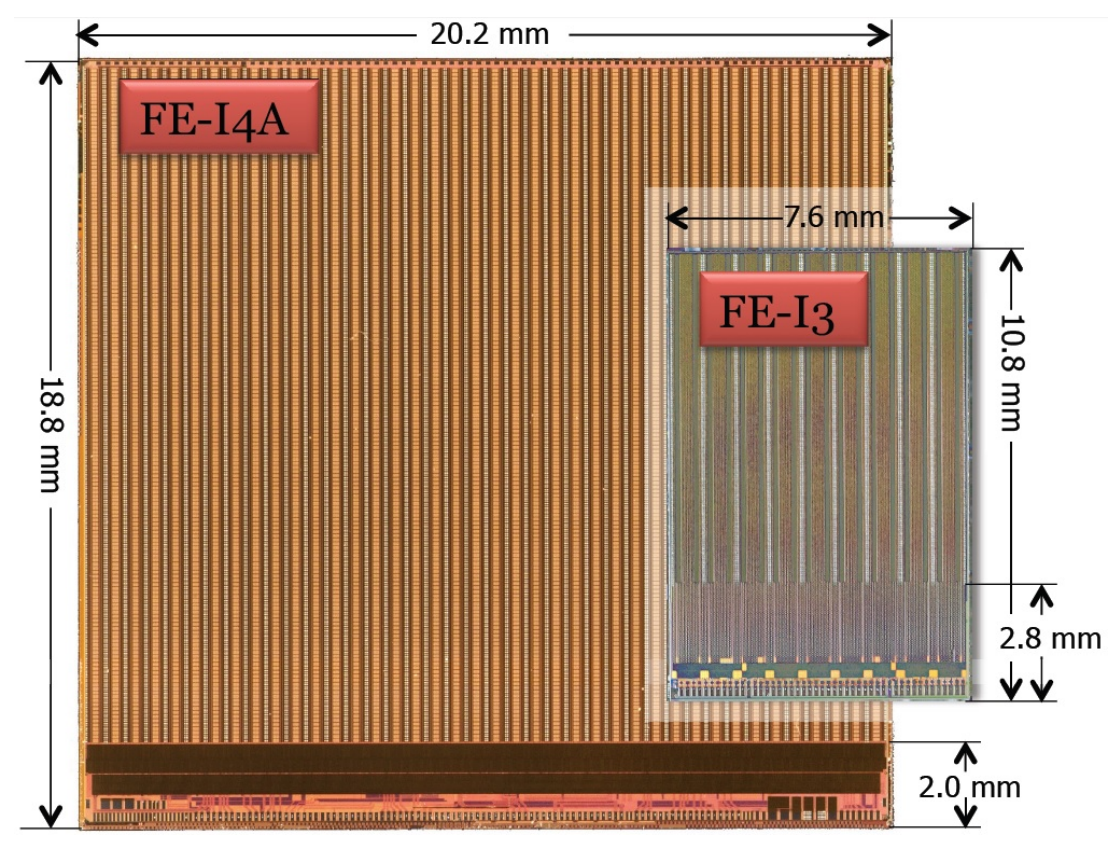

Figure 4.3: FE-I4A chip, as used for IBL module testing and to scale FE-I3 chip, used in the current pixel detector. In comparison, the new chip grew significantly in size and number of pixel cells, while being produced in a more radiation tolerant process [44].

locally in the pixel cell. Data is kept there until a trigger signal arrives and then read out. Figure 4.4 illustrates these features in a schematic sketch.

Simulations have shown an efficient readout using this new FE chip generation at IBL radius up to luminosities of $3 \times 10^{34} \mathrm{~cm}^{-2} \mathrm{~s}^{-1}$. This can be reached not only because of the new readout structure, which removed a bottleneck compared to the FE-I3 structure, but also because the readout speed was upgraded from $40 \mathrm{MHz}$ (FE-I3) to $160 \mathrm{MHz}$. These radical changes of course also require new designs on the whole data acquisition chain. More information can be found in [42] and [46].

\subsubsection{Upgrades for the High-Lumi LHC}

In the long term plans during the years 2023-2025 another long shutdown is scheduled, lasting for 30 months. This time slot will be used by the experiments to perform upgrades. For the ATLAS experiment, the upgrade will include a full replacement of the Inner Detector, to cope with the new machine performance, delivering world record instantaneous luminosities [43], which also gives the name to the project "High Luminosity LHC" (HL-LHC). The letter of intent states a planned integrated luminosity of $2500 \mathrm{fb}^{-1}$ over ten years. This huge additional dataset gives access to extremely rare physics events and provides the possibility to verify or falsify further theories (see chapter 2.2).

On the detector side, a replacement of the ATLAS Inner Detector is required, where up to a radius of $\sim 30 \mathrm{~cm}$ a four layer silicon pixel system is planned, including six end-caps on either side. For radii larger than $\sim 30 \mathrm{~cm}$ a new silicon strip detector is planned. The current Inner Detector is not capable to fulfil the specifications after the LS2 upgrade, neither in terms of radiation, nor in hit occupancies. A draft of the new Inner Detector layout for the time beyond 2025 is shown in figure 4.5.

Summing up the foreseen pixel areas gives new dimensions in area and number of channels for the planned pixel detector, as provided in table 4.1. Combining the foreseen layout with the upgraded performance of the HL-LHC specifications, means also an increase in the expected fluence to values of 


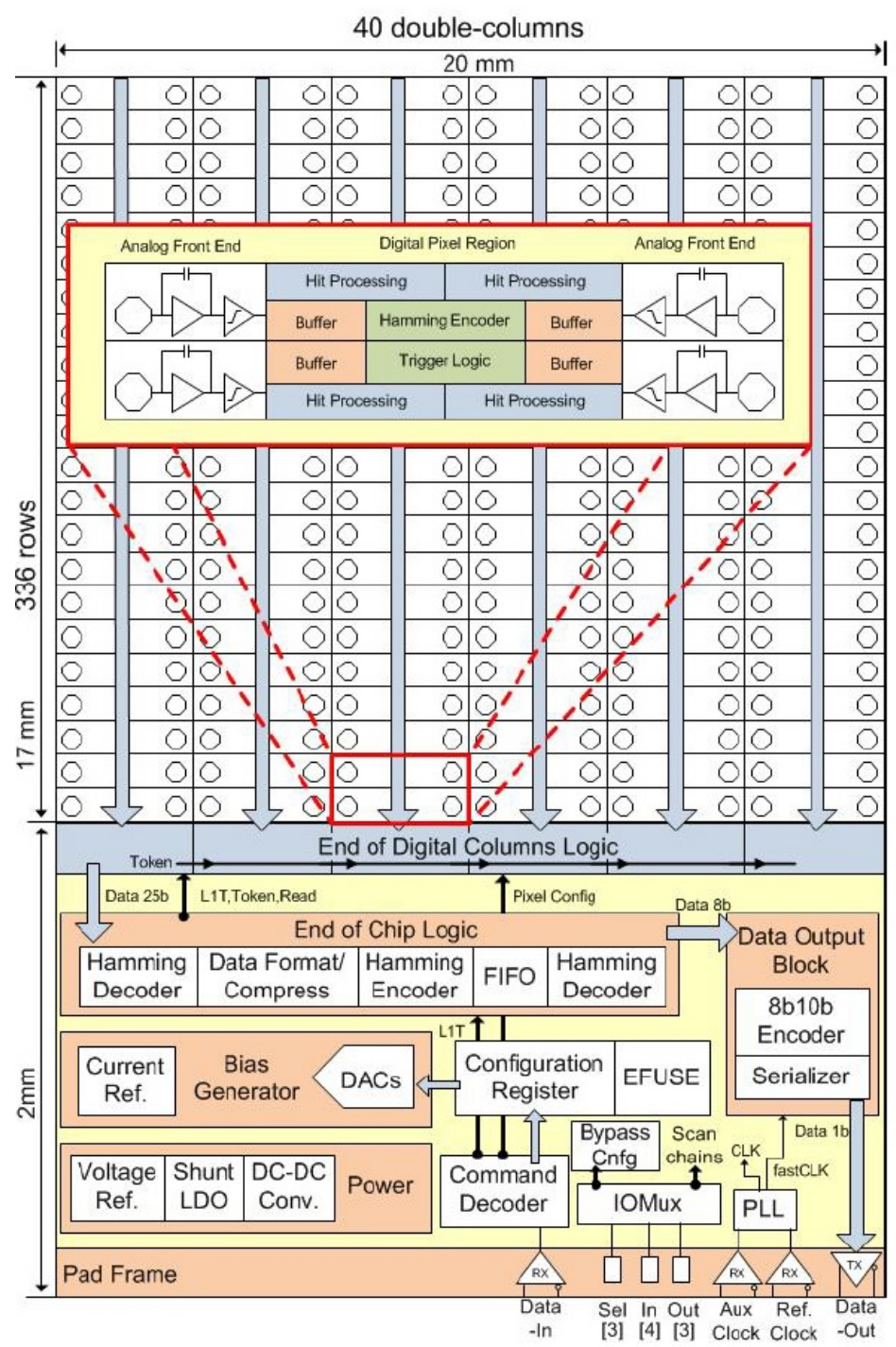

Figure 4.4: Sketch of the pixel logic and peripheral structures in the bottom $2 \mathrm{~mm}$ of a FE-I4A chip [44].

$1.4 \cdot 10^{16} \frac{\mathrm{n}_{\mathrm{eq}}}{\mathrm{cm}^{2}}$ for the innermost layer. Thus, for future developments the focus has to be on even more radiation tolerant pixel modules, but with the same importance on cost optimization due to the sheer dimensions of the future pixel project. 


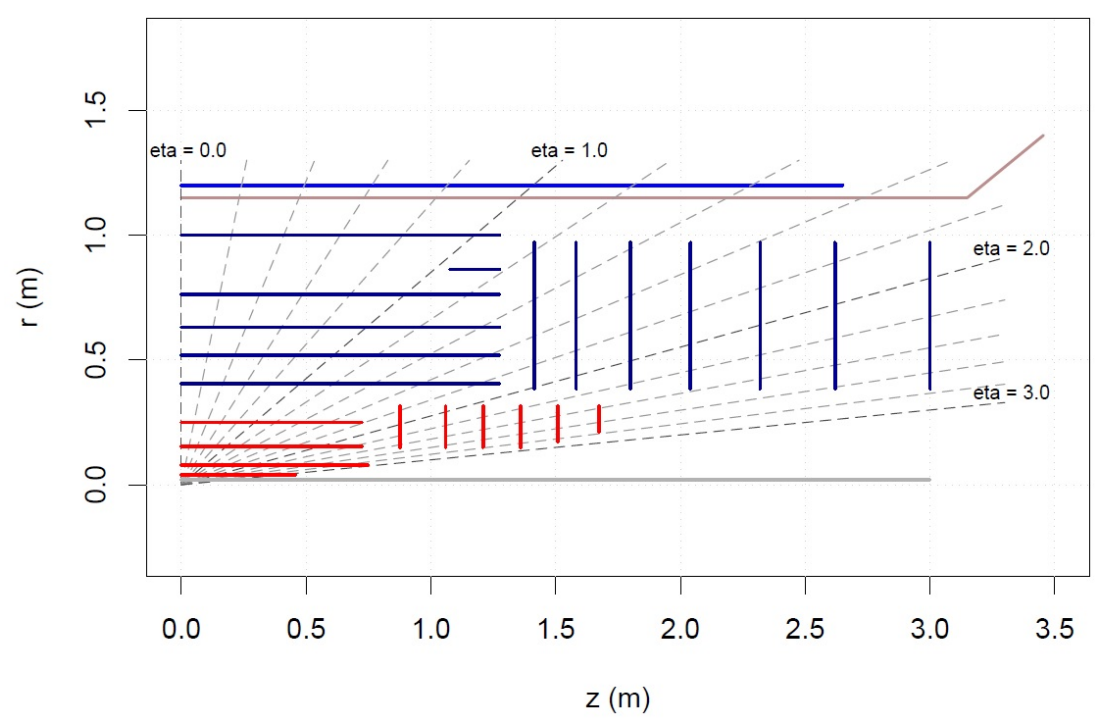

Figure 4.5: Baseline layout for the new all silicon tracker after the phase2 upgrade. In red four barrel layers and six end-caps per side are shown for the pixel system, in blue the new strip detector [43].

\begin{tabular}{|l|c|c|}
\hline detector & area $\left[\mathrm{m}^{2}\right]$ & channels $\left[10^{6}\right]$ \\
\hline \hline pixel barrel & 5.1 & 445 \\
\hline pixel end-cap & 3.1 & 193 \\
\hline sum & 8.2 & 638 \\
\hline
\end{tabular}

Table 4.1: Benchmark data for the planned pixel detector in the context of the upgrade towards the High Luminosity LHC [43]. 


\subsection{Sensor Technologies}

In the planning phase of the IBL project, three sensor technologies were considered [46]: 3D silicon sensors, planar silicon sensors and diamond sensors. Since the original project schedule of IBL was shortened by several years, diamond faded out as potential sensor candidate, due to a lack of available sensors. A significant difference between the handling of planar and 3D silicon sensors is the IBL module format. While planar sensors are mounted as "double chip modules", where each sensor has the size of two FE-I4 chips, 3D sensors are one chip modules combined to a unit of two chips (see figure 4.6). To avoid any damages on neighbouring modules due to high voltage sparks, an air gap between each two modules is included in the stave ${ }^{1}$ design. The final geometrical parameters of the IBL modules are shown in table 4.2.

\begin{tabular}{|l|c|c|}
\hline structural part & planar module & 3D module \\
\hline \hline gap b/w modules & $205 \mu \mathrm{m}$ & $205 \mu \mathrm{m}$ \\
\hline sensor thickness & $200 \mu \mathrm{m}$ & $230 \mu \mathrm{m}$ \\
\hline module width (along $\mathrm{z}$ ) & $41315 \mu \mathrm{m}$ & $20450 \mu \mathrm{m}$ \\
\hline
\end{tabular}

Table 4.2: Summary of the geometrical parameters for pixel modules to be built for the ATLAS Insertable bLayer [46].
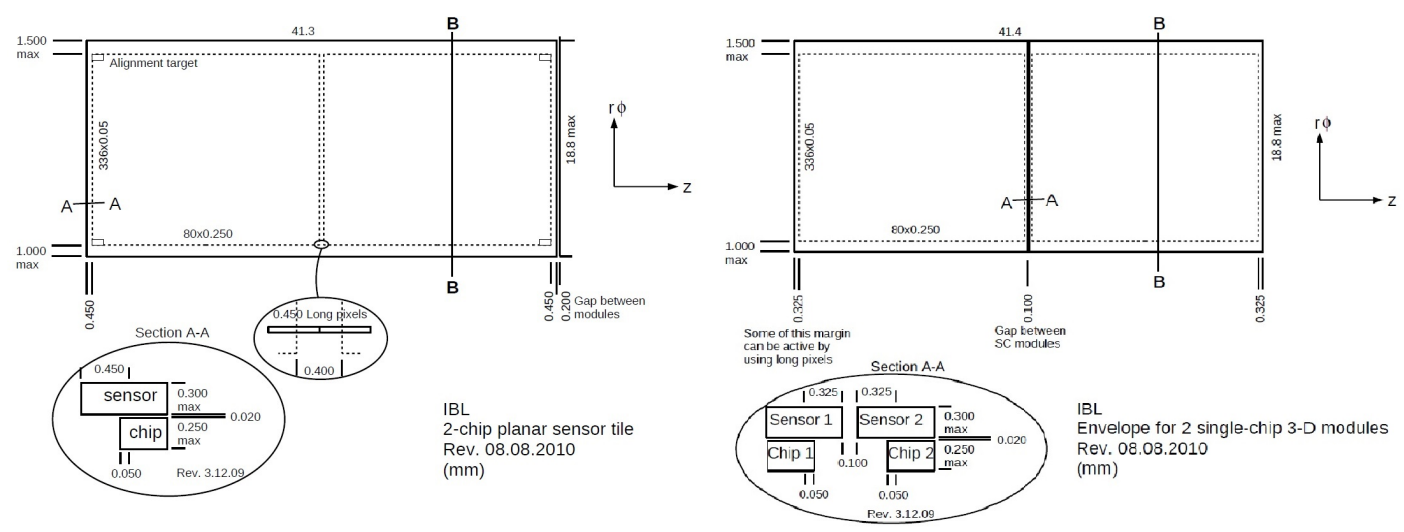

Figure 4.6: Left: IBL double chip module format for planar silicon sensors. Each sensor has the size of two FE-I4 chips and is arranged with extended pixels in the inter-chip region, to have a sensitive area in the central part. Right: IBL single chip module format for 3D silicon sensors. The sensor size corresponds to the size of a FE-I4 chip [42].

For all sensor candidate technologies, the major challenges were the development of radiation tolerant sensors up to a fluence of $5 \times 10^{15} \frac{\mathrm{n}_{\mathrm{eq}}}{\mathrm{cm}^{2}}$ and beyond, the requirement to match the geometrical efficiency without sensor overlaps in z-direction and to have highly efficient sensors after irradiation with a maximum voltage of $1000 \mathrm{~V}$ [42]. For comparison: the current planar silicon ATLAS pixel modules can be operated up to a fluence of $\sim 2 \times 10^{15} \frac{\mathrm{n}_{\mathrm{eq}}}{\mathrm{cm}^{2}}$, have an inactive margin of $1 \mathrm{~mm}$ and can be depleted with a maximal voltage of $700 \mathrm{~V}$.

In the following sections an overview on all three IBL candidate technologies is given.

\footnotetext{
${ }^{1}$ arrangement of modules as staves, where a stave has the length of the IBL barrel cylinder and the width of one module
} 


\subsubsection{D Pixel Sensors}

Compared to planar sensors, the charge collection in 3D sensors happens in the perpendicular direction, namely between doped columns, parallel to the sensor surface [47]. To achieve this, the doped columns are oriented from the front- to the backside of the sensor. This is also the main advantage of the 3D technology, as shown in figure 4.7. The distance between the columns is on the order of $50 \mu \mathrm{m}$, which

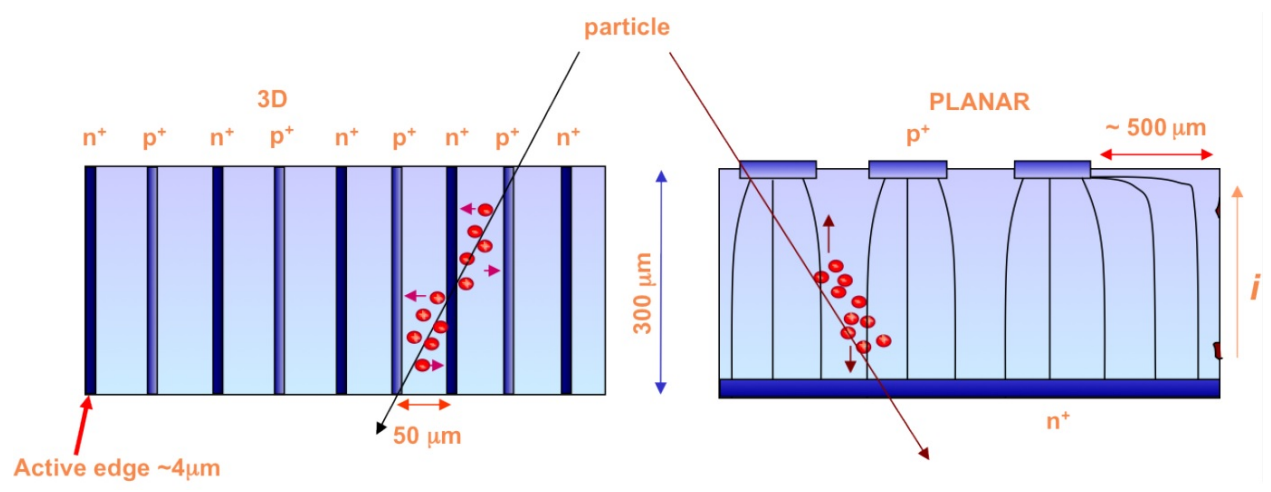

Figure 4.7: Left: schematic of a particle crossing a 3D silicon sensor. The created charge carriers drift in the horizontal direction, with a maximum drift distance of several $10 \mu \mathrm{m}$. Right: the same situation for a planar sensor, where the charge carriers drift a maximum distance of few $100 \mu \mathrm{m}$. In both cases the drift values naturally depend on the exact design [48].

consequently is equal to the maximum drift distance for charges from the point they were created to the readout. As a result, not only the signal readout happens faster compared to planar sensors, but also the sensors need a lower depletion voltage - due to the shorter distance between the electrodes - and have an intrinsic radiation tolerance, because the probability for charge trapping is lower on the shorter distance. Due to the sensor design, especially the depletion voltage after irradiation is just on the order of few $100 \mathrm{~V}$, compared to around $1000 \mathrm{~V}$ for planar sensors. This also means a low leakage current, which is advantageous in the sense of power dissipation during detector operation. All of the previous features promised a good performance, especially after irradiation. Thus, the expected most probable collected charge values were simulated for the candidate design specifications, as shown in figure 4.8. Even with high irradiation doses, the simulated collected charge did not decrease dramatically, which was another strong argument for investigating 3D devices in more detail. The number of columns per pixel is a compromise between an increased level of capacitive noise in the sensors with increasing number of columns and the charge collection efficiency. Beside the noise argument, also the columns themselves are not fully active, especially under perpendicular incidence. In case of IBL, all modules are mounted with a slight tilt of $14^{\circ}$, which settles the problem as long as the projected area of the columns just covers a small fraction of the sensor surface area. Measurements with the previous FE-I3 chip and 3D sensors showed that this compromise should be matched best in the down-scaled so called "2E-250" [42] configuration, equipped with the FE-I4 chip [49]. This means that in the sensor volume of one pixel cell two n-type electrodes are placed, while the pixel pitch in the long direction is $250 \mu \mathrm{m}$, which matches the IBL specification. The name "3D sensor" originates from the sensor design, which is shown for the IBL candidates in figure 4.9. The best suiting design thickness for 3D sensors was found to be $230 \mu \mathrm{m}$. As figure 4.9 already indicated, two different 3D sensor designs were investigated as candidates for the IBL project:

- "double sided 3D":

One of the major differences of the double sided 3D design, compared to full 3D, beside the 


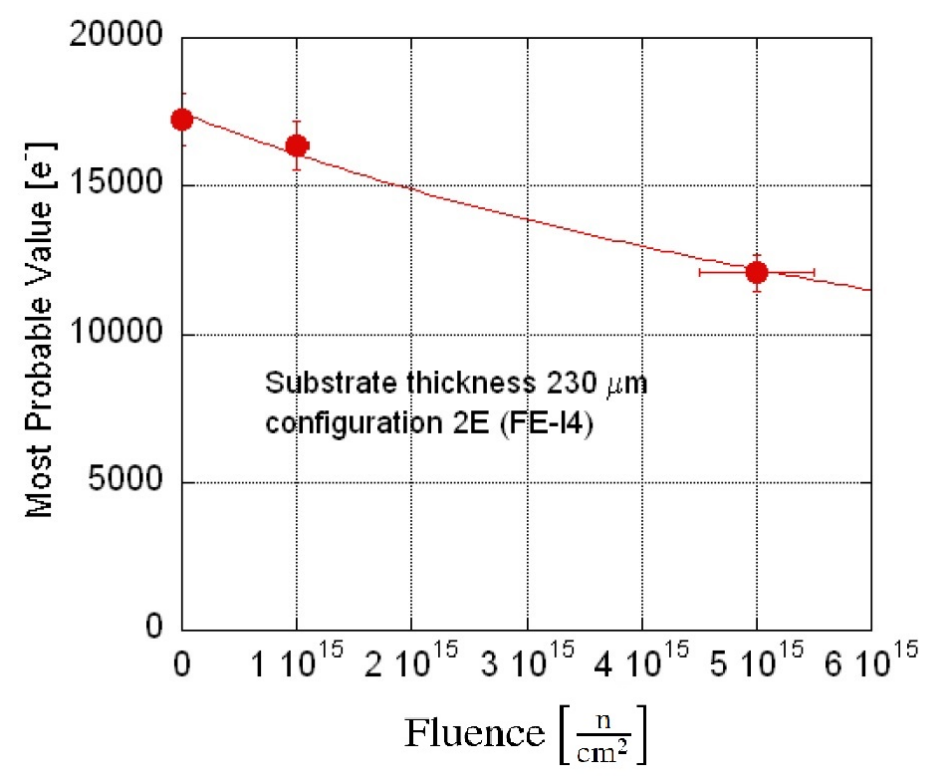

Figure 4.8: Simulated most probable value for the collected charge, using a 3D sensor with the IBL candidate specifications, which are a thickness of $230 \mu \mathrm{m}$ and a 2E-250 electrode configuration [42].

$\mathrm{n}^{+}$etched and filled from top

(a)

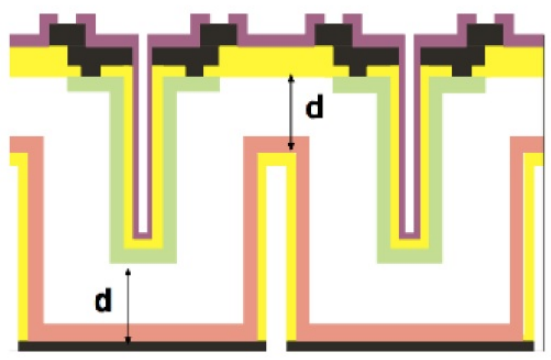

$\mathrm{p}^{+}$etched and filled from bottom
All holes etched and filled from top side

(b)

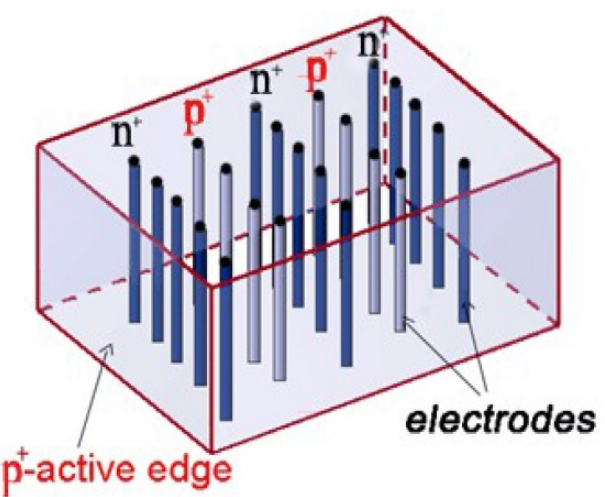

Figure 4.9: Left: 3D sensor design, based on a double sided production, where the columns do not reach through the whole sensor thickness. A fraction $d$ (around $20 \mu \mathrm{m}$ ) of the sensor thickness is not equipped with columns. Right: full 3D sensors. The columns of both doping types reach through the full sensor thickness [42].

production process itself, is the absence of active edges. The production of active edges requires the usage of a support wafer, which is done for the full 3D design, but not suitable for the double sided production process. To match the IBL requirements in terms of geometric efficiency, the double sided 3D sensors were produced with slim edges. The edges in the doubled sided design are equipped with additional columns ("guard fence columns") outside the edge pixel to have a $3 \mathrm{D}$ counterpart to the guard rings that are used in planar designs [50].

- "full 3D":

As mentioned above, compared to the double sided 3D sensors, the full 3D devices have an active 
edge. To realize this feature, a trench is etched into the sensor wafer, using the process that is also used for the sensor production itself. This trench is doped afterwards, such that it finalizes the electric field arrangement of the sensors. This allows to realize inactive regions of only few $\mu \mathrm{m}$ width, which is very advantageous, especially for the IBL arrangement. For the full 3D with active edge design, the high voltage is applied on the FE chip side. The voltage supply is realized through a supply pad, which is placed on the opposite edge of the FE chip readout pads. To avoid these extra bias pads, research has been started in through silicon vias, which can be used to route the voltage through the sensor.

\subsubsection{Planar Pixel Sensors}

In case of planar silicon sensors, the development towards IBL design had several approaches. The baseline design was again the n-in-n design, as for the present pixel detector, but also n-in-p sensors were investigated. To reduce the inactive area, the number of guard rings had to be reduced, while the sensors had to hold higher voltages. In general, the inactive edge width had to be reduced, since the design requirements allowed at most an edge width of $450 \mu \mathrm{m}$. Another general task was to prove the performance with the new FE-I4 chip.

The RD50 collaboration studied intensely the charge collection of silicon microstrip detectors at high fluences [51, 52]. As important result it was found that current design n-in-p microstrip devices showed sufficient charge even after fluences of $2 \times 10^{16} \frac{\mathrm{n}_{\mathrm{eq}}}{\mathrm{cm}^{2}}$ with a high enough electric field. Since the voltage limit for IBL was set to $1000 \mathrm{~V}$, it was investigated how thin sensors can be produced and operated, because at a fixed voltage the electric field strength increases, if the devices are thinner.

During the planning phase, it was decided to investigate for the planar scenario diffusion oxygenated float-zone (DOFZ) silicon sensors with both, n-type (n-in-n) and p-type (n-in-p) bulks. The advantage of using a p-bulk sensor is, that during the processing step, all structures which require a mask (e.g. pixel implants, guard rings) are on the FE chip side. The n-in-n sensors require a double-sided processing with masks, since the guard rings are located on the back of the sensor. On the other hand, a drawback of $n$-in-p sensors is the presence of a significant fraction of the applied high voltage on the FE chip side. Thus, it has to be ensured that a reliable passivation is applied, to protect the FE chip from any high voltage sparks, which would damage the chip.

Finally, for the IBL qualification three different approaches for planar silicon sensors were followed [42]:

- "conservative n-in-n design":

As the name suggests, the conservative design stays as close as possible to the previous ATLAS pixel design. It is based on $n$-in-n sensors with a reduced number of guard rings (reduced from 16 in the ATLAS pixel design to 13 for the IBL candidate, figure 4.10 a)). This was necessary to match the $450 \mu \mathrm{m}$ limit for the maximum edge width. Measurements showed that this design can be operated at full depletion voltage and at least $150 \mathrm{~V}$ beyond that, due to the high conductivity of silicon. Compared to the sensors in the current pixel detector, the pixels were shortened to $250 \mu \mathrm{m}$ length in z-direction, to suit the FE-I4 chip measures. As main advantage of the conservative design, the well known properties of the approach can be stated, since it is very close to the design that was used for the previous pixel detector construction.

- "slim edge n-in-n design":

For n-in-n designs the guard rings are located on the backside of the sensor, which is the p-type region. That allows to shift the guard rings partially into the edge pixels, as shown in figure 4.10 b). Having the guard rings within the pixel area naturally changes the shape of the electric field, 


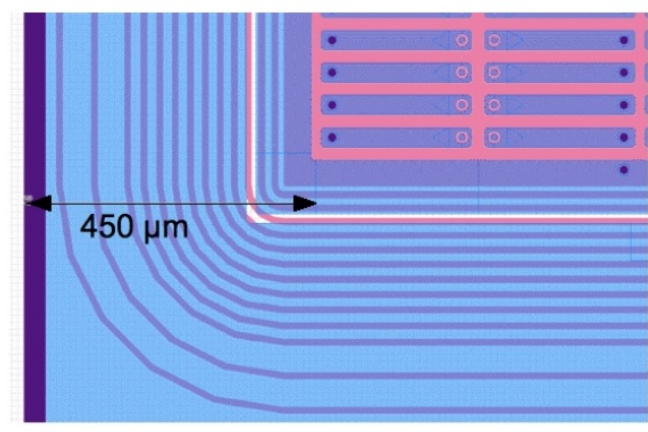

(a)

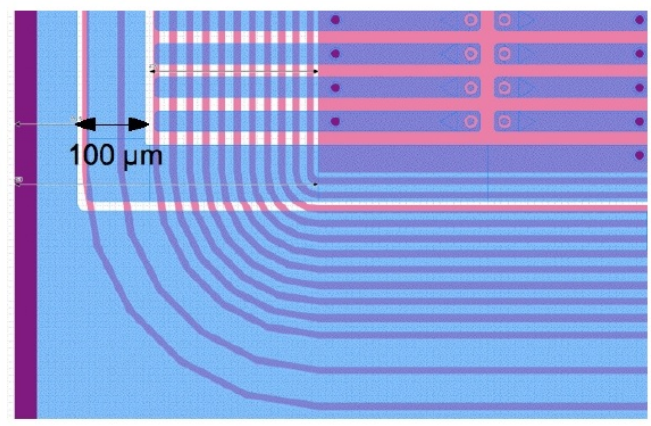

(b)

Figure 4.10: Two different planar n-in-n silicon design candidates, as they were investigated for IBL. Figure a) is the edge region schematic of a "conservative" design, where the number of guard rings is reduced from 16 (current ATLAS pixel sensor) to 13 and the edge width reduced to $450 \mu \mathrm{m}$. Part b) shows the edge region of a "slim edge" design. Most of the guard rings are overlapping with the outermost pixels, which allows to further reduce the inactive part. Since the total dimensions of a sensor are fixed, the outermost pixels are extended to a length of $500 \mu \mathrm{m}$. The extra length covers the region which is inactive in the conservative design [42].

especially close to the edge. But since the guard rings are located on the backside of the sensor, the initial charge collection losses were simulated to be small enough, compared to the expected signal. Taking into account the expected charge collection efficiency losses close to the edge, the signal is still sufficiently high, compared to the threshold. After irradiation the depletion zone anyway does not extend through the whole sensor anymore, but grows from the implant side only to a certain depth. Thus, the effect of a changed field geometry gets even smaller with irradiation. All in all, the slim edge design has an inactive edge width of $100 \mu \mathrm{m}$, which increases the geometric efficiency by $0.5 \%$, compared to the conservative design.

- "thin n-in-p design":

The third design alternative for IBL were n-in-p sensors. As already mentioned, this technology has the advantage that the sensors can be produced in a single sided process, which is cheaper, compared to the double sided process for n-in-n sensors. For future upgrades of the silicon strip system, n-in-p sensors are more or less set as the technology of choice. Since nowadays a large number of vendors for $n$-in-p sensors is available, a big research effort in this technology was not only performed for the IBL upgrade, but is ongoing for future upgrades. To avoid high voltage sparks towards the FE chip, a layer of benzocyclobutene (BCB) is deposited on the sensor surface, on top of the standard oxide and nitride passivation layers. A further advantage of $n$-in-p sensors is the possibility to produce them very thin, using a handle wafer during production. Sensors down to a thickness of $75 \mu \mathrm{m}$ were produced, which is very advantageous in terms of material budget. The candidate n-in-p design for the IBL project is shown in figure 4.11.

For both cases, the 3D silicon sensor designs and the planar silicon designs, all of the above mentioned device types were investigated. The focus in all cases was on the questions whether all vendors are able to produce devices in the required quantity and quality, how the devices perform with increasing irradiation levels and which are the perfect operating conditions. Especially the required high voltages, the operability in the foreseen temperature range and the performance with the FE-I4 chip were of in the focus of the development. In this context, the hit efficiency and the recorded charge signals, as a function of the fluence were of big interest for the final decision for the IBL sensor types. A summary 


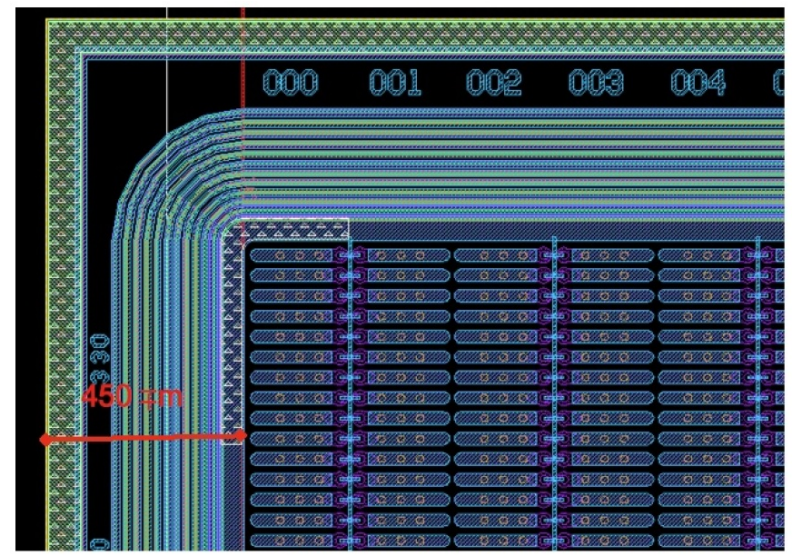

Figure 4.11: IBL candidate planar n-in-p silicon design with an inactive edge width of $450 \mu \mathrm{m}$ [42].

of these analyses from the above mentioned development approaches to the final IBL sensors is given in chapter 7.

\subsubsection{Diamond Beam Monitor}

During the planning phase of the IBL upgrade, also diamond sensors were taken into account [42]. Diamond as sensor material has some advantages, compared to silicon. The most interesting ones are a very low leakage current and the possibility to operate diamond sensors at room temperature, thus no cooling is needed. Due to the large displacement energy per atom of $\sim 42 \mathrm{eV}$, diamond is intrinsically very radiation tolerant. On the other hand, the required energy to create an electron-hole pair in diamond is a lot higher $(13 \mathrm{eV})$, compared to silicon $(3.6 \mathrm{eV})$. This leads to a lower signal than achieved in a silicon sensor of the same thickness, resulting in an even higher importance of a stable FE chip operation at low signal thresholds. In the planning phase of the IBL upgrade, the diamond candidate design was a poly-crystalline sensor with a thickness of $300 \mu \mathrm{m}$.

Due to the fact that the IBL installation date was pulled forward to the current first long shutdown, which shortened the development time for IBL devices by several years, the supply situation was not sufficient to guarantee sensor availability. Thus, the approach of using diamond sensors for the IBL detector was withdrawn.

Within the ATLAS experiment, as for the other LHC experiments, there is the need to have a reliable beam monitoring system [46]. Currently the beam conditions are measured in ATLAS by the Beam Conditions Monitor (BCM), which is also based on diamond sensors [53]. Due to the necessity for higher granularity because of the increasing luminosity, the new Diamond Beam Monitor (DBM) project was established, based on the research that has been performed for diamond IBL sensors before. The DBM project exploits the extreme radiation tolerance of diamond, which suits the future operating conditions of the DBM, being exposed to fluences of $\sim 2 \times 10^{15} \frac{\mathrm{n}_{\mathrm{eq}}}{\mathrm{cm}^{2}}$ [54]. Due to the upgrade of services for the IBL within the new Service Quarter Panel (nSQP), the DBM detector is already installed in the pit. The installation point of the DBM modules is approximately $1 \mathrm{~m}$ away from the interaction point (in $\mathrm{z}$-direction) and at a radius of $65 \mathrm{~mm}$, as indicated in figure 4.12. On either side of the interaction point, a set of four telescopes was installed, each carrying three sensors, which were mounted on the $10 \mathrm{~cm}$ lever arms. Figure 4.13 shows drawings of the DBM setup. In its foreseen constellation, the Diamond Beam Monitor is expected to have four incident tracks per telescope and bunch crossing [46].

Since the sensors are equipped with the FE-I4 chip, and therefore have a pixel size of $250 \mu \mathrm{m}$ x $50 \mu \mathrm{m}$, 


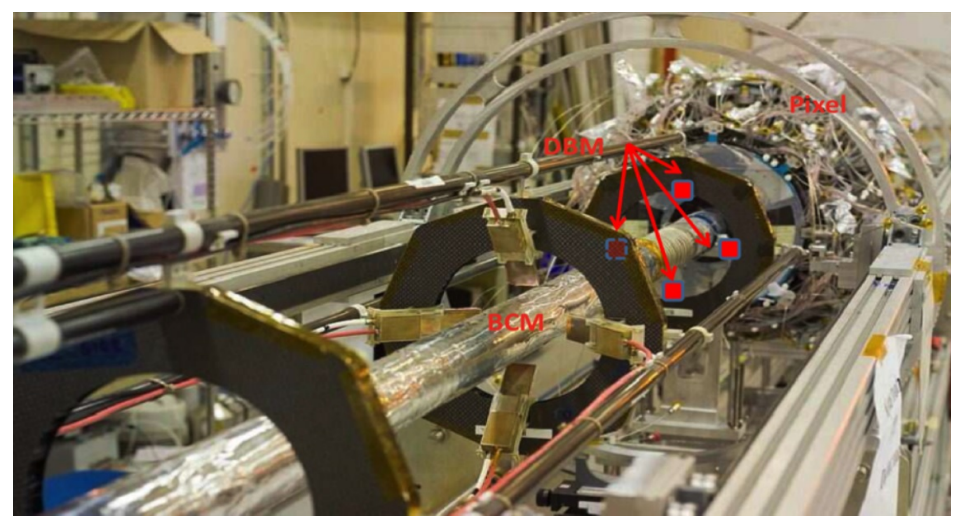

Figure 4.12: Picture of the extracted pixel detector including support structure and services, indicating the positions of the DBM telescopes [54].

combined with a high speed readout, the telescopes are expected to resolve up to ten particle tracks per bunch crossing without ambiguities. This makes the DBM a radiation tolerant beam monitor, which is able to withstand the expected doses and fluences of future upgrades.
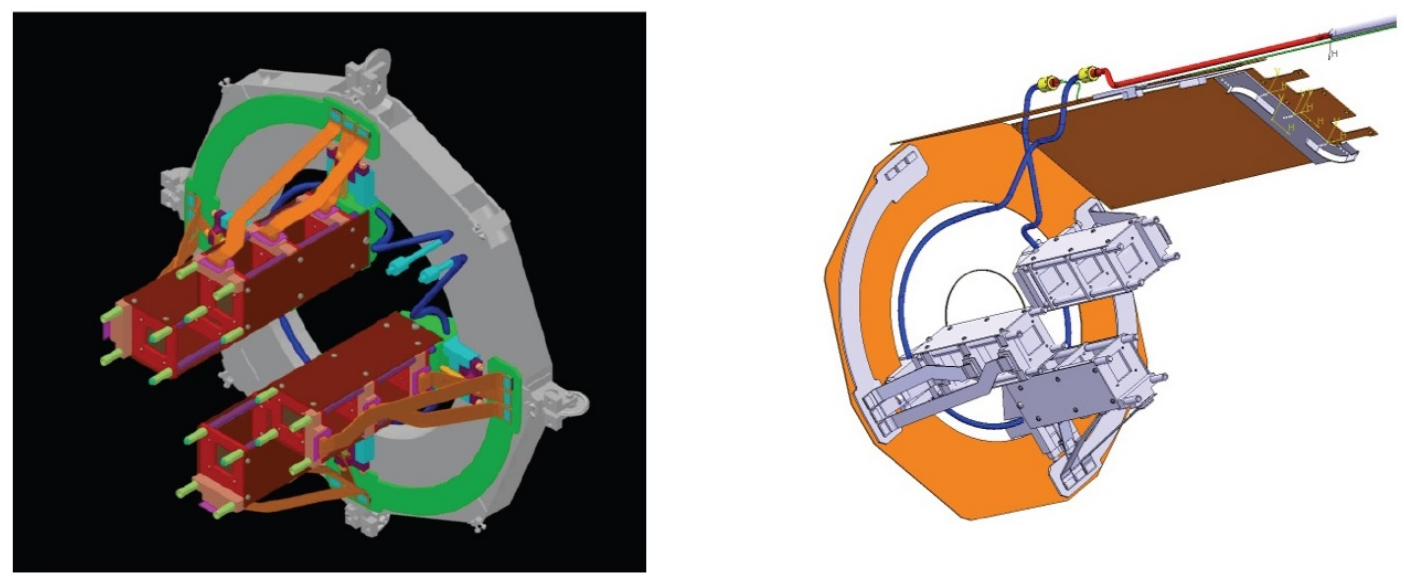

Figure 4.13: Left: CAD drawing of the DBM setup on one side of the interaction point, showing the four telescope arms and the supply cables. Right: DBM cooling structure (in blue) and the connection to the nSQP, on which the Diamond Beam Monitor is mounted [46].

\subsubsection{Technology Choice}

The sensor qualification process for all of the sensor candidates mentioned above happened in several stages. Beside device simulations and lab measurements, an important tool to investigate the device behaviour under realistic conditions are testbeam measurements. Since the geometric layout and especially also the FE chip of all silicon devices and diamond devices is the same, the testbeam measurements were all performed using the same setup, described in section 5. This also allowed to use the package of track reconstruction software and device analysis tool, that was developed for IBL analysis (see section 6) for all IBL testbeam campaigns and for the testbeam measurements for the DBM.

Evidence that the search for the final IBL sensor candidates was successful, is given in figure 4.14, 
which shows two prototype devices. Currently, the final IBL staves are being produced, using both kinds of sensors, the planar double chip devices and the 3D single chips. The reason for using both sensor types is the comparable performance in lab tests and testbeam measurements together with the high production yield and thus good availability of planar and 3D sensors.

\section{Dressed modules}

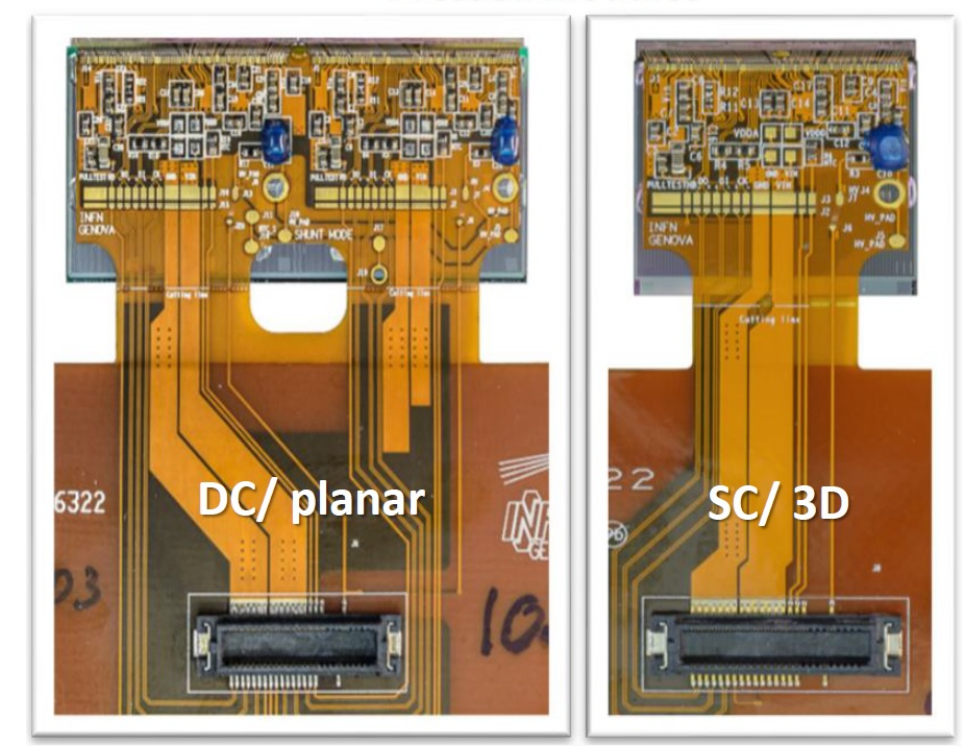

Figure 4.14: Dressed IBL modules [55]. 


\section{Chapter 5}

\section{Testbeam Setup}

\subsection{The ATLAS Pixel Testbeam setup}

For sensor prototype characterization, testbeam measurements are one of the most important techniques to study the sensor performance under realistic conditions. Amongst others, testbeam telescopes provide a sub-pixel resolution for sensor studies and in general a test environment, which allows an easy change of tested devices and measurement parameters. Typically such a telescope is made up out of well known high resolution sensors that are used to reconstruct particle tracks and estimate the particles' incident point on the studied devices with precision of better than one $\mu \mathrm{m}$. The ATLAS pixel community decided to use the EUDET ${ }^{1}$ telescope for IBL testbeam measurement campaigns and sensor developments beyond the IBL project. One of the crucial advantages of the EUDET telescope system was the availability of copies at all testbeam facilities used by the IBL community.

The EUDET telescope [56] mechanically consists of a support structure, that carries the two telescope arms and can be rotated by a few degrees to allow an alignment parallel to the beam axis, see figure 5.1. Each arm is equipped with three Mimosa26 telescope sensor planes (see 5.1.1), which can easily be moved within a range of $64 \mathrm{~cm}$ along the beam axis in total. Here, half of the moveable range is reachable by sliding the whole telescope arm on its base structure rails, which has the advantage that the distance between the planes does not change. The other $32 \mathrm{~cm}$ can be achieved by moving the telescope planes themselves on the arm, which naturally changes the distance between the planes. Auxiliary boards are mounted on top of the sensor planes and act as connection between the telescope sensors and the readout chain. The sensors themselves are located within a protective aluminium support frame, which has openings on the front- and backside in the region where the beam passes through, to avoid putting additional material in the beam. These regions are covered with $25 \mu \mathrm{m}$ thin Kapton foil. In the central position between the telescope arms, the devices under test (DUTs) can be mounted on a X/Y table, which can move the DUTs in $\mu \mathrm{m}$ steps.

\subsubsection{Mimosa Sensors}

The EUDET telescope (and its copies) is equipped with Mimosa26 $6^{2}$ sensors [57-59], which are Monolithic Active Pixel Sensors (MAPS). These sensors fulfil the requirements of a testbeam telescope to provide a single-point spatial resolution in the order of $2 \mu \mathrm{m}$, while the amount of material has to be as low as possible. This especially becomes important for measurements at few $\mathrm{GeV}$ beam energy, like at DESY testbeams. In this beam energy regime, multiple scattering plays an important role, thus, less material means a better telescope performance. The MAPS technology is well established and already during the development time of the EUDET telescope it was possible to produce MAPS with a reasonable area of $1 \times 2 \mathrm{~cm}^{2}$. Following this technology decision, the Mimosa26 sensors were developed and

\footnotetext{
${ }^{1}$ European Union funded DETector R\&D towards the International Linear Collider

${ }^{2}$ Minimum Ionizing MOS Active pixel sensor
} 


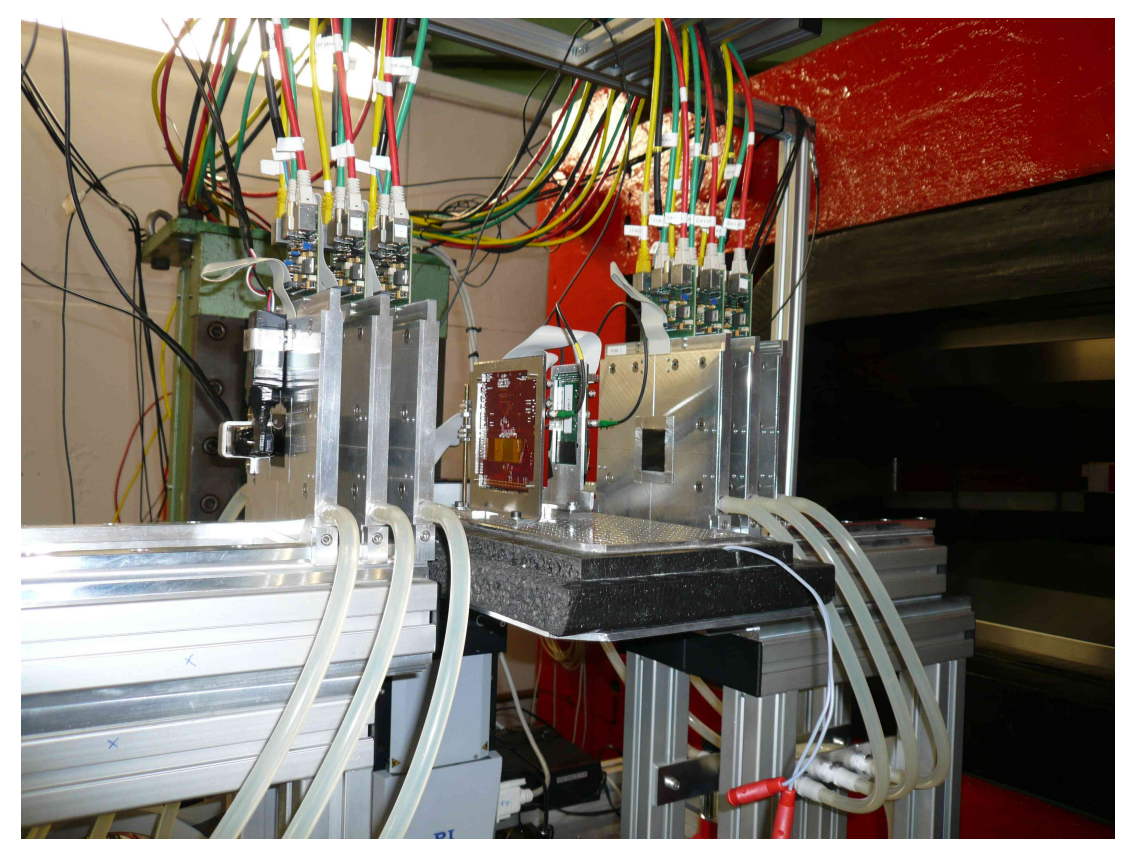

Figure 5.1: The EUDET telescope, set up at DESY for measurements of the ATLAS pixel group. On the left, as well as on the other side of the telescope, two crossed scintillators are mounted for triggering. The two telescope arms, each equipped with three telescope planes, can be seen, as well as the telescope cooling. In the centre the baseplate of the ATLAS pixel cooling box is mounted, with two devices under test on it. For further details, see 5.1 and 5.1.1.

produced at CNRS-IPHC in Strasbourg.

A schematic of a MAPS can be seen in figure 5.2. In contrast to the pixel sensors that are used for the ATLAS experiment, the readout circuits are integrated with the sensors. The sensor itself is a p-doped epitaxial layer with $n+$ implants, which is grown on a $p++$ substrate (higher doping concentration than the bulk region). As can be seen in the schematic, the pixel readout is located on top of the sensor, within the p-well region. In the junction region between the $n+$ implant and the $\mathrm{p}$-epitaxial layer, the charge collection diode is formed, which is also the only depleted region of the sensor. The large differences in the doping concentration of the p-epitaxial layer, compared to the p-well and the $\mathrm{p}++$ substrate, creates potential barriers. This causes the charge carriers produced by an incident particle, to diffuse in the direction of the n-well, which is the charge collection region. Having this layout, the MAPS technology reaches a sensitive pixel area of $100 \%$, since the induced signal can be detected for every incidence point.

This makes MAPS a perfect technology for testbeam telescopes, since the sensors are relatively cheap, due to the widely spread CMOS production, and can be produced with a very thin epitaxial layer, which allows total thicknesses down to few $10 \mu \mathrm{m}$. The biggest disadvantage of this sensor technology is the long readout time, as well as the slow charge collection due to the low fields. A specific feature of the Mimosa sensors are the "self-biased on-pixel" amplifiers, which compensate the leakage current of the pixels and clear the signals after they are read out. Compared to one readout cycle, the latter process is relatively slow, so that signals can remain over several cycles. To avoid a multi-counting of signals, the difference of subsequent signals is calculated, since charge of a previous hit can still be present in a pixel, when the next hit is detected. This method makes a real reset signal unnecessary, since the pixels are ready for a new hit in each state. In total, the Mimosa26 sensors have 1152 columns and 576 rows of 


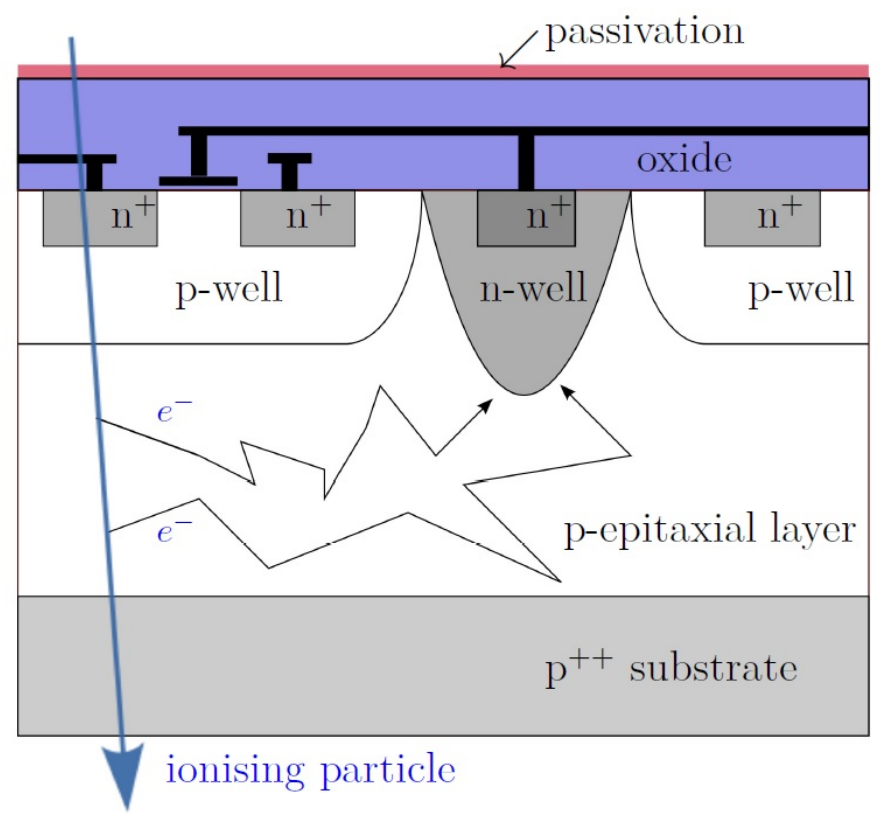

Figure 5.2: Schematic of a Monolithic Active Pixel Sensor (MAPS) in cross section view. The induced charge carriers produced by the incident particle diffuse towards the n-well, where the charge is collected [57].

pixels, where the pixel pitch is $18.4 \mu \mathrm{m}$ by $18.4 \mu \mathrm{m}$. This means an active area of $21.2 \mathrm{~mm} \mathrm{x} 10.6 \mathrm{~mm}$. The readout is zero suppressed and just binary hit information is sampled, so that the amount of data that has to be read out is reduced by a factor of 10 to 1000 . This analogue-to-digital conversion is realized column-wise at the bottom of the pixel array. A full readout cycle of the sensor takes about $112 \mu \mathrm{s}$.

\subsubsection{Devices Under Test}

While in a typical testbeam setup the telescope characteristics are well known and understood, the devices under test (DUTs) are new sensor concepts that are under investigation. These prototype sensors are typically operated in states from perfect conditions to parameters that are far beyond the design values, to predict the device behaviour throughout a whole experiments' lifetime.

In the EUDET setup the (usually up to four) DUTs are mounted between the two telescope arms. In IBL testbeam scenarios some of the first prototypes were equipped with readout chips of the FE-I3 generation, while most data has been collected with FE-I4 devices. The latter design is the one, which the IBL experiment is made out of. Since most of the performed measurements are time sensitive and the integration time of the telescope planes is quite long, compared to the $400 \mathrm{~ns}$ buffer of the ATLAS pixel DUTs, one of the DUTs is used as a reference plane in typical IBL testbeam setups. Here, the reference device had to be an unirradiated, well understood and perfectly operating device. More details about the tested devices can be found in sections 3.2 and 4.2. Since the DUTs are sensitive to light and heat, they were mounted in a custom made polystyrene DUT box (see figure 5.3).

\subsubsection{Data Acquisition Hardware}

While the telescope planes have integrated readout cards, the DUTs can be connected to different readout systems. These systems have to be integrated into the data acquisition chain. For the ATLAS pixel devices, this has been realized for the USBpix system and the RCE system. Both are explained in the 


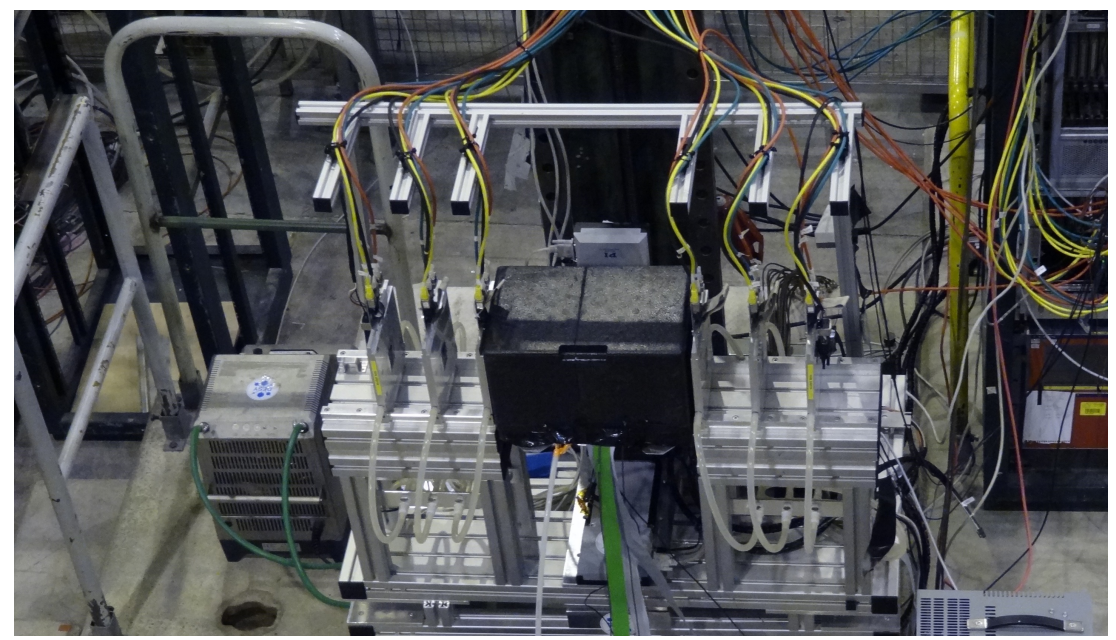

Figure 5.3: Typical IBL testbeam setup, having one telescope arm upstream and one telescope arm downstream. Between the telescope arms, the DUT cooling box is visible, which allows mounting of several DUTs inside, as well as space for dry ice. The cooling is necessary to keep the leakage current of the devices low, to avoid damages. Since the operation parameters of the IBL experiment specify a maximum operation temperature of $-15^{\circ} \mathrm{C}$, the dry ice cooling is a legitimate way to keep the devices within their design conditions.

following sections. The used DUT readout system, as well as the data streams of the telescope planes and the scintillators, have to be connected to the trigger logic unit (TLU) [60]. This central part of the data taking hardware is designed to distribute trigger signals to all connected devices. A trigger signal is sent if all four (depending on the settings the number can be lower) connected scintillators send a signal to the TLU. This process has to happen as fast as possible, since the interesting data otherwise could be shifted out of the DUT buffers. The typical time between receiving the scintillator signals and sending the trigger command is around $27 \mathrm{~ns}$.

The heart of the TLU is a commercial FPGA ${ }^{3}$ board. It allows to change the trigger settings via a USB connection, e.g. the AND mask of the scintillators, which execute a trigger signal. Beside that, the board allows to run the TLU in a so called "self trigger mode", where the data acquisition chain can be tested without beam. In this mode, the TLU sends trigger signals generated by the FPGA, ignoring the scintillator input. Up to six devices under test can be connected via Ethernet cables to each TLU, see figure 5.4. Here, two out of the eight wires in the cable are used for the trigger signals, which are transmitted in the LVDS$^{4}$ standard.

As soon as a trigger signal is sent to the connected devices, a BUSY signal is expected from each connected device. It stays active either as long as the data taking is active for a connected device or if the buffers of the data taking system are full. As long as the BUSY signal is active, no new trigger signal can be sent. In data taking mode, the TLU also distributes a trigger ID with each trigger signal. This ID is an integer number, which is increased by 1 , each time a new trigger signal is sent. In case of several data streams, which have to be mapped to each other, the trigger ID is extremely useful. In order to have the trigger ID in the data stream, the TLU sends the trigger ID through the trigger line to the connected devices, after receiving a designated signal. The BUSY signal stays high until this whole process is finished and the event is completely read out. This means that the time between two trigger IDs is at least as long as the longest readout time of the different connected devices.

\footnotetext{
${ }^{3}$ Field Programmable Gate Array

${ }^{4}$ Low Voltage Differential Signaling
} 


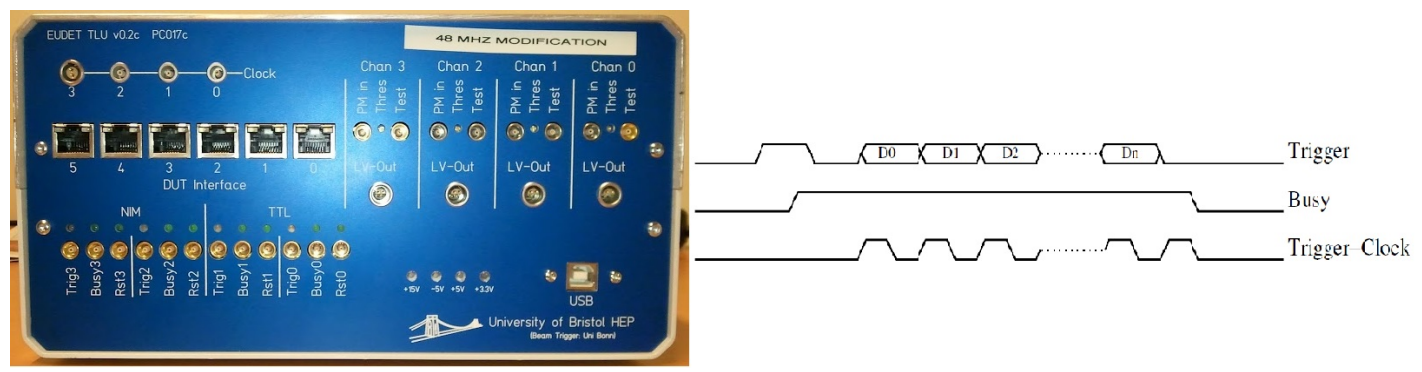

Figure 5.4: Left: A trigger logic unit (TLU) as it has been used in the ATLAS pixel testbeams. Up to six devices under test can be connected. Right: Data lines of a TLU, showing a trigger handshake, where data is sent from the TLU to the connected devices to synchronize the setup [60].

\section{USBpix Setup}

Figure 5.5 shows the USBpix board [61]. It is a modular system, which allows to connect different generations of ATLAS pixel modules by using the particular adapter cards. Compared to previous test systems, USBpix is not only highly modular, but also very compact. This system has been used as the default test system for IBL chip tests and sensor qualifications, providing several scan routines. An overview of the most important scans can be found in [40]. All digital functionality of the system is hosted in the FPGA of the Multi-IO board. In the FE-I3 configuration the scan routines are controlled by the on-board micro-controller. Beside that, the micro-controller hosts the communication between the FPGA and the USB port. The collected data is temporarily stored in an on-board SRAM. The on-board equipment allows to process the collected data, such that the connected PC receives well prepared data packages, which reduces the load on data lines. Another detail, that can be seen on the photograph of the Multi-IO board, is the RJ 45 (Ethernet) connector, which allows communication with the TLU. In testbeam data taking mode, the USBpix system receives trigger signals via this Ethernet connector and sends back the buffered hit information.

Communications with the different connected devices is handled by the adapter card. This card provides the communication between the connected device and the LVDS chip on the Multi-IO board, which generates the signals that are transmitted to the devices. The chip configuration is one of the main parameters that varies for each sample in the IBL testbeam periods.

Beside the modular hardware setup, the USBpix package also offers a modular software framework. This modularity opened the possibility to adapt the framework to several devices, like device emulators, probe station testing cards and thus also to future developments.

\section{RCE Setup}

In parallel to the USBpix system, another approach has been followed. At Stanford Linear Accelerator Center (SLAC) a test system has been developed, based on the Advanced Telecommunication Computing Architecture (ATCA) [63]. One of the main developments for this test system is the Reconfigurable Cluster Element (RCE), shown in figure 5.6. While the RCE system is a readout system candidate for future ATLAS pixel upgrade scenarios, up to now it has been used for testing of IBL staves and in testbeam campaigns. Due to the second fact, the system is explained in more detail in the following.

Figure 5.7 shows a schematic of a complete RCE setup.

The RCE boards are located in an ATCA crate, which is a state-of-the-art high speed bus system. It has been developed by different telecommunication companies and offers several advantages compared 


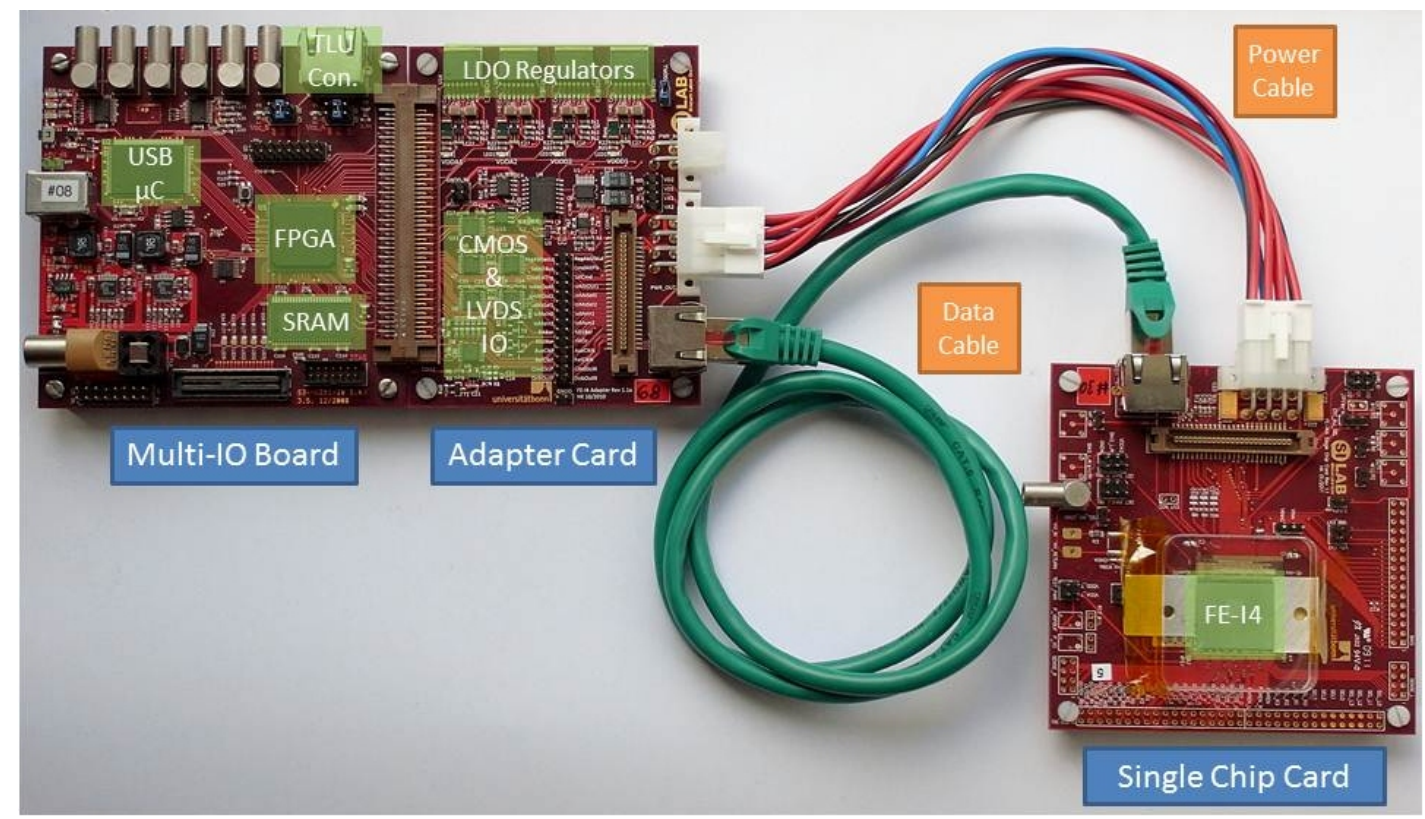

Figure 5.5: USBpix test system, consisting of a Multi-IO board, equipped with an FPGA and a micro controller, and an adapter card. Shown in this picture is a setup for FE-I4 devices [62].

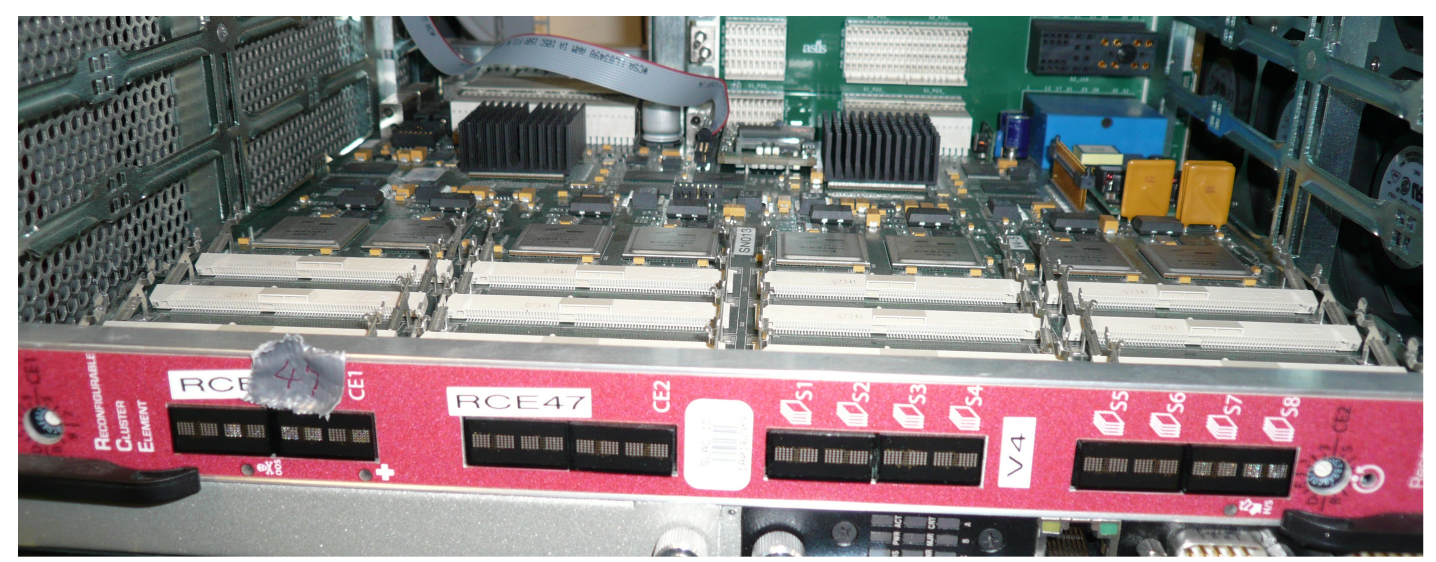

Figure 5.6: RCE test setup, as it is used for further developments of the RCE system. The same RCE setup was also used for device readout in the latest testbeam campaigns. The RCE processors are located below the black heat sinks.

to e.g. the $\mathrm{VME}^{5}$ standard, which has been used for previous readout systems. Not only the data handling speed has been increased compared to VME based systems, but also the reliability due to a hardware design, which is separated into several independent sub-structures. Since the RCE system is designed for a possible usage in future experiments, an optical component called Rear Transition Module (RTM) was developed. It allows to transmit data via optical fibres at a transmission speed of 3.125 GBit/s. An optical data transmission is important for data transmission between LHC detectors and counting rooms, which are typically on the order of $100 \mathrm{~m}$ apart. For lab tests, as well as for the testbeam setup, an electrical data transmission is available for easier handling. The RCE board itself is

\footnotetext{
${ }^{5}$ Versa Module Eurocard
} 


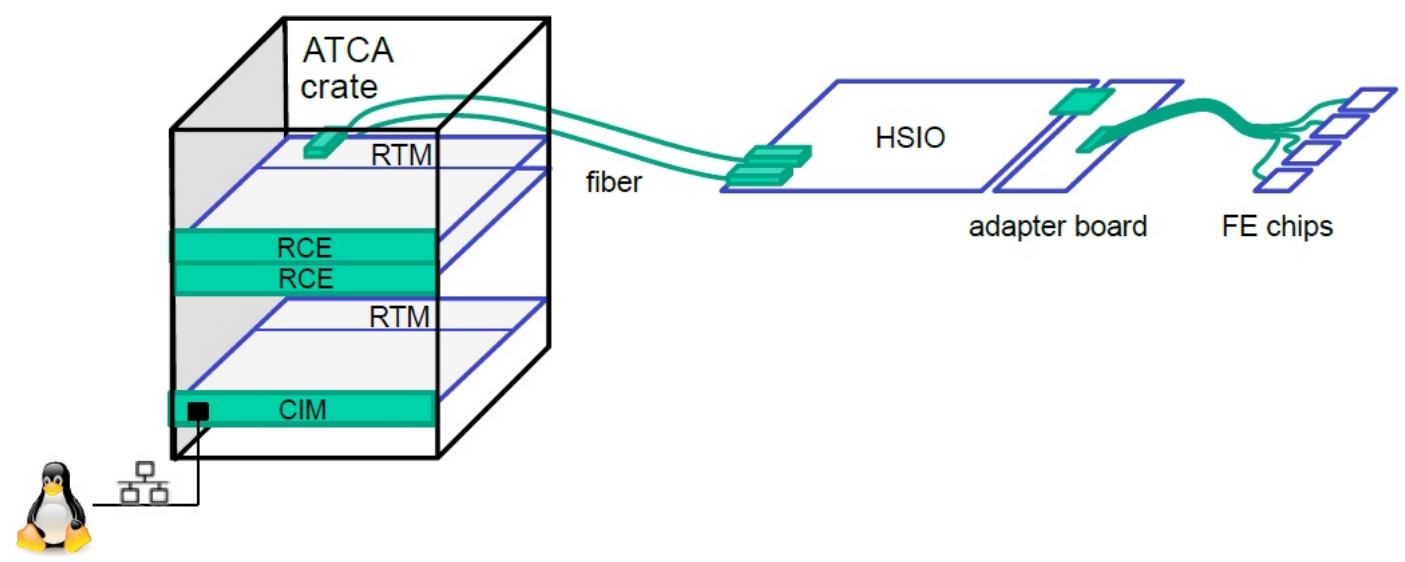

Figure 5.7: Schematic of a complete RCE setup, including optical data transmission and the usage of several RCE boards [63].

based on an FPGA of the Virtex 4 generation. It is designed to handle several ATLAS pixel modules in parallel, such that one RCE setup can be used for readout of all DUTs in a testbeam setup. The other parts mentioned in the schematic are the Cluster Interconnect Module (CIM), which provides an Ethernet connection to the user's PC and hence is designed to distribute the user commands to the readout system. Another part of the RCE readout system are the High Speed Input/Output Modules (HSIO) with connected adapter cards. The HSIO boards are the optical components on the detector side. In an LHC experiment setup, these cards would be placed close to the detector for transforming incoming optical signals to electrical and vice versa. Like in the previously mentioned USBpix setup, an adapter card, which is specifically designed for the connected detector modules, is used as interface between the main components of the RCE readout system and the detector.

\subsubsection{The EUDAQ Package}

Within EUDAQ ${ }^{6}$, data taking is organized in so called "producers". These producers are independent, modular software tools, that connect to each other via Ethernet connection, see figure 5.8. Here the central producers are the Run Control and the Data Collector. The Run Control is a graphical user interface, that allows the user to control the running processes, load different data taking configurations and start and stop runs. The Data Collector is designed to receive data streams from different producers, to merge these streams and finally write them to a raw data file on the hard disk. This producer creates a new file for each run that is taken. Each of the connected hardware devices has its own producer, e.g. telescope producer, ATLAS pixel producer, TLU producer. The latter one controls the trigger logic unit (TLU, see 5.1.3) and sends the timestamps to the data collector. As the name already suggests, the Log Collector collects logging information and displays it to the user. And finally, the Monitor is the online monitor within the EUDAQ package, to display live information on the data quality to the user (see next section). Furthermore, the EUDAQ package hosts the functionality of data conversion from raw-data files to $\mathrm{LCIO}^{7}$ format, which is the starting point of the track reconstruction, see 6.1.2.

To provide an easy way to add new detector types to data taking and data conversion, the data encoding part of EUDAQ is arranged using so called Converter Plugins [64]. Whenever a new detector type has to be added, a new converter plugin is sufficient to ensure proper data taking and data treatment

\footnotetext{
${ }^{6}$ EUdet Data AQuisition system

${ }^{7}$ Linear Collider I/O
} 
in the reconstruction steps. The basic part of a converter plugin is the specification of the data string that should be encoded, called RawDataEvent. Furthermore, the plugin extracts the (TLU) trigger ID, that has been assigned to the corresponding event and fills the extracted data into a Standard Plane, the EUDAQ internal data handling structure. Here an event means the time in which a sensor plane is active and records data, after receiving a trigger signal. A Standard Plane contains the charge information of the hit pixels of one sensor (if available). This information can be collected for several readout frames, in case a readout cycle is shorter than one event (e.g. for ATLAS pixel devices). All information collected for all planes in one event (active readout time of the sensors after trigger signal arrived), is stored in a Standard Event. Having this sorted information available, the conversion to LCIO format can be performed for each connected detector type. For more information, see [64].

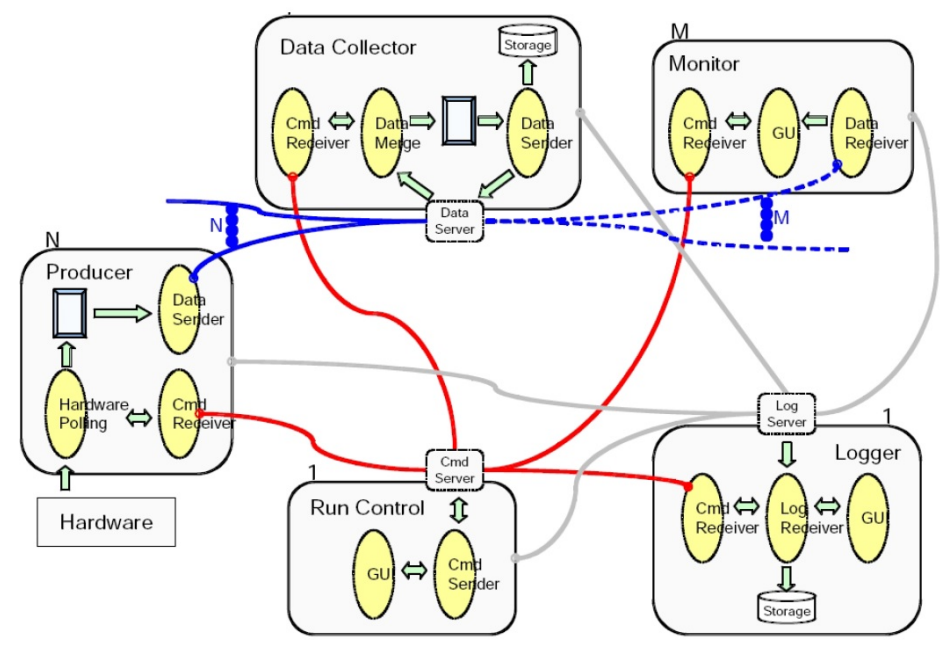

Figure 5.8: Schematic overview of the EUDAQ structure, grouped into the main producers. All producers are connected via Ethernet. For further details, see 5.1.4 [64].

\section{Online Monitor}

As part of the EUDAQ package, a live event display called "Online Monitor", is available. The Online Monitor either reads in the raw data file, that is written during data taking or can display preliminary information about runs that are already on the hard disk. During this online processing of data, a search for clustered hits is performed. In most cases an incident particle deposits charge in more than one pixel cell, thus, recorded hits that were caused by the same particle have to be combined to one cluster. The process takes a hit in a plane and searches its eight neighbours for further hits, until no more neighbours can be found in this matrix. Also a quick search for noisy pixels is performed, where all pixels that fired in more than $15 \%$ of, by default, 200 investigated events, are labelled as noisy or "hot". In figure 5.9 a screenshot of the Online Monitor is shown, indicating the number of clusters per event that are recorded on the second and third (plane IDs counting starts from 0) telescope plane. Both distributions peak at around 12-15 clusters per event, which is a good value for data taking. If the number of clusters per event reaches values above $\sim 30$, the correct assignment of clusters to the corresponding tracks is not possible anymore, since several clusters compete for the same track.

After installing the testbeam setup, it is usually quite hard to get a feeling for the alignment of the planes. Since the telescope planes are mounted on a solid, well-aligned structure, their misalignment with respect to each other is small. The DUTs are usually mounted as central as possible, using by- 


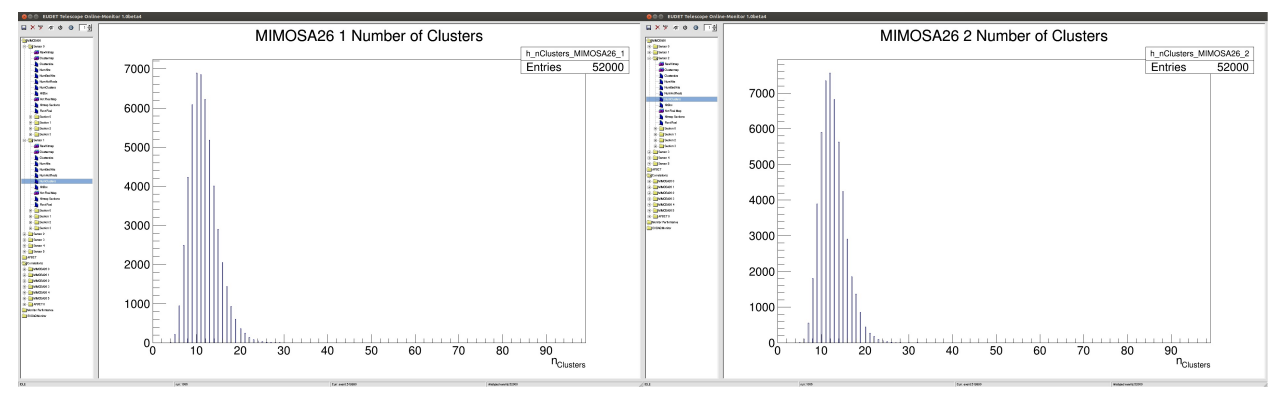

Figure 5.9: Online Monitor screenshot, showing the number of clusters for the second and third telescope plane during data taking. These plots give a first live impression of the data quality.

eye-alignment on an X-/Y-stage. To get a better idea of the DUT positions, the Online Monitor provides cluster correlation plots. These plots provide a useful hint of the plane positions, since the clusters along a particle track are spatially correlated between the sensor planes in the setup. Consequently, correlation plots are produced, showing the correlations separately for $\mathrm{x}$ - and y-direction. The information is filled into 2D histograms, where the reference information is always the first telescope plane, "Mimosa26 0". An example is shown in figure 5.10. In a perfectly aligned setup without noise hits, the correlation plots would just show a diagonal line from the lower left to the upper right. Each incident particle would hit the sensor planes at the same $\mathrm{x}$ - and $\mathrm{y}$-coordinate. In figure 5.10 several typical effects are visible. First, the slope of the $\mathrm{x}$ - and y-correlation of the first telescope plane (Mimosa26 0) and the first DUT (APIXCT 0), shown in the top left of the figure, is less than 1. Reasons for this can be on the one hand, the larger pixel pitches of the APIX devices, compared to Mimosa26 sensors and on the other hand, a probably not perfectly parallel mounting of the planes. Furthermore, the number of entries in the histogram is quite small, compared to the other histograms. This can be explained by the longer integration time of the telescope planes and fewer detected hits, especially in the first DUT, which can be also seen in figure 5.11. The x-correlation histogram of Mimosa26 0 and APIXCT 1 (third histogram in figure 5.10) shows a falling correlation band, which indicates that the orientation of the planes was different.

The correlation histograms of the telescope planes show some more effects. With increasing plane ID, which means a position further downstream, the correlation bands get broader. The shown histograms are taken from a DESY testbeam campaign, where the beam energy of $4 \mathrm{GeV}$ is low enough to result in a significant amount of multiple scattering. Thus, the correlated $x$ - and y-positions of clusters are shifted more and more from the ideal correlation band. Finally in all histograms, showing the x-correlations, vertical and horizontal lines are visible. These are caused by noisy pixels, which constantly give a signal and therefore show an uncorrelated behaviour with respect to the hits of the other plane. During data taking, these pixels are identified and masked.

Finally, the Online Monitor also displays information about the DUTs without any tracking, just to allow a check of the data quality during data taking. An example can be seen in figure 5.12. This device overview provides a lot of information about the DUT (APIXCT 1) performance. Starting from the upper left, they are the following:

- TOT Clusters shows the charge induced by the incident particles. Here the charge that has been deposited in clusters is displayed, since the total charge induced by a particle is of interest. This information is given in units of time over threshold (ToT, see section 3.2.3). The peak position of this distribution depends on the tuning of the device. Even without any tracking information the distribution already roughly shows the expected shape of a Gaussian distribution, folded with a 

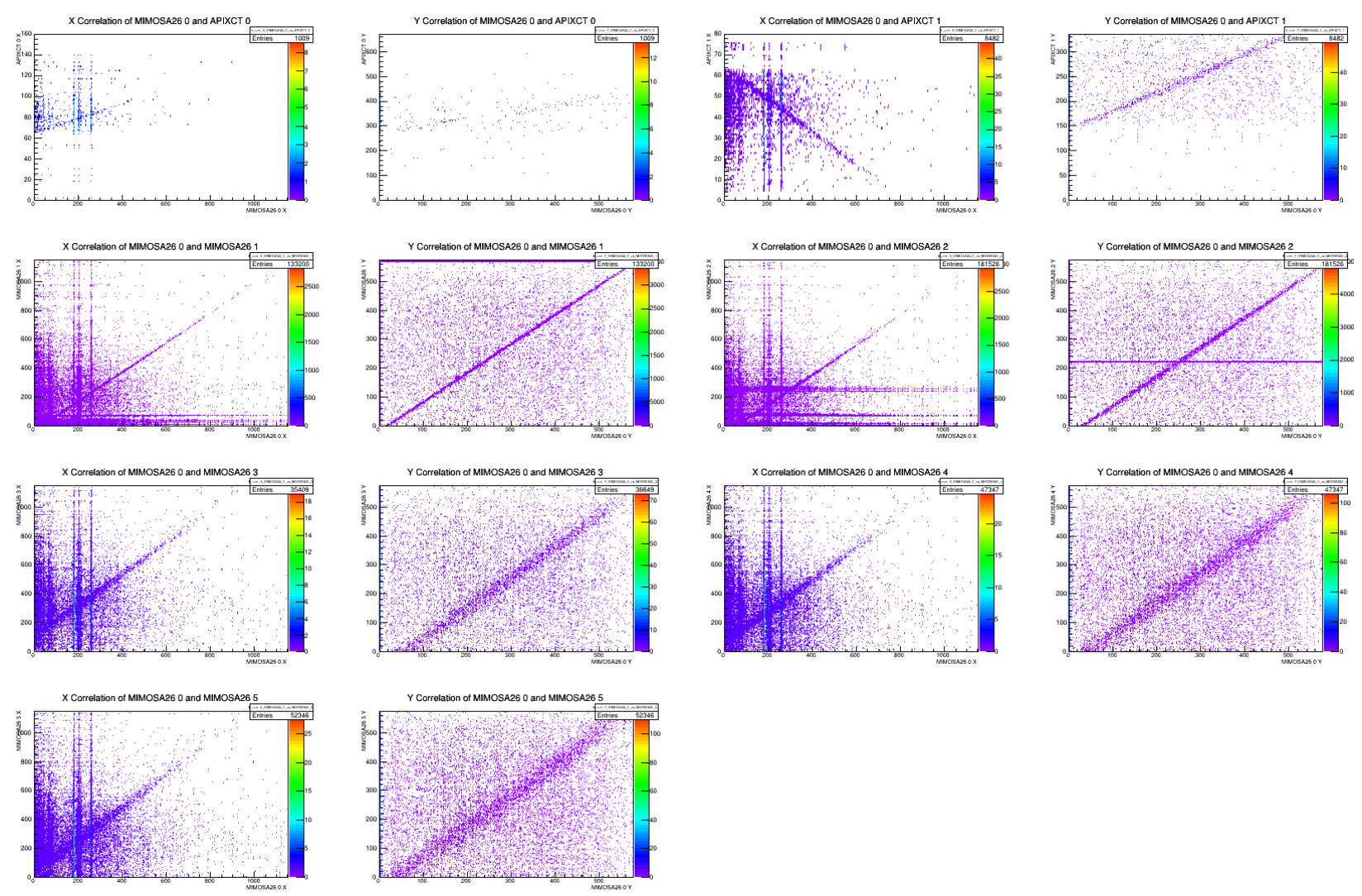

Figure 5.10: Hit correlations between all devices in the used setup with respect to the first telescope plane. The Online Monitor correlation plots do not take into account any information that is provided in the geometry description file, but still they can give a good estimate for the alignment of the setup. Note: the Online Monitor screenshots were taken during a testbeam campaign of the planar pixel sensors community in November 2013.

Landau distribution.

- Clustersize in $\mathbf{X}$ gives the calculated cluster size in $\mathbf{X}$-direction, which is the $(250 \mu \mathrm{m})$ long pixel direction. Since the device is not rotated with respect to any axis, small cluster sizes are dominant, with a background of large cluster sizes in the distribution. This background vanishes when the tracking (chapter 6.1) is applied.

- Clustersize in $\mathbf{Y}$ is basically the same as the previous point, just in the $(50 \mu \mathrm{m})$ short pixel direction.

- Cluster Hitmap gives the position of clusters on the device, in coordinates of columns (X) and rows (Y). Around column 60 and row 150 the projected edges of the trigger scintillators are visible. Hits outside of the region covered by the scintillators are either real hits or noise, detected while the sensor is active due to a real hit within the scintillator.

- Clustersize is a histogram, where the total cluster size ( $\mathrm{x}$ - and $\mathrm{y}$-direction multiplied) is shown.

- Hitoccupancy shows the occupancy distribution of the sensor pixels. In this case, the occupancy of the pixels was low, such that the entries in the histogram merge with the y-axis. 

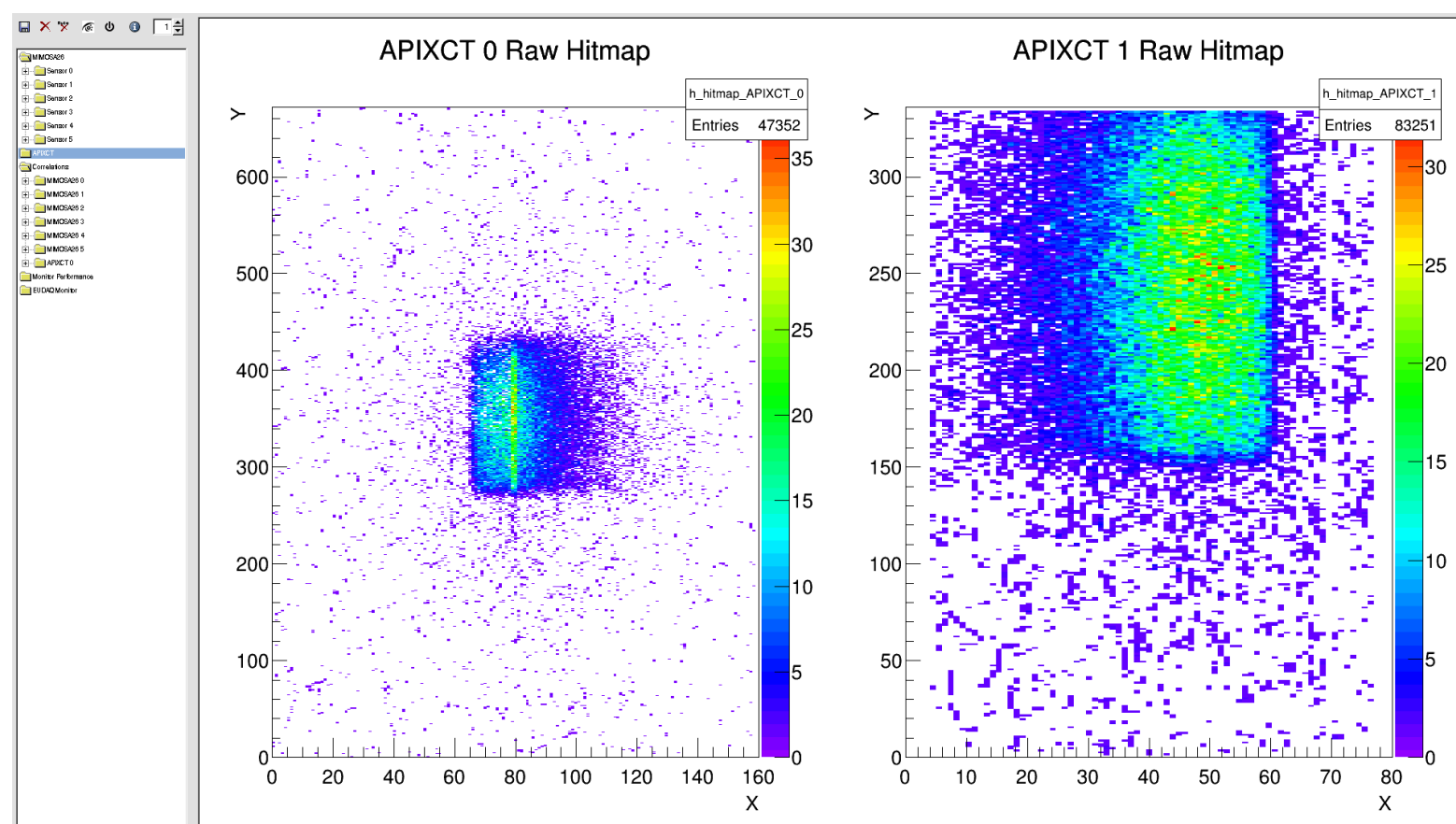

Figure 5.11: Hitmaps for the two devices under test. On the left side the position in coordinates of $\mathrm{X} / \mathrm{Y}$ in units of pixels are shown for a 4-chip module, on the right side the same information is provided for a single chip module. In both cases the crossing area of the trigger scintillators is clearly visible.

- Hot Pixel Map is a 2D map, showing the position of pixels, identified as hot/noisy pixels. In this example no pixel is identified as a hot pixel yet. The position is given in column/row coordinates.

- LVL1 Cluster Distribution shows the LVL1 timestamp of clusters. This timestamp is a FrontEnd chip internal counter in units of $25 \mathrm{~ns}$, which indicates the time between an arriving trigger signal and a hit being read out. The position of the peak depends on the tuning of the device.

- LVL1 Pixel Distribution is a histogram showing the LVL1 timestamp of the single pixels. This histogram has more entries than the previous one, since the value for each single hit is displayed.

- LVL1 Clusterwidth shows the differences of the earliest and the latest hits within clusters in values of LVL1s. It is a measure of the timewalk.

- Number of Invalid Hits gives an overview of how many hits per event are invalid, due to e.g. wrong data word formats.

- Number of Clusters is an illustration of the cluster multiplicity, which means how many clusters are found per event. This number is an important data quality check, since a high cluster multiplicity means a high number of combinatorial possibilities to assign clusters to a track candidate. Thus, very high cluster multiplicities reduce the data quality.

- Number of Hits displays the hit multiplicity per plane, which is the number of hits per event in a device.

- Number of Hot Pixels gives an overview of how many noisy pixels are found per device. The occupancy limit can be set by the user. 
- Raw Hitmap shows all hits that have been registered in the device, without applying a clustering algorithm or any cuts. Again the shape of the crossed scintillators is visible in the upper half of the histogram.

- TOT Single Pixels again shows the charge induced by the incident particles. The difference compared to the TOT Clusters histogram is that here ToT values for the single hits are displayed. This means that especially no clustering and no noisy pixel algorithm is applied yet. The maximum ToT value that can occur here is 14 for FE-I4 devices. The peak is around the expected tuning value of 7 , surrounded by a floor of early, respectively late hits, which are mostly noise hits.

\subsection{Testbeam Facilities}

Finally, when the whole setup is complete, it can be brought to a beam facility for device testing. For the case of the IBL experiment, all testbeam campaigns took place in the testbeam facilities of CERN and DESY. An overview of these two sites is given in the following.

\subsubsection{DESY}

The German accelerator and particle physics research centre Deutsches Elektronen Synchrotron (DESY) is located in Hamburg. It was founded in 1959 and hosted many famous accelerators and experiments, like $\mathrm{H} 1$ and ZEUS, which operated until the shutdown in 2007.

Figure 5.13 shows the electron $\left(\mathrm{e}^{-}\right)$and positron $\left(\mathrm{e}^{+}\right)$production process at DESY $[65,66]$. In the DESY II accelerator either positrons or electrons are accelerated. For the testbeam lines, the accelerated particle beam is lead onto a $7 \mu \mathrm{m}$ carbon fibre, where photons are produced via bremsstrahlung processes. Further down along the beam direction, a metal plate functions as second target and converts the photons back to electron/positron pairs. With the used dipole magnet, electrons and positrons are being spread out to a horizontal fan, where the particles are separated in terms of charge sign and energy. The energy of the electrons/positrons arriving in the testbeam line can be varied between $1 \mathrm{GeV}$ and $6 \mathrm{GeV}$ by the users using this dipole. Finally, a collimator shapes the beam for testbeam measurements. The achieved particle rate depends on the selected beam energy, as can be seen in figure 5.14. At DESY testbeam lines a continuous beam is delivered to the users. Due to the maximum possible beam energy of $6 \mathrm{GeV}$, multiple scattering effects play a significant role, which allows typically a maximum of two DUTs per setup. Otherwise the amount of material in the beam becomes so large, that tracking becomes excessively complicated.

\subsubsection{CERN SPS}

Located down to 50 meters underground at the European Organization for Nuclear Research CERN, the second testbeam facility that has been used by the IBL community is the Super Proton Synchrotron (SPS). As already described in section 2.1.1, the SPS is the final accelerator in the pre-accelerator chain of the Large Hadron Collider. It accelerates protons to a beam energy of $450 \mathrm{GeV}$. Beside providing protons for the Large Hadron Collider, the SPS also provides particles for several testbeam lines and other experiments. These are located at the "North Area" of CERN, in Prévessin-Moëns (France). All testbeam lines in the SPS North Area can provide different kinds of particles with a certain energy range. This is achieved due to three main beam targets, called T2, T4 and T6, which are hit by the SPS proton beam. Depending on the kind and orientation of the used target, the secondary beam usually consists of hadrons and electrons, where the electrons are stopped using a thin lead absorber. The particle energy 
can be varied between around $20 \mathrm{GeV}$ and $180 \mathrm{GeV}$. Further down along the beam direction, a large variety of targets and absorbers can be used in combination with the primary target settings, to deliver the desired kind of particle for the particular user, see figure 5.15. So called "wobbling stations" allow this tuning of the beam properties.

Since the beamlines for testbeam users are not the only users of the SPS beam, a beam spill structure has been set up. Due to the spill structure, the beamlines for testbeams in the North Area receive particles for around ten seconds per cycle (which is about $40 \mathrm{~s} \mathrm{long}$ ), compared to the continuous beam at DESY. During one spill, typically a maximum number of around 4500 triggers can be recorded with the IBL testbeam setup. The typical beam energy during IBL testbeams has been $120 \mathrm{GeV}$, which has a significant advantage in terms of multiple scattering, compared to the $6 \mathrm{GeV}$ beam at DESY. This allows not only a better resolution for in-pixel studies, but also makes the data reconstruction significantly easier. 

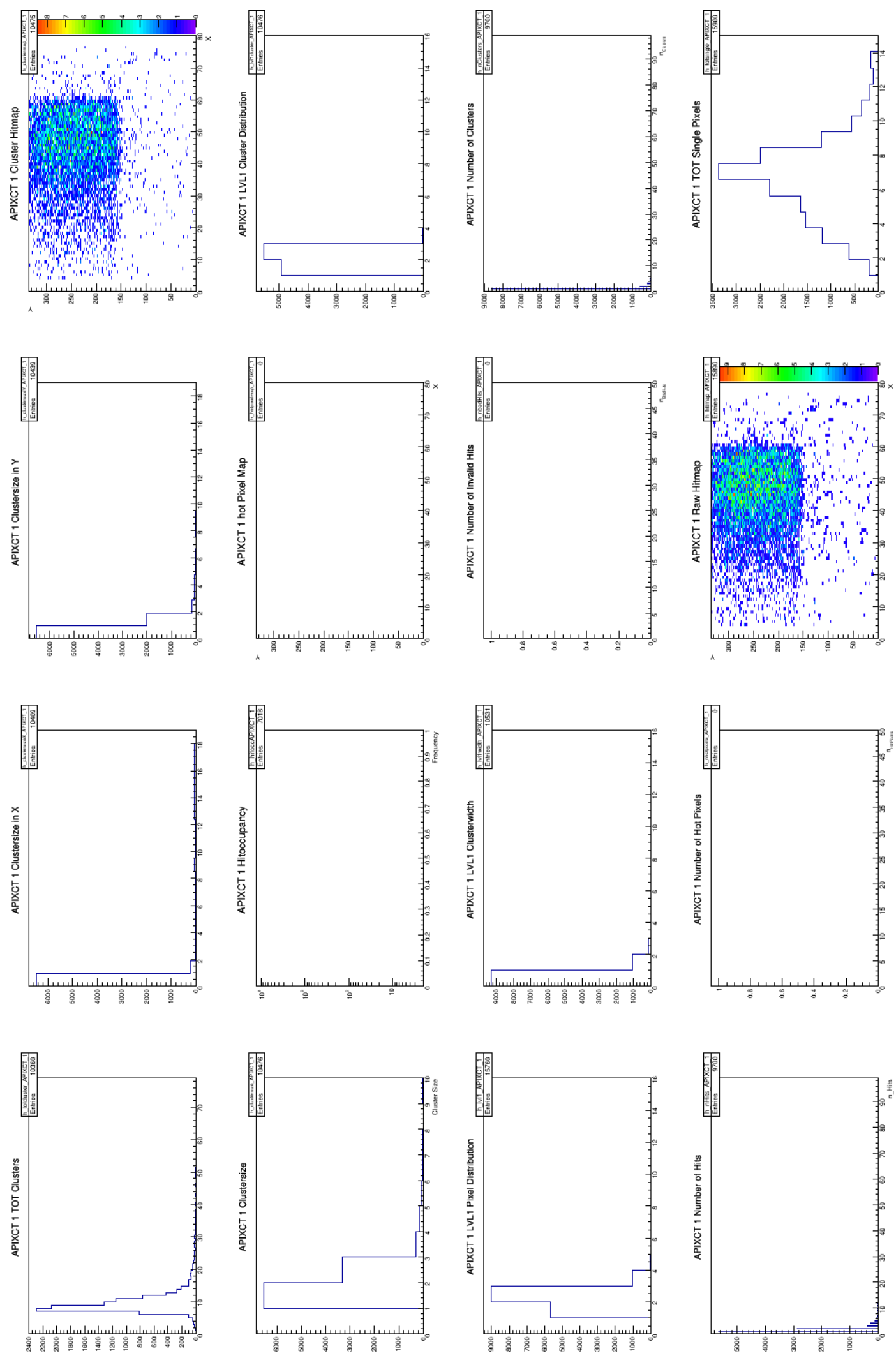

Figure 5.12: Screenshot of all information the Online Monitor provides for a device under test. The considered device is a single chip module. Since the histograms are being filled during data taking and the necessary calculations for the different kinds of histograms need different computing power, not all histograms are filled in the same speed. 


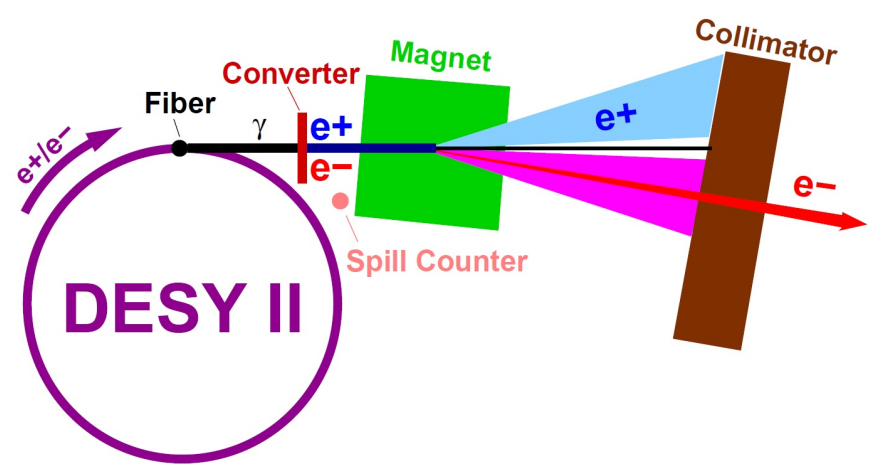

Figure 5.13: Schematic layout of the electron/positron production at DESY for testbeam users. The shown dipole magnet is used for spreading the particle beam into a horizontal fan, such that particles are separated in terms of charge sign and energy [65].

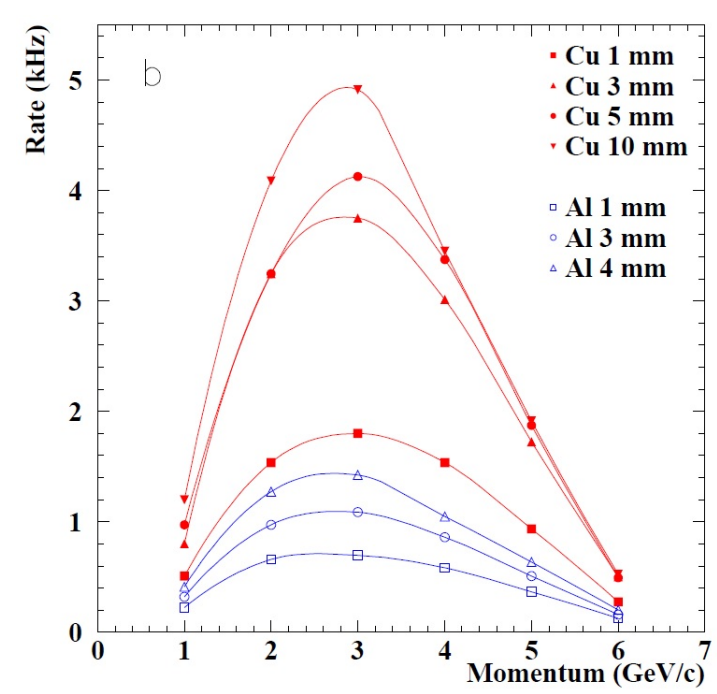

Figure 5.14: Particle rates at DESY testbeam, depending on the chosen energy and the secondary beam target. The particle energy in the beamline can be varied by the users between $1 \mathrm{GeV}$ and $6 \mathrm{GeV}$ [66].

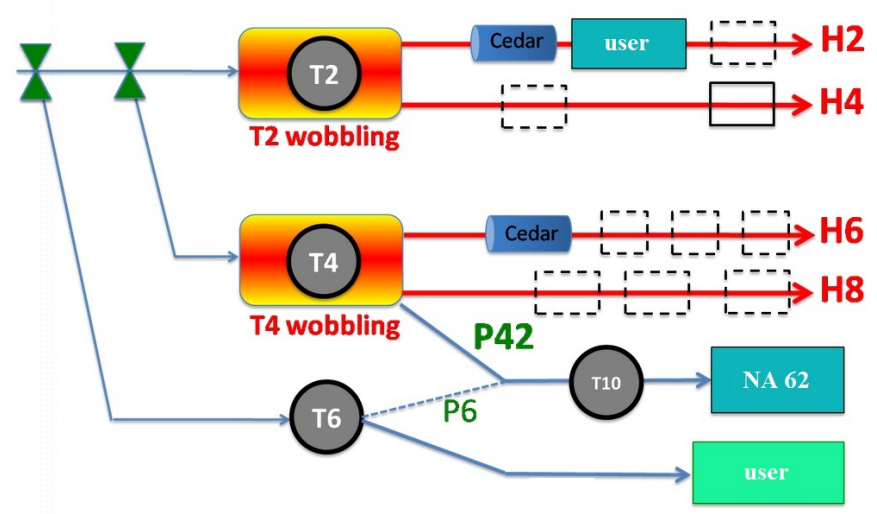

Figure 5.15: Schematic overview of the CERN North Area beamlines, including the primary targets and wobbling stations, which allow users to choose between a variety of particles and particle energies (taken from CERN NA62 Collaboration website; at time of writing http://na62.web.cern.ch/na62/ExperimentalArea/SchematicNorthArea.html). 



\section{Chapter 6}

\section{Testbeam Track Reconstruction and Analysis Tools}

\subsection{Track Reconstruction - The ILC Framework}

For track reconstruction, a software package was used, which has its origins in the ILC ${ }^{1}$ soft framework. The framework included the basic packages, especially EUTelescope which will be described in the following. Within the last years the provided software has been extended and adapted in almost all features, to match the requirements of ATLAS pixel testbeam data reconstruction and analysis. In chapter 6.1.2, the current status of the software, the processes behind, the different options and extensions and further details about the track reconstruction for ATLAS pixel devices will be described.

\subsubsection{Marlin}

Applications that are meant to be run within the ILC framework, can be written in a modular structure [67]. The single modules are called MARLIN ${ }^{2}$ processors. For data treatment and analysis, the used data format for Marlin processes has been fixed to the $\mathrm{LCIO}^{3}$ format. Here LCIO has the function of being a persistency framework, as well as a data model for the ILCsoft framework. This format is also used for current ATLAS pixel testbeam reconstruction, providing both, a data model including hits, clusters, etc. and the general file format to store the processed data. Since the LCIO format was designed to handle full events of ILC data, but also provides the possibility to extend the number and types of stored information, it was extended to fulfil all requirements of ATLAS pixel data. Thus, the whole track reconstruction chain works based on the LCIO data format.

\subsubsection{EUTelescope}

EUTelescope [68] is the software package which contains all track reconstruction steps needed for ATLAS pixel testbeams and other device types. The general software skeleton of this framework is provided and maintained by the EUDET group ${ }^{4}$. Meanwhile several copies of the EUDET telescope, some of them with slight modifications, exist. The EUTelescope package is able to treat data of all EUDET-like telescopes and thus this package has been chosen as the default and only reconstruction tool for all ATLAS pixel testbeams. The different processors that are used for ATLAS pixel testbeam reconstruction are described in the following. All of the used reconstruction steps are organized as Marlin processors, partly interacting with external tools. In figure 6.1 the steps from raw-data files to final track files are shown, to give an overview for the following sub-chapters.

\footnotetext{
${ }^{1}$ International Linear Collider

${ }^{2}$ Modular Analysis and Reconstruction for the LINear collider

${ }^{3}$ Linear Collider I/O

${ }^{4}$ www.eudet.org; see chapter 5.1
} 


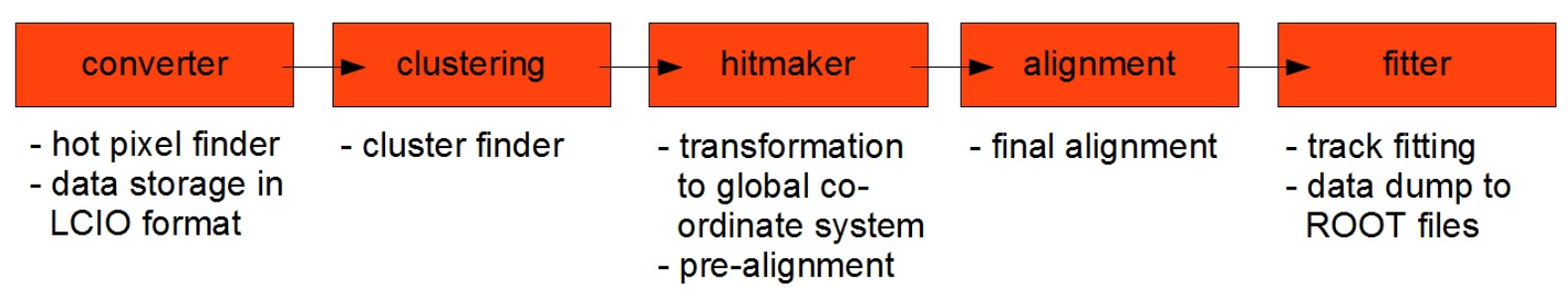

Figure 6.1: Track reconstruction chain, as used for ATLAS pixel testbeams. The raw data is read in and after five processing steps, a ROOT file containing all relevant track and hit information for further analysis is generated.

\section{The GEAR Library}

The first step towards reconstructing a particle track through a testbeam setup is a proper description of the used setup. Within the EUTelescope package the ILC library GEAR ${ }^{5}$ is used. GEAR uses XML ${ }^{6}$ files to describe geometrical properties of the individual devices used, as well as the absolute position and rotations of each sensor in the global coordinate system of the setup. While the GEAR library has been developed to describe whole detector systems, especially the ILC experiments, special classes are provided to describe arrangements with a large number of relatively small sensitive areas arranged behind each other, e.g. testbeam setups.

Taking the geometry information provided in the XML file, a testbeam setup can be described completely. Furthermore, in a later step of the reconstruction chain this information is used to construct a 3D space point for each sensor hit in the setup, as well as to transform all coordinates into a global Euclidean coordinate system. The most important values that can be given in the geometry description file are pixel pitches in $\mathrm{x}$ and $\mathrm{y}$ direction, relative $\mathrm{x}, \mathrm{y}$ and $\mathrm{z}$ position of each device, and rotations. Here, the latter are special in the sense that rotations can be applied in two different ways. On the one hand, each device can be rotated around the beam axis (by convention z-axis) using the rotation matrix:

$$
\left(\begin{array}{l}
x \\
y
\end{array}\right)=\left(\begin{array}{cc}
\cos \alpha & -\sin \alpha \\
\sin \alpha & \cos \alpha
\end{array}\right)\left(\begin{array}{l}
x_{0} \\
y_{0}
\end{array}\right)
$$

Here $x_{0}$ and $y_{0}$ are the coordinates in the local sensor coordinate system and $\alpha$ the applied angle. Since this matrix is typically used to apply rotations in steps of $\alpha=n \cdot 90^{\circ}$ for a rough specification of the device orientation, the four matrix parameters are combinations of the values $-1,0$, and 1 . An example is shown in figure 6.2.

On top of that, rotations can be applied as rotation angles in all possible directions. This especially becomes an important issue for measurements where sensors have been tilted on purpose to a given angle. The two staged way of applying rotations makes the setup description quite simple, since the user can just change the value of one rotation angle after rotating a device, instead of calculating matrices.

\section{Data Conversion and Re-Synchronization}

As a first step of the track reconstruction, the raw data files are converted to LCIO format (see section 6.1.1). During this conversion, the hit informations of the telescope planes and the devices under test (DUT) are written into separate data collections. The separation of telescope related information and DUT information in different data collections is realized for all steps of the reconstruction chain. Parts of the data converter need a similar functionality as the online monitor (see chapter 5.1.4) already

\footnotetext{
${ }^{5}$ GEometry Api for Reconstruction

${ }^{6}$ eXtensible Markup Language
} 


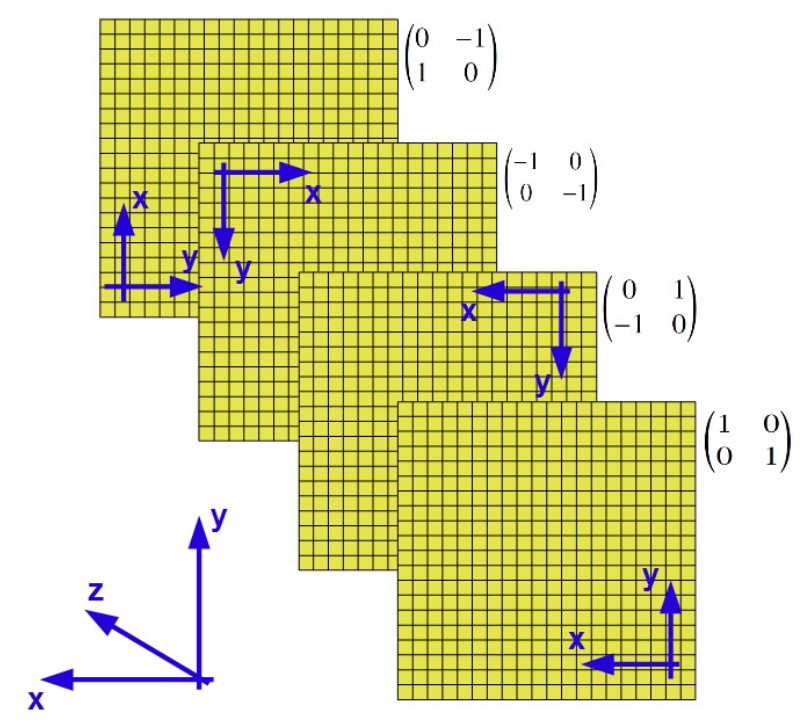

Figure 6.2: Example of possible coordinate system rotations in the GEAR library, using rotation matrices.

requires, therefore EUTelescope is linked to EUDAQ (see section 5.1.4). Especially the same data converter plugin as for the online monitor is also needed for this first step of EUTelescope, where the plugin in both cases performs the bit stream conversion. The data is converted to LCIO format and finally saved to files using the EUTelOutputProcessor. The produced collections are further used in this processing step to check the synchronization of data events.

With each trigger signal, and thus with each readout cycle of the system, the TLU (chapter 5.1.3) increases the event ID by 1 and this ID is written into the outgoing data streams. The streams for the telescope data and the DUT data are still separate at this point, thus caused by hardware incompatibilities or software problems, wrong and especially a different TLU trigger IDs could be written into the data streams, see figure 6.3. During the converter step, the TLU IDs of the different streams are compared event by event to the expected ID and in case of an unexpected TLU ID shift, the incorrectly assigned TLU IDs can be corrected. In summary, the events get re-synchronized in this way. The correct assignment of TLU IDs to their corresponding events becomes especially important in the later steps of the reconstruction, where e.g. correlations between different planes are calculated.

\section{Identification of noisy pixels}

Beside the features mentioned above, another important task of the data conversion step is the search for noisy pixels. Finding and removing noisy pixels is important to avoid fake tracks in the subsequent alignment and fitter steps. The alignment quality would suffer severely in case a pixel is noisy and therefore fires in each event. These noise hits could be taken into account as a track match candidate for the tracks, used in the alignment step. Thus, the alignment would be biased by the influence of noise hits. The same argument would be a problem for the final track fitting, where too many fake tracks caused by one device would even lead to wrong analysis results for the other devices under test. To fulfil the task of masking noisy pixels, first an occupancy measurement is performed. The occupancy measurements and the subsequent determination of the noise masks are performed in the same way for telescope planes and DUT planes, where individual masks for each device are created. For the occupancy measurement the pixel hit probability per event is calculated, taking into account the first 10,000 events of the processed 
file. The calculated values are stored in a database file, using separate collections for the telescope data and DUT data. In a second step of noisy pixel filtering, the created database file is opened and an occupancy cut is applied, where the default value is $10^{-3}$. All pixels exceeding this value are then written into another collection of the $\mathrm{db}$ file and can be masked in the later reconstruction steps. For ideal conditions, a noise occupancy measurement should be performed without beam, since all pixels exceeding the occupancy cut under these conditions are noisy without any ambiguities. The database file containing the masked pixels of such an off-beam run, can be applied for all runs taken with the same setup.

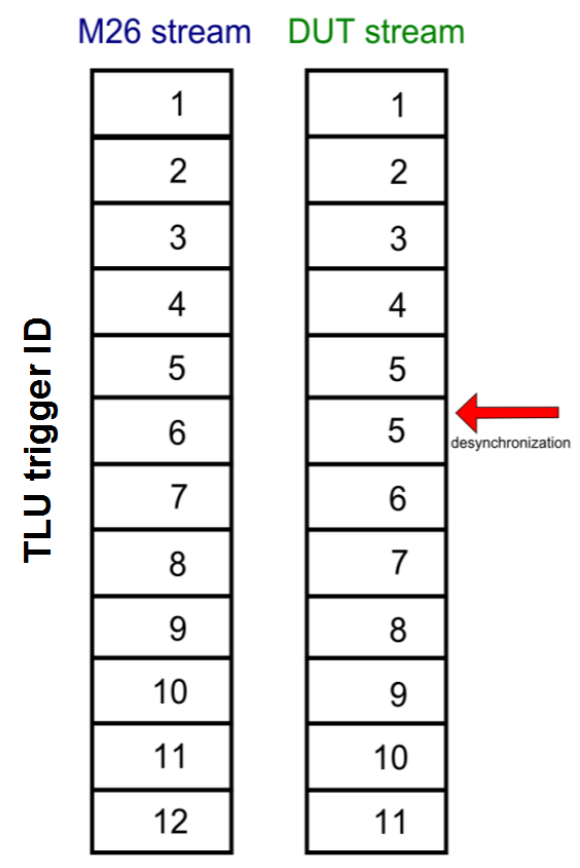

Figure 6.3: With each trigger signal, the trigger IDs written into the data streams are increased by 1 . In case of hardware incompatibilities or software problems, different TLU trigger IDs could be written into the data streams of the telescope and the ATLAS pixel DUT, resulting in desynchronized data streams.

\section{Clustering}

After the hit data is converted to LCIO format and the hot pixels are stored in a db file, an event based search for clusters is executed. Here a cluster consists of a number of hits in one plane, which were caused by the same incident particle. Typically a single particle deposits charge in 1-2 pixel cells, assuming non-rotated sensor planes. For highly rotated planes, the clustersize can increase up to the number of pixels the device is equipped with in beam direction. The clustering is done separately for the telescope planes and for the ATLAS pixel DUTs (APIXclustering).

For telescope clustering mainly two algorithms are used. The simplest amongst them is a fixed-frame clustering. In that algorithm, plane by plane a search for hits is executed. As soon as a hit is found, the neighbouring pixels are checked for hits. The size of the window in which further hits are being searched for can be set in a steering file, e.g. a $3 \times 3$ window. The fixed-frame clustering is the default method of non-zero-suppressed telescope data streams, which are produced by older generation telescopes. The fact that the size of the hit search frame has to be fixed, gives the name to this kind of 
algorithm. During data taking with the M26 telescope planes, zero suppressed data streams are written. Thus, a more advanced clustering method is used, which is called "sparse clustering". This method is optimized for cluster finding, when having a list of hits for each event (which is the definition of zero suppressed data), instead of a data set including information about each pixel for each event (non zero suppressed). The method does not require fixed sizes of hit search frames or any other restrictions. The algorithm first sorts all hits in an event according to their position on the sensor, as well as to the ToT signals of the single hits, in a list ${ }^{7}$. Here the hotpixel db file is used to mask all noisy pixels in the telescope planes. Afterwards for the sorted hits in the list, a search for neighbouring hits in the eight adjacent pixels is performed, as can be seen in figure 6.4. First, the three pixels in the row below the

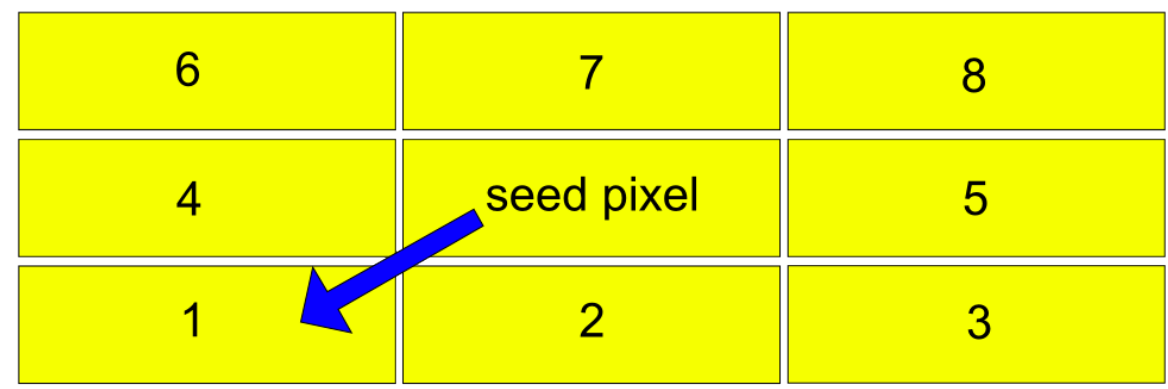

Figure 6.4: The sparse clustering algorithm performs a search for neighbouring hits, starting from the seed pixel. In a first step cells 1 to 3 are checked, afterwards 4 and 5 and finally cells 6 to 8 . More details on the functionality can be found in the text.

seed pixel are checked for hits, afterwards the ones left and right and finally the three pixels above the seed pixel. All hits found in this search range are added to a cluster group. As soon as this search is finished, the algorithm continues the search for further neighbours for each hit in the cluster group until no more neighbouring hits can be found in the same event. To properly separate multiple clusters in the same event, a minimum distance between two clusters (in units of pixel size) can be defined, as well as a threshold for defining the seed pixel. In case of the M26 data the latter one is unused, since the readout is binary and zero-suppressed.

The cluster search for ATLAS pixel devices is done with a custom processor. The APIX clustering processor is mostly based on the previously described sparse clustering, but provides some small extensions. One detail that has to be mentioned compared to the telescope clustering is that in the DUT clustering the hot pixel mask is not used, to not loose any hit information. Listing of the hits is done in the same way as in the standard sparse clustering, but the search for neighbours is adapted to the fact the APIX pixels are rectangular, while M26 pixels are squares. Thus, the APIX clustering allows to specify a minimum cluster distance separately in $\mathrm{x}$ - and y-direction, a minimum diagonal distance and a minimum time distance in units of LVL1 triggers. For data reduction of very noisy sensors, minimum and maximum cluster sizes can be defined. In case a DUT is highly rotated, this cut can be useful to suppress noise hits, which are typically single hits. Thus, for highly rotated devices, all 1-hit clusters can be excluded. To find out which pixel hits were recorded in neighbouring pixels, first each hit in an event gets assigned an incremented number (see figure 6.5). Afterwards each possible combination of two hits is checked and gets assigned an identifier in the format (A, B). Now the clustering algorithm checks, whether pixels combined as such an identifier pair are neighbours, for example the pair $(1,2)$ in the shown example, or no neighbours, e.g. the pair $(1,6)$. By assigning temporary flags to the hit pairs,

\footnotetext{
${ }^{7}$ M26 sensors have a binary readout, thus the ToT value of each pixel is 1, while APIX devices provide charge information.
} 


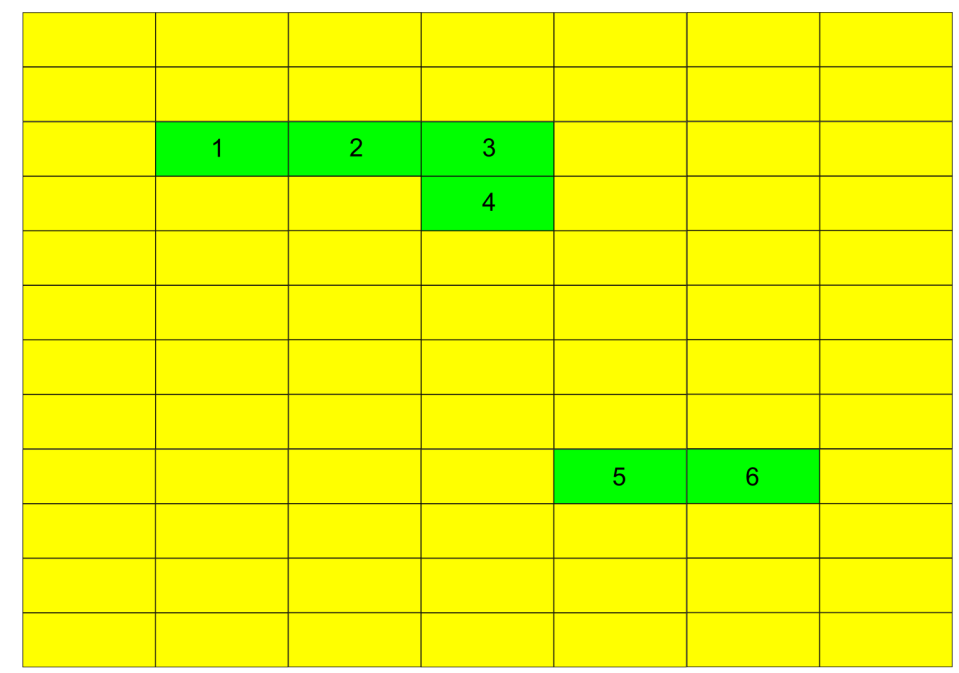

Figure 6.5: Example of a 4-hit cluster and a 2-hit cluster in one event. Each hit in the event gets assigned a number for further processing in the cluster finding algorithm.

clusters are identified sorted event based into a cluster collection.

Afterwards either the analogue (taking into account the ToT value of each participating pixel) or digital center of gravity can be calculated for each cluster, depending on the clustering settings. The difference between the digital and analogue center of gravity can be seen in figure 6.6. Finally, the cluster IDs, cluster sizes and center of gravity shifts in $\mathrm{x}$ - and y-direction are written to the clustering LCIO file. The processor also produces several control plots as output. Amongst the most important ones are the
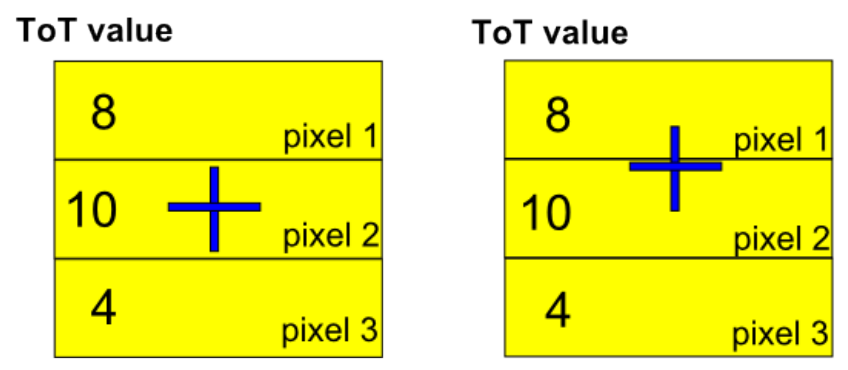

Figure 6.6: Left: the digital center of gravity algorithm sets the cluster center to the geometrical center of the cluster. Right: the analogue center of gravity weights the position calculation with the ToT value. Thus, the cluster position is shifted towards the pixel with higher charge.

cluster sizes per run, which should be around two for not rotated devices and higher with increasing rotation angle. The second important number is the cluster multiplicity (which is defined as the number of clusters per event). This value gives an indication on the beam quality, as well as the sample tuning quality. Very noisy samples or extremely high-rate beams will lead to a high cluster multiplicity. If this value is too high, it will lead to alignment and track fitting problems, due to too many ambiguous track candidates. Finally, the cluster ToTs are shown, that should correspond to the tuning of the device. Examples for these control plots can be seen in figure 6.7 and 6.8 . 

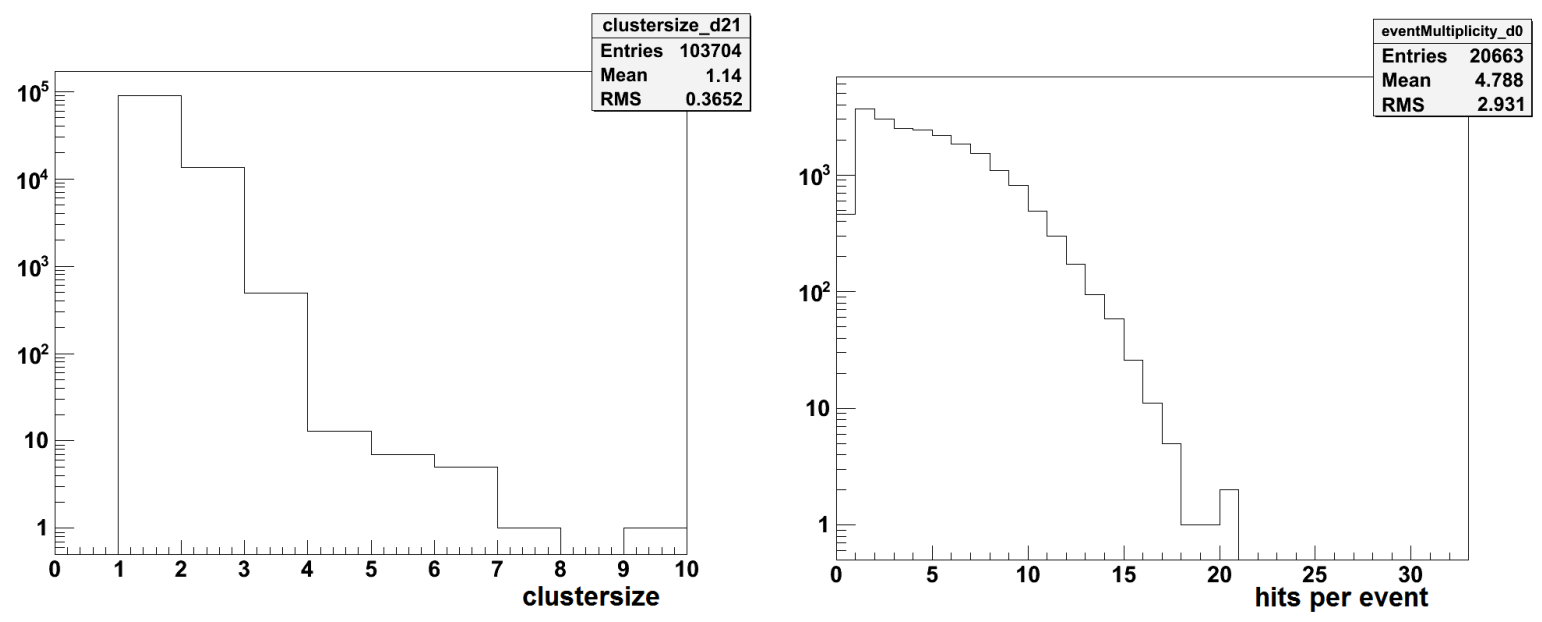

Figure 6.7: Left: Clustersize distribution for a non-rotated APIX sensor ( $\mathrm{x}$ - and $\mathrm{y}$-direction multiplied). The average clustersize is around 1.2, as expected. Right: Event multiplicity of the first telescope plane in the setup. Due to the long integration time, on average around 4.8 clusters are found on this plane per event.

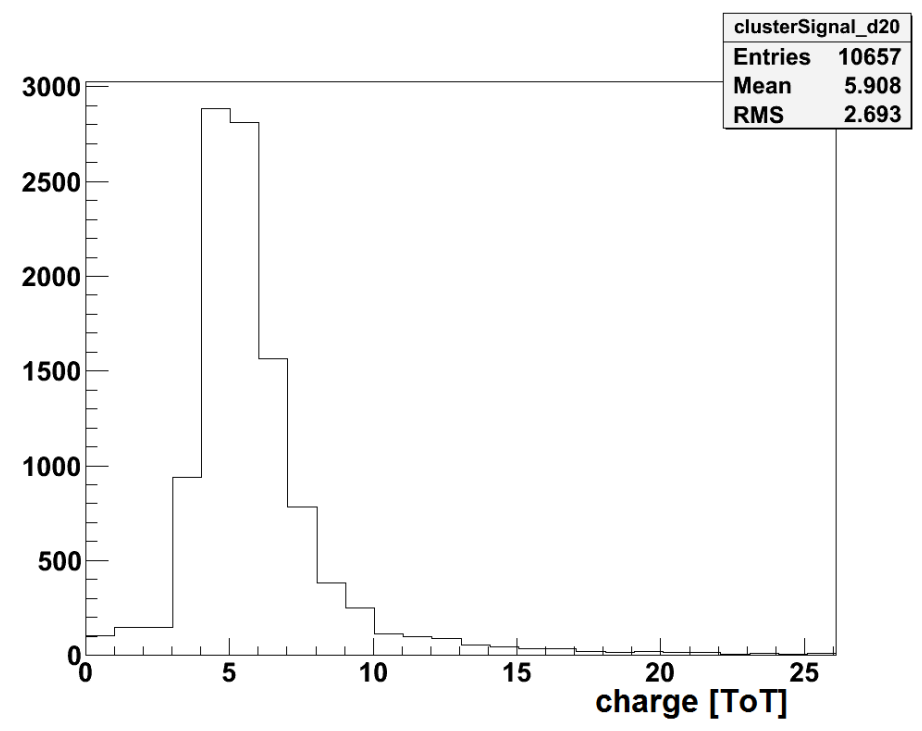

Figure 6.8: Cluster ToT spectrum of an APIX device. The chip has been tuned to a response of 6 ToT units for the expected standard signal of a passing particle. The distribution shows a peak around 6 ToT, with the expected Landau tail.

\section{Hitmaker and Pre-Alignment}

In the third stage of the data reconstruction, the so called "hitmaker" step is executed. This step hosts two main functions, the transformation of hit positions from a local to a global coordinate system and a pre-alignment of all sensor planes in the system.

For transforming to a global coordinate system, the $\mathrm{x}$-axis is set to point along the long pixel direction of the APIX devices, the $y$-axis to the short pixel direction and the z-axis is parallel to the beam. To have a full transformation from a local 2-dimensional coordinate system to a 3-dimensional global system, the z-coordinate has to be read in from the GEAR file, see 6.1.2. Furthermore, the sensor specific rotations are read in and applied, where first the rotation matrices described in figure 6.2 are applied 
and afterwards the three individual rotations around the axes, specified in degrees ${ }^{8}$. This results in a virtual setup, where all sensor planes have the same orientation, which is necessary for alignment and track fitting. Now the previously calculated cluster positions (in local units of columns and rows) are used to determine the cluster position in $\mathrm{x}$ - and $\mathrm{y}$-direction in $\mathrm{mm}$. At this point the center of gravity for each cluster is calculated in global coordinates, where three different options can be chosen. The default option is to take into account the full clusters without specific limitations. Option two is to use a subset of most significant hits (i.e. hits with highest ToT values) per cluster to calculate the center of gravity, where the number of most significant hits per cluster can be set by the user. The third option is to just take hits within a certain frame size, where the size in $\mathrm{x}$ - and $\mathrm{y}$-direction can be given, to calculate the center of gravity position. To keep the framework as flexible as possible the hitmaker processor has separate parts for telescope and APIX settings. The hit collections produced by this processor are the input that is used for the final alignment, as well as for the track fitting.

In the second part of the hitmaker step a pre-alignment is done. The pre-alignment produces correlation plots, where the global hit positions of the single planes are correlated with the first telescope plane ("plane 0"), for the first 10,000 events per run as default value. Here the hot pixels of both, the telescope planes and the APIX devices, are masked. Beside producing correlation plots as control plots for the user, the algorithm calculates hit-by-hit "residuals" for each plane and both directions, comparing event based the global hit positions of telescope plane 0 to the global hit position in the other planes. To not take into account too many outliers, a cut on the width of these hit residuals can be specified for each plane in $\mathrm{x}$ - and $\mathrm{y}$-direction in the steering file (default: $\pm 20 \mu \mathrm{m}$ ). The calculated average shifts of the residuals are then written to a pre-alignment $\mathrm{db}$ file, which is used in the following steps. Since the prealignment works with global coordinates, it can even be used for sensors with different pixel geometries. This method provides rough position corrections on the order of $\sim 100 \mu \mathrm{m}$. Another important function of the correlation plots is the usage as control plots, for checking the provided rotations. In case all values are correct, the resulting correlations should be rising with a slope of 1 . An example for such a correlation plot can be seen in figure 6.9.

\section{Device Alignment with Millipedell}

After calculating rough pre-alignment values in the hitmaker step, these values are loaded in the final alignment processor and applied on the fly to each hit/cluster position to provide a better starting point for the fine alignment. This two staged alignment procedure is necessary, since the device arrangements provided in the GEAR file are values measured by hand. While in z-direction this measurement is possible with an acceptable precision, in $\mathrm{x}$ - and $\mathrm{y}$-direction the plane shifts are very hard to measure. Therefore, first the large scale corrections are determined, before proceeding to the final alignment. Again, hot pixels of the telescope and APIX planes are masked in the alignment.

The final device alignment is aiming towards minimizing the global $\chi^{2}$ of the tracks, which as a consequence also minimizes the residuals. Here a residual is defined as the distance between the measured hit position and the extrapolated position of the fitted track. In a perfectly aligned setup, the width of the residual distribution corresponds to the sensor resolution in the investigated direction and the distribution is centred around 0 . An example of a residual distribution before and after alignment can be seen in figure 6.10 .

As a first step in this processor, straight line fits through all planes are performed in $\mathrm{x}$ - and $\mathrm{y}$-direction independently as input for the alignment tool MillipedeII. To allow a suppression of fake tracks, which would decrease the alignment quality, residual cuts can be set for each sensor plane and each direction

\footnotetext{
${ }^{8}$ This procedure of first rotating in $90^{\circ}$ steps using matrices and additionally applying rotations in degrees will be combined to one set of rotations in a next software version.
} 


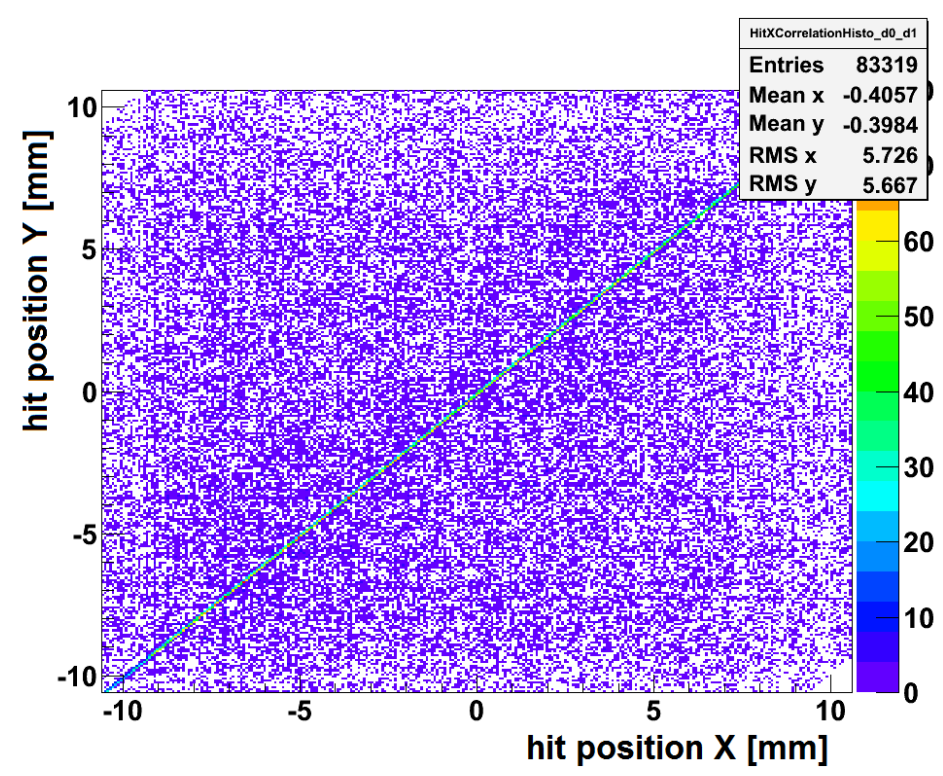

Figure 6.9: Control plot: Hit position correlations in $\mathrm{x}$-direction of the hits on the first and the second telescope plane. The expected rising correlation band is clearly visible (green), as well as the combinatorial background (blue). The shape of the correlations shows that the rotations set in the GEAR file are correct and the correlation band offset is small. Thus, only small corrections will be made by the pre-aligner.

individually. Afterwards, the actual determination of the alignment constants is done using MillipedeII ${ }^{9}$. A key feature of MillipedeII is the simultaneous fit of local and global parameters in order to minimize the $\chi^{2}$ of the tracks [69]. The local parameters are a subset of the measurement matrix in MillipedeII, where in our case a set of local parameters describes an individual track. On the other side, the global parameters describe the whole measurement, which is the final alignment of the whole setup, using all tracks. The idea behind MillipedeII is to treat the problem in a way, such that not the whole global matrix of parameters has to be solved at once, but to fragment the problem to sets of local parameters/tracks (on the order of 100,000 per run). Now the subsets are solved and the results are combined to determine the global parameters.

The tool works in two parts, beginning with the "Mille" part for data collection and preparation. This part provides track candidate residuals and other track-based parameters and writes them to a binary file, which is required by the second part, "Pede". Pede performs a matrix inversion method to solve the alignment problem, until a certain limit is reached, which can be defined by the user. Finally the alignment gives corrections on the $\mathrm{x}$ - and $\mathrm{y}$-position of each plane and the rotations of each device.

As input values for this alignment procedure, the user can set several values beside the previously mentioned residual cuts. Another cut to suppress fake tracks is the usage of DistanceMax, which defines a cone within which the expected track candidate has to be on the subsequent downstream plane, see figure 6.11. Beside that, an upper limit on the number of processed track candidates per event and per total run can be defined, to minimize processing time in case of extreme cluster multiplicities. At the end of the alignment procedure, another $\mathrm{db}$ file is written, containing the fine alignment constants. The pre-align db file and the align db file both are passed on to the final track fitting.

$\mathrm{X}$ residual $[\mu \mathrm{m}]$

\footnotetext{
${ }^{9}$ A detailed description of MillipedeII can be found in http://www.desy.de/ blobel/Mptwo.pdf.
} 

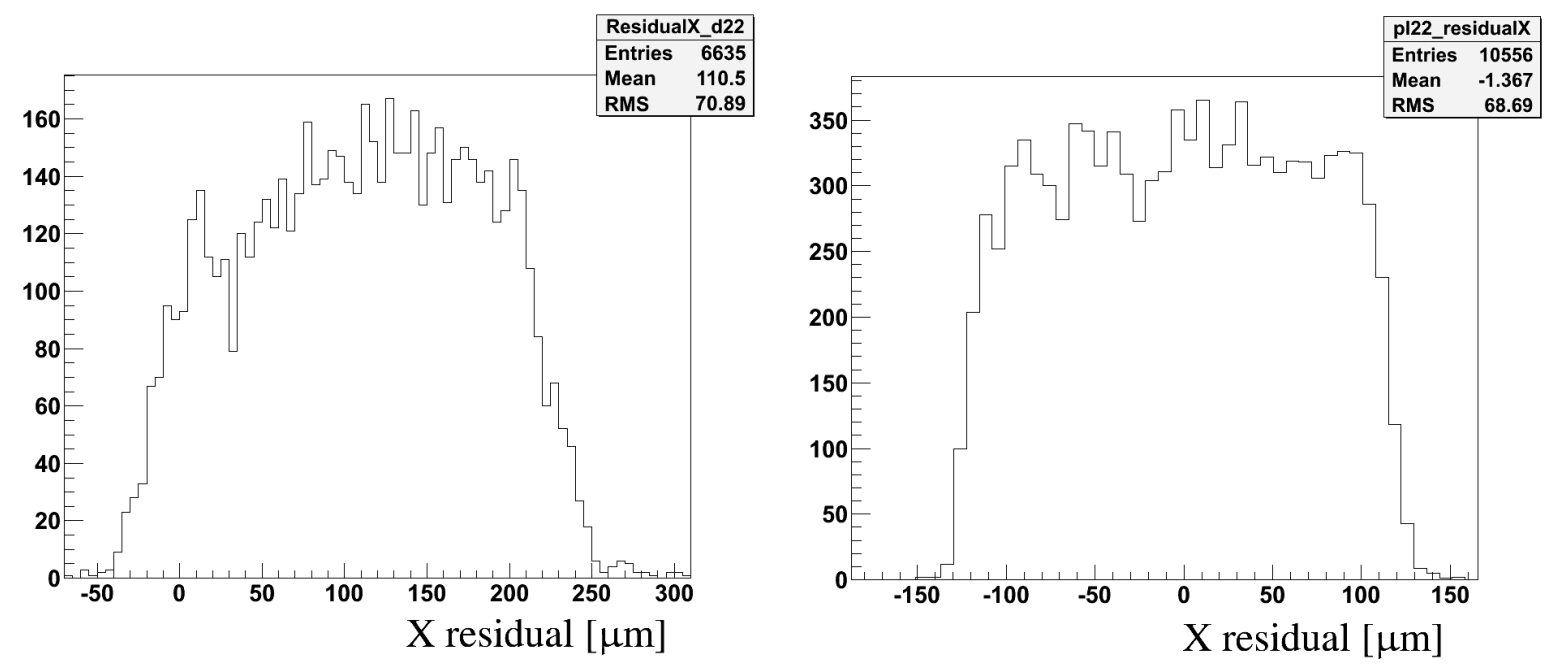

Figure 6.10: Left: residual distribution of an APIX device before alignment. The distribution is shifted from 0 and includes some outliers. Right: residual distribution of the same device after alignment. Now the mean of the distribution is close to 0 and the width represents the spatial resolution of the APIX device in x-direction.
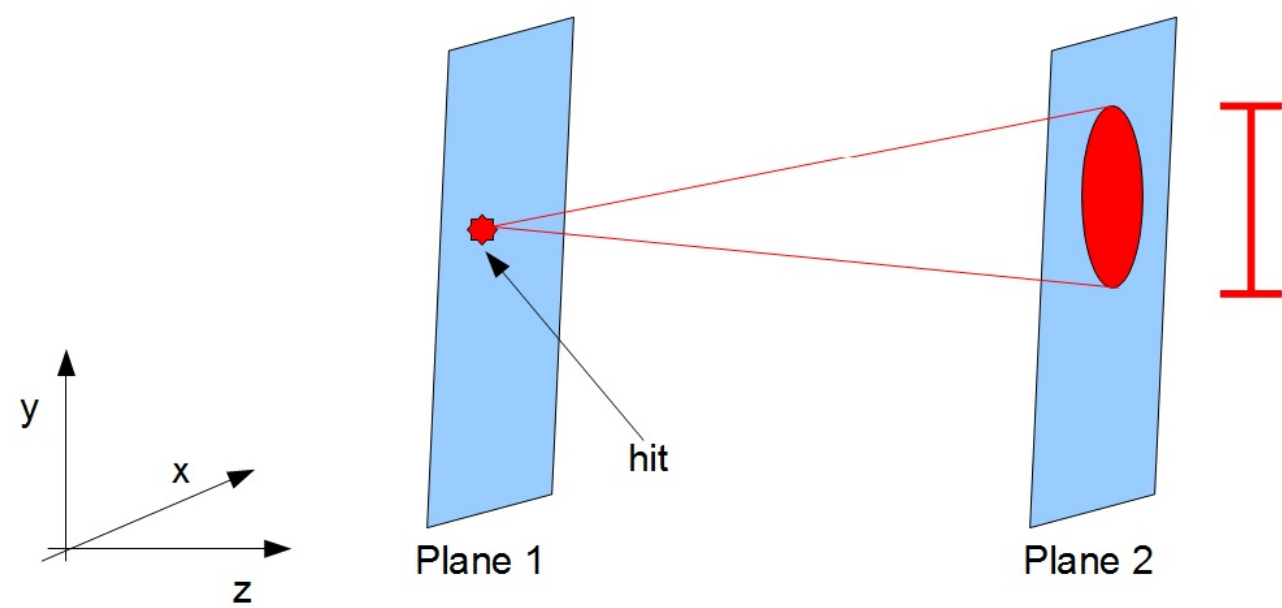

Size of track searching cone

Figure 6.11: One of the background suppression parameters in the alignment step is DistanceMax. This value defines the opening of a cone, within which tracks, extrapolated to the subsequent plane, are being searched for. This value is specified in $\mu \mathrm{m}$ per $10 \mathrm{~cm}$ z-distance between two planes.

\section{Track Fitter}

Track fitting is done in the final reconstruction step. Here, all results of the previous steps are used. First, the hitmaker output is corrected on the fly by the pre-alignment values and afterwards by the final alignment values. After these corrections, the track fitter is executed. As default parameters for the fitting process, a minimum number of four telescope planes and at least one device under test are required to have a hit in the investigated events, to avoid having either low precision tracks or tracks without information for the investigated device. Furthermore, the devices under test are treated as passive layers, to have an unbiased track fit.

The track fitter is based on the concept of a Deterministic Annealing Filter (DAF) [70]. To avoid matching a wrong hit to a predicted track position, the most common way of selecting hits is to calculate their 
residuals. One of the problems, especially for the calculation of the residuals of the first events in a run is, that the track extrapolation algorithm typically suffers from insufficient information, to extrapolate tracks precisely. The DAF approximation uses an iterative procedure to overcome this problem. For each track candidate, the extrapolated position on one plane is calculated, taking into account the information of all other active layers. Then, an assignment probability for every hit to the extrapolated track position is calculated, where all hits falling under a certain assignment probability threshold are ignored in the next iteration ${ }^{10}$. The assignment probabilities for all remaining hits of the iteration are normalized to 1 . Here, each hit observation is the sum of the state vector $\vec{x}_{k}$, multiplied with a mapping matrix $\mathbf{H}_{k}$, and a Gaussian distributed error $\epsilon_{k}$, so that $\vec{m}_{k}=\mathbf{H}_{k} \vec{x}_{k}+\epsilon_{k}$. Then the state vector $\vec{x}_{k \mid k}$ of a layer $k$ is updated, using the prediction $\vec{x}_{k \mid k-1}$, the hit observations $\vec{m}_{k}^{i}$ (with $i=1, \ldots, n_{k}$ ) and the assignment probability $p_{k}^{i}$ :

$$
\vec{x}_{k \mid k}=\vec{x}_{k \mid k-1}+\mathbf{K}_{k} \sum_{i=1}^{n_{k}} p_{k}^{i}\left(\vec{m}_{k}^{i}-\mathbf{H}_{k} \vec{x}_{k \mid k-1}\right)
$$

This process is repeated until the assignment probabilities converge to their final values and the final track fit for the investigated event can be performed.

\subsection{Analysis Tools - TBmon}

While the track reconstruction framework hosts a huge set of functionalities, some of them for devices that are not of ATLAS pixel type, the detailed analysis of the devices under test happens in the analysis tool "TBmon". The roots of this tool can be found in a functionality as online monitor for testbeams of the 3D sensor community. In those days TBmon produced some basic histograms on the fly. To have a standalone $\mathrm{C}++$ based framework, which can be run on every standard PC, only having $\mathrm{ROOT}^{11}$ as external dependency, TBmon was substantially extended and modified towards an offline analysis tool. During the time of IBL testbeam campaigns, TBmon was chosen to be the official analysis tool for all IBL, 3D, planar and diamond testbeams and is still in use for ongoing analyses. Its structure allows to analyse all kinds of sensor devices, which are based on a pixelated rectangular shape. The general structure of TBmon is shown in figure 6.12.

The input for TBmon are tbtrack-files in a ROOT file format, which are produced by the fitter processor

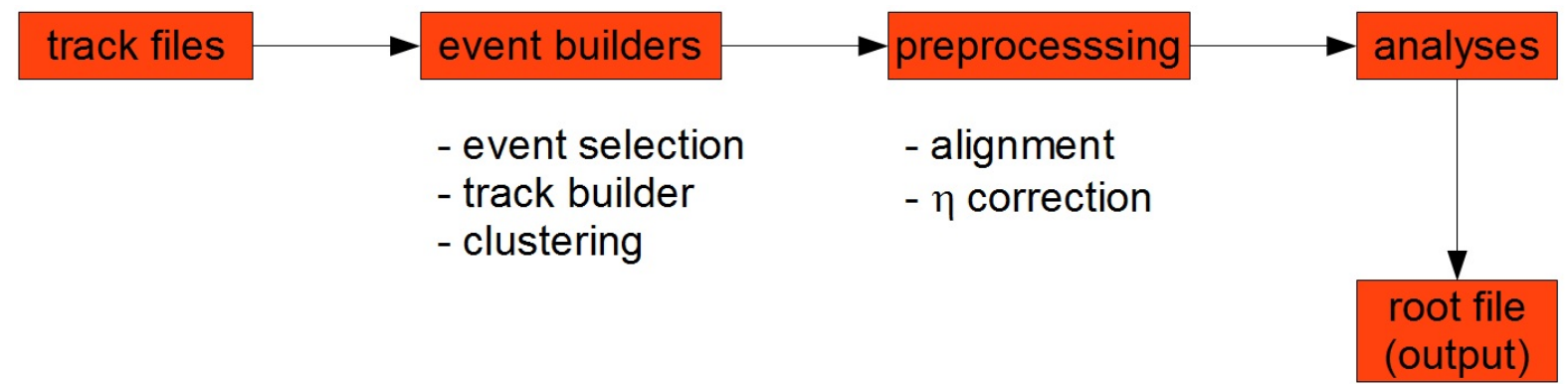

Figure 6.12: General structure of TBmon: track files are read in, a preprocessing is performed on the dataset, followed by the analyses and finally the results are written into a ROOT file.

\footnotetext{
${ }^{10}$ Note: these hits are not masked for the whole fitting process, but only for the subsequent iteration, they might be considered in later iterations again.

${ }^{11}$ root.cern.ch
} 
of EUTelescope. These files contain several trees, where the entries of the trees provide the necessary information about the devices under test (DUT). Examples for such trees are a hit tree, holding eventbased vectors with all the DUT hits, the trigger ID, DUT ID of the hit device(s), the ToT encoded charge value of each hit and the timing information in units of LVL1 (see testbeam dictionary A.1) and a track tree, providing information like the fitted track positions and a timing tree. Altogether the tbtrack files contain all relevant information to perform the DUT analyses without the need for any other input, except a description of the DUT type (e.g. FE-I4 style module).

TBmon offers the possibility to combine several runs for one analysis, which were taken under the same conditions. Here same conditions especially means that the DUTs were the same, neither the tuning of the devices nor the applied bias voltage changed and the temperature was stable within the reachable limits. Before the analysis of the set of runs (or an individual one) begins, different calibrations and cuts can be applied. This procedure is schematically shown in figure 6.13, while the most important processes are described in the following.

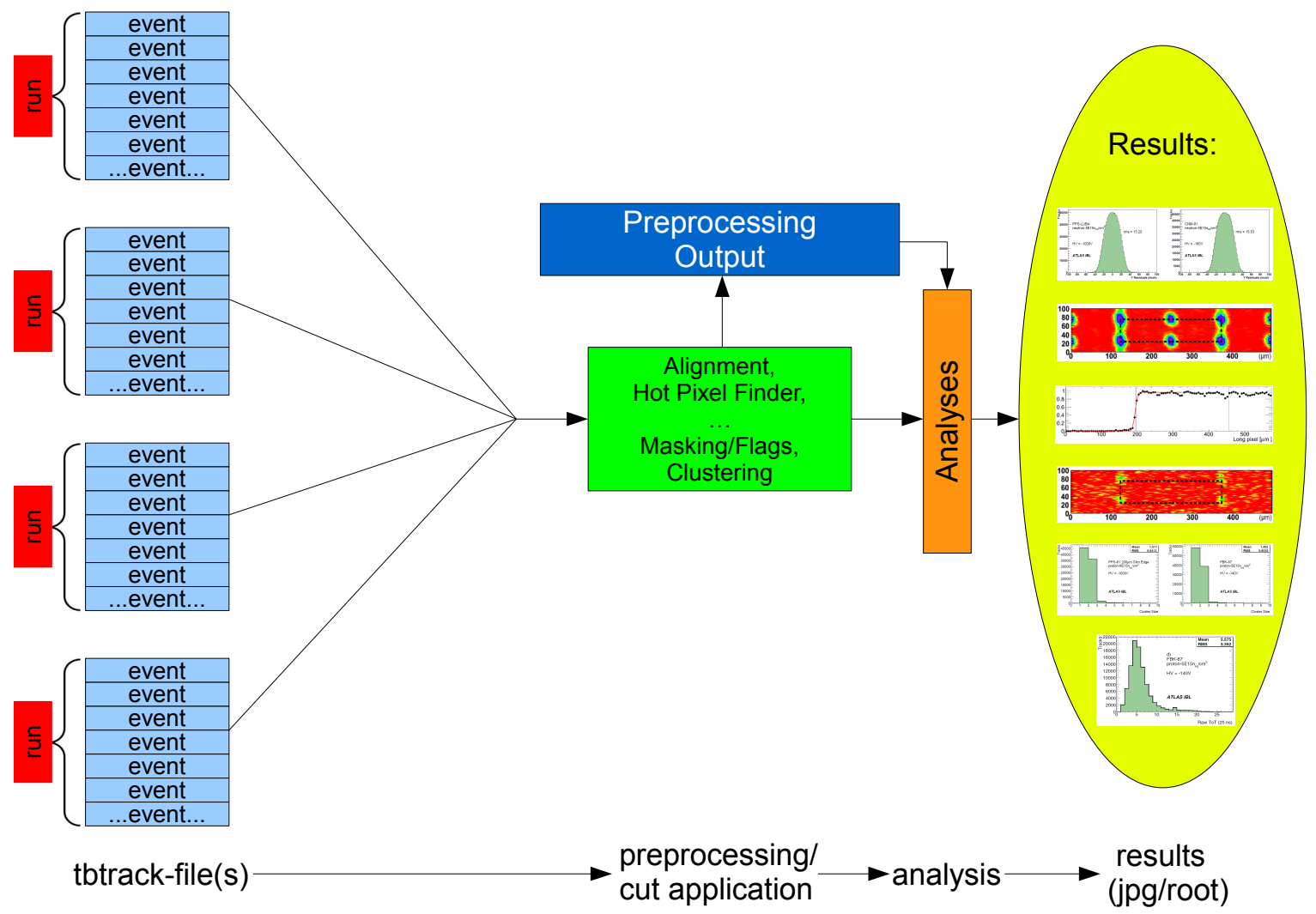

Figure 6.13: Schematic structure of TBmon. Several tbtrack-files, hosting a certain number of events, can be combined for processing, if they were taken under the same conditions. Different preprocessing sequences are performed on the data and cuts can be applied, followed by the analysis processors. The results can be written into single graphics files (e.g. jpg format) or collected in a ROOT file. 


\subsubsection{Eventbuilders and Preprocessing}

In a first step, the raw data is read in by TBmon to run the eventbuilders, which perform the track building, clustering and create event structure used in TBmon. The naming scheme is ambiguous at this point between the reconstruction framework and the offline analysis. The TBmon eventbuilders read in data event by event from the tbtrack file, where events are separated by their trigger IDs (see section 5.1.3). Each event contains the raw hits of the devices under test in column/row coordinates and in $\mu \mathrm{m}$ in the global coordinate system, the track positions extrapolated to the DUTs, cluster data and TLU timing information. Out of this dataset, TBmon reads in the raw hits in local coordinates, the extrapolated track positions and the timing information to perform the eventbuilder functions listed before. Once the eventbuilder tasks are completed, a set of preprocessing steps can be executed, namely hot pixel finding, alignment and $\eta$ correction. The detailed functionality of the eventbuilders and preprocessors is listed in the following subsections.

\section{EUBuildTrack}

The first eventbuilder executed by TBmon is EUBuildTrack, where the tbtrack file is opened to perform track building. As criterion for a matched track on a device, first all other devices under test are searched for matches. Only in case a match in at least one of the other planes was possible, the investigated device is checked for hits that can be matched to tracks. This procedure is realized in a loop called "looper", which is executed device by device. In figure 6.14, the idea behind the TBmon looper is shown schematically, since the concept is important for doing in-time analyses. As described in section 5.1.2, the DUT buffers' data storage space is only sufficient for a very short time, compared to the integration time of the telescope sensors. Thus, the described procedure is the only way to check for in-time tracks, using the current setup.

For each of the track match candidates found in this procedure, a set of cuts can be applied. The available cuts and their default values are:

- $\chi^{2}$ cut with a default value of 999

- matching radius, default: $1.5 \mathrm{x}$ pixel pitch

- number of other planes required to have a matched hit, default: 1

- LVL1 timing cut, default: 0 - 10 in units of $25 \mathrm{~ns}$

The listed "LVL1 timing" stands for the latency between the trigger signal and the read out of the data. This latency value can be tuned, thus a peak in the LVL1 distribution is expected, as shown in figure 6.15 (left). Hits registered outside of the peak region can be counted as noise hits, which appear equally distributed in time. For comparison the LVL1 distribution of a very noisy sample is shown in figure 6.15 (right). Thus, the cut has to be adapted for each dataset individually.

As soon as the looper finds a track that fulfills all the requested characteristics, it jumps to the next event - which is one of the main differences, compared to the developments described in 6.2.3. As last important feature, EUBuildTrack sets individual flags for each processed track. Properties like whether a track was matched at all and the type of the extrapolated region a track hits on a DUT (hitting the edge region, a region of masked pixels or the central region of a device) are categorized by these flags.

Following this procedure, each investigated device is searched for hits that can be matched with the given specifications to a track. Important is, that only the tracks are checked for the possibility of being 
matched, the corresponding hits are not labelled yet. The classification of the hits is done in the next step, the TBmon internal clustering.

After the track building is done, a cluster search is performed on all devices. The motivation for redoing

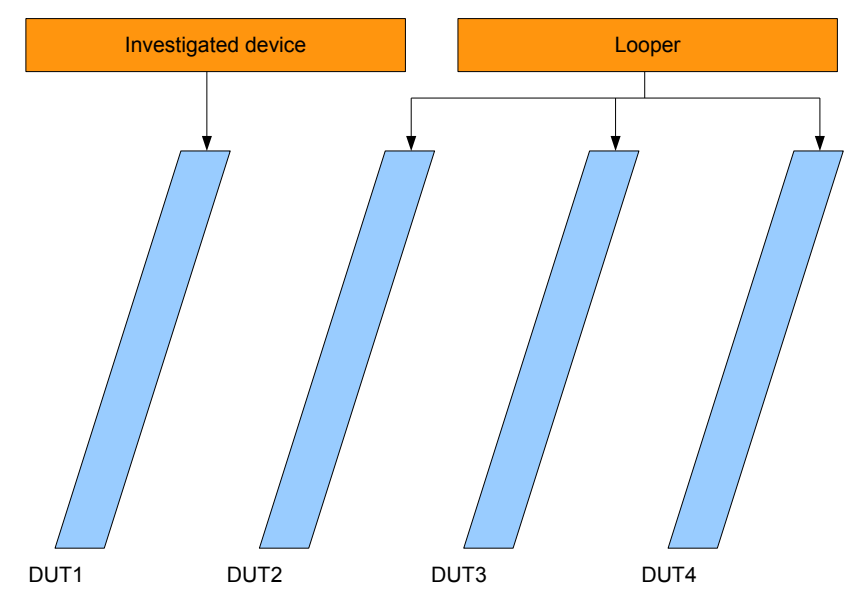

Figure 6.14: Schematic drawing of the TBmon looper functionality, which is the central part of the track builder. The looper reads information for all DUTs, except the investigated one, to check for in-time hits in the investigated DUT and match them to the respective track.

the clustering in TBmon, instead of loading the clustering information gained during track reconstruction, was to have the possibility to use different cluster algorithms. This feature can be useful in case the device geometry requires a special treatment for specific regions of the sensor. In the clustering step, the first hit in a plane is taken, all eight neighbour pixels are searched for entries and in case a hit is found, the search is continued until no further neighbouring hits are left and the hits are assigned to one cluster. In case a cluster is found, it is stored, such that the clusters can easily be accessed by the analysis processors. To distinguish different cluster qualities, in a second step the clusters are either labelled with additional flags or discarded, depending on their properties. Particularly flags are used to indicate whether a cluster can be matched to a track in the corresponding event or to mark that a cluster contains a pixel, which is masked by the hot pixel killer. By default clusters containing a masked pixel are disregarded in further analyses, which might introduce a bias, since problematic pixels are ignored from this point on.

\section{HotPixelFinder}

In a standard analysis, the first processor that is manually executed by the user is the HotPixelFinder. This processor analyzes the LVL1 timing distributions of the processed data and creates an out-of-time hitmap per DUT. The available cut for the hot pixel finder is:

- occupancy cut, default value: $5 \cdot 10^{-4}$

Two different functions scan all pixels for either showing no hit at all in the whole dataset, these are marked as dead pixels, or for pixels exceeding the occupancy cut, which are counted as noisy pixels. In case a device has several noisy pixels, they will fire at random times, which results in a noise floor, when histogramming the distribution of recorded LVL1 values. A comparison of the LVL1 distribution of a 
device performing as expected and a very noisy device is shown in figure 6.15. Pixels falling in one of the two categories (dead/noisy) are written into a text file, which is read in at the beginning of the next EUBuildTrack cycle. To apply the pixel masks, EUBuildTrack is executed again, before the following analysis steps can be performed.
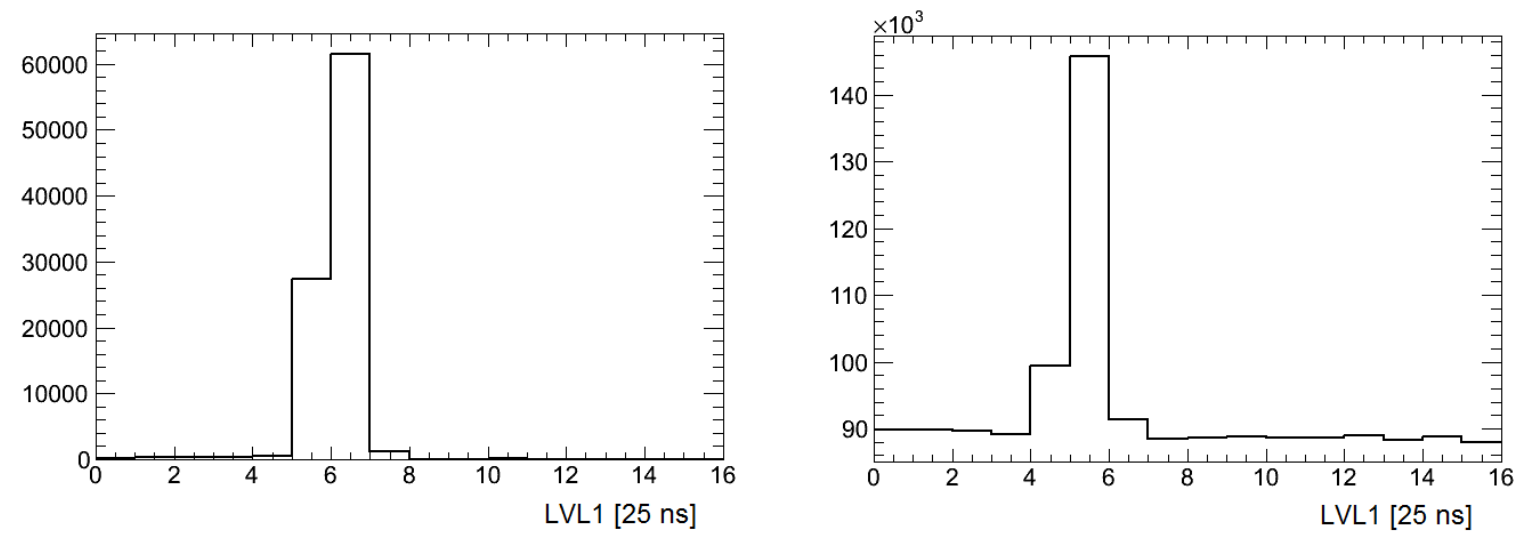

Figure 6.15: Left: expected LVL1 distribution of a tuned ATLAS pixel device. The hits are registered after a certain latency tuned to 6 clock cycles ( 25 ns per LVL1), plus and minus one LVL1 bin, due to effects like timewalk. Right: LVL1 distribution of a device dominated by noise hits. A uniformly distributed noise floor is visible, having a peak around the tuned latency value (note: here the y-axis starts at 85000 entries).

\section{CheckAlign}

TBmon includes its own DUT alignment processor called CheckAlign. To achieve an increased alignment precision, using the tracks fitted through the telescope and the extrapolated positions on the DUTs coming from the track reconstruction framework, this step can be performed in TBmon optionally. Since the alignment is sensitive to the quality of the used tracks, several cuts and masks are applied in the CheckAlign processor. The applied cuts are:

- $\chi^{2}$ cut with a default value of 999

- matching radius, default: $1.5 \times$ pixel pitch

- number of other planes required to have a matched hit, default: 1

- LVL1 timing cut, default: 0 - 10 in units of $25 \mathrm{~ns}$

- restriction to the central region of the sensor

- track is not extrapolated to a masked pixel/cluster

- event contains at most one cluster per plane

- if already available: apply eta correction

An eta correction (see next step) is applied to the clusters, to achieve the highest possible alignment quality.

For all events passing the cuts, the track residuals are calculated and filled in a histogram. The track 
residual is the distance between the extrapolated/expected track position on the device and the charge weighted, eta corrected - if already available - cluster center position: Res $=$ TrackPos - ClusterPos.

After the whole dataset is processed, a set of histograms is available, showing the residual against hit position for all possible combinations ( $\mathrm{X}$ residual vs $\mathrm{X}$ position, $\mathrm{X}$ residual vs $\mathrm{Y}$ position etc), see figure 6.16. These histograms can be used to get an impression of the alignment quality. For a perfect alignment, the histograms should show a horizontal line at a value of 0 . Thus, the shown histograms indicate the required shift per device to have it aligned as well as possible and show whether the device has to be rotated in the geometry description. In case the line has a slope, angle corrections have to be applied to the reconstruction and a new tbtrack file has to be produced.

To calculate the shifts of the DUTs in $\mathrm{x}$ - and $\mathrm{y}$-direction, the checkalign processor determines the mean of the residual distributions in $\mathrm{x}$ - and $\mathrm{y}$-direction and writes these values into a text file, which is read in by the Translator, when running EUBuildTrack again. After the alignment in TBmon is completed
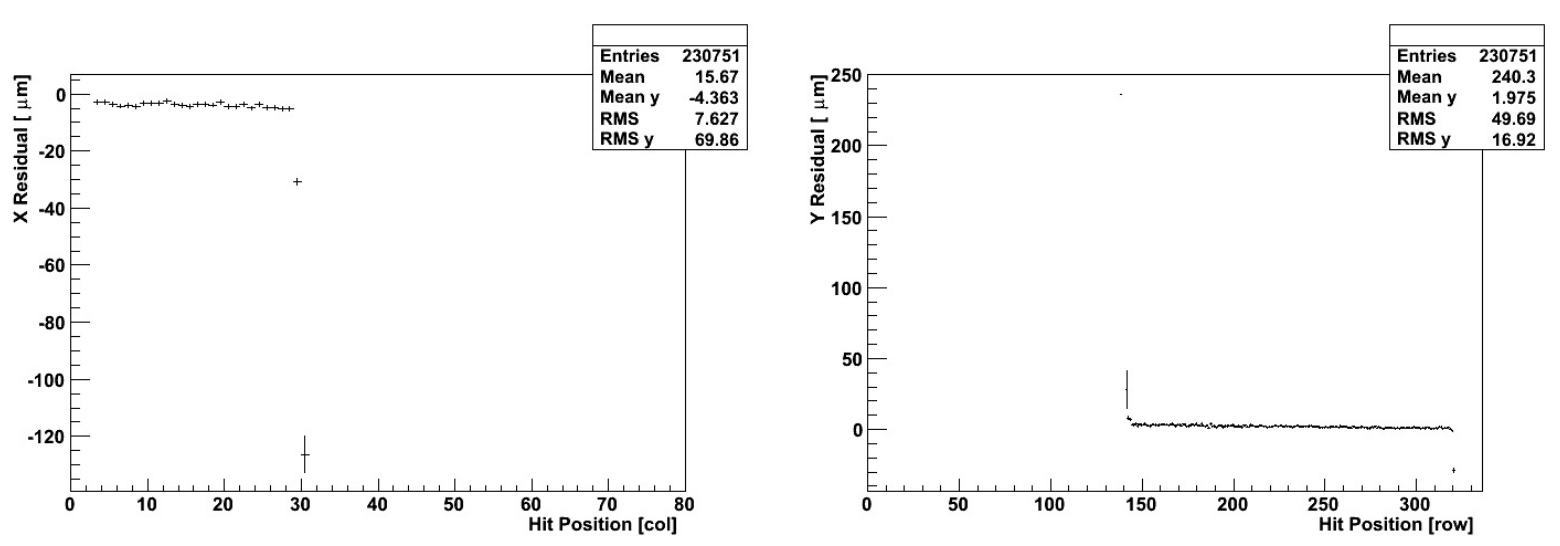

Figure 6.16: Exemplary histogram output of the TBmon internal CheckAlign processor. After applying several quality cuts, the averaged residual is plotted against the hit position for the four possible combinations: residual $\mathrm{X}$ vs hit $\mathrm{X}$, residual $\mathrm{Y}$ vs hit $\mathrm{X}$, residual $\mathrm{X}$ vs hit $\mathrm{Y}$ and residual $\mathrm{Y}$ vs hit $\mathrm{Y}$. The data points far off the horizontal lines are at the edge of the scintillator region, thus they have low statistics and large errors.

and the corrections are applied, the residuals can be histogrammed separately for $\mathrm{x}$ - and $\mathrm{y}$-direction, as shown in figure 6.17. As visual check for the alignment quality, a box fit with and without slope in the plateau region is applied to the distributions. Ideally, the box fit without slope should perfectly describe the residual distribution. The fit with a slope in the plateau region is used to check for any unexpected trends in the distribution. For a good alignment, the distribution is expected to be centred around 0 and to have a width corresponding to the pixel pitch. The RMS of the found distribution can be compared to the expected resolution of the investigated device ${ }^{12}$, given by Resolution $=\frac{\text { pixel pitch }}{\sqrt{12}}$. The resulting values for a FE-I4 based device are $72.2 \mu \mathrm{m}$ in the long direction and $14.4 \mu \mathrm{m}$ in the short direction. As an exemplary check, comparing the calculated RMS values, which are printed in the histograms, to the expected value for the long pixel direction, the resolution of the device fits the expected value. For the short pixel direction the small deviation from the expected value can be explained by a not yet perfect alignment in y-direction of the exemplary device.

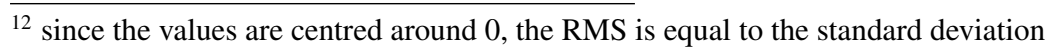



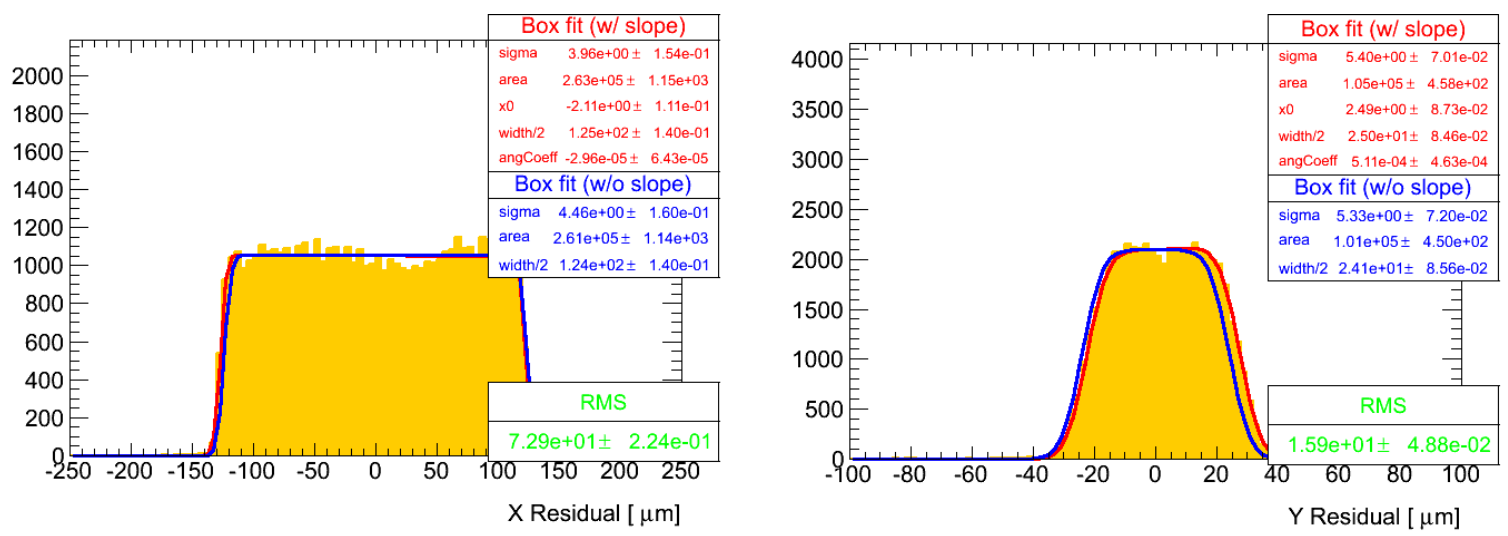

Figure 6.17: Exemplary residual distributions in $\mathrm{x}$ - and y-direction for a FE-I4 chip based device. A box fit with and without slope in the plateau region is used to determine the width of the distribution, the mean value and the RMS. Note: the structure visible in the plateau regions of the shown examples can be explained due to the different residual distributions for different cluster sizes and the coarse charge resolution of the FE-I4 chip, which has an influence on the calculated charge weighted positions. In addition, the not perfect alignment of the devices in this exemplary dataset influences the flatness of the distribution.

\section{Translator}

A preparatory function that is automatically executed each time EUBuildTrack is called, is the Translator. Its only function is to load the alignment corrections to the track builder step, to provide the calculated alignment shifts to the tracks. These corrections can either originate from values the user enters manually in a configuration file or from the text files created by CheckAlign. By default only the CheckAlign corrections are used.

\section{GetEtaCorrection}

The third and last processor in the set of preprocessing options is GetEtaCorrection, in which the eta correction is calculated for each device individually [71]. An eta correction compensates for the fact that the charge division does not happen in a linear way between the hits of a cluster. The reason behind the non-linearity is the lateral diffusion of the charge created by incident particles. In case a particle crosses an idealized sensor at perpendicular incidence exactly in the middle between two pixels, the charge will divide in equal fractions into both pixels. Since the value eta is defined as $\eta=\frac{Q_{R}}{Q_{R}+Q_{L}}$ with $Q_{R / L}$ being the charge detected in the right/left pixel, the $\eta$ value will be 0.5 in the central region between the pixels. For particles traversing close to the central position the value changes slowly with increasing distance from the central position, until from a certain point on all charge is detected either in the left or right pixel. This results in having two peaks in the eta distribution, at values of 0 and 1 . In reality an additional noise contribution smears the distribution out a bit, which especially broadens and shifts the peaks as shown in [71]. Using the resulting eta value distribution, a correction function can be determined for each possible value of $\eta$ by integrating the distribution up to each determined value and normalizing it. To achieve an eta correction function within TBmon, the GetEtaCorrection processor uses events with exactly one cluster per device and event, where the cluster has to be located in the central region of the device and pass all other criteria that are required by the previously described CheckAlign processor. On top of these criteria, only clusters are used with a maximum clustersize of two, since $\eta$ is only defined for 2-hit clusters. The achieved eta correction can be applied when running the event builders again and improves the alignment quality of CheckAlign due to the more precise cluster positions. The final 
cluster position is: $x=x_{\text {left }}+$ Pitch $\cdot f(\eta)$.

Thus, the typical running order of TBmon is:

- HotPixelFinder

- CheckAlign with applied pixel masks from previous step

- GetEtaCorrection with applied masks and alignment corrections

- CheckAlign with applied masks and eta corrections

After this procedure is finished, the central analysis part of TBmon can be executed.

\subsubsection{Analysis Processors}

In the following, an overview of the most important analysis processors provided within the TBmon framework, as they were used for the IBL, 3D silicon, planar silicon and diamond testbeam analyses, is given. Generally, the analyses are performed after all preprocessors were executed and the resulting corrections are available. The analysis processors do not interact with each other, but only use the output of the previously described eventbuilders and preprocessors. Thus, the analyses can be performed in arbitrary order and individual analysis processors can be skipped or added to the execution list.

The following list of analysis processors is given in alphabetical order. The shown plots are meant as examples, to illustrate the relevant output of each processor, thus no conclusions on the performance of the devices are drawn from the histograms in this section. Different datasets were used to produce the exemplary histograms, since some effects are only visible for a certain device type or under certain operating conditions.

\section{BeamProfile}

The BeamProfile processor provides some basic information on the beam and devices characteristics. For the used dataset, a filter on some basic flags set by the eventbuilders, can be added, while per default just the raw tracks are used. In the following, for each processor an overview table is provided, listing on the left the cuts used by the processor and on the right the histograms produced after the cuts were applied. Within the shown tables, the list of cuts is meant inclusive when going to the entries in the subsequent row.

\begin{tabular}{|l|l|}
\hline cut & histogram \\
\hline \hline- & $\chi^{2}$ distribution \\
\hline$\chi^{2}$ cut on tracks & $\chi^{2}$ distribution \\
\hline & trackmap \\
\hline
\end{tabular}

Table 6.1: BeamProfile: overview of the available cuts and produced histograms.

As output, the processor displays characteristics of the raw tracks, like the $\chi^{2}$ distribution without any applied cut, as shown in figure 6.18. The same distribution can be histogrammed after the cut was applied. Knowledge of the $\chi^{2}$ distribution with and without applied cut can be useful to properly adjust the cut value without running the processor twice, in order not to loose too many tracks due to a not 


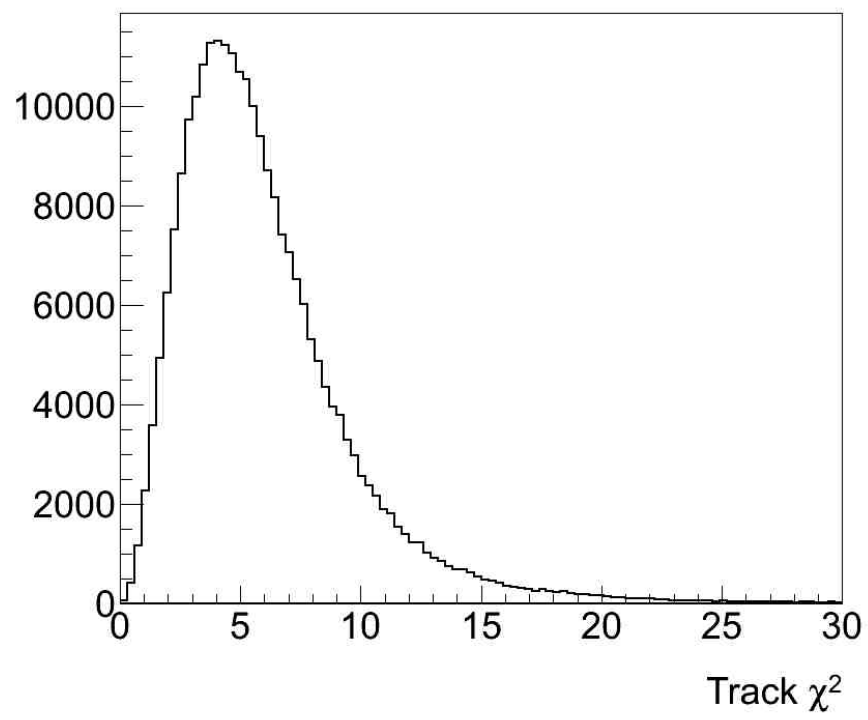

Figure 6.18: Typical $\chi^{2}$ distribution of the tracks, used for the analysis of a FE-I4 based device. This distribution shows the whole $\chi^{2}$ spectrum, to give the user a cross-check for the applied cut.

perfect cut. A further example of data quality information that can be displayed by this processor is shown in figure 6.19. Here, a track map is created, showing all tracks being in the central region of the sensor and passing the $\chi^{2}$ cut. The track position in $\mu \mathrm{m}$ is filled in the histogram, to get an impression of the beam profile, especially the size of the beam spot.

\section{ClusterChecker}

The ClusterChecker processor provides a set of histograms, summarizing the results of the TBmon internal clustering. For some of the produced histograms, only clusters are taken into account that can be matched to a track, to have an overview of the matched clusters properties. Here, the requirement on the used track is that it can also be matched in a reference plane and that it passes the $\chi^{2}$ cut. The cutflow of the processor and the produced histograms are:

\begin{tabular}{|l|l|}
\hline cut & histogram \\
\hline \hline $\begin{array}{l}\text { tracks through central region } \\
\chi^{2} \text { cut on tracks }\end{array}$ & number of hits per event \\
\hline match in reference plane & track map \\
\hline
\end{tabular}

Table 6.2: ClusterChecker: overview of the available cuts and produced histograms.

As shown in figure 6.20 one of the control plots produced by this processor shows the number of hits per event, detected in the investigated device over the whole dataset. Beside that, the cluster size of matched clusters is histogrammed, as well as the size of clusters that were not matched to a track, see 6.21. This comparison of matched versus un-matched cluster sizes is biased due to the fact that EUBuildTrack can only handle one track per event. Thus, some clusters can be caused by an incident particle and have a 


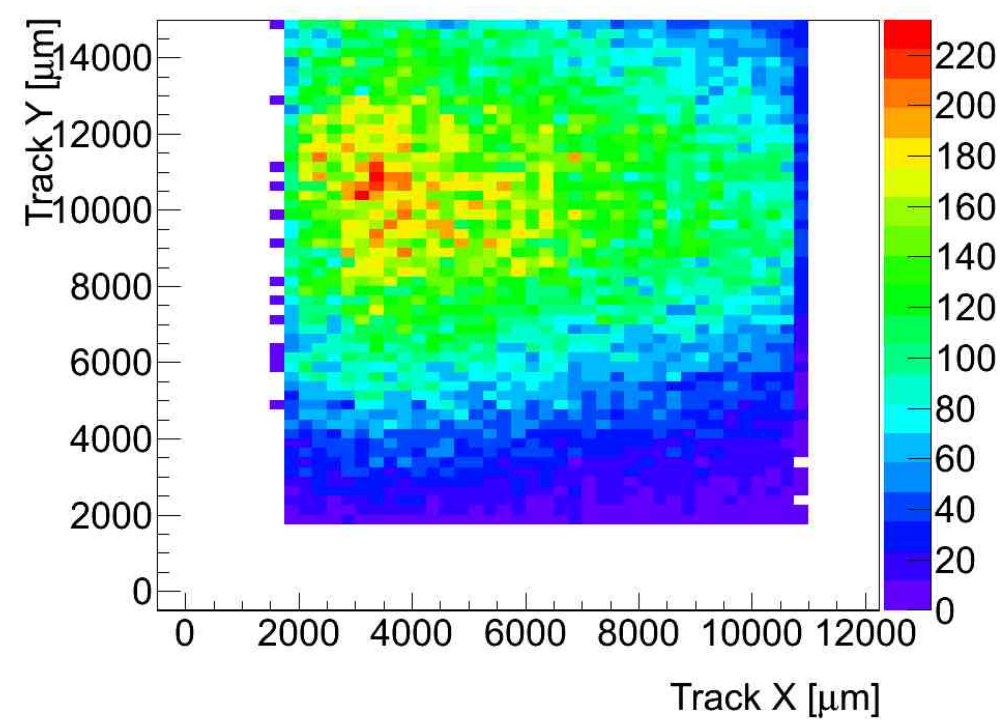

Figure 6.19: Track map created by the BeamProfile processor, showing all tracks going through the central region of the device that pass the $\chi^{2}$ cut. This track map gives an impression of the beam spot size and an indirect impression of the scattering magnitude. Here the track map shows that the particle beam was focussed a bit towards the upper left of the trigger scintillator area.

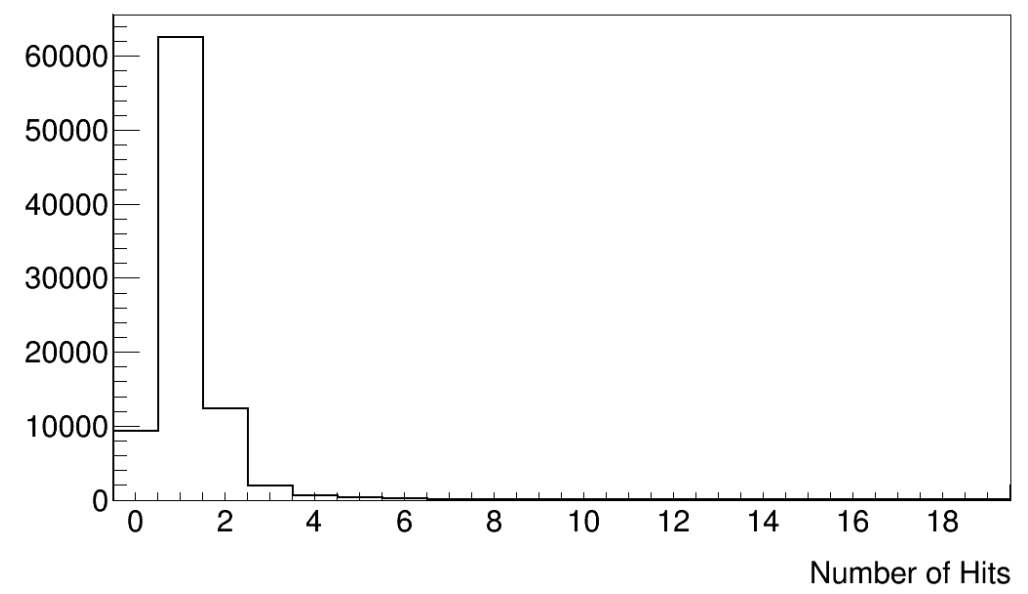

Figure 6.20: The histogram shows the number of hits per event. It can be seen, that events can occur, having no hit at all because of a track that missed the device, but the expected peak for a hit multiplicity.

corresponding track, but are just not matched by TBmon. This feature is repaired in the new version TBmon II, described in section 6.2.3.

An impression of the number of clusters per event on the investigated device is given in figure 6.22. The number of entries for a multiplicity of 0 corresponds to the number of events in which a track was matched in other devices, but either missed the investigated device or was not detected. For multiplicities of 2 and more, TBmon can only treat one cluster of the event, as explained before. The cluster multiplicity histogram again gives an impression of the data quality, since high multiplicities (higher than $\approx 4-5$ rule of thumb) lead to ambiguities in the alignment and track fitting and therefore decrease 

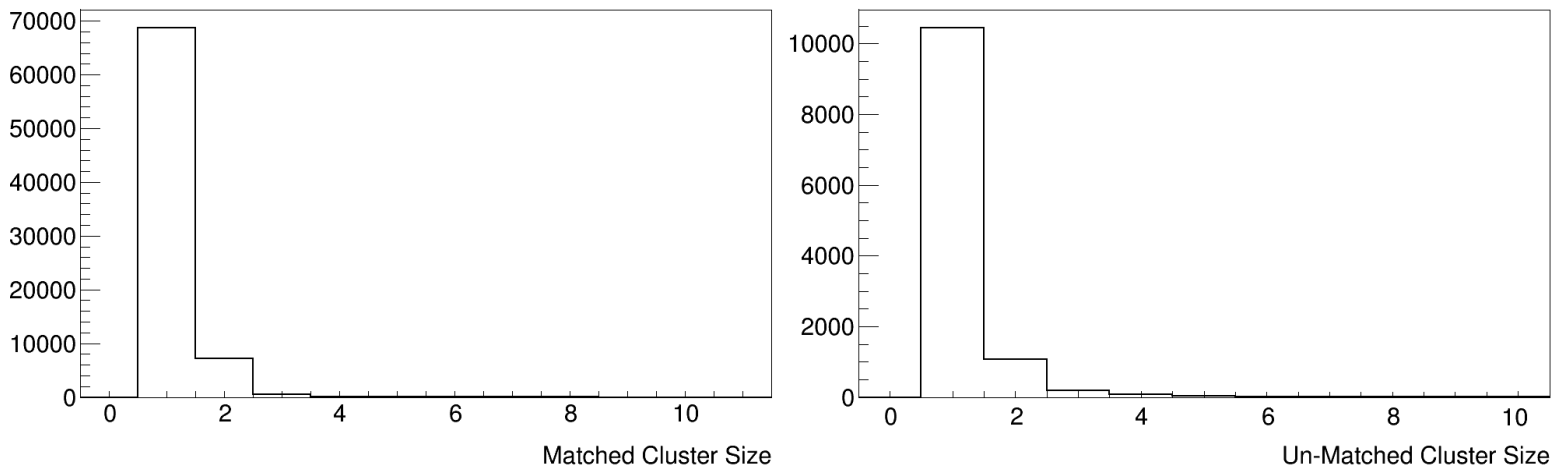

Figure 6.21: Left: cluster sizes detected in the investigated device, taking into account only clusters that can be matched to a track, where the same track has to be matched also in a reference device. Right: cluster size distribution of clusters without a matched track. The relatively high number of un-matched clusters can be explained by the fact that TBmon only treats one track per event.

the quality of the dataset.

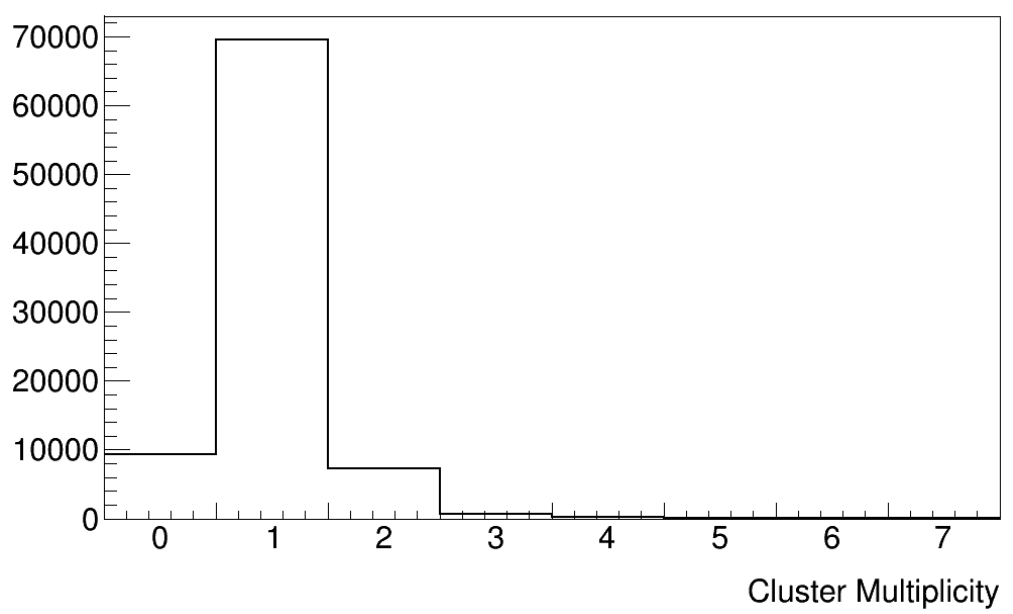

Figure 6.22: Exemplary histogram showing the cluster multiplicity of a device. As for the hit multiplicity, a value of 0 is caused by events in which a track was matched to other devices in the setup, but either missed the investigated device or was not detected.

\section{Efficiency}

One of the most interesting properties of a device in testbeam measurements is the tracking efficiency. To determine the efficiency out of testbeam measurements, an unbiased track selection has to be performed, which is partly caused by the definition of the efficiency itself and partly by the setup. 


\begin{tabular}{|l|l|}
\hline cut & histogram \\
\hline \hline $\begin{array}{l}\text { tracks through central region } \\
\chi^{2} \text { cut on tracks }\end{array}$ & raw hitmap \\
\hline match in reference plane & in-time hitmap \\
\hline track does not hit masked pixel & efficiency map, in-pixel efficiency \\
\hline
\end{tabular}

Table 6.3: Efficiency: overview of the available cuts and produced histograms.

As shown in the table, for the efficiency calculation only tracks are taken into account, which go through the central region of the sensor and pass the $\chi^{2}$ cut. Furthermore, a match in the reference plane is required, which is unavoidable due to the different active time spans after a trigger signal of the DUTs and the telescope planes ${ }^{13}$. To illustrate the filtering effect of the matched hit criterion, in figure $6.23 \mathrm{a}$ raw hitmap can be compared to a map showing only matched in-time hits. Then the number of these in-
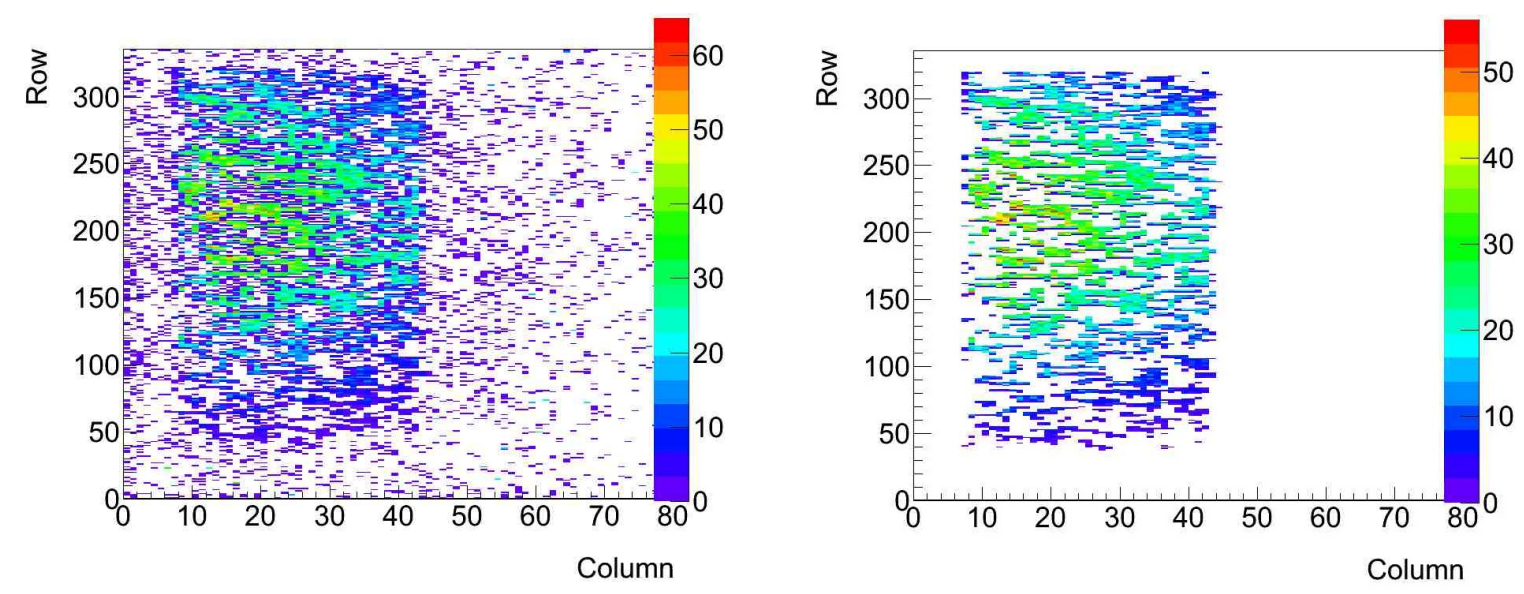

Figure 6.23: Left: Hitmap of a device without applying any cuts, just counting the number of hits per pixel cell. Right: Hitmap of the same device after applying timing cuts and only taking into account hits that can be matched to a track. Note that all hits outside of the projected trigger scintillator area vanish.

time tracks ${ }^{14}$, which have a matched cluster in the investigated device within the given matching radius, is counted. This is the total number of real tracks detected by the investigated device. To actually calculate the efficiency of the investigated device, the number of tracks in the whole processed dataset is counted, which have a matched cluster in a reference plane. This number is the amount of tracks that also the investigated device should have detected. Using this information, the efficiency and the binomial error on the efficiency are calculated through:

$$
\begin{gathered}
E f f=\frac{\text { number of tracks with matched hits }}{\text { total number of in-time tracks }} \\
\sigma(\text { error })=\sqrt{\frac{E f f \times(1-E f f)}{\text { number of tracks }}}
\end{gathered}
$$

\footnotetext{
${ }^{13}$ usage of a FE-I4 chip based device as trigger plane to have a timing resolution of the trigger is work in progress

${ }^{14}$ Here in-time tracks are those tracks, which arrived within the active buffer time of the reference plane, as described in section 6.2.1.
} 
The systematic error is not considered here. The positions of the matched tracks used for this calculation, are histogrammed in figure 6.24. This histogram is useful, together with the device efficiency

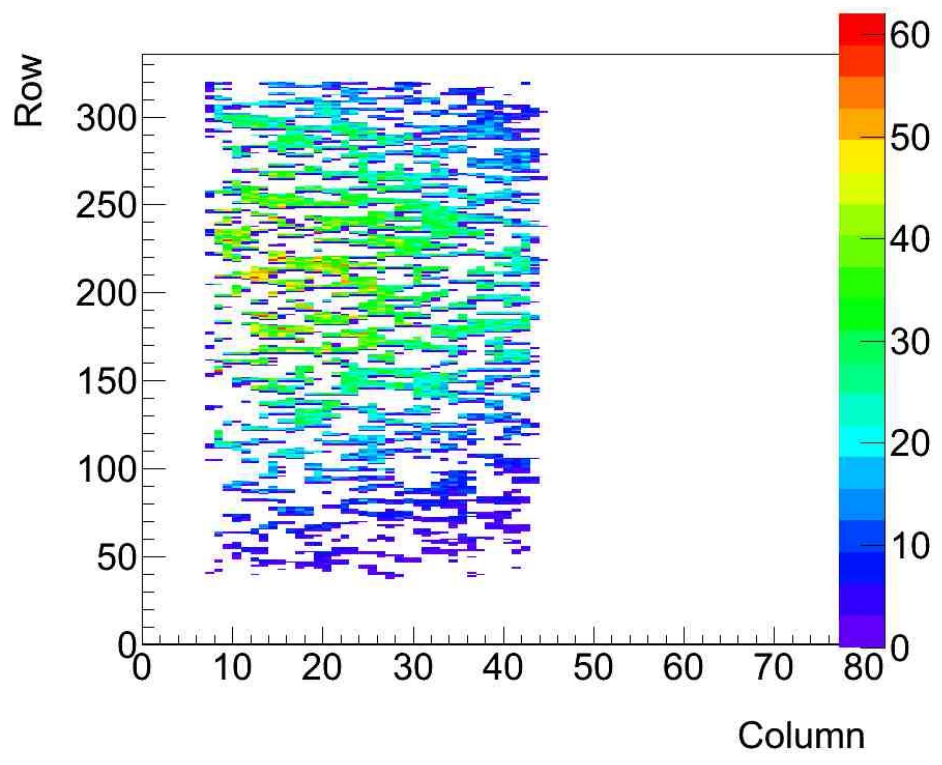

Figure 6.24: Track map of the investigated device, which displays the incident positions of tracks that were matched in a reference plane and in the investigated device. This example of a track map shows the effect of having a device with a large number of noisy pixels, which are visible as white spots in the histogram.

histogram as shown in figure 6.25 , since it shows how many tracks are used to calculate the efficiency in a certain region of a device.

In the efficiency histograms shown in figure 6.25 , the calculated efficiency of a device is plotted against the extrapolated track position on the device.
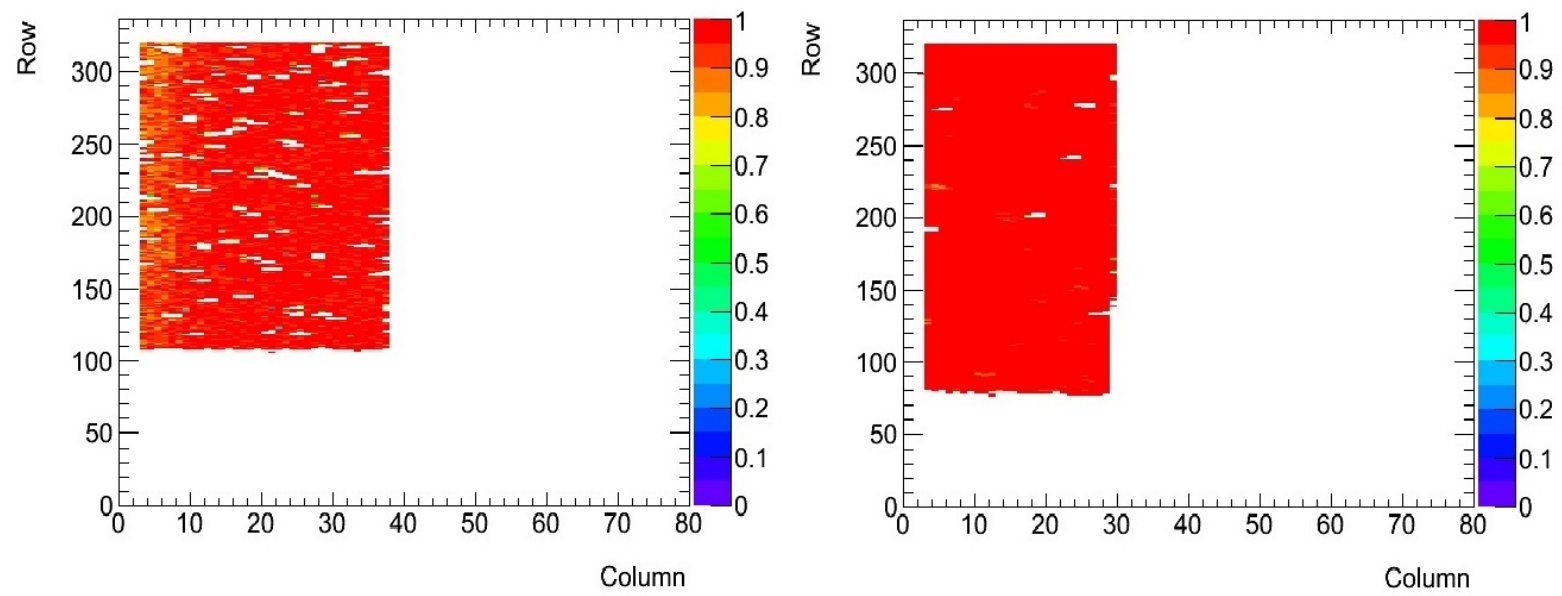

Figure 6.25: The left histogram shows a typical example for a device with a significant number of noisy (masked) pixels and a slight general efficiency drop in the first columns. In the right histogram the efficiency of a device is shown, which was used as reference plane. Only a few pixels were masked and the efficiency homogeneous and high over the device. 


\section{QEfficiency}

The QEfficiency processor is used to histogram the charge collection efficiency of the investigated device. Due to a non-homogeneous design of the pixel cells and resulting charge loss effects in certain regions, the pixel cells detect different signal heights depending on the track incident position within the cell. The used cuts for the QEfficiency processor are:

\begin{tabular}{|l|l|}
\hline cut & histogram \\
\hline \hline tracks through central region & in-pixel charge collection map, \\
$\chi^{2}$ cut on tracks & projection of collected charge to long/short pixel side \\
match in reference plane & \\
track does not hit masked pixel & \\
\hline
\end{tabular}

Table 6.4: QEfficiency: overview of the available cuts and produced histograms.

To visualise the effect of pixel cell features like the bias dot, the QEfficiency processor creates histograms like the one shown in figure 6.26. Here the average detected charge in units of ToT is shown against the track position, projected into one pixel cell. For the projection, the single track positions are taken modulo the pixel pitches in $\mathrm{x}$-/y-direction, such that all information can be displayed in a single pixel cell.

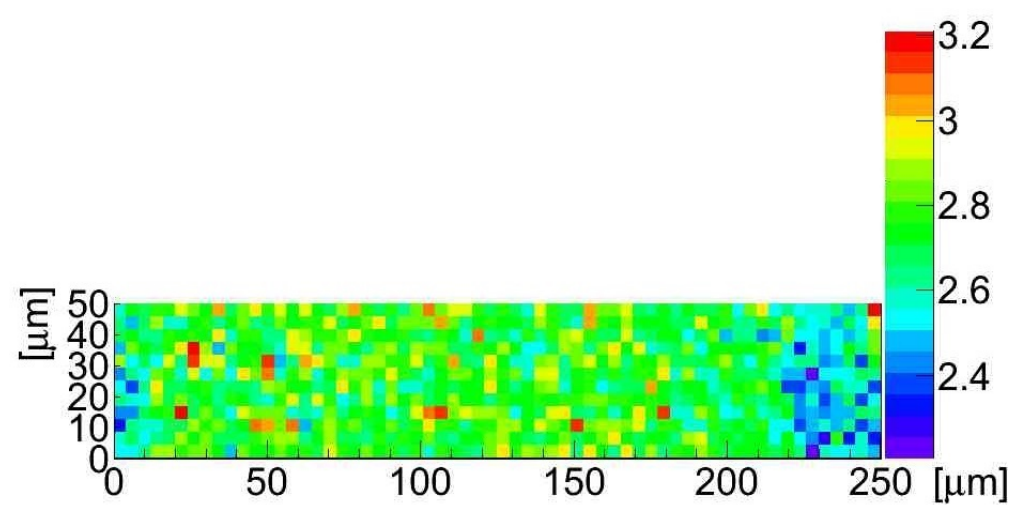

Figure 6.26: Charge collection map for a device with a pixel pitch of $250 \mu \mathrm{m} \times 50 \mu \mathrm{m}$. The average collected charge in units of ToT against the position within a pixel cell is projected into a histogram of a single pixel size. On the right side of the histogram a typical decrease in the collected charge due to the bias dot is visible. Because of the low beam energy used for this measurement (DESY testbeam), the shape of the bias dot is not clearly visible in the histogram.

Figure 6.27 gives a quantitative impression of the charge collection drop in certain regions of the investigated device. The information is projected onto the long side of the pixel cell. The figure shows on the left a typical histogram for a planar silicon pixel sensor, with a slight drop of collected charge on the left edge and a bigger loss on the right in the region of the bias dot. On the right, the figure shows a typical histogram of the same kind for 3D silicon pixel sensors. A regular structure of charge losses can be seen, which appear in the positions of the doped columns. The histograms show different average ToT levels due to different tunings of the devices. 

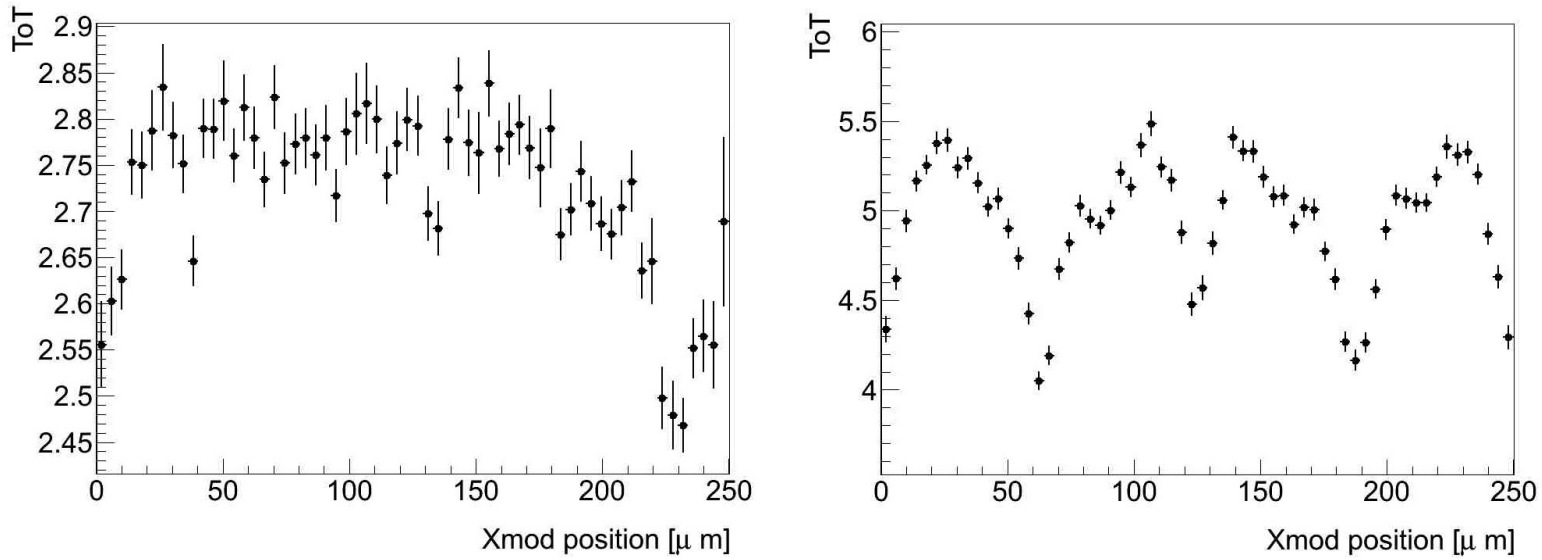

Figure 6.27: The average collected charge of the investigated device is projected on the long side of one pixel with the standard size $250 \mu \mathrm{m} \times 50 \mu \mathrm{m}$. In the left histogram a typical ToT profile of a planar silicon device is shown, the right histogram shows an exemplary histogram of a 3D silicon device.

\section{QShare2D}

When a particle crosses a sensor, a charge cloud starts to drift towards the sensor readout, as described in the introductory chapters. Since this charge cloud has a certain width, cluster sizes larger than one pixel can occur for perpendicular incidence of a particle, because a fraction of the charge cloud can drift to a neighbouring pixel. This means, that the total created charge is shared between the firing pixels. The TBmon QShare2D processor illustrates the probability that charge sharing with the neighbouring pixel occurs, depending on the track incidence point within a pixel cell, see figure 6.28.

For the QShare2D processor the following cuts are used:

\begin{tabular}{|l|l|}
\hline cut & histogram \\
\hline \hline tracks through central region & charge sharing map \\
$\chi^{2}$ cut on tracks & \\
match in reference plane & \\
track does not hit masked pixel & \\
event contains exactly one cluster & \\
cluster size $\leq 4$ & \\
\hline
\end{tabular}

Table 6.5: QShare2D: overview of the available cuts and produced histograms.

The reason for the cut on a cluster size $\leq 4$ is, that the charge sharing between two neighbouring pixels is investigated, where the sharing can occur in $\mathrm{x}$ - and y-direction. Thus, all events containing more hits can be skipped for this kind of analysis.

The width of the region in which charge sharing occurs, allows an estimate of the effective charge cloud width, including threshold effects. A typical charge sharing distribution, projected to an image section of the size of a single pixel and half of the neighbours in each direction, is shown in figure 6.28. The first histogram shows the charge sharing distribution against track incidence point for a planar silicon 
sensor under regular operating conditions. In the central region of the pixel the sharing probability is low, closer to the edge it rises rapidly. The same information can be seen in the second histogram for a 3D silicon sensor under normal operating conditions, giving a higher charge sharing probability around the field electrodes, compared to planar sensors. In the last histogram the results for a planar silicon sensor operated in an under-depleted mode is shown. The charge sharing region is smaller than for full depletion voltage and the sharing probability is lower. This can be explained by the fact that in the situation of shared charge, the neighbouring pixel only gets a fraction of the total charge. In case the sensor is not fully depleted, signal losses occur in the un-depleted region of the sensor. As result, the signal shared with the neighbour pixel is not sufficient to reach the signal threshold, which reduces the total measured charge sharing. Since charge sharing is especially useful to achieve a higher pointing resolution, the width of the sharing region is of high interest for sensor designers.
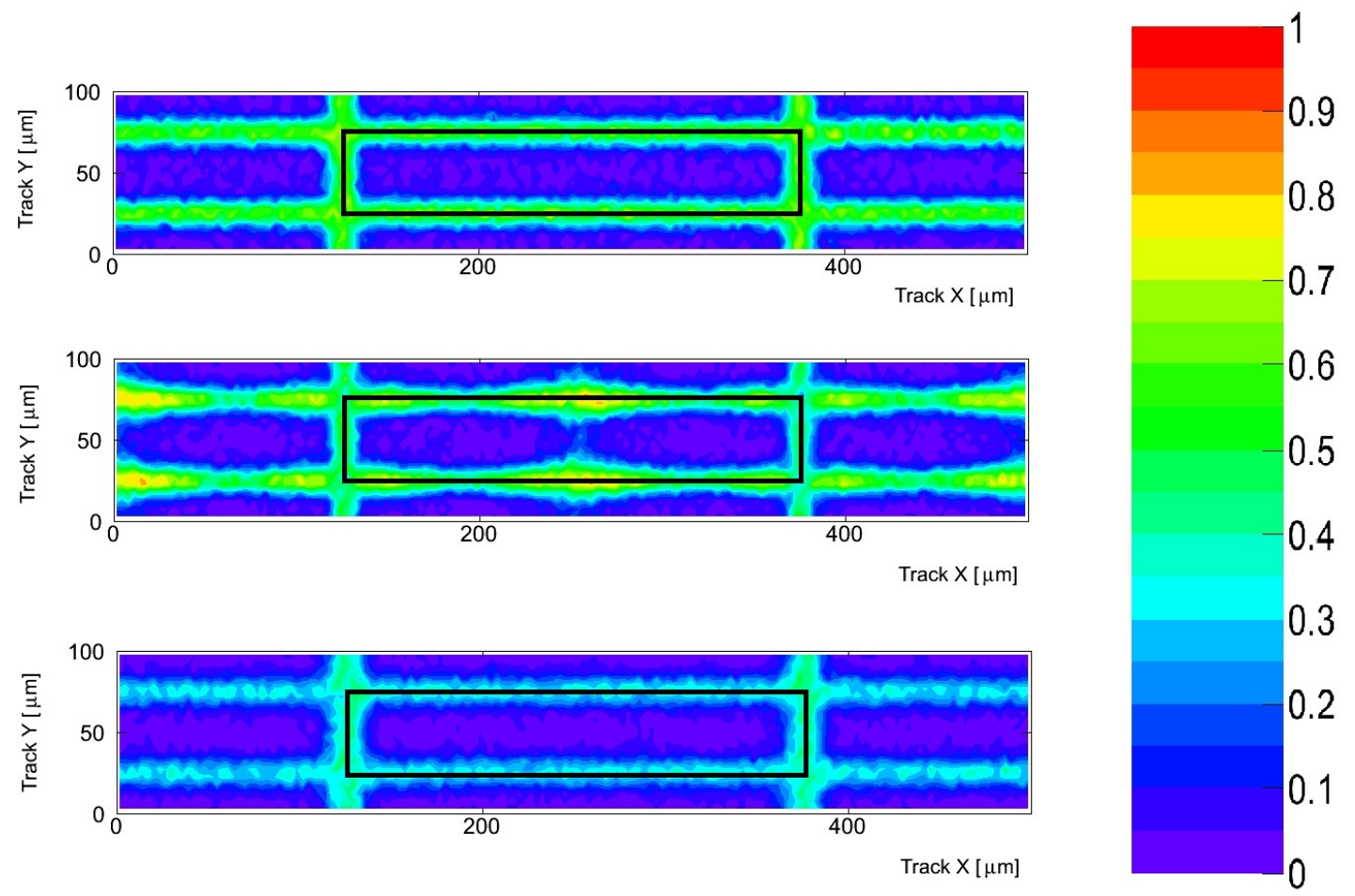

Figure 6.28: Three typical examples for charge sharing maps, where the charge sharing probability depending on the track incidence point is folded into one pixel and half of the neighbouring pixel in each direction. In the center of each histogram, a standard $250 \mu \mathrm{m} \times 50 \mu \mathrm{m}$ pixel cell is indicated by the black frame.

\section{SumToT}

The SumToT processor is used to analyze sensor properties in terms of collected charge. The event/track selection for this processor is done by the following cut flow: 


\begin{tabular}{|l|l|}
\hline cut & histogram \\
\hline \hline tracks through central region & detected charge of matched hits, \\
$\chi^{2}$ cut on tracks & detected summed charge of clusters \\
match in reference plane & \\
track does not hit masked pixel & \\
\hline
\end{tabular}

Table 6.6: SumToT: overview of the available cuts and produced histograms.

The processor histograms per default all charge related information in units of ToT. In case a charge calibration file is available for the investigated device, the calibration is loaded and the conversion from ToT to charge is performed and applied to all histograms.

Figure 6.29 shows the most characteristic histograms of the SumToT processor for an exemplary device. On the left the maximum ToT value per pixel of the matched clusters is histogrammed, showing the expected distribution, as explained in section 3. The distribution shows a small peak for ToT value 14, which is the value the FE-I4 chip sets for charge values exceeding the available range. The sum of the charges detected per matched cluster is shown in the right histogram. Especially in case a ToT calibration was performed prior to the testbeam measurements and the ToT to charge conversion is available, these histograms are of high interest. They allow a direct comparison between expected and detected charge against operating conditions. The device used for this example was tuned to a response of 7 ToT units for a standard MIP-charge of 20,000 $\mathrm{e}^{-}$. Note: for the IBL testbeam measurements described in section 7, the charge calibration functionality for FE-I4 based devices was not available yet.
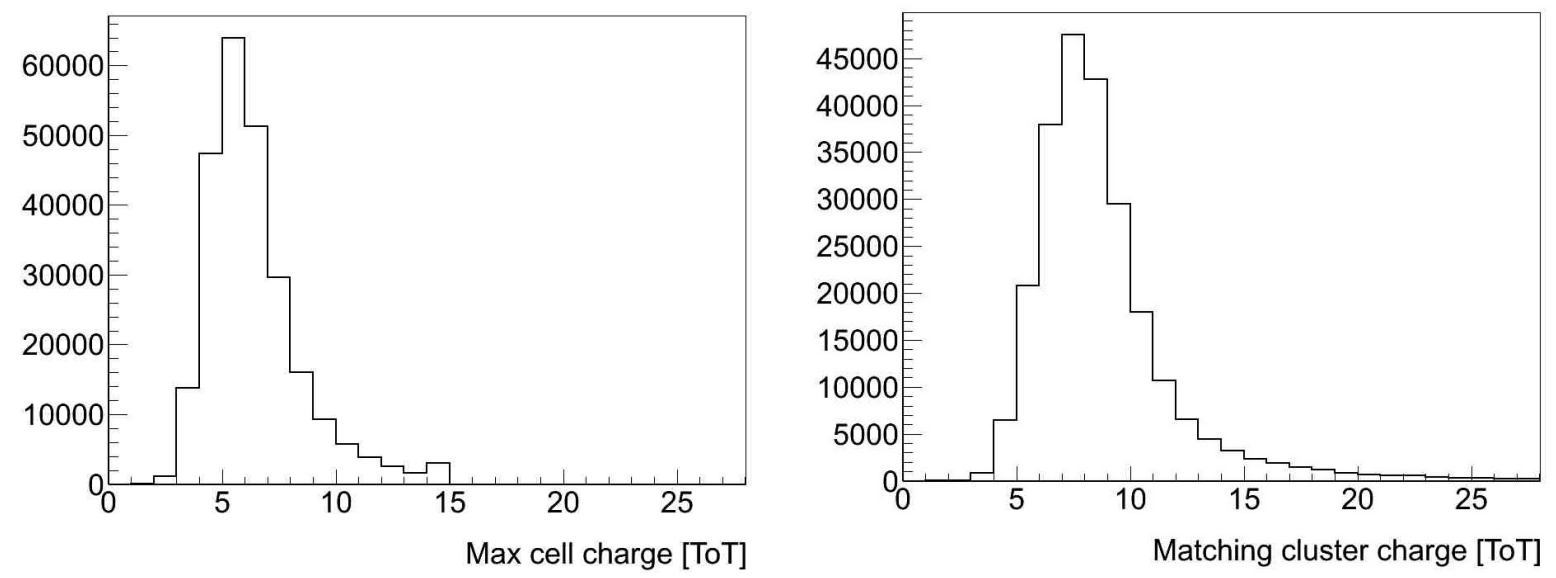

Figure 6.29: Typical output of the SumToT processor, showing on the left the highest charge value of each matched cluster in units of ToT, including the ToT overflow bin at value 14. On the right the charge in units of ToT is histogrammed summed for matched clusters. The device was tuned to a response of 7 ToT units for an injected charge of $20,000 \mathrm{e}^{-}$. 


\subsubsection{Ongoing Developments - TBmon II}

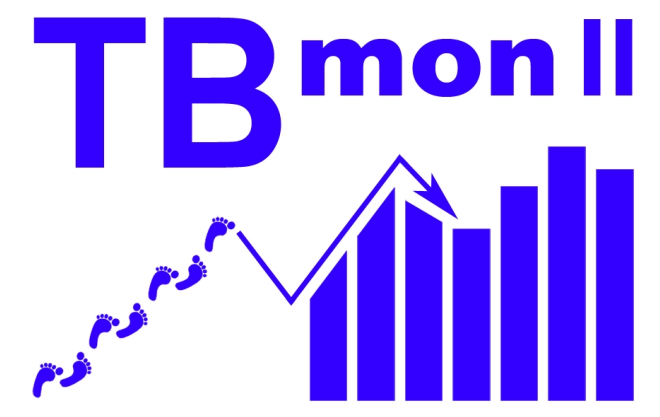

Figure 6.30: TBmonII Logo (designed by Alexander Granget).

The previously described TBmon tool was established as the official analysis tool for all ATLAS pixel related testbeam measurements. Over the years, several analyses processors were added, others were modified and finally the tool contains a large set of processors and in summary it is easy to use. Due to its roots as an online monitor, TBmon has some structural disadvantages. Amongst the most obvious ones there are the analysis configurations, which are part of the software core, and the fact that the data preprocessing is treated as an analysis. The first issue has the consequence that every change in the configurations requires a recompilation of the software, even if just a cut value in an analysis processor was changed or the user decided to load e.g. a hot pixel mask. The second issue has the implication that the preprocessing analyses (HotPixelFinder, CheckAlign, GetEtaCorrection) can not be executed at once, since the analysis processors do not interact with each other. For a good alignment, noisy pixels should be masked, which is only possible in TBmon after recompiling the software, once the hot pixel analysis was performed. The output of each preprocessing step, which is typically dumped into an external file, is only available to the next preprocessing or analysis step after loading and applying the produced file in the subsequent execution of the eventbuilders. This requires each time a change in the configuration files, which necessitates a recompilation of the software. In summary, the user has to execute and recompile TBmon several times, before the actual analyses can be performed.

Furthermore, due to the fact that no central analysis configuration file is available in TBmon, a lot of settings and cut values for analysis processors are hard coded in the processors. Beside that, TBmon offers a very limited geometry description for the devices under test (DUTs), which consists of only one rectangular central pixel type that periodically repeats over the whole defined central sensor region, and one type of rectangular edge pixels. In general, the pixels can only be of rectangular shape and only exactly one "normal" and one "edge" pixel type can be defined.

As final issue in this list, the structure of the event handling in TBmon only allows to process one track per event. Since the typical track multiplicity at DESY and CERN testbeam facilities is around 1.2, this means that around $20 \%$ more data than really necessary has to be recorded, to achieve the target number of events for an analysis.

Due to an increasing number of ATLAS pixel groups performing testbeam analysis and new sensor developments, a new version of TBmon has been developed in collaboration with undergraduate students Daniel Kalin and Konstantin Lehmann, including the outcome of several discussions within the particle physics group at Göttingen University. The new TBmonII version offers a completely new structure, while all analyses developed for the previous version are ported and thus are based on the same calculations. One of the basic structural changes is a new software core, which hosts exclusively the core functionalities. As a consequence, the software just needs to be compiled once after downloading, which 
allows more automation, especially for the data preprocessing. The required manual intervention is reduced to a minimum, lowering the risk of unintentional preprocessing/analysis settings. Once executed, TBmonII creates the output files specified by the user and copies a draft of config files (one analysis config and one general config) into the output directory. These two config files include all available settings and cuts of the whole tool. Examples with comments of an analysis config and a general config file can be found in appendix A.1. Amongst the biggest changes in TBmonII is the new geometry description of devices. While in TBmon only one general pixel type and one edge type, as it was the case for standard FE-I3 and FE-I4 based sensors, were allowed (see figure 6.31), TBmonII offers substantial DUT configuration files for each DUT individually.

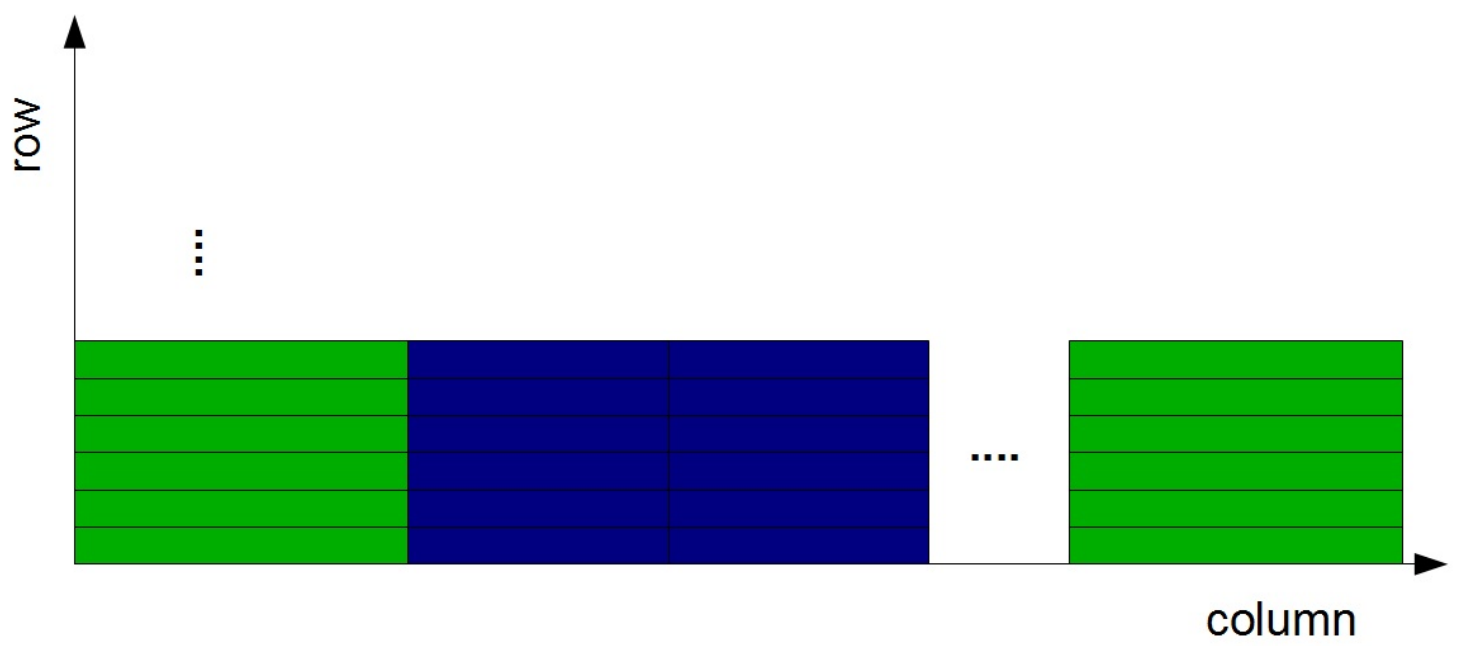

Figure 6.31: Schematic drawing of a typical FE-I4 chip based sensor design, as it is modelled in TBmon and TBmonII. The green rectangles represent the edge pixels, the blue rectangles the regular pixels with a dimension of $250 \mu \mathrm{m} \times 50 \mu \mathrm{m}$.

Here, each DUT configuration can contain an unlimited number of different pixel types, where the pixel types can be modelled out of several substructures, can be repeated in different periodicities and can have different initial coordinates. This allows a proper description of several already existing sensor types, which could not be analysed with the previous version of TBmon. All of the following sensor design examples already exist in reality, while non of them can be analysed without the improvements of TBmonII.

Figure 6.32 shows the schematic drawing of a double chip design, as it is used in the current IBL construction. Although the pixels are arranged in a simple periodic structure, the proper description of such devices was not possible before, since it contains three different pixel types. Another sensor design under investigation, especially by the planar pixel sensor community, are four-chip modules, covering the size of four FE-I4 readout chips. These sensors are of big interest for developments towards future upgrades, as described in section 4.1.2. An exemplary design of a four-chip module is shown in figure 6.33. In addition to the requirements of the double chip design for the geometry description, the four chip sensor has an inactive region. The reason for this inactive part is that the four chip module is simply realized as two double chip sensors on one wafer, which are not cut. Thus, the cutting edge still exists between the upper and lower half of the sensor. Such empty regions can easily be described by TBmonII. Two final examples of designs that can be described, using the new geometry description of TBmonII, are shown in figure 6.34 and 6.35. The first example shows a staggered pixel design, where 


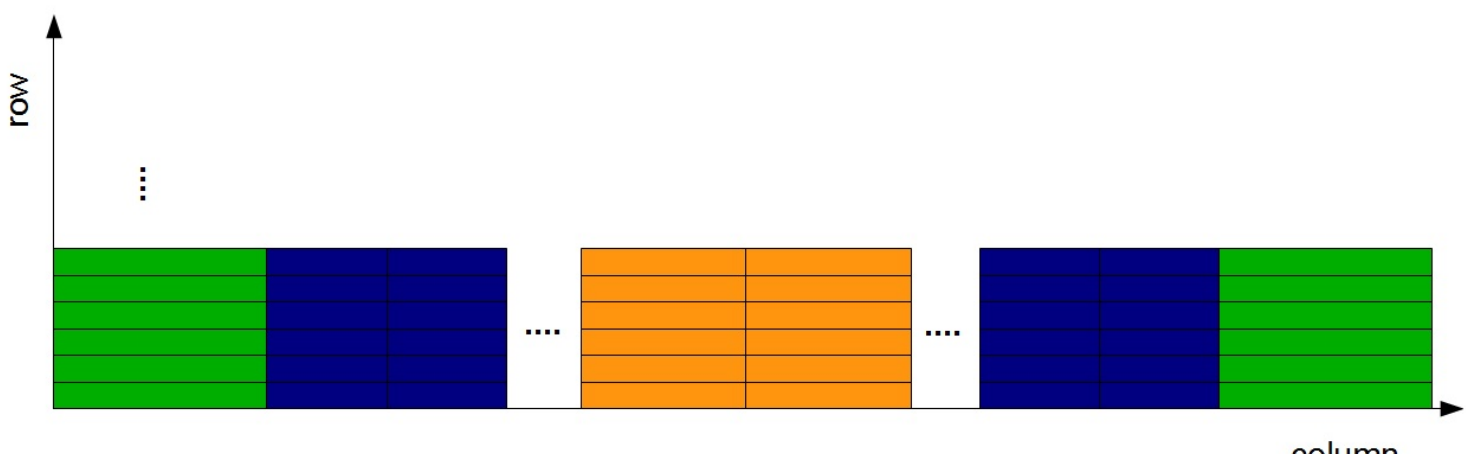

Figure 6.32: Schematic drawing of a FE-I4 double chip sensor, as it is modelled in TBmonII. Again the green rectangles model the edge pixels and the blue rectangles the central pixels. On top of that the central double column (orange), having pixels with a pitch of $450 \mu \mathrm{m} \times 50 \mu \mathrm{m}$, can be included correctly.

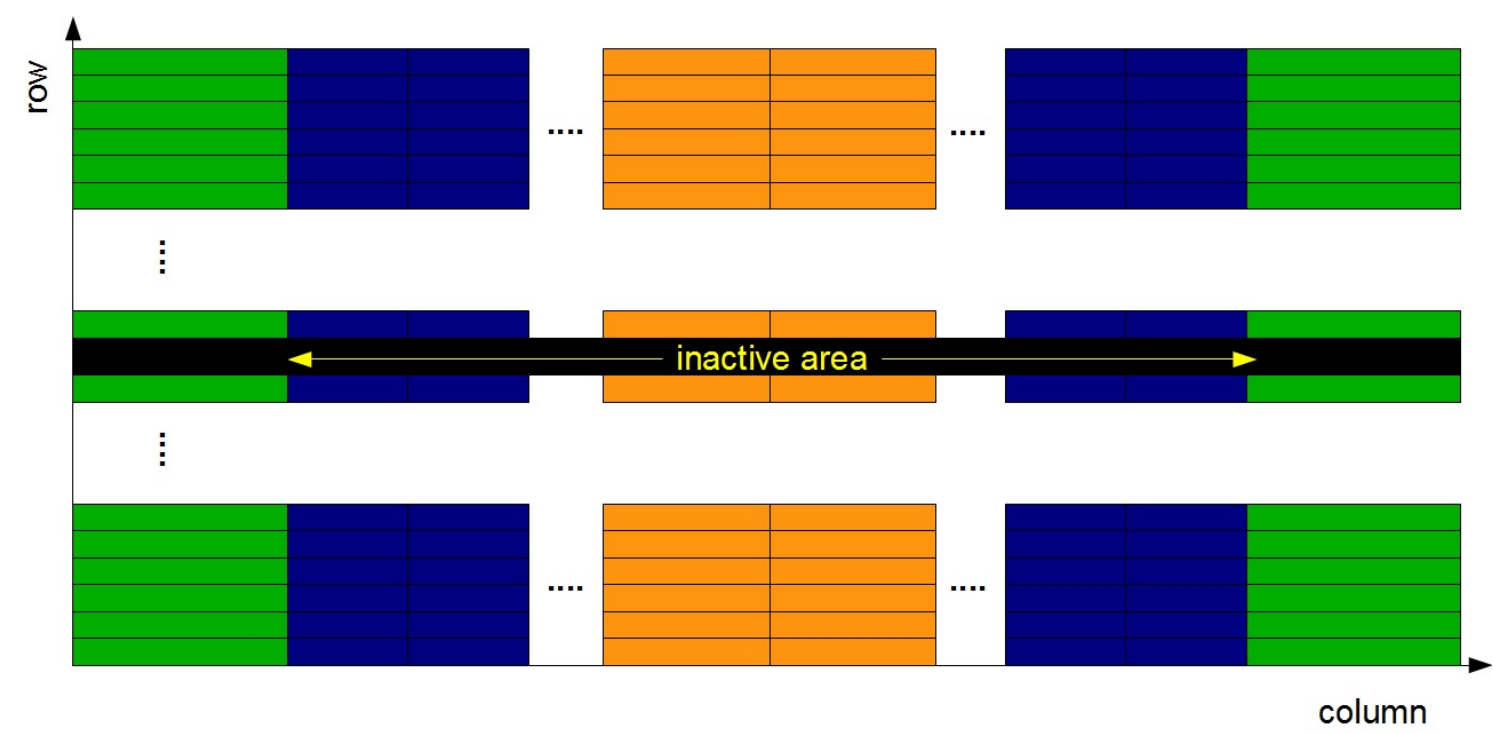

Figure 6.33: Schematic drawing of a FE-I4 four chip sensor, as it can be described in TBmonII. The colour code is the same as in figure 6.32, but additionally an inactive region can be included in the description.

every second row has a different initial coordinate. Since each pixel type can be assigned its own initial coordinate, the proper description of this design type is covered. The second example shows a possible design of L-shaped pixel implants. Since each pixel type can be described by several substructures, such L-shapes or any other pixel design, based on a rectangular substructure, can be described.

Making use of the fact that each DUT has its own geometry description file, that can be specified in the general settings, also individual matching radii can be specified per DUT. Especially for data sets taken at DESY (typical beam energy $4 \mathrm{GeV}$ ), adapted matching radii depending on the position of the device are useful to cover multiple scattering effects. First tests showed an increase in the number of accepted matched tracks due to the proper device description and individual matching radii on the order of $30-40 \%$. Moreover, TBmonII uses a new event handling, which does not only process one track match per event anymore, but investigates all tracks per event. This feature provides an additional increase in the number of usable tracks per run. Results of double chip modules and a four chip module, 


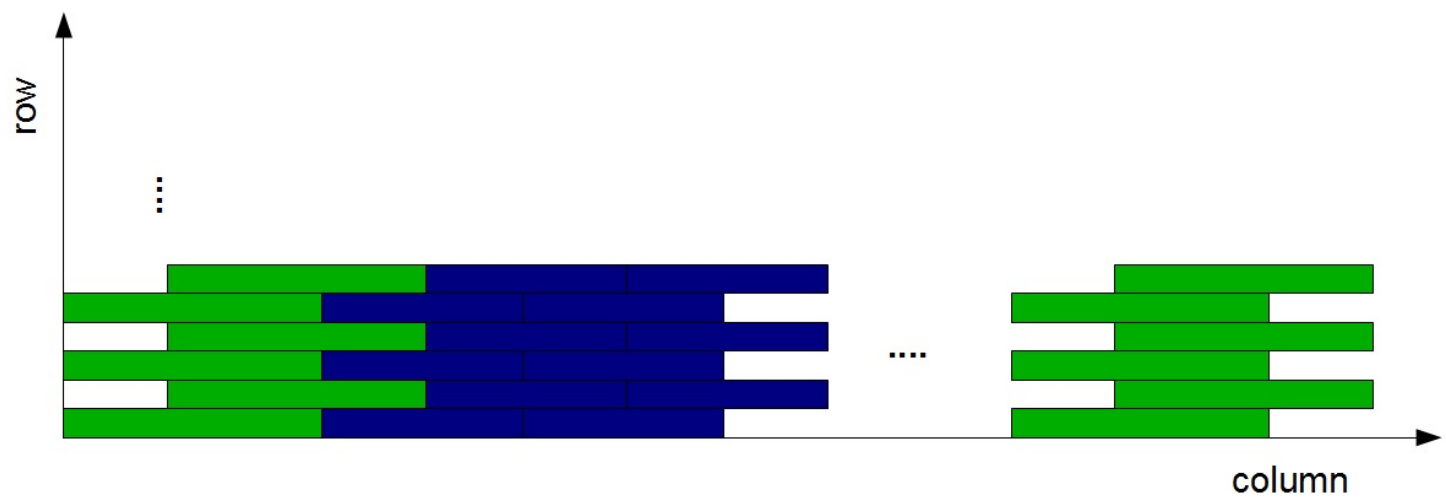

Figure 6.34: Staggered pixel sensor designs, as the one in this schematic, can be described in TBmonII. The geometry description allows different initial coordinates for each geometry, which makes a proper description of the staggered design possible.

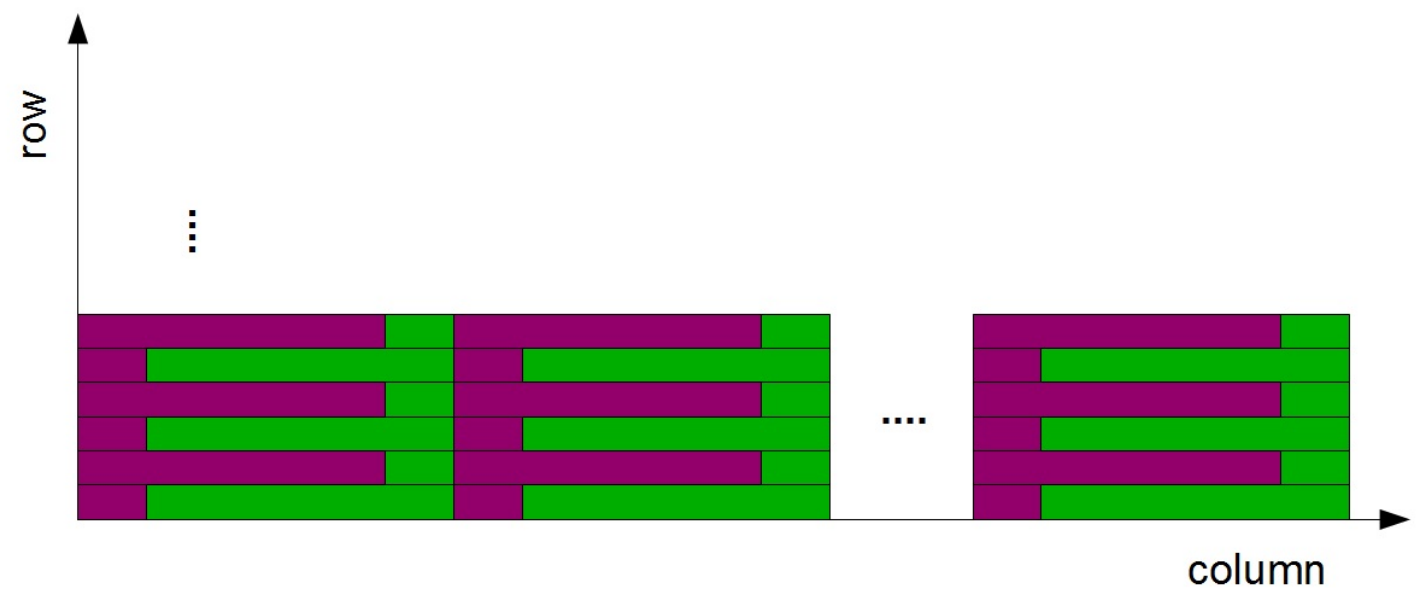

Figure 6.35: As shown in the drawing, TBmonII offers the possibility to model pixels with non-rectangular shapes. Here L-shaped pixels are modelled by combining two rectangular substructures to one L-shaped pixel type. Each pixel type can be described by a combination of several of these substructures.

produced with TBmonII, can be found in the following testbeam analysis section 7 . 



\section{Chapter 7}

\section{Testbeam Analysis}

\subsection{IBL Device Analysis}

In the context of the measurement campaigns investigating new sensor concepts, a set of dedicated IBL testbeam campaigns were performed at CERN (Geneva) and DESY (Hamburg). For all measurements, the EUDET telescope or one of its copies was used, the track reconstruction was performed within the EUTelescope framework and all analyses were done using TBmon. A detailed description of the setup used can be found in section 5, the descriptions of the EUTelescope framework and TBmon are in section 6. Furthermore, a much larger set of exclusive testbeam campaigns of the 3D silicon sensor community and the planar sensor community were performed, testing IBL design sensors and prototypes for future upgrades. Due to several different problems which appeared during IBL testbeam data taking, only a small fraction of the dataset gave reliable results. Out of these results, analyses for each IBL candidate design are presented, which are consistent with the measurements done in the exclusive sensor group testbeams. The latter results are presented in separate publications.

During the IBL testbeam campaigns the focus was on the performance of both types of 3D sensors (double sided and full 3D) and on planar slim edge (SE) sensors, as all three of them are new sensor concepts with respect to the current ATLAS pixel detector. Of special interest were the hit efficiencies of the devices after irradiation as a function of bias voltage and the edge pixel efficiencies of planar SE devices. To investigate these properties, the extrapolated positions on the devices under test (DUTs) of the reconstructed tracks were processed in TBmon using a common set of parameters. Most of the parameters were already described in the TBmon section, additionally an occupancy cut of $5 \cdot 10^{-4}$ was used to identify noisy pixels in the preprocessing steps of TBmon. As match criterion a track matching distance of $400 \mu \mathrm{m}$ in the long pixel direction and $150 \mu \mathrm{m}$ in the short pixel direction was used. All other cuts were set to the default IBL style values, described in the testbeam analysis software section 6.2.

\section{2 n-in-p Sensors}

In 2011, the decision was taken to shorten the IBL development schedule by several years, in order to install the new detector layer during the long shutdown 1 . Thus, the planar pixel community decided to concentrate on the development of $n$-in- $n$ conservative sensors and slim-edge sensors as IBL candidate designs. In parallel, planar $n$-in-p sensors were investigated as potential design candidates for further upgrades. The $n$-in-p design is based on an $\mathrm{n}^{+}$-type readout electrode in $\mathrm{p}$-type bulk material, which has advantages in terms of fabrication steps during sensor production, allowing a single-sided production. For investigations within the ATLAS planar pixel community, the MPP group ${ }^{1}$ and the KEK group ${ }^{2}$

\footnotetext{
${ }^{1}$ www.mpp.mpg.de

${ }^{2}$ www.kek.jp
} 
manufactured different n-in-p sensor designs together with $\mathrm{CiS}^{3}$ and $\mathrm{HPK}^{4}$. First testbeam results, obtained during different planar pixel community testbeam campaigns, are presented in $[72,73]$.

\subsection{IBL Analysis}

The presented IBL testbeam campaigns in the years 2011 and 2012 were organized in mixed batches of two devices at DESY testbeams and four devices at CERN testbeams. Due to the necessity of having a reference device for in-time measurements, one unirradiated device, which passed the quality criteria of lab measurements, was installed in each batch. Typically the operating conditions for the reference device were kept constant throughout a whole measurement batch, except for the temperature.

Particularly, the irradiated sensors required cooling down to less than $-15^{\circ} \mathrm{C}$, which goes in line with the IBL conditions. For the IBL testbeam campaigns, the cooling was realized using blocks of dry ice, which kept the sensors between $-15^{\circ} \mathrm{C}$ and $40^{\circ} \mathrm{C}$, depending on the amount of dry ice left in the polystyrene box (described in section 5.1.2). The resulting uncontrolled change in temperature is an unwanted effect, since it has an impact on the leakage current of the sensors, which again influences the hit efficiency of the devices. Thus, the leakage currents are listed in tables below the results of the efficiency analyses, if available. Note that no automatic, but only a manual temperature and leakage current monitoring was performed during the IBL testbeams. Therefore, the noted values should be taken as rough estimates. Because of the large influence of leakage currents on the hit efficiencies, the systematic error on the hit efficiency values can not be calculated. A rough estimate is a systematic error of approximately $\pm 1 \%$, based on the efficiency spread for the same device at the same conditions, but it was not possible to perform a reliable calculation. Thus, the systematic uncertainties are not included in the following analyses. The error on the applied bias voltage is always estimated to be $\pm 1 \mathrm{~V}$, resulting from the lowest precision high voltage supply, due to a lack of reliable documentation on the usage of high voltage supply channels for the investigated devices. Due to the large number of available tracks per setting, the calculated statistical error is in all cases less than $\pm 0.06 \%$.

Parameters that were varied on purpose are the threshold and feedback tuning, the applied bias voltage and the beam incidence angle. The first two parameters were of big interest in order to investigate whether the tested device can be operated within IBL specifications, while still fulfilling the IBL requirements listed in section 4.1.1. The beam incidence angle was mainly varied between perpendicular incidence, defined as $0^{\circ}$ rotation angle, and a $15^{\circ}$ rotation with respect to perpendicular incidence along the $\phi$-direction (short pixel pitch side). Here the $0^{\circ}$ data corresponds to a "worst case scenario" in terms of signal generation, since out of geometrical reasons the particles have a shorter path through the sensor, than for non-perpendicular incidence. Beside that, local effects caused by the specific sensor design (e.g. doping columns of the 3D sensors) can be observed under perpendicular incidence, since this setting gives the best spatial resolution. In a rotated setup these effects are smeared out over a certain region and the underlying structures can not be identified anymore. In the final detector setup, an incidence angle around $0^{\circ}$ practically does not appear due to the tilt of the staves.

The investigated devices in this section are:

\footnotetext{
${ }^{3}$ Forschungsinstitut für Mikrosensorik und Photovoltaik GmbH, www.cismst.org

${ }^{4}$ Hamamatsu Photonics, www.hamamatsu.com
} 


\begin{tabular}{|l|l|l|l|l|}
\hline identifier & design & thickness $[\mu \mathrm{m}]$ & fluence $\left[10^{15} \frac{\mathrm{n}_{\mathrm{eq}}}{\mathrm{cm}^{2}}\right]$ & irr. type \\
\hline \hline SCC41 & planar conservative & 250 & - & - \\
\hline SCC31 & planar slim edge & 250 & - & - \\
\hline LUB2 & planar slim edge & 250 & 4 & $\mathrm{n}$ \\
\hline SCC40 & planar slim edge & 200 & - & - \\
\hline SCC60 & planar slim edge & 200 & 5 & $\mathrm{p}$ \\
\hline LUB4 & planar slim edge & 200 & 4 & $\mathrm{n}$ \\
\hline PS2 & planar slim edge & 200 & 5 & $\mathrm{p}$ \\
\hline SCC61 & planar slim edge & 200 & $\geq 5$ & $\mathrm{p}$ \\
\hline CNM55 & 3D double sided & 230 & - & - \\
\hline BON_CNM4_3D & 3D double sided & 230 & 5 & $\mathrm{p}$ \\
\hline CNM81 & 3D double sided & 230 & 5 & $\mathrm{n}$ \\
\hline SCC34 & 3D double sided & 230 & 5 & $\mathrm{p}$ \\
\hline SCC82 & 3D double sided & 230 & 5 & $\mathrm{n}$ \\
\hline SCC97 & 3D double sided & 230 & 5 & $\mathrm{p}$ \\
\hline SCC34 & 3D double sided & 230 & 5 & $\mathrm{p}$ \\
\hline FBK13 & full 3D & 230 & - & - \\
\hline SCC87 & full 3D & 230 & 230 & $\mathbf{p}$ \\
\hline SCC90 & full 3D & 230 & 5 & $\mathrm{p}$ \\
\hline FBK11 & & 5 & \\
\hline & & 5 & \\
\hline
\end{tabular}

Table 7.1: Investigated devices of this section. For irradiation fluences specified as $\geq 5 \cdot 10^{15} \frac{\mathrm{n}_{\mathrm{eq}}}{\mathrm{cm}^{2}}$, the radiation monitors in the irradiation facilities did not work properly. Thus, the total gained fluence was higher than demanded, but could not be determined exactly.

The naming scheme for the investigated devices is:

- $\mathrm{SCC}=$ single chip card

- $\mathrm{CNM}=$ Centro Nacional de Microelectronica, vendor of double sided 3D sensors

- FBK = Fondazione Bruno Kessler, vendor of full 3D sensors

- LUB = devices irradiated in Ljubljana (neutron irradiation)

- $\mathrm{PS}=$ device irradiated at CERN PS 


\subsection{Planar Pixel Sensors - Results}

As described in section 4.2, the ATLAS planar pixel community proposed different sensor designs as candidates for the IBL project, based on the design used in the current ATLAS pixel detector. The designs all have in common that they are $\mathrm{n}^{+}$-in-n double chip sensors, which means that each sensor has a size of $41.3 \times 16.8 \mathrm{~mm}^{2}$ and is read out by two FE-I4 chips. Thus, the standard pixel size for the planar devices is $250 \times 50 \mu \mathrm{m}^{2}$, resulting in 160 columns and 336 rows of pixels per double chip sensor. Because the second half of a double chip module is just a mirrored copy of the first half, all tested devices were limited to the size of one FE-I4 chip, except for the very last IBL (production module) testbeam in October 2012. The basic differences in the design proposals by the planar sensor community can be grouped into two approaches, illustrated in figure 7.1.

The first approach is a $250 \mu \mathrm{m}$ thick sensor, equipped with a uniform pixel size of $250 \times 50 \mu \mathrm{m}^{2}$

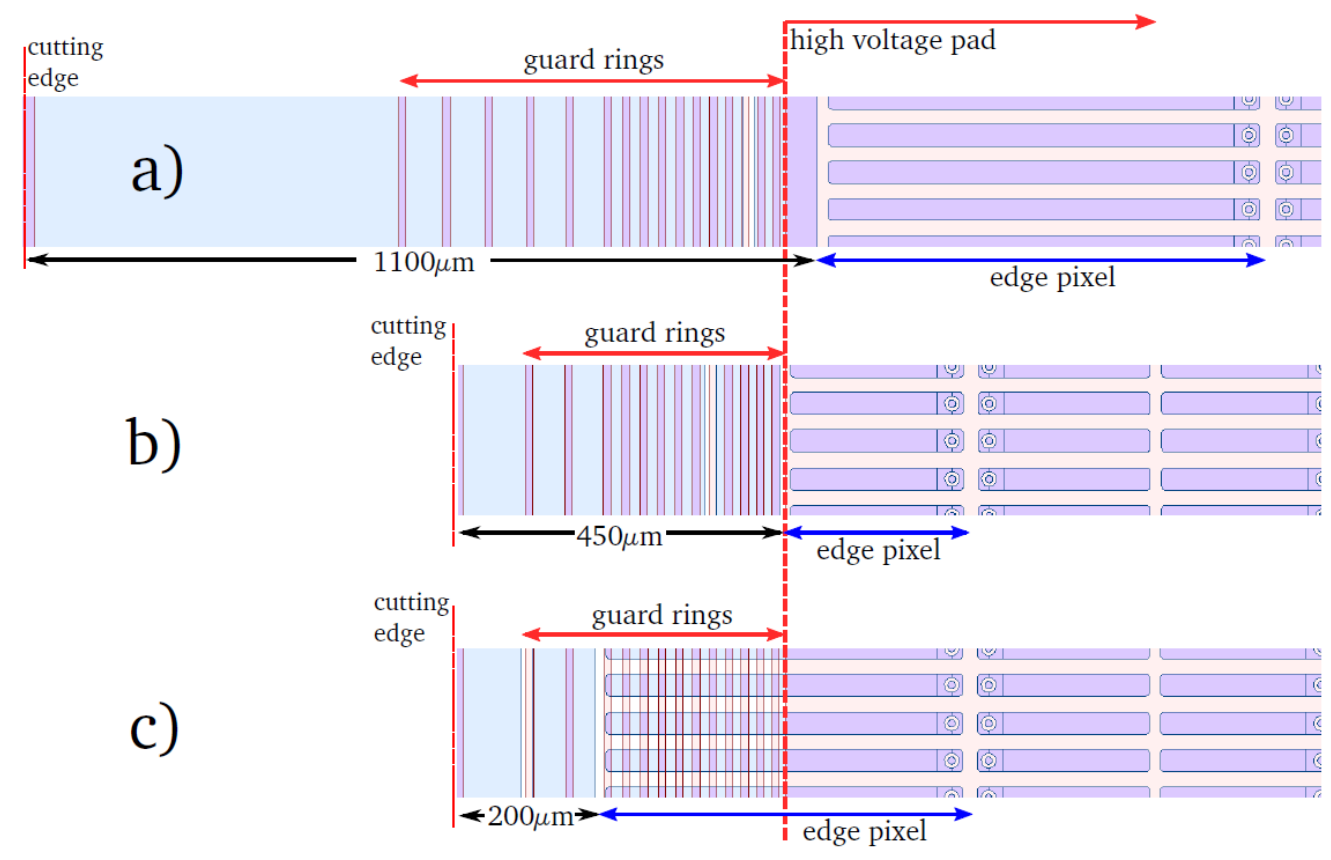

Figure 7.1: Sensor edge designs for different planar pixel sensors. Figure a) shows the sensor edge design of the current ATLAS pixel sensors, with a total edge width of $1100 \mu \mathrm{m}$. Figure b) shows the conservative IBL sensor design with a reduced edge width of $450 \mu \mathrm{m}$, due to less guard rings and smaller safety margins. Finally, figure c) shows the slim edge design, with guard rings shifted on top of the edge pixel, resulting in an edge width of $200 \mu \mathrm{m}[39]$.

throughout the whole sensor. The design included a $450 \mu \mathrm{m}$ wide edge, hosting the guard rings. This design is called "conservative design", since it is closest to the current ATLAS pixel sensor design and has the largest safety margins. Due to the very similar design of the conservative sensors and the current ATLAS pixel sensors, the measurements of main interest in testbeam campaigns were the average hit efficiency and the efficiency of the edge pixels. Figure 7.2 shows that, as expected, the conservative design sensor SCC41 already fulfils the IBL requirements in terms of hit efficiency at a threshold of $3200 \mathrm{e}^{-}$, perpendicular beam incidence and a low bias voltage of $60 \mathrm{~V}$, with a hit efficiency of $99.4 \%$. Increasing the bias voltage to $100 \mathrm{~V}$ increases the average hit efficiency even further to $99.75 \%$, since the higher electric field lowers the probability of trapping. A measurement in the $1.56 \mathrm{~T}$ magnetic field of the Morpurgo Magnet [74] at the CERN H8 beamline showed at slight further increase in average hit 


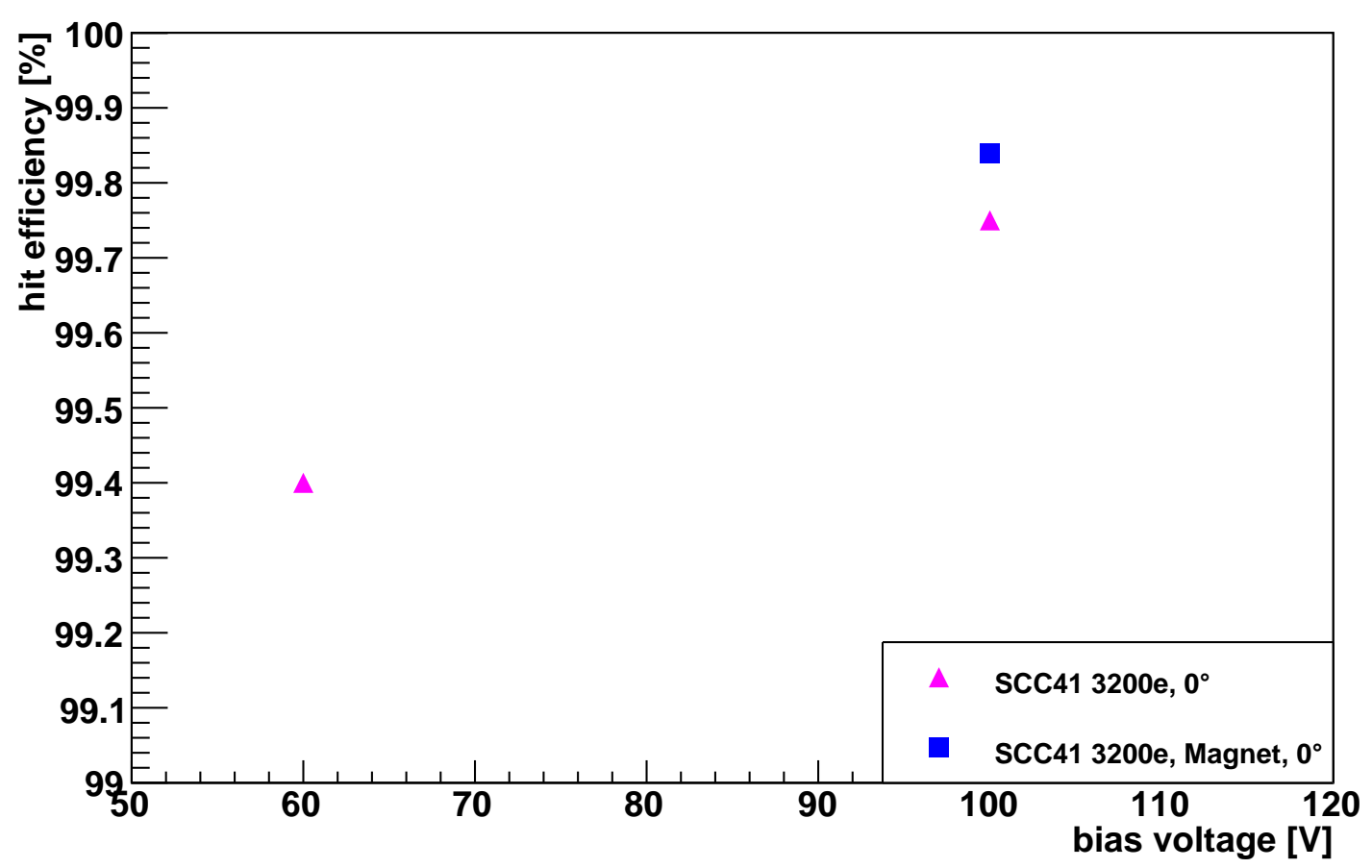

Figure 7.2: Average hit efficiency of device SCC41, which is an unirradiated planar conservative design. Already at moderate bias voltages of $60 \mathrm{~V}$, the sensor fulfils the IBL requirement. Increasing bias voltages or measurements in magnetic fields, increase the hit efficiency even further. Reminder: the calculated statistical error is in all investigated cases less than $\pm 0.06 \%$.

efficiency to $99.84 \%$. This can be explained by the helix shaped particle tracks in magnetic fields, which lead to larger cluster sizes in the tested devices and thus an increased probability to detect the incident particle. For comparison, the mean cluster size of SCC41 at $100 \mathrm{~V}$ bias voltage without B-field is 1.29 , while it increases to 1.78 with B-field.

To illustrate local sensor effects at perpendicular incidence, in-pixel efficiency maps of different types of devices are shown in the following. To ensure sufficient pointing resolution, all presented in-pixel measurements were taken from CERN testbeam data, where the beam energy was $\geq 120 \mathrm{GeV}$. In tables 7.7 and 7.8, the exact operating conditions, characteristics and settings of the devices used for these in-pixel analyses are listed.

In figure 7.3, the in-pixel efficiency for SCC41 is shown under perpendicular incidence. Device SCC41 shows the expected high and homogeneous hit efficiency throughout the whole pixel cell, since it is fully depleted at the used bias voltage. Due to the modified edge pixel design, with respect to the current ATLAS pixel sensors, the hit efficiency of the edge pixels had to be investigated as well. Figure 7.4 shows the measured hit efficiency of the edge pixels, located in the first and last column of device SCC41. The plateau efficiency values are on the same levels as the hit efficiencies for the rest of the device. Since for the conservative design the edge pixels are surrounded by the guard rings, but do not overlap with them, no difference in hit efficiencies between the central region pixels and the edge pixels was expected. The red lines indicate the position of the edge pixel after alignment. For both measurements, with and without magnetic field, the inactive edge width, defined as the distance between the cutting edge and the $50 \%$ efficiency value, is $\approx 445 \mu \mathrm{m}$. 


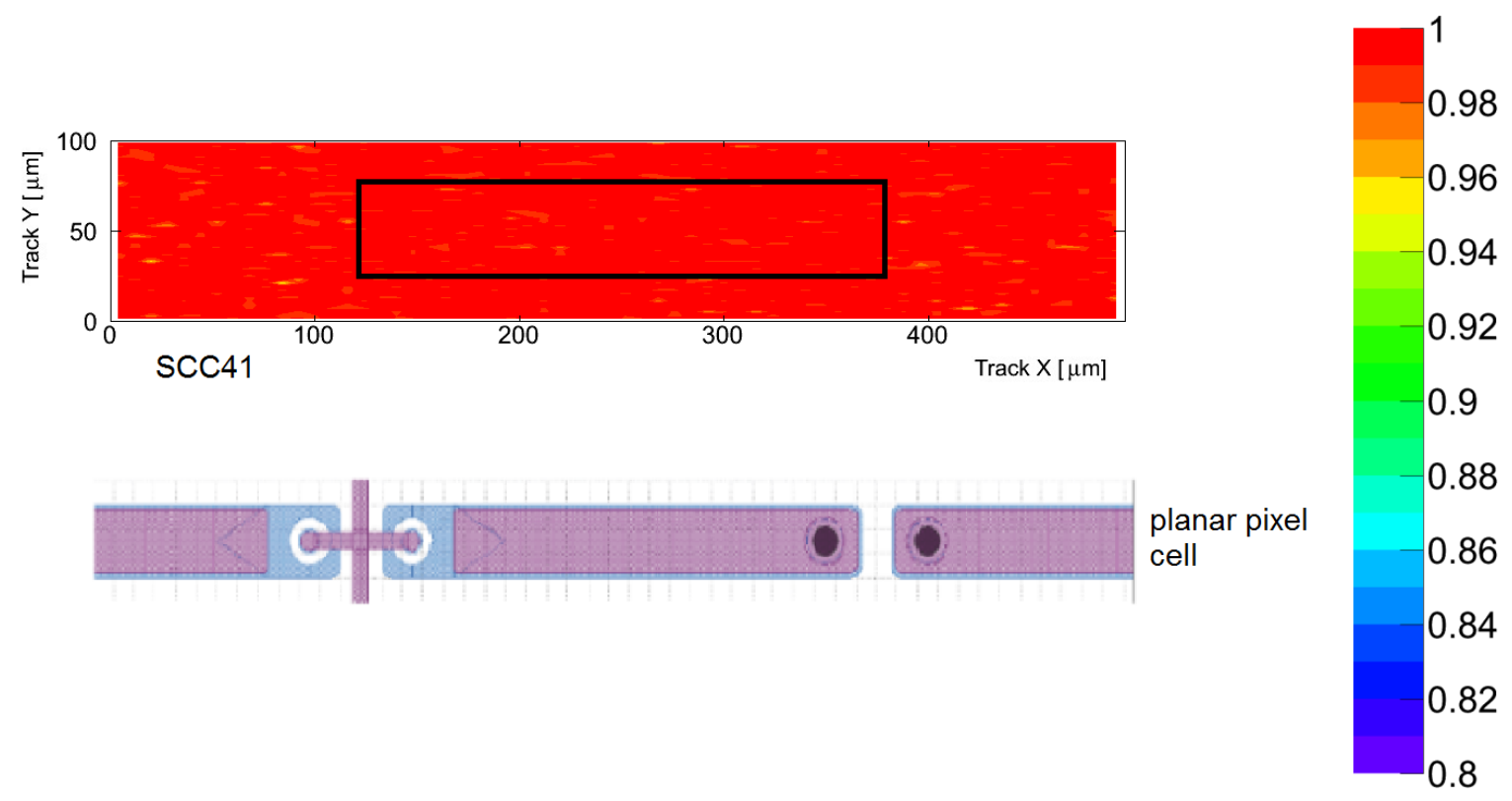

Figure 7.3: In-pixel efficiency for device SCC41 (planar, unirradiated, conservative design) at perpendicular incidence, folded into one pixel and half of its neighbours. The black rectangle in the centre of the histogram frames a standard $250 \times 50 \mu \mathrm{m}^{2}$ pixel. At the bottom, a sketch of the planar pixel cell structure is shown (taken from [44]).
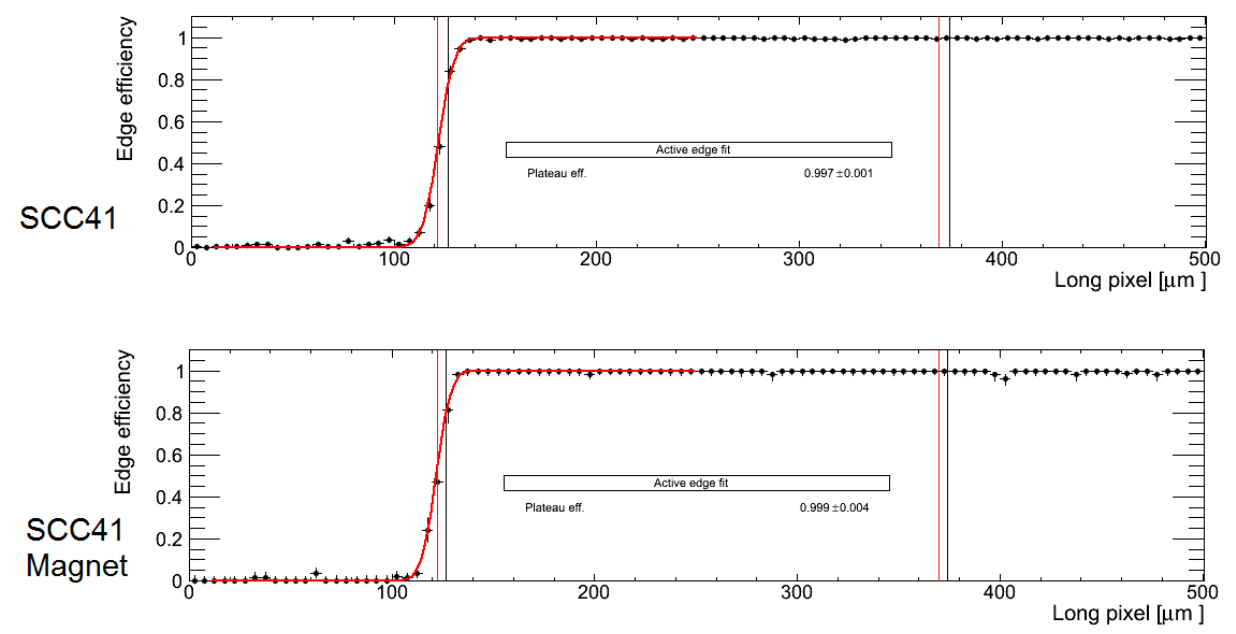

Figure 7.4: Hit efficiency for the edge columns of device SCC41. The histograms show the efficiency in the centre of the histograms, where the vertical black lines indicate a pixel width of $250 \mu \mathrm{m}$ and the red lines mark the position of the edge pixel after alignment. For all edge pixel histograms, the results of the first and last column edge pixels are folded into one histogram. Top: edge pixel efficiency at a bias voltage of $100 \mathrm{~V}, 3200 \mathrm{e}^{-}$threshold and perpendicular incidence. Bottom: edge pixel efficiency at the same settings in magnetic field.

Figure 7.5 shows the distribution of the time over threshold (ToT) values for matched clusters, again for the measurements without and with magnetic field. Consistently, with increasing cluster sizes for measurements in the magnetic field, the collected charge slightly increases, as the particles pass a longer distance in the sensors. Thus, more charge carriers are created and the collected cluster signal increases. The conservative sensor design matched the expectations and especially the IBL requirements, which 

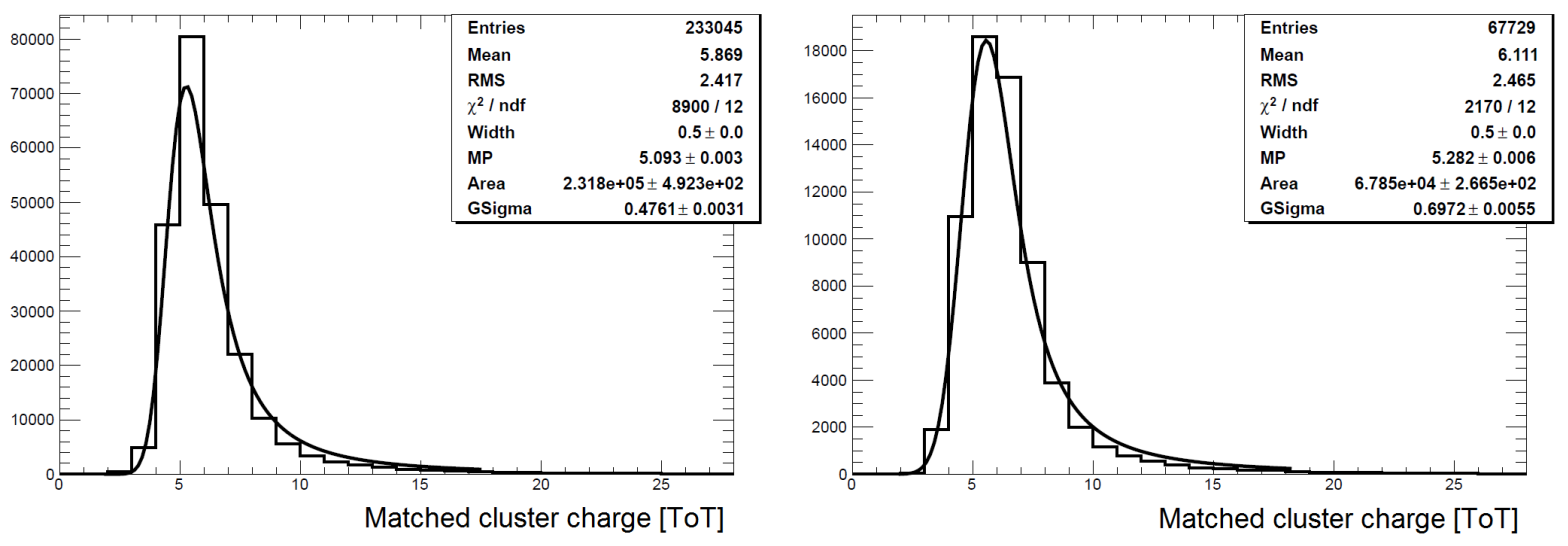

Figure 7.5: Left: ToT distribution of device SCC41 in the measurement without magnetic field. Right: the same measurement in the $1.54 \mathrm{~T}$ field of the Morpurgo magnet. The distribution of the measurement in the magnetic field is slightly shifted towards higher values, which corresponds to the longer particle path through the sensor and the larger cluster sizes. As fit function a convolution of a Gauss and a Landau distribution is used.

was foreseeable due to its similarity to the current ATLAS pixel sensors.

\subsubsection{Slim-Edge Designs}

A more aggressive design of the planar pixel sensor community are the slim edge (SE) sensors. To gain a higher geometric efficiency, the sensor edges are kept as small as possible. This could only be achieved by either shifting the high voltage protection guard rings above the outermost pixels of the sensors or by extending the length of these two pixel columns. During several testbeam campaigns by the planar pixel community, it was investigated whether the edge pixels work at all when shifted underneath the guard rings and it was studied how far they can be shifted [73]. The main concern with this design was, that the electric field in the edge pixels is far from homogeneous, if the guard rings are placed on top of them. Thus, the question was, whether charge can be collected for pixels below guard rings and if yes, how far they can be shifted without loosing too much signal. To understand this point systematically, different sensors with stepwise shifted edge pixels were produced, e.g. the DO3 sensor. This sensor has a thickness of $285 \mu \mathrm{m}$ and as speciality blocks of ten edge pixels, where each of these blocks is shifted $25 \mu \mathrm{m}$ further to the sensor edge, compared to the previous block, as shown in figure 7.6. The testbeam measurements showed, that even for pixels shifted far below the guard rings, sufficient charge can be collected. Moreover, the analysis showed that for the tested structures the length of the active region mainly depends on the distance between the high voltage pads and the outer ends of the edge pixels.

These results lead to the final design of the planar slim edge sensors, with edge pixels of $500 \mu \mathrm{m}$ length, of which $250 \mu \mathrm{m}$ are shifted below the guard rings, see figure 7.1. This reduces the edge width of these slim edge sensors to $200 \mu \mathrm{m}$. The SE sensors were produced in two different variants for the IBL qualification tests, one of them with a sensor thickness of $250 \mu \mathrm{m}$, the other one with a sensor thickness of $200 \mu \mathrm{m}$.

The $250 \mu \mathrm{m}$ thick slim edge sensors were a compromise between the conservative sensor design and the very aggressive $200 \mu \mathrm{m}$ slim edge sensors. Again, the measurement of highest interest for the $250 \mu \mathrm{m}$ SE devices, was the average hit efficiency, which is shown in figure 7.7 and the corresponding conditions in table 7.2.

The unirradiated device SCC31 shows an average hit efficiency of close to $100 \%$, which slightly de- 

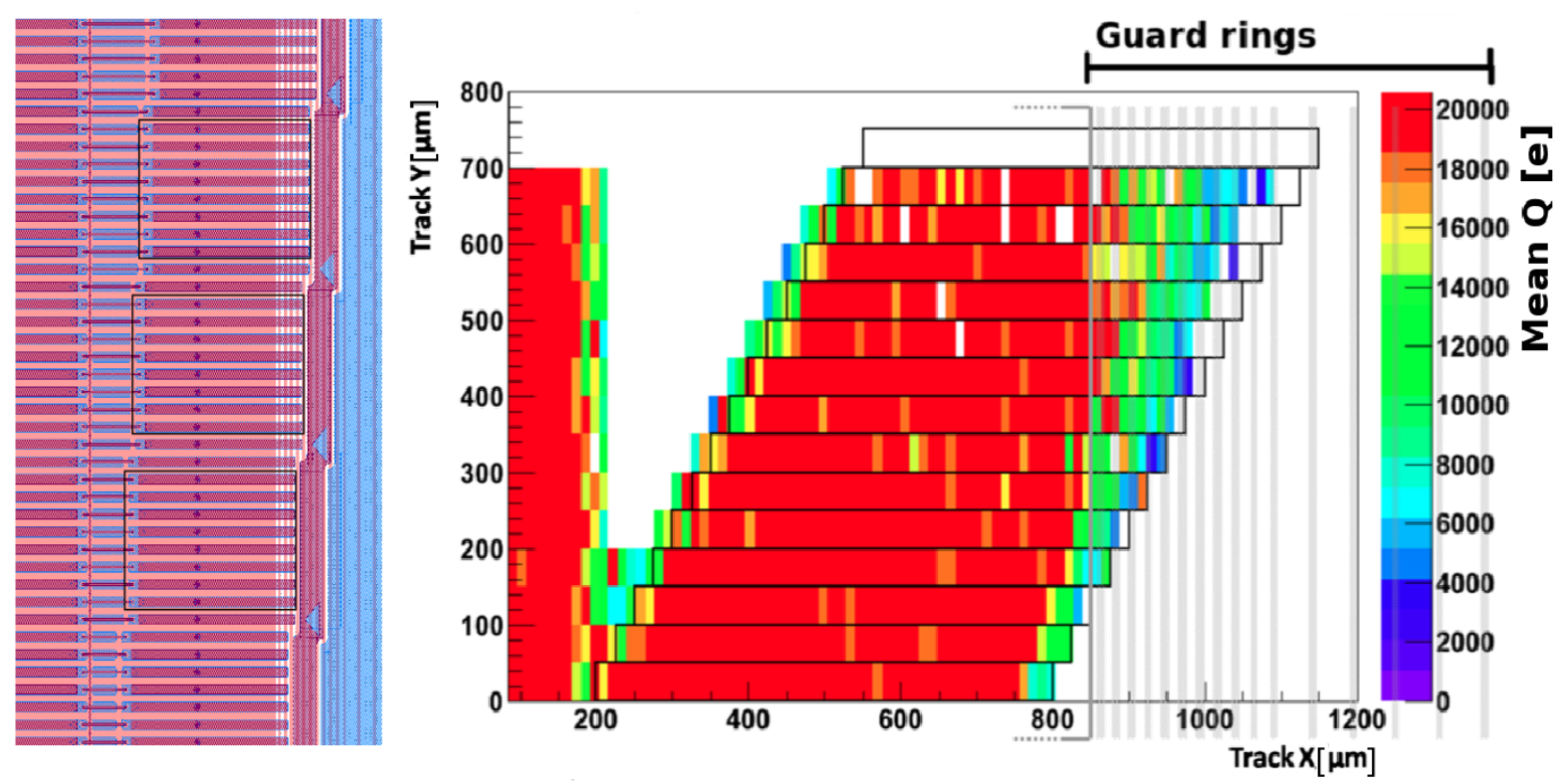

Figure 7.6: Left: structure of DO3 sensor, with blocks of ten edge pixels shifted in steps of $25 \mu \mathrm{m}$ towards the sensor edge. To avoid effects of neighbouring sets in the analysis results, only the innermost eight pixels of each set are used (black frame). Right: collected charge in shifted edge pixels of DO3 at a bias voltage of $100 \mathrm{~V}$. Data of pixels from the same shift-block are folded into the size of one pixel [73].

creases with increasing temperature and thus rising leakage current. Device LUB2 is the same design, but irradiated to a fluence of $4 \cdot 10^{15} \frac{\mathrm{n}_{\mathrm{eq}}}{\mathrm{cm}^{2}}$, which is close to the IBL requirements of $5 \cdot 10^{15} \frac{\mathrm{n}_{\mathrm{eq}}}{\mathrm{cm}^{2}}$. Histogram 7.7 shows that for under-depletion at $400 \mathrm{~V}$ the hit efficiency is on the order of $95 \%$, but increases with increasing depletion voltage until it reaches a plateau at around $800 \mathrm{~V}$. The fact that the hit efficiency drops for depletion voltages below $800 \mathrm{~V}$ is consistent with the measurement of the collected charge per detected cluster, shown in figure 7.8. Although the fit is not perfect for the $600 \mathrm{~V}$ measurement, it can clearly be seen that, the most probable value for the collected charge is far below 5 ToT. Note: since the documentation of the leakage currents for this device has large gaps, no clear conclusions can be drawn concerning the dependency of the hit efficiency and the leakage currents.

One of the most interesting measurements with the LUB2 device was the first measurement of the edge efficiency for an irradiated slim edge device. The results are shown in figure 7.9. The dip in the efficiency plateau on the right of each histogram can be explained by the bias dot of the pixel cells. On the left of the $500 \mu \mathrm{m}$ wide marked region in the histograms, which denotes the long edge pixel, an S-curve fit is applied around the edge region, where edge pixels showing 50\% hit efficiency at the end of pixel cell are defined as fully efficient. The calculated plateau efficiency only takes into account the part of the edge pixels, which is located below the guard rings. For a bias voltage of $1000 \mathrm{~V}$ the edge pixels are fully active and efficient over the full length, as shown in the first histogram. With decreasing bias voltages of $800 \mathrm{~V}$ and $600 \mathrm{~V}$ the edge efficiency drops until finally at $400 \mathrm{~V}$ the hit efficiency of the edge pixels decreases significantly. In the bottom histogram, a further measurement at $1000 \mathrm{~V}$ bias voltage with higher statistics is shown, where the efficiency of the edge pixels is back to the previous plateau value.

For the measurement at $1000 \mathrm{~V}$ bias, the inactive edge has a width of $\approx 196 \mu \mathrm{m}$. During further measurements with SE devices in IBL testbeams at CERN, the edge pixels were either masked or not in the beam. During testbeams at DESY, the beam energy is too low to sufficiently measure such in-pixel 


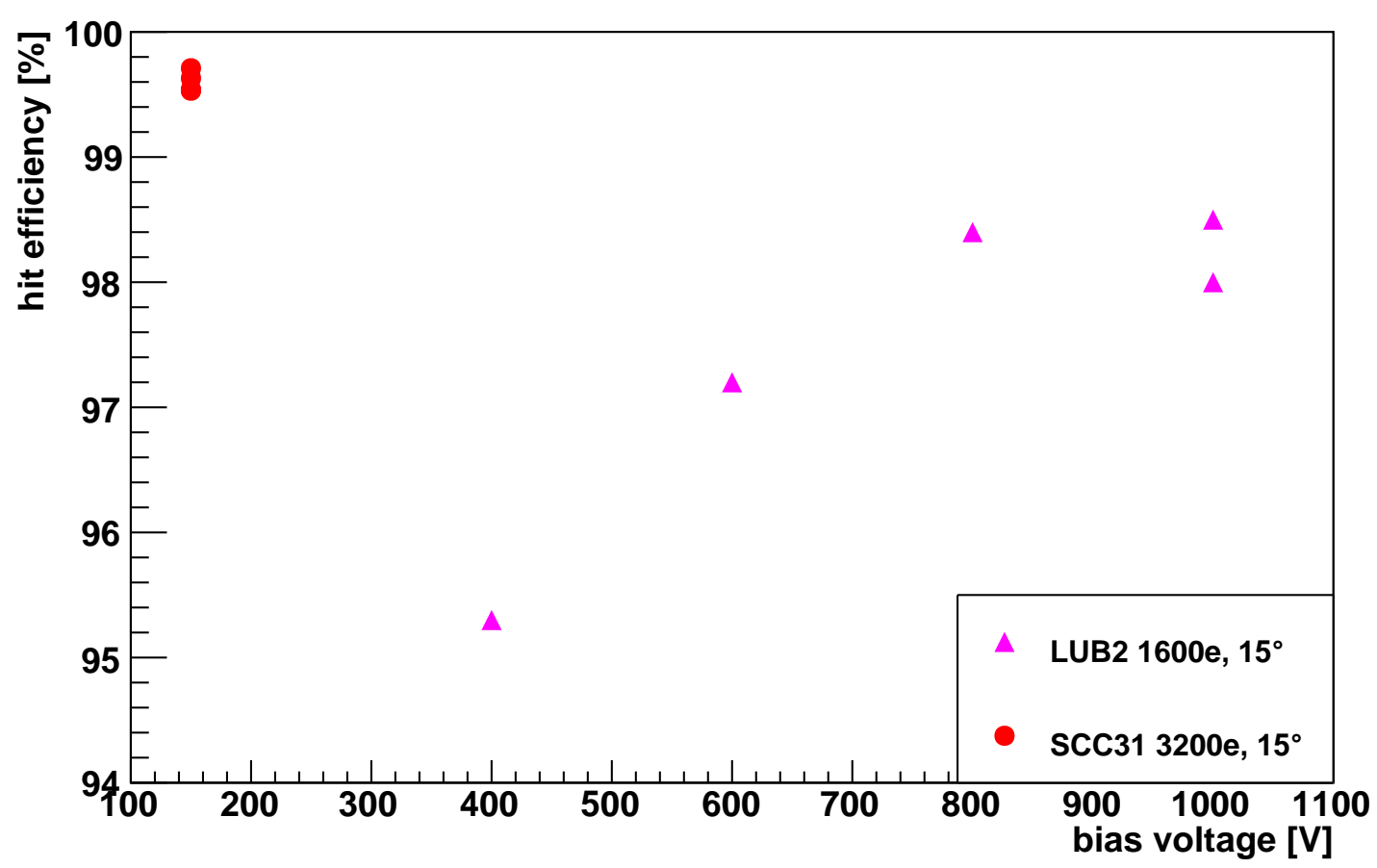

Figure 7.7: Average hit efficiency of $250 \mu \mathrm{m}$ thick planar slim edge sensors. Device SCC31 is an unirradiated reference sensor, while LUB2 is irradiated to $4 \cdot 10^{15} \frac{\mathrm{n}_{\mathrm{eq}}}{\mathrm{cm}^{2}}$. The unirradiated sensor already shows a high efficiency of more than $99.5 \%$ for low bias voltages, the irradiated sensor reaches its plateau efficiency at a bias above $800 \mathrm{~V}$.
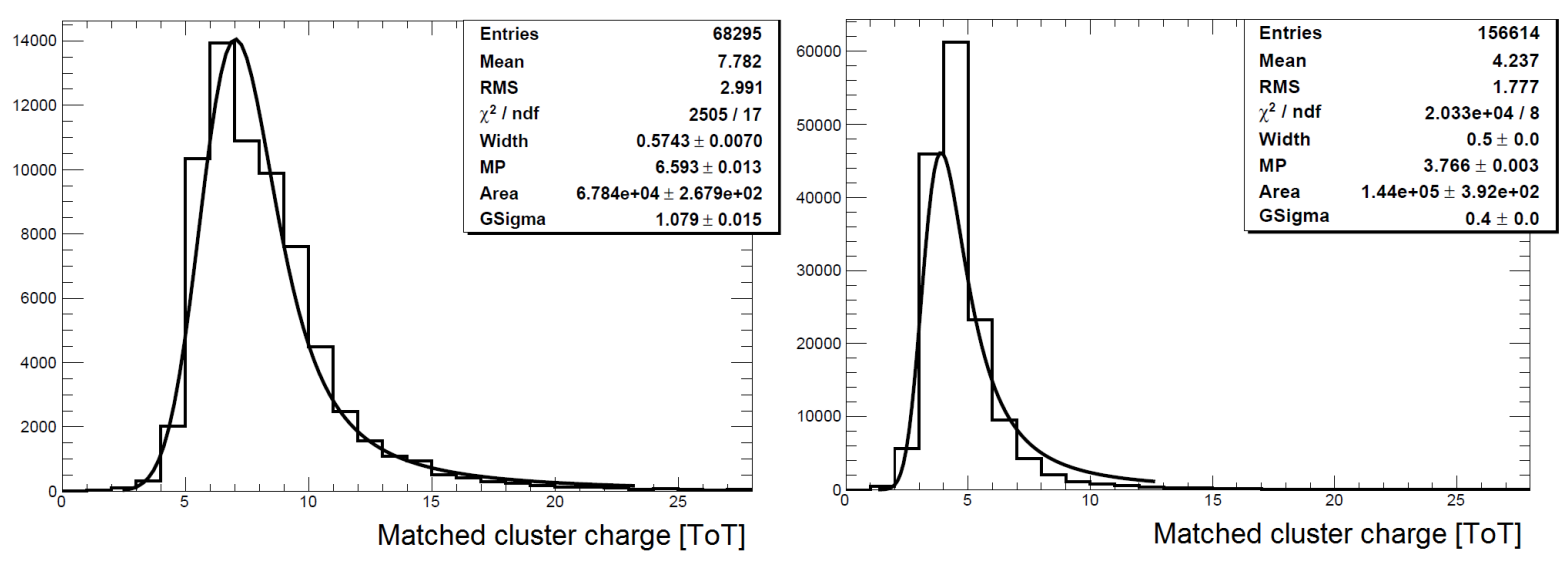

Figure 7.8: Left: ToT distribution of device LUB2 at a bias voltage of $1000 \mathrm{~V}$. Right: the same measurement at a bias voltage of $600 \mathrm{~V}$. The collected charge for the lower voltage measurement is significantly decreased, which is consistent with the lower hit efficiency.

effects, since multiple scattering lowers the pointing resolution drastically. More SE devices were tested during the various testbeam campaigns of the planar pixel community.

Since it was the most aggressive design, $200 \mu \mathrm{m}$ thick slim edge sensors were of highest interest dur- 


\begin{tabular}{|l|c|c|c|c|c|}
\hline sensor ID & bias voltage [V] & $\mathrm{I}_{\text {leak }}[\mu \mathrm{A}]$ & average eff. [\%] & tuning [ToT@ $\left.\mathrm{ke}^{-}\right]$ & threshold $\left[\mathrm{e}^{-}\right]$ \\
\hline \hline SCC31 & 150 & 0.001 & 99.72 & $8 @ 20$ & 3200 \\
\hline & 150 & 0.001 & 99.63 & $8 @ 20$ & 3200 \\
\hline & 150 & 0.002 & 99.54 & $8 @ 20$ & 3200 \\
\hline LUB2 & 150 & 0.002 & 99.53 & $8 @ 20$ & 3200 \\
\hline & 400 & 40 & 95.3 & $5 @ 20$ & 1600 \\
\hline & 600 & 28 & 97.2 & $5 @ 20$ & 1600 \\
\hline & 800 & 96 & 98.4 & $5 @ 20$ & 1600 \\
\hline & 1000 & 65 & 98.5 & $5 @ 20$ & 1600 \\
\hline & 1000 & 73 & 98.0 & $5 @ 20$ & 1600 \\
\hline
\end{tabular}

Table 7.2: Bias voltages, leakage currents, average efficiencies and tunings of planar $250 \mu \mathrm{m}$ thick SE sensors.

\begin{tabular}{|l|c|c|c|c|c|}
\hline sensor ID & bias voltage [V] & $\mathrm{I}_{\text {leak }}[\mu \mathrm{A}]$ & average eff. [\%] & tuning [ToT@ $\mathrm{ke}^{-}$] & threshold $\left[\mathrm{e}^{-}\right.$] \\
\hline \hline SCC40 & 60 & 0.007 & 99.88 & $8 @ 10$ & 1600 \\
\hline & 100 & 0.12 & 99.72 & $8 @ 10$ & 1600 \\
\hline & 150 & - & 99.94 & $8 @ 10$ & 3200 \\
\hline & 150 & - & 99.89 & $8 @ 10$ & 3200 \\
\hline
\end{tabular}

Table 7.3: Bias voltages, leakage currents, average efficiencies and tunings of planar $200 \mu \mathrm{m}$ thick SE sensors. The measurement at $60 \mathrm{~V}$ bias voltage was performed in the magnetic field of the Morpurgo magnet.

ing the IBL qualification measurements. The performance of an unirradiated device was studied using SCC40. This device was investigated for different voltages and in the magnetic field of the Morpurgo magnet, using high statistics. The average hit efficiency for the device under different conditions are shown in figure 7.10 and the corresponding measurement conditions in table 7.3. In-pixel efficiency measurements for planar slim edge devices are shown in figure 7.11.

For the measurement in the magnetic field, the hit efficiency reaches the same values as for high bias voltages. Again, the magnetic field causes the particles to cross more pixels, which can be seen in the increase of the average cluster size from 1.19 at $100 \mathrm{~V}$ bias voltage, compared to 1.85 even at lower bias of $60 \mathrm{~V}$ with B-field.

A larger set of measurements was performed with irradiated $200 \mu \mathrm{m}$ thick SE sensors. An overview of the average hit efficiencies at different settings is shown in figure 7.12. The measurement conditions are summarized in table 7.4, an exemplary in-pixel efficiency plot of device LUB4 can be found in figure 7.11.

Since the operation parameters of the irradiated planar $200 \mu \mathrm{m}$ thick SE device PS2 were varied in terms of threshold and bias voltage, the collected cluster charge in units of ToT at different bias voltages and thresholds is shown in figure 7.13. As the overview shows, the effect on the collected charge is significant when going to slightly higher thresholds, but small compared to the effect of lowering the 


\begin{tabular}{|c|c|c|c|c|c|}
\hline sensor ID & bias voltage $[\mathrm{V}]$ & $\mathrm{I}_{\text {leak }}[\mu \mathrm{A}]$ & average eff. [\%] & tuning [ToT@ $@ \mathrm{ke}^{-}$] & threshold $\left[\mathrm{e}^{-}\right]$ \\
\hline \multirow[t]{4}{*}{ SCC60 } & 550 & - & 92.35 & $8 @ 10$ & 1600 \\
\hline & 550 & 365 & 89.69 & $8 @ 10$ & 1600 \\
\hline & 550 & 500 & 89.54 & $8 @ 10$ & 3200 \\
\hline & 1000 & 1221 & 97.51 & $8 @ 10$ & 3200 \\
\hline \multirow[t]{2}{*}{ LUB4 } & 600 & 15 & 97.16 & $8 @ 10$ & 1600 \\
\hline & 1000 & 125 & 97.85 & $8 @ 10$ & 1600 \\
\hline \multirow[t]{9}{*}{ PS2 } & 600 & 72 & 93.04 & $8 @ 10$ & 1500 \\
\hline & 800 & 88 & 97.63 & $8 @ 10$ & 1500 \\
\hline & 1000 & 145 & 98.35 & $8 @ 10$ & 1500 \\
\hline & 600 & 130 & 87.03 & $8 @ 10$ & 1800 \\
\hline & 800 & 132 & 96.68 & $8 @ 10$ & 1800 \\
\hline & 1000 & $100-140$ & 98.0 & $8 @ 10$ & 1800 \\
\hline & 600 & 140 & 83.31 & $8 @ 10$ & 2000 \\
\hline & 800 & 132 & 96.07 & $8 @ 10$ & 2000 \\
\hline & 1000 & 223 & 97.63 & $8 @ 10$ & 2000 \\
\hline \multirow[t]{5}{*}{ SCC61 } & 600 & 57 & 84.90 & $5 @ 20$ & 1600 \\
\hline & 800 & 230 & 94.39 & $5 @ 20$ & 1600 \\
\hline & 800 & 277 & 93.03 & $5 @ 20$ & 1600 \\
\hline & 1000 & 106 & 95.25 & $5 @ 20$ & 1600 \\
\hline & 1000 & 160 & 96.05 & $5 @ 20$ & 1600 \\
\hline
\end{tabular}

Table 7.4: Bias voltages, leakage currents, average efficiencies and tunings of planar $200 \mu \mathrm{m}$ thick SE sensors after irradiation. The irradiation levels of the measured devices are: SCC60, PS2 and SCC61 $\Rightarrow 5 \cdot 10^{15} \frac{\mathrm{n}_{\mathrm{eq}}}{\mathrm{cm}^{2}}$ and the neutron irradiated LUB4 $\Rightarrow 4 \cdot 10^{15} \frac{\mathrm{n}_{\mathrm{eq}}}{\mathrm{cm}^{2}}$. 

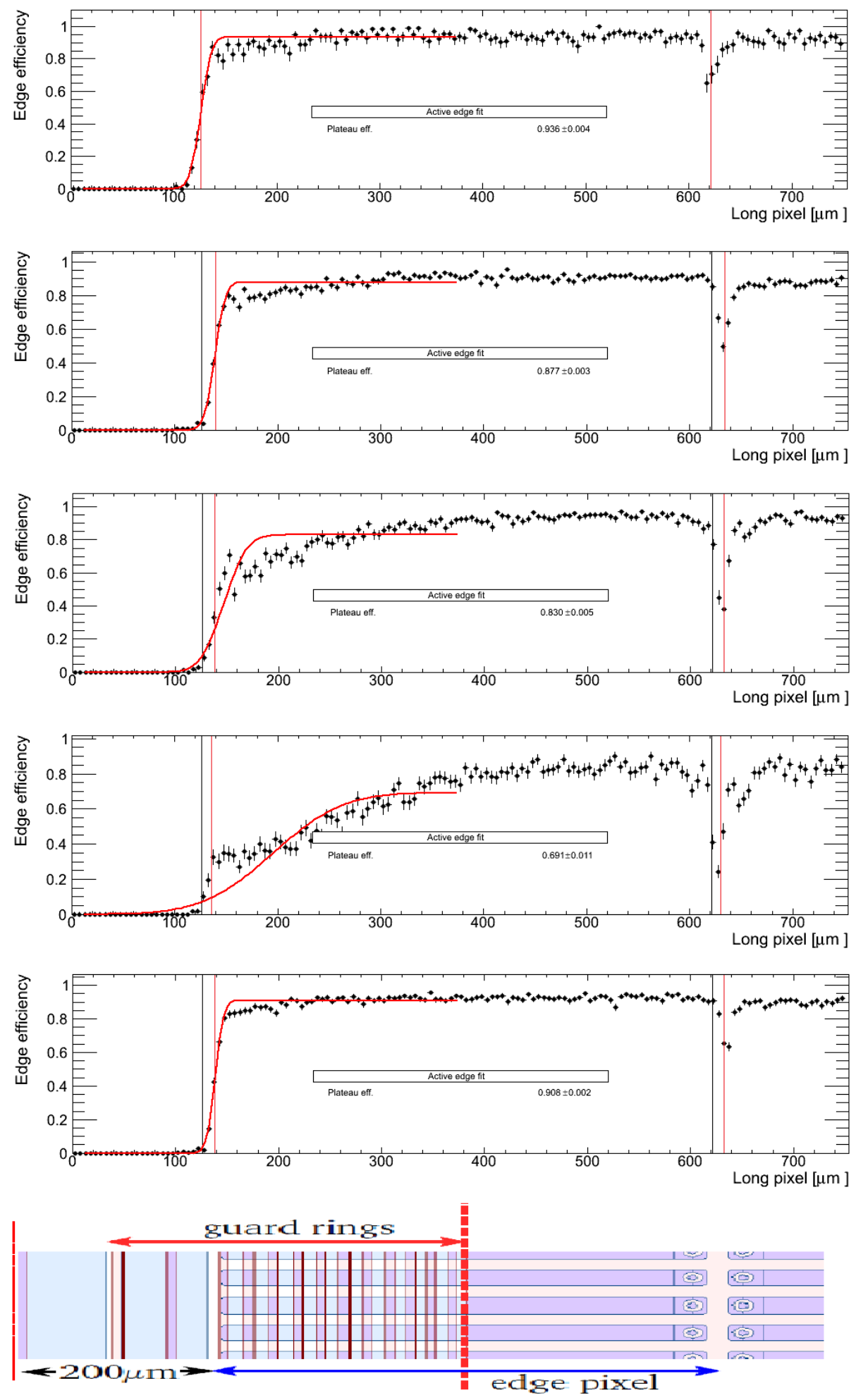

Figure 7.9: Edge efficiency of LUB2 device for bias voltages of $1000 \mathrm{~V}, 800 \mathrm{~V}, 600 \mathrm{~V}, 400 \mathrm{~V}$ and finally ramped to $1000 \mathrm{~V}$ again. 


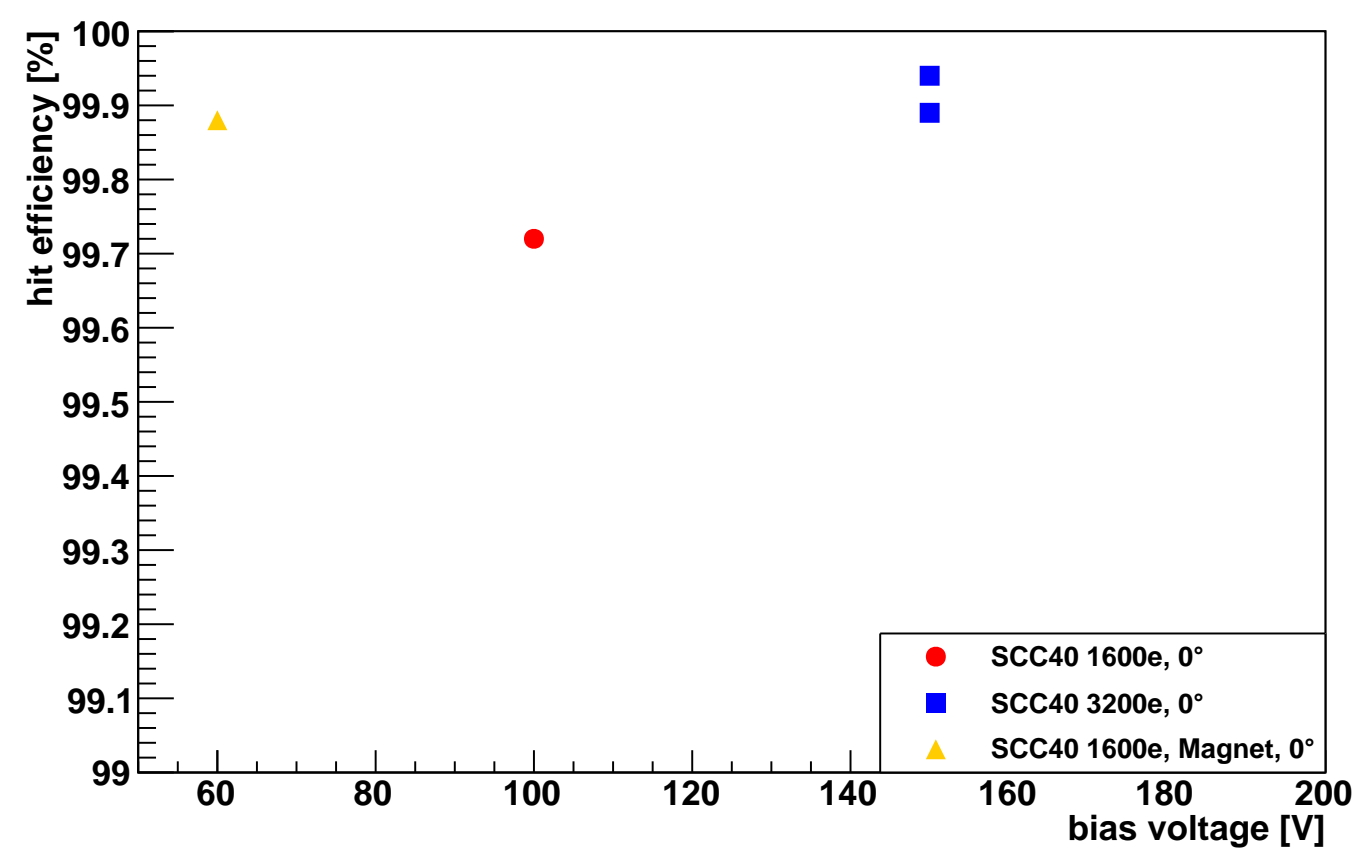

Figure 7.10: Average hit efficienciy of $200 \mu \mathrm{m}$ thick planar slim edge sensors, measured for different voltages, thresholds and in magnetic field. The efficiency fulfils the IBL requirements under all tested conditions.
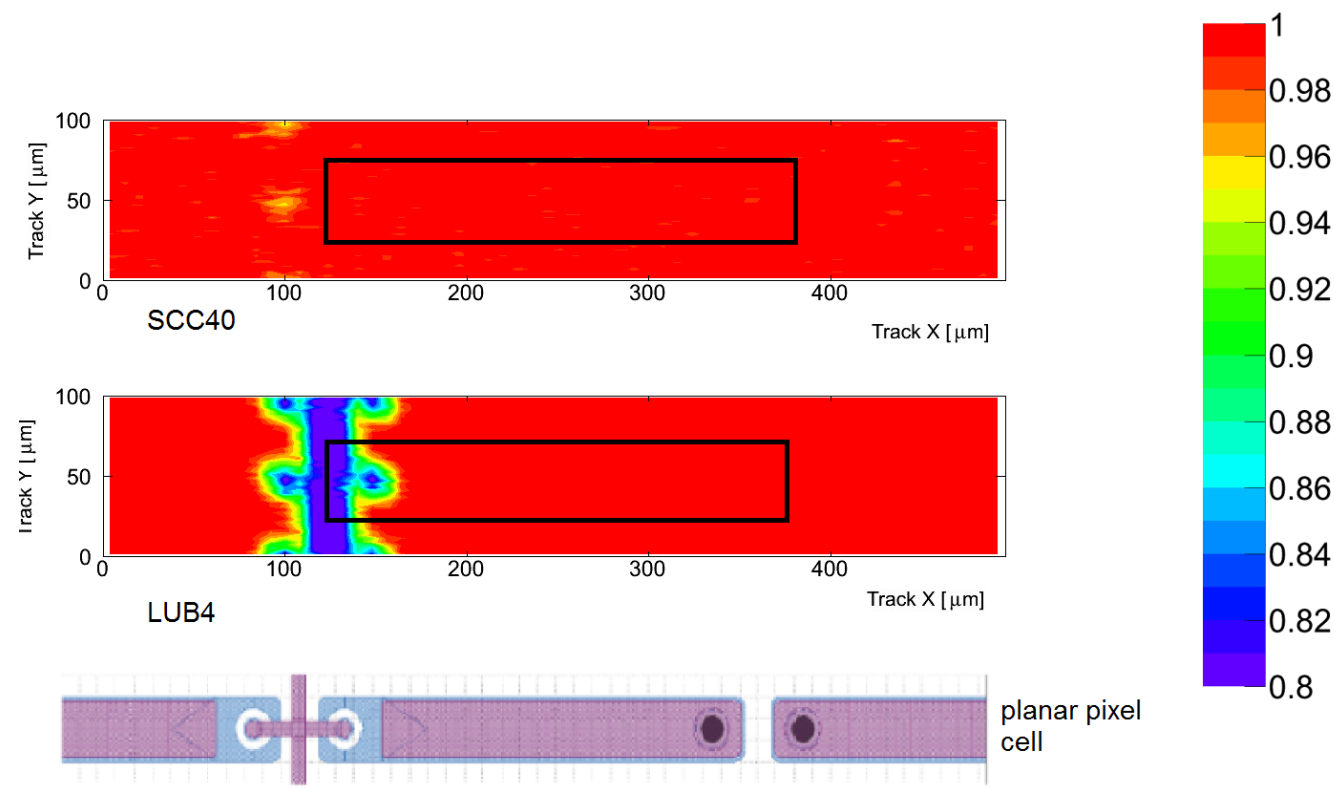

0.88

0.86

0.84

0.82

0.8

Figure 7.11: In-pixel efficiency for devices SCC40 (planar, slim edge, unirradiated) and LUB4 (planar, slim edge, irradiated) at perpendicular incidence, folded into one pixel and half of its neighbours. For comparison, the pixel cell structure is printed below. The drop in efficiency for irradiated planar devices is strongest at the bias rail and bias dot structure. 


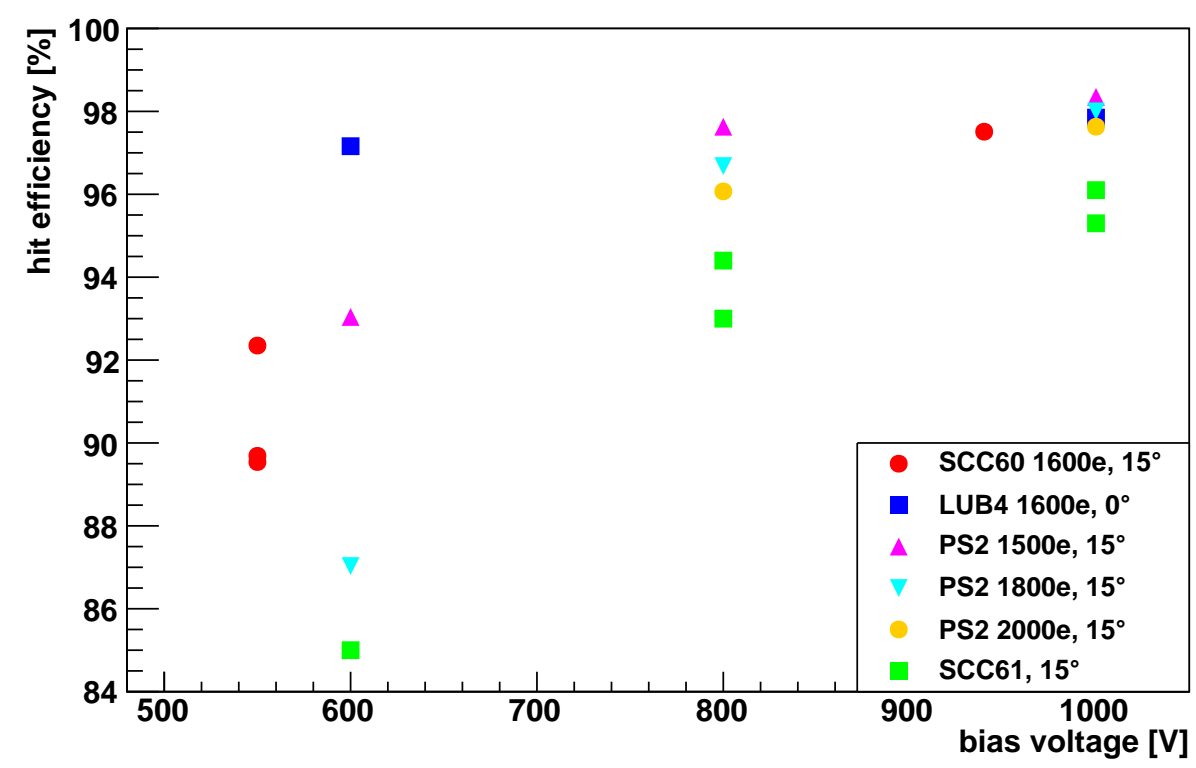

Figure 7.12: Average hit efficiencies of $200 \mu \mathrm{m}$ thick planar slim edge sensors after irradiation, measured for different voltages and thresholds.

bias voltage.

When the bias voltage is decreased to values below the depletion voltage, the noise level of the sensor increases, which has a negative impact on the hit efficiency. An indication for the increasing noise is shown in figure 7.14. The noisefloor for the depletion voltage of $1000 \mathrm{~V}$ is at around $6 \%$ of the peak height, while for $600 \mathrm{~V}$ bias voltage the noise floor is at $15 \%$ compared to the peak height. The monitoring of leakage currents was not reliable and frequent enough for the irradiated thin SE devices to draw clear conclusions for a dependency between the currents and the hit efficiency.

As shown, the irradiated slim edge devices fulfil the $97 \%$ average efficiency IBL requirement at sufficient bias voltages. The efficiency of device SCC61 is lower, but it should be noted that a large fraction of pixels had to be masked for this device. Since the analysis processors automatically mask all neighbouring pixels of a masked pixel, the efficiency map shown in figure 7.15 (right) is not filled for a large fraction of the sensor. Thus, the calculated value should be taken with a grain of salt. In summary, the slim edge design showed sufficient efficiency throughout the whole length of the edge pixels, even after irradiation. Furthermore, the average efficiency limit of $97 \%$ after irradiation was exceeded for these devices, within the limits of maximum $1000 \mathrm{~V}$ bias voltage. Due to the higher geometric efficiency and lower material budget, compared to the other planar designs, IBL management decided to build the central part of the IBL detector, using $200 \mu \mathrm{m}$ thick slim edge sensors. 

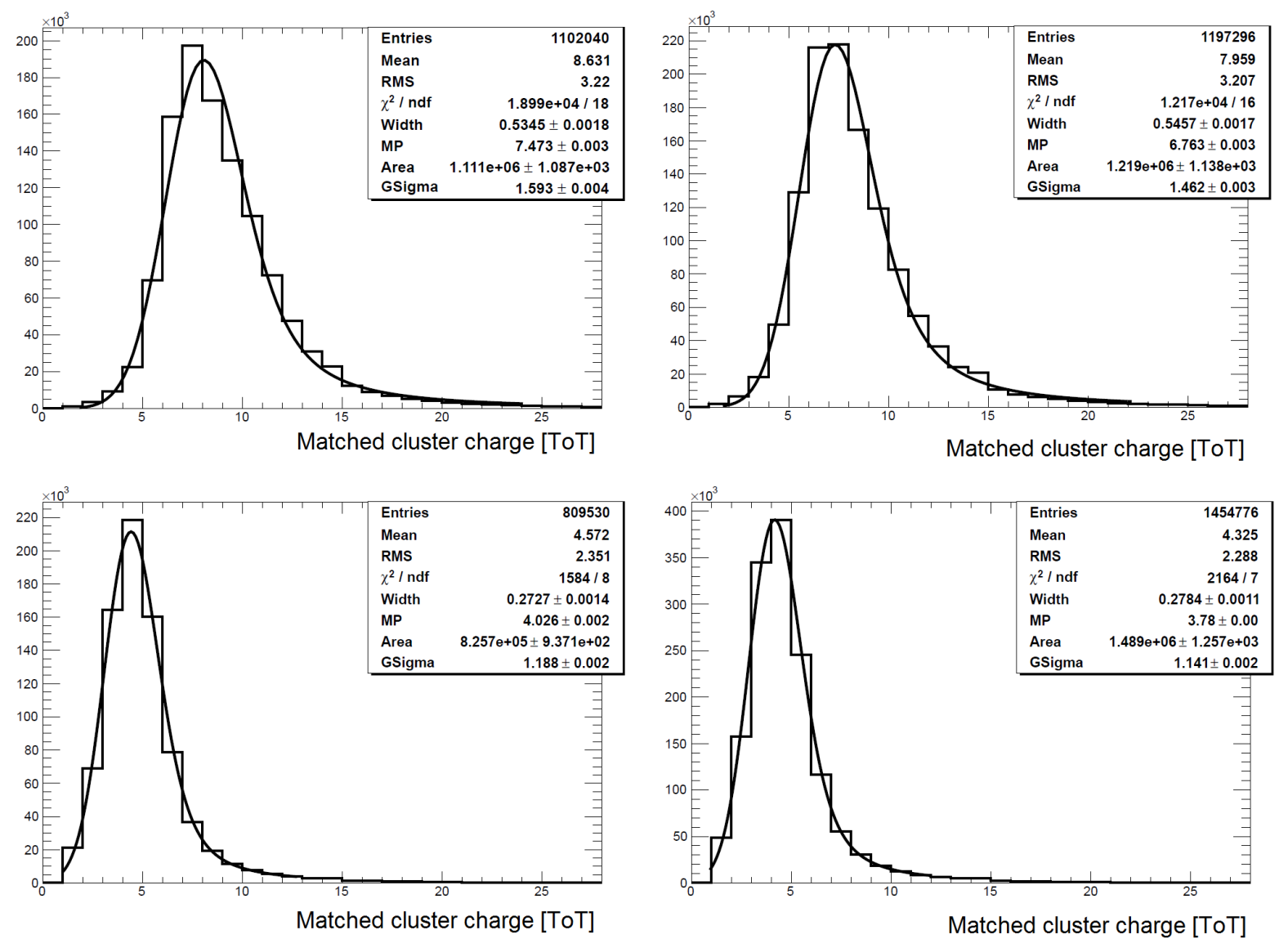

Figure 7.13: Histogram a) shows the detected cluster charge for device PS2 at a bias voltage of $1000 \mathrm{~V}$ and a threshold of $1500 \mathrm{e}^{-}$in units of ToT. In histogram b) the same measurement again at $1000 \mathrm{~V}$ bias voltage is shown, but for a signal threshold of $1800 \mathrm{e}^{-}$. Histogram c) shows the cluster ToT measurement again for device PS2 at a bias voltage of $600 \mathrm{~V}$ and at a threshold of $1800 \mathrm{e}^{-}$. In histogram d) the measurement was repeated for a bias voltage of $600 \mathrm{~V}$ and again a threshold of $1800 \mathrm{e}^{-}$.
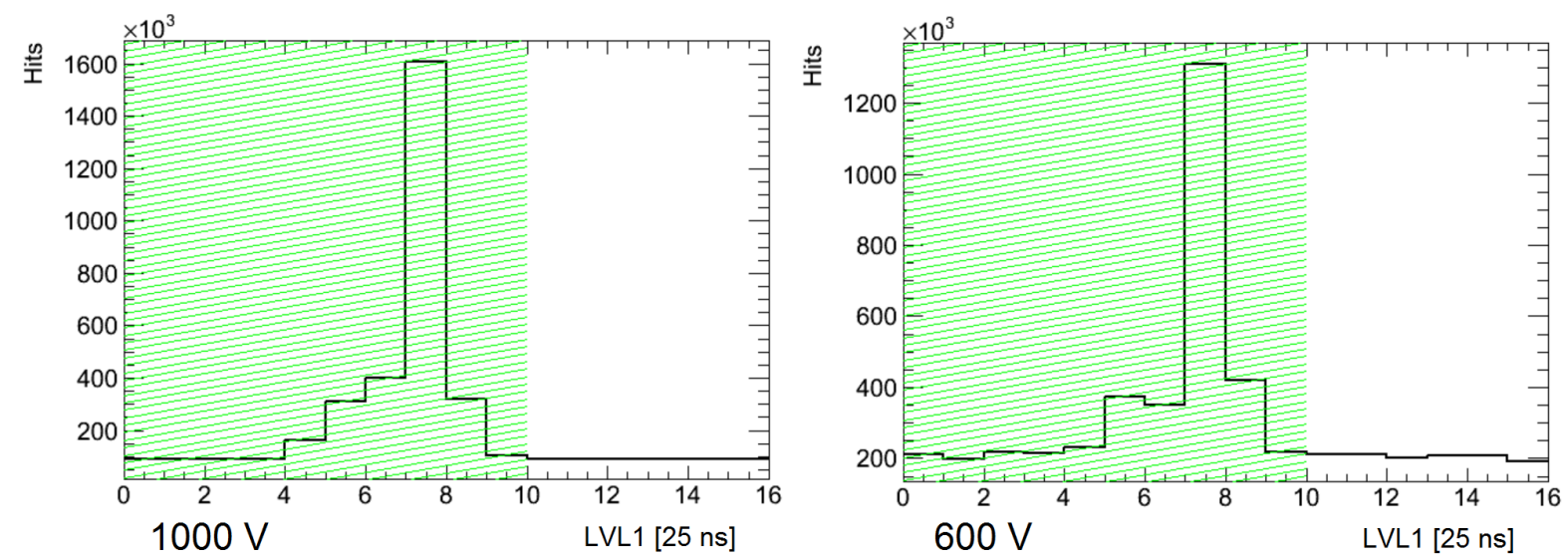

Figure 7.14: Comparison of the LVL1 distribution for device PS2 at $1000 \mathrm{~V}$ on the left and $600 \mathrm{~V}$ on the right. For the higher bias voltage the noise floor is at $6 \%$ of the peak height, for the lower bias voltage the noise floor is at $15 \%$ of the peak height. The green area shows the default acceptance window of LVL1 values between $[0,10]$. 

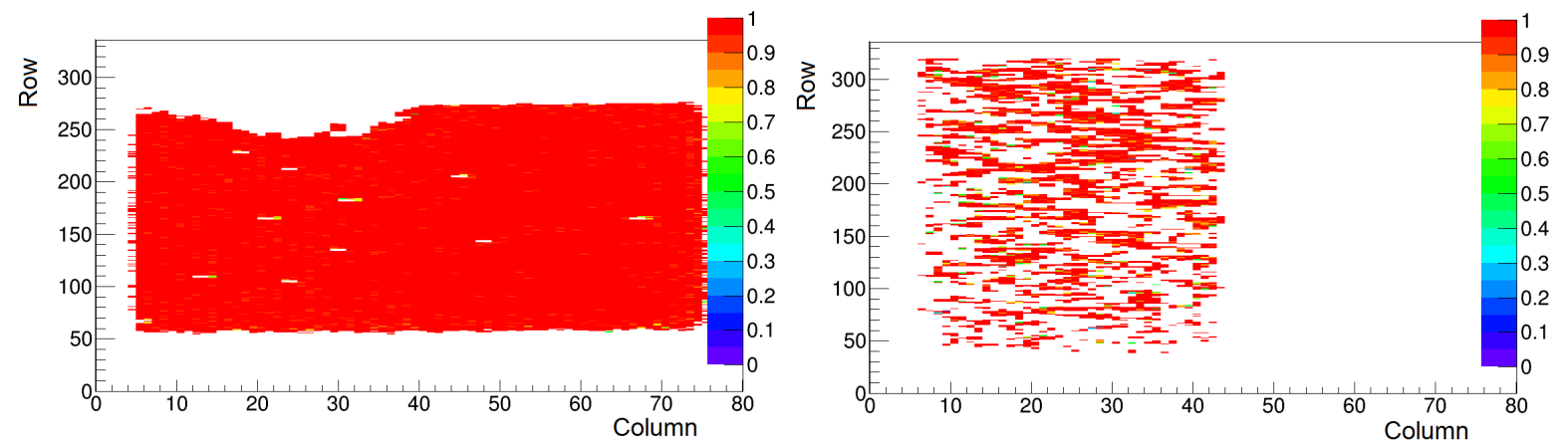

Figure 7.15: Comparison of the efficiency maps for device PS2 (left) and SCC61 (right). Since a significant fraction of pixels had to be masked for SCC61 and the analysis automatically masks the neighbour pixels of each masked pixel, the efficiency calculation of this device is not representative. Note: the missing region in the efficiency map of PS2 is caused by the reference device FBK13, which had a bump-bonding issue in this region. Since FBK13 was the reference device, no tracks could be matched in that region. 


\subsection{D Pixel Sensors - Results}

The 3D R\&D group proposed to investigate double sided sensors and full 3D devices as new technology and potential sensor candidates for the IBL detector. As described in section 4.2.1, one of the main differences between the two designs is the length of the electrodes. For double sided sensors, the electrodes do not reach through the whole sensor thickness, while in full 3D devices the electrodes extend from the backside to the frontside of the sensor. Since 3D silicon sensors were never used before in ATLAS, it had to be investigated whether their performance is comparable to planar sensors. In the following, the results of both types of sensors before and after irradiation are presented.

\subsubsection{Double Sided Designs}

Like for planar devices, the average hit efficiency was of highest interest during the measurements. Figure 7.16 shows the average hit efficiency for the unirradiated device CNM55. Since the documented leakage currents of the irradiated device CNM55 was below $0.3 \mu \mathrm{A}$ for the listed measurements, the values are not listed in detail, because their effect is negligible. The device was tuned to a signal of $8 \mathrm{ToT}$ at $20 \mathrm{ke}^{-}$.

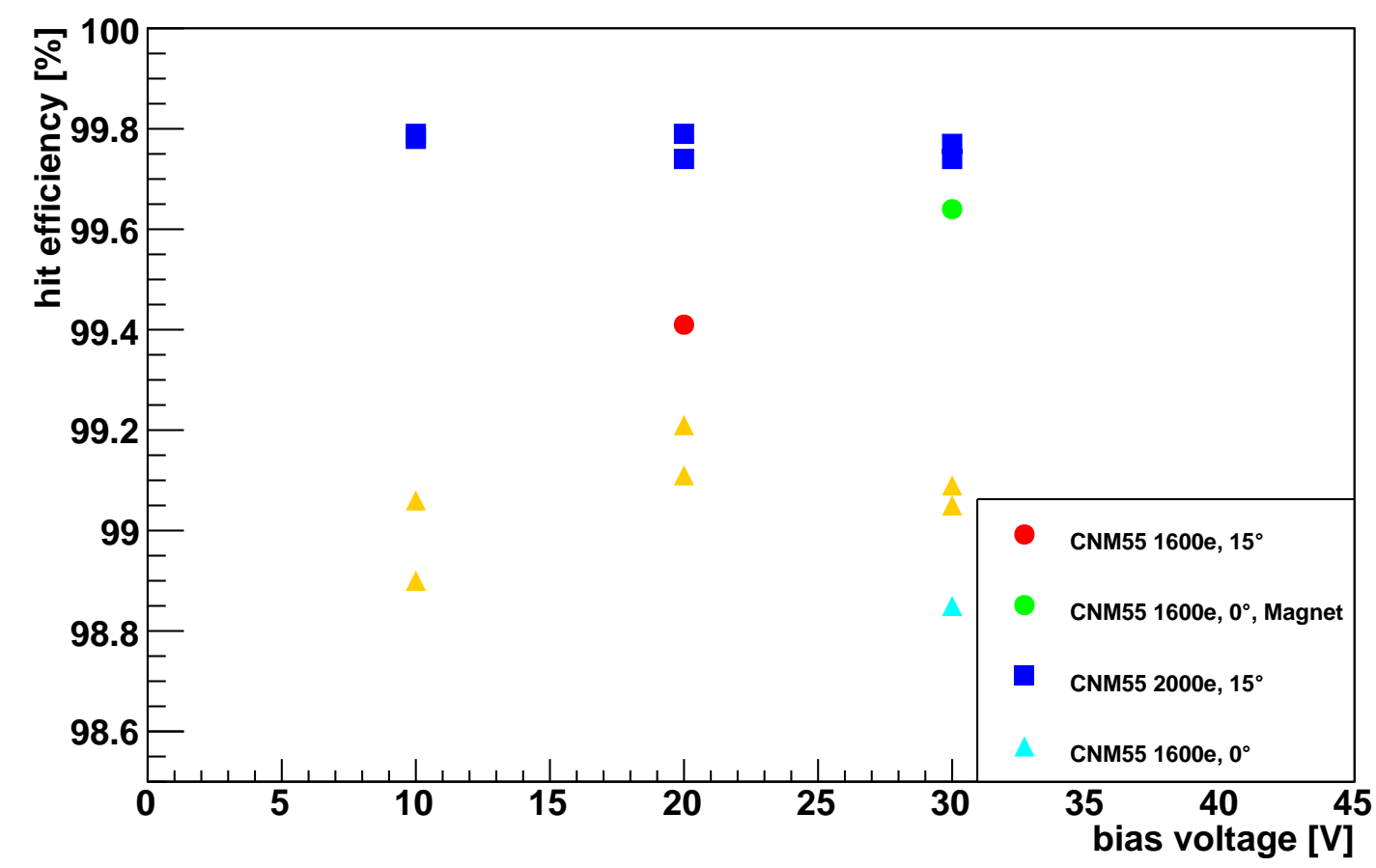

Figure 7.16: Average hit efficiencies of unirradiated double sided 3D devices for different threshold settings, bias voltages and incident angles.

The measurement of CNM55 at $30 \mathrm{~V}$ bias voltage and perpendicular incidence shows the lowest efficiency in this series, at a value of $98.8 \%$. The same device under the same measurement conditions, except that a magnetic field was applied, already shows an increase in hit efficiency to $99.6 \%$. Due to the helix shape of the particle tracks in strong magnetic fields, the particles do not only cross the electrode anymore, but are detected in the sensitive area of the sensor. 

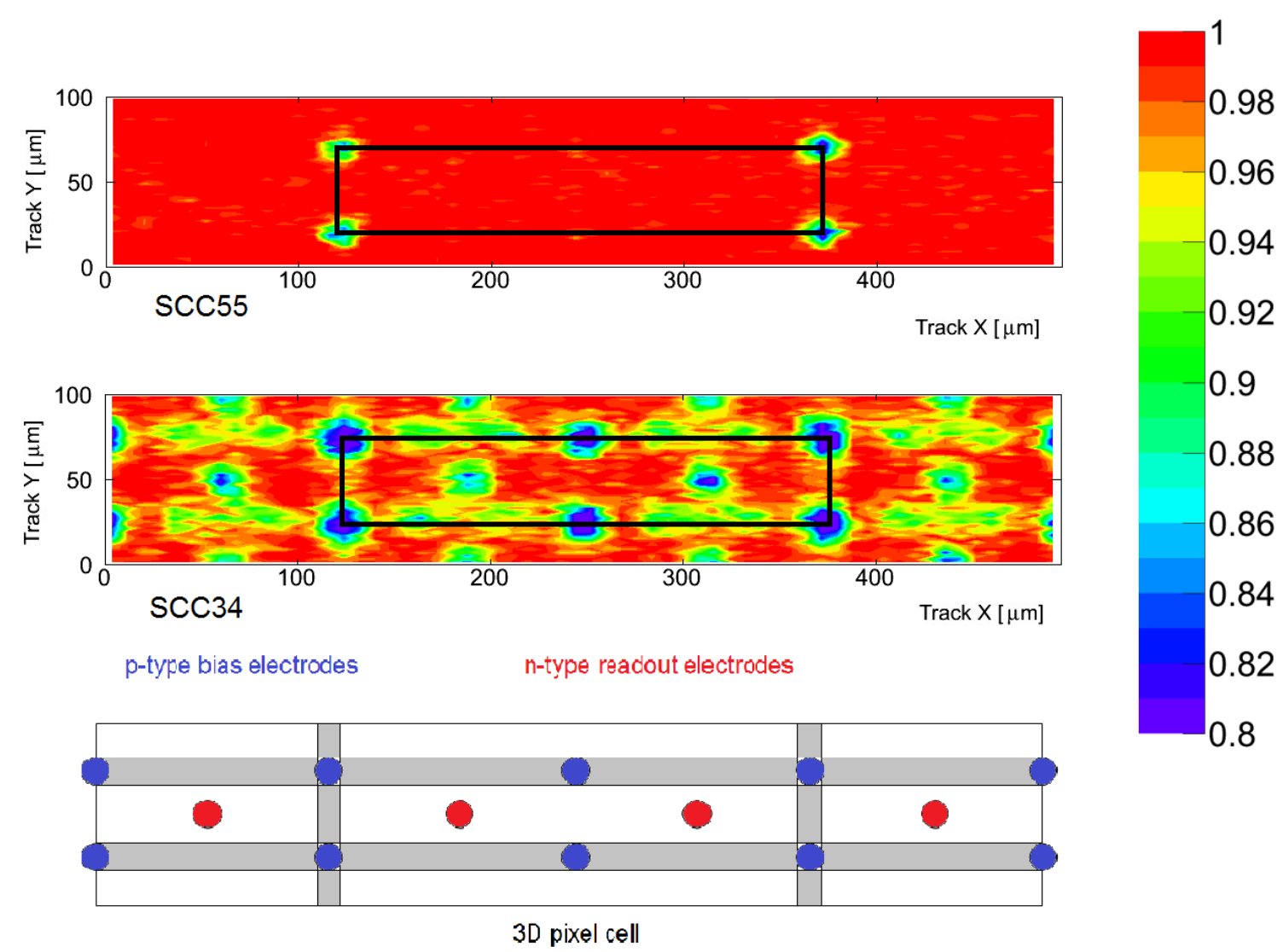

Figure 7.17: In-pixel efficiency for devices SCC55 (3D, double sided, unirradiated) and SCC34 (3D, double sided, unirradiated) at perpendicular incidence, folded into one pixel and half of its neighbours. For comparison, the pixel cell structure is printed below.

In figure 7.17, the in-pixel efficiency for SCC55, an unirradiated double sided 3D sensor, and in comparison for SCC34, which is a double sided 3D device, irradiated up to the specified IBL fluence of $5 \cdot 10^{15} \frac{\mathrm{n}_{\mathrm{eq}}}{\mathrm{cm}^{2}}$, is shown. SCC55 only has an efficiency drop in the region of the p-type columns. For SCC34 an efficiency drop in the columns and along the pixel cell edges can be seen for the used bias voltage, which is lower than the depletion voltage. This leads to a lower average efficiency at this setting. The efficiency loss inside the electrodes is subject of further investigations to fully understand the effects that cause it.

Several double sided 3D sensors were irradiated for measurements in IBL testbeams. Compared to planar devices, a lower depletion voltage and lower leakage currents were expected for 3D devices, due to their design. Figure 7.18 shows the average hit efficiencies for the tested devices, the measurement conditions are listed in table 7.5.

As this overview shows, with the exception of SCC82, the irradiated double sided 3D sensors show a high average hit efficiency at comparably low bias voltages of $140 \mathrm{~V}$. During the measurements it turned out that the applied bias voltages between $140 \mathrm{~V}$ and $170 \mathrm{~V}$ were not sufficient for full depletion. A very interesting effect can be seen for the impact of the leakage current on the average hit efficiency. For all tested irradiated double sided 3D devices an increase of the bias voltage had a positive effect on the average hit efficiency, as long as the leakage current did not rise. The only exception is noted 


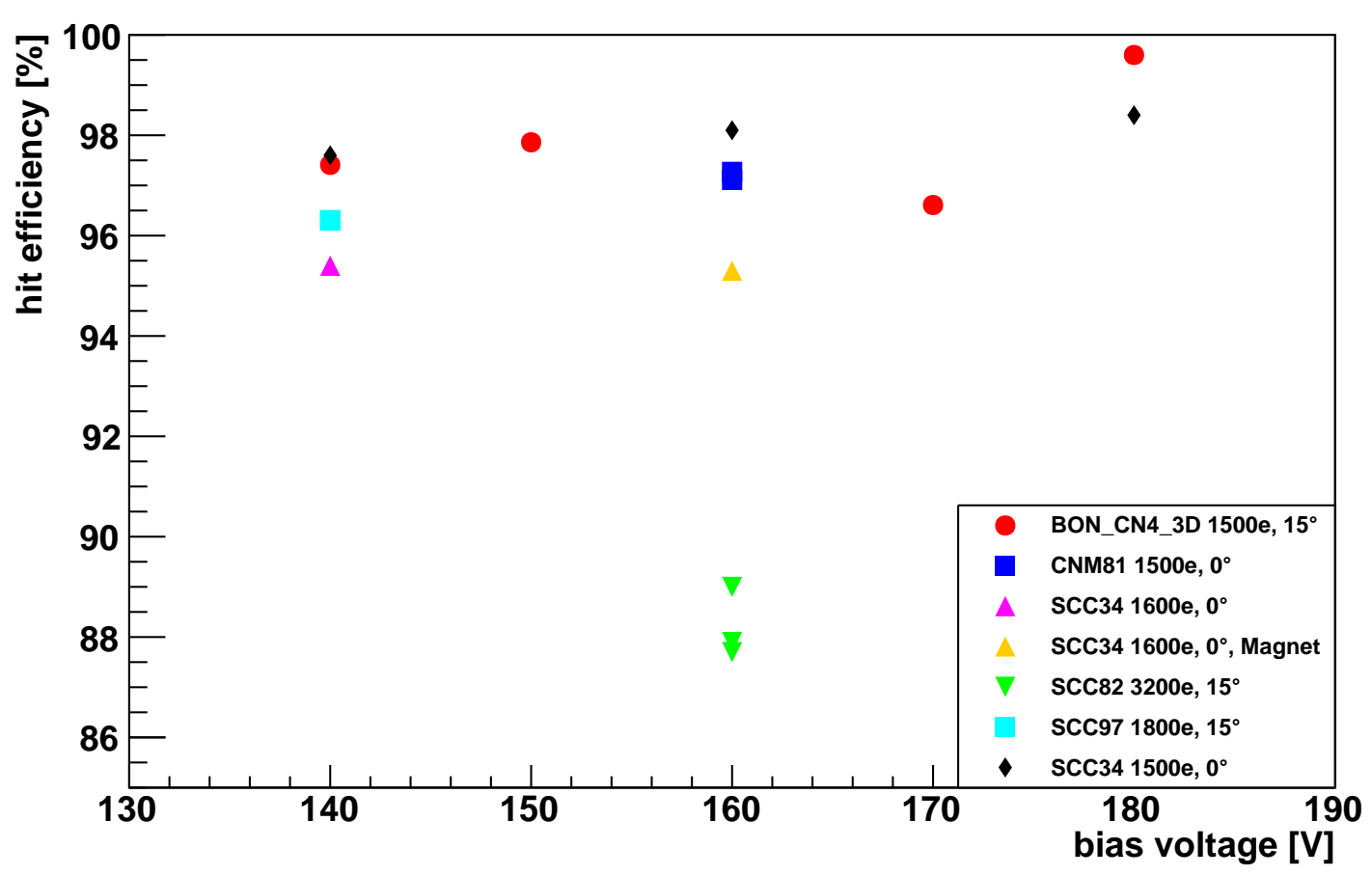

Figure 7.18: Average hit efficiencies of irradiated double sided 3D devices. The large drop in efficiency even at non-perpendicular incidence of device SCC82 compared to the other devices indicates, that the collected charge of the device is around the threshold value of $3 \mathrm{ke}^{-}$.

for device CNM81 at a bias voltage of $180 \mathrm{~V}$, where the efficiency reached its peak value, although the leakage current increased. This can be taken as an indication that only at the bias voltage of $180 \mathrm{~V}$ the device was fully depleted. This can also be confirmed by the edge efficiency analysis of devices SCC34 and SCC97 at $140 \mathrm{~V}$ bias voltage, shown in figure 7.19, where the edge pixels show a reduced hit efficiency. The 3D edge sensor design includes a distance of $200 \mu \mathrm{m}$ between the edge pixels and the cutting edge. In the edge efficiency analysis, the 50\% efficiency point for SCC 34 is at the end of the edge pixels, which means an inactive edge width of $\approx 200 \mu \mathrm{m}$. For SCC97 the statistics was too low to achieve a reliable result.

The low hit efficiency of device SCC 82 can be explained by the high applied signal threshold of $3200 \mathrm{e}^{-}$ for this device. Combined with the tuning to a signal of 8 ToT at $20 \mathrm{ke}^{-}$, part of the signal was cut, since the expected signal charge is small in irradiated sensors, which leads to low ToT values at the chosen tuning. This effect can be seen in figure 7.20. The resulting distribution is clearly shifted to small values, where the combination of the applied signal threshold and the tuning leads to a suppression of small detected charges. Since other measurements indicated that the depletion voltage for irradiated devices is around $180 \mathrm{~V}$, the low hit efficiency is a result of the combination of all these effects.

In summary, double sided 3D sensors proved that they fulfil the required IBL specifications. The required depletion voltage after IBL fluences seems to be around $180 \mathrm{~V}$. Very interesting is the clear dependency between leakage current and hit efficiency. 


\begin{tabular}{|l|c|c|c|c|c|}
\hline sensor ID & bias voltage [V] & $\mathrm{I}_{\text {leak }}[\mu \mathrm{A}]$ & average eff. [\%] & tuning [ToT@ $\left.\mathrm{ke}^{-}\right]$ & threshold $\left[\mathrm{e}^{-}\right]$ \\
\hline \hline CNM81 & 140 & 45 & 97.41 & $8 @ 20$ & 1500 \\
\hline & 150 & 35 & 97.86 & $8 @ 20$ & 1500 \\
\hline & 170 & 50 & 96.61 & $8 @ 20$ & 1500 \\
\hline & 180 & 78 & 99.60 & $8 @ 20$ & 1500 \\
\hline SCC34 & 140 & 150 & 95.40 & $8 @ 20$ & 1600 \\
\hline & 160 & 220 & 95.30 & $8 @ 20$ & 1600 \\
\hline SCC82 & 160 & 72 & 87.90 & $8 @ 20$ & 3200 \\
\hline & 160 & 76 & 87.69 & $8 @ 20$ & 3200 \\
\hline & 160 & 26 & 89.00 & $8 @ 20$ & 3200 \\
\hline SCC97 & 140 & 32 & 96.30 & $8 @ 20$ & 1800 \\
\hline & 140 & 31 & 96.31 & $8 @ 20$ & 1800 \\
\hline
\end{tabular}

Table 7.5: Bias voltages, leakage currents, average efficiencies and tunings of irradiated double sided 3D devices. The irradiation level of the measured devices is $5 \cdot 10^{15} \frac{\mathrm{n}_{\mathrm{eq}}}{\mathrm{cm}^{2}}$.

\subsubsection{Full 3D Sensors}

To investigate and compare the performance of full 3D sensors to double sided 3D devices, a set of full 3D sensors were investigated. Naturally, the same qualification criteria as for all other designs had to be matched. Like for double sided 3D sensors, the full 3D devices first had to deliver a proof of concept and show whether their performance is comparable to planar sensors or not. In section 4.2.1 the concept of full 3D sensors was already introduced. The basic difference compared to double sided devices is that the doped electrodes reach through the whole sensor thickness. For the full 3D device FBK13, figure 7.21, a drop in efficiency at perpendicular incidence in both, the n-doped and the p-doped, electrode types can be seen already before irradiation. Device FBK13 is an unirradiated full 3D device, which has been used in several testbeam campaigns. Therefore, efficiency measurements could be performed under different conditions, as shown in figure 7.22. The device shows a very high average hit efficiency of at least $98.5 \%$ under all tested conditions. All noted leakage currents were below $0.1 \mu \mathrm{A}$, thus the list of leakage currents is skipped for this device. For all measurements, FBK13 was tuned to a charge response of 8 ToT at $20 \mathrm{ke}^{-}$. Notable is the fact that at a threshold setting of $1600 \mathrm{e}^{-}$in total nine different run blocks of in total almost 240 runs were evaluated for the efficiency measurement, containing in total almost 23.1 million events. The resulting value only varied between $99.96 \%$ and $99.97 \%$ hit efficiency, which gives the impression of just one data point in the histogram.

A set of full 3D devices were irradiated to different fluences. The irradiation levels for the presented devices are $2 \cdot 10^{15} \frac{\mathrm{n}_{\mathrm{eq}}}{\mathrm{cm}^{2}}$ for SCC $90,6 \cdot 10^{15} \frac{\mathrm{n}_{\mathrm{eq}}}{\mathrm{cm}^{2}}$ for FBK11 and $\geq 5 \cdot 10^{15} \frac{\mathrm{n}_{\mathrm{eq}}}{\mathrm{cm}^{2}}$ for SCC $87^{5}$. Thus, FBK11 is the most interesting device in terms of performance for irradiation fluences even higher than required by IBL. The calculated average efficiencies under different conditions are shown in figure 7.23, the detailed measurement conditions are listed in table 7.6. It should be noted, that due to the high irradiation

\footnotetext{
${ }^{5}$ The irradiation facility had monitoring problems and could only ensure a minimal measured fluence for the device
} 


\begin{tabular}{|l|c|c|c|c|c|}
\hline sensor ID & bias voltage [V] & $\mathrm{I}_{\text {leak }}[\mu \mathrm{A}]$ & average eff. [\%] & tuning [ToT@ $\left.\mathrm{ke}^{-}\right]$ & threshold $\left.\mathrm{e}^{-}\right]$ \\
\hline \hline FBK11 & 100 & 60 & 95.97 & $8 @ 20$ & 1500 \\
\hline & 110 & 60 & 96.00 & $8 @ 20$ & 1500 \\
\hline & 120 & 33 & 96.28 & $8 @ 20$ & 1500 \\
\hline & 130 & 114 & 96.32 & $8 @ 20$ & 1500 \\
\hline & 140 & 114 & 96.34 & $8 @ 20$ & 1500 \\
\hline & 150 & 146 & 96.58 & $8 @ 20$ & 1500 \\
\hline & 160 & 114 & 96.40 & $8 @ 20$ & 1500 \\
\hline & 160 & 46 & 83.70 & $8 @ 20$ & 1600 \\
\hline & 140 & 41 & 97.76 & $8 @ 20$ & 1800 \\
\hline & 150 & 146 & 95.11 & $8 @ 20$ & 1800 \\
\hline \multirow{3}{*}{ SCC87 } & 160 & 234 & 93.83 & $8 @ 20$ & 1800 \\
\hline & 140 & 32 & 93.10 & $8 @ 20$ & 3200 \\
\hline SCC90 & 140 & 30 & 93.40 & $8 @ 20$ & 3200 \\
\hline & 160 & 33 & 99.21 & $8 @ 20$ & 3200 \\
\hline
\end{tabular}

Table 7.6: Bias voltages, leakage currents, average efficiencies and tunings of irradiated full 3D devices. Note: the measurement of FBK11 at the threshold of $1600 \mathrm{e}^{-}$was done at perpendicular incidence, while all other measurements were done for $15^{\circ}$ rotation along the $\phi$-direction. 

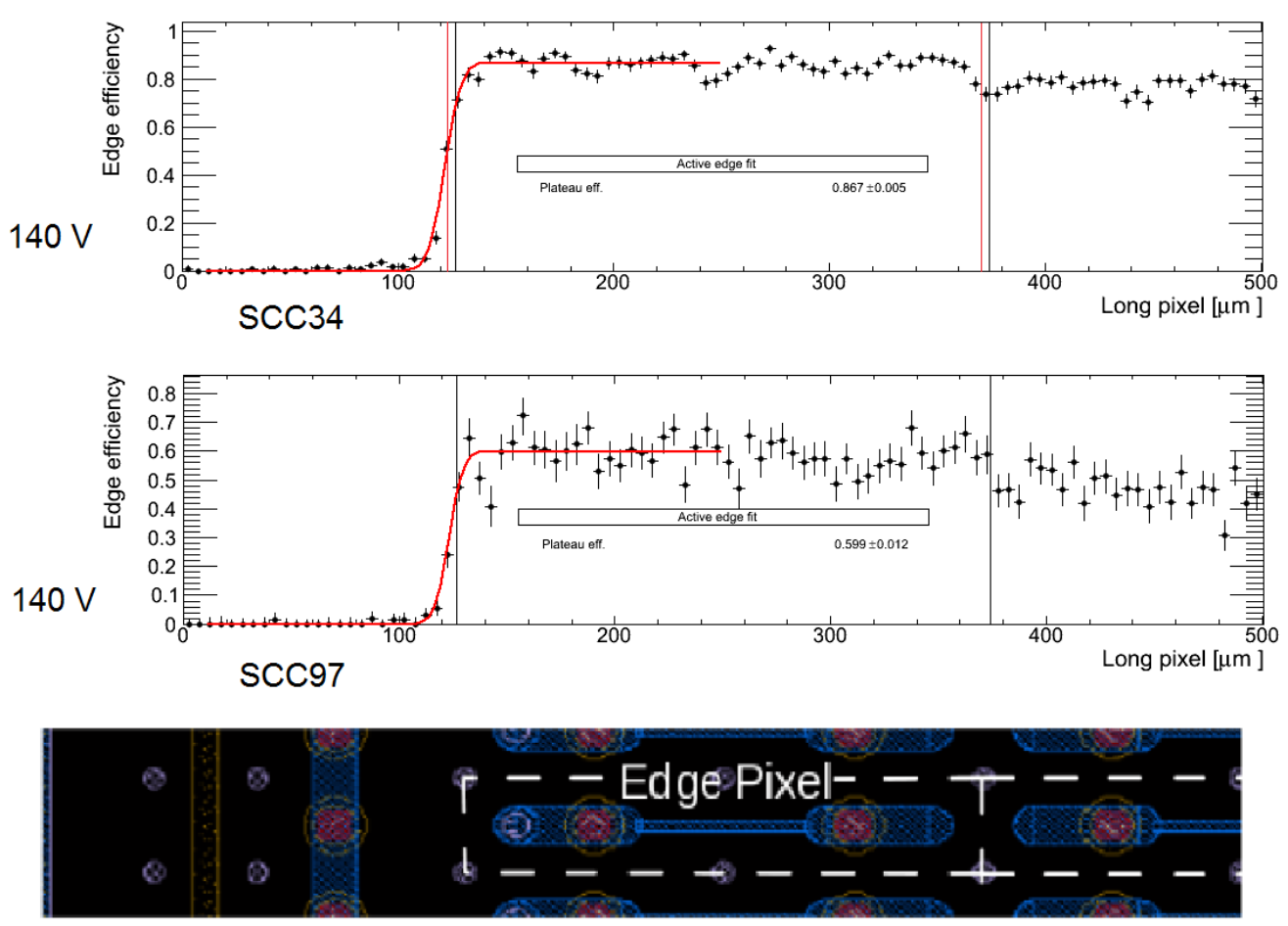

Figure 7.19: Edge efficiencies of devices SCC34 and SCC97 at a bias voltage of $140 \mathrm{~V}$. The plateau efficiency values and the visible structures in the efficiency distribution indicate, that the devices were not fully depleted. Even in underdepleted operation the edge efficiency level is uniform over the whole edge pixel width.

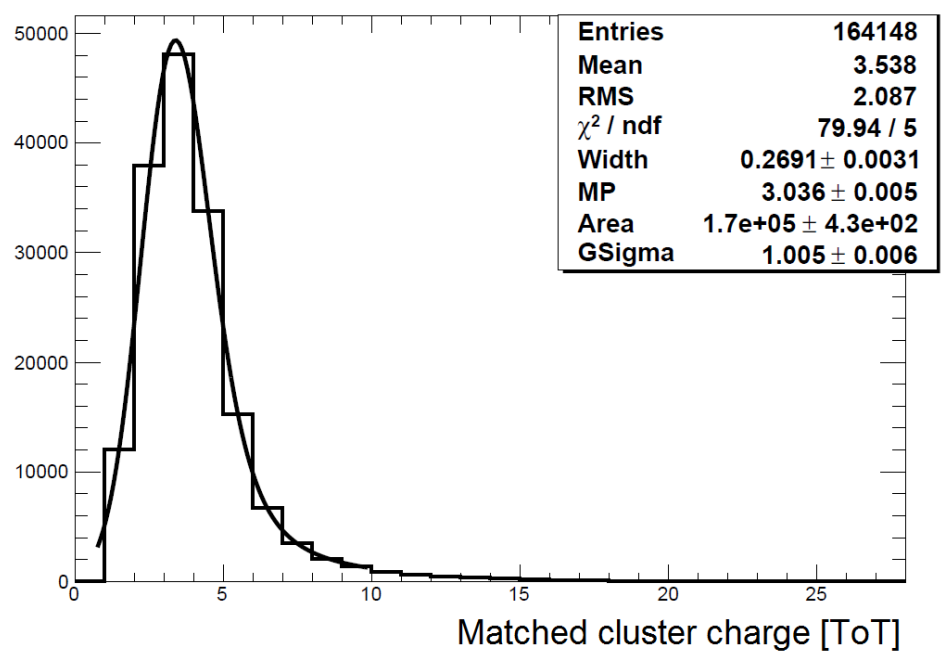

Figure 7.20: ToT distribution of device SCC82 at a bias voltage of $160 \mathrm{~V}$, signal threshold of $3200 \mathrm{e}^{-}$and a feedback tuning to $8 \mathrm{ToT}$ at $20 \mathrm{ke}^{-}$. The distribution is shifted to low values, where very small charges are cut by the applied signal threshold.

level of FBK11, a large fraction of pixels had to be masked for the measurements. The data point at a threshold of $1600 \mathrm{e}^{-}$at perpendicular incidence was taken during a later testbeam campaign than the 


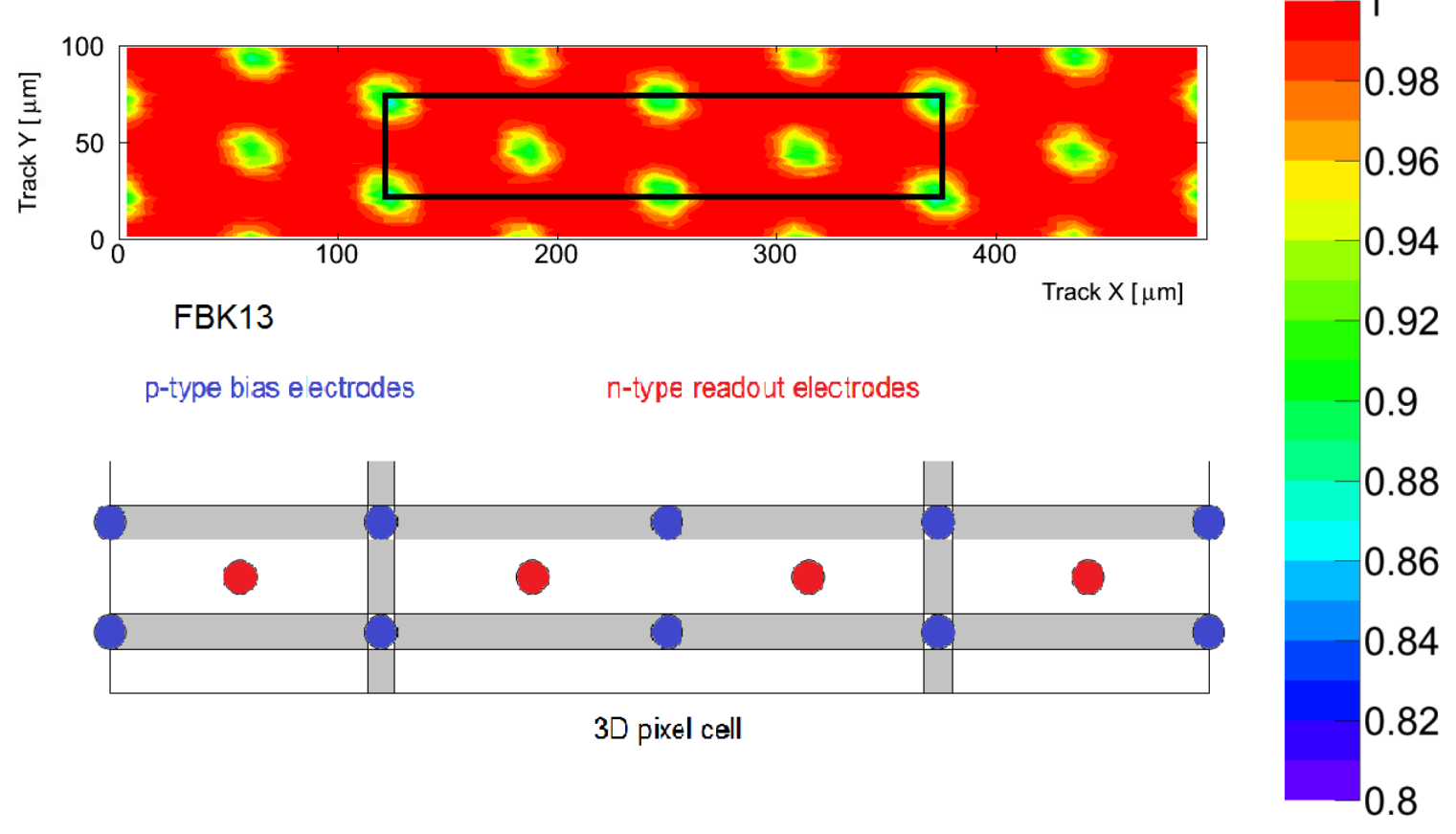

Figure 7.21: In-pixel efficiency for device FBK13 (full 3D, unirradiated) at perpendicular incidence, folded into one pixel and half of its neighbours. The 3D pixel cell structure is drawn below.

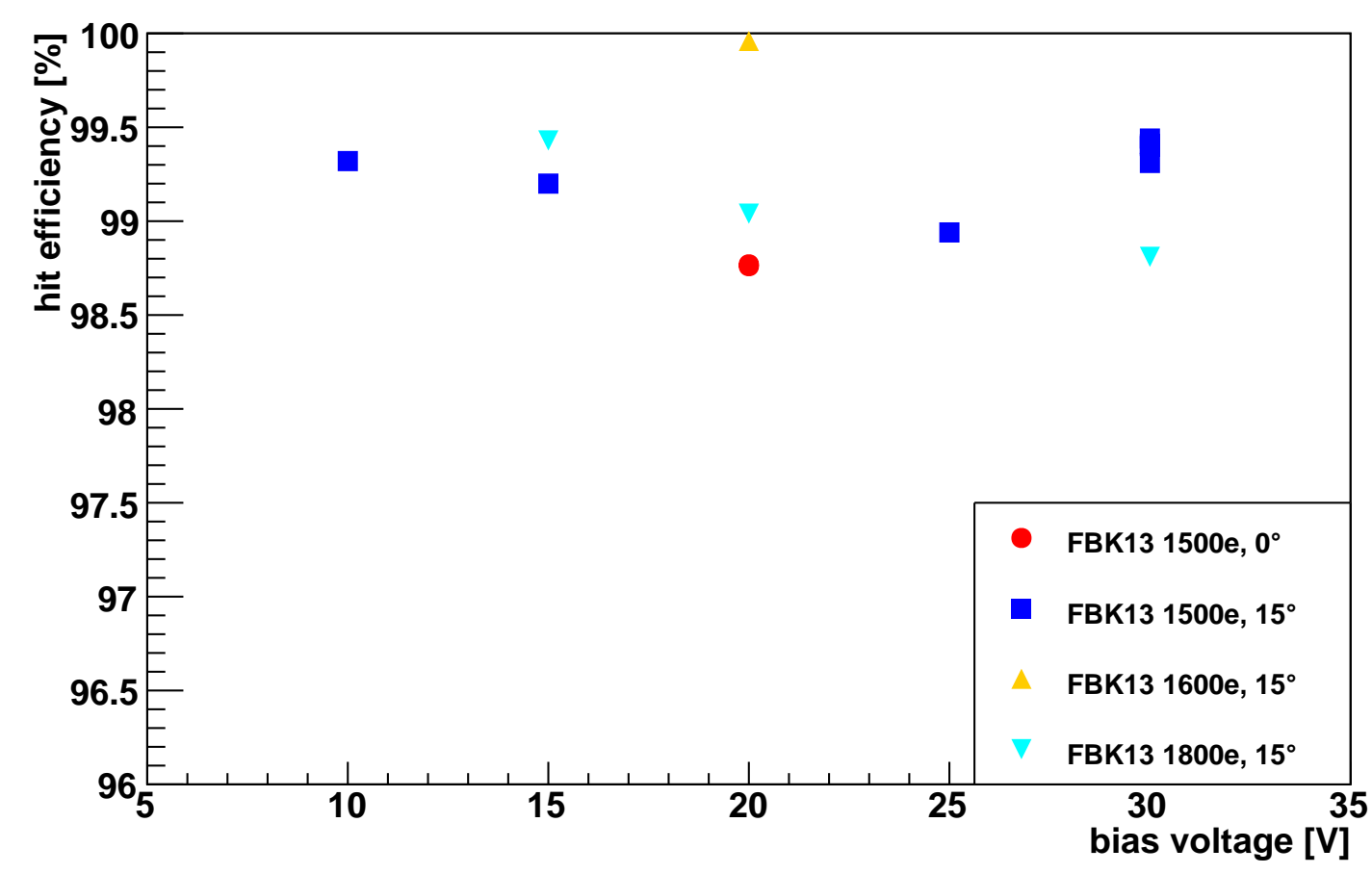

Figure 7.22: Average hit efficiencies of unirradiated full 3D devices at different signal thresholds and bias voltages. 


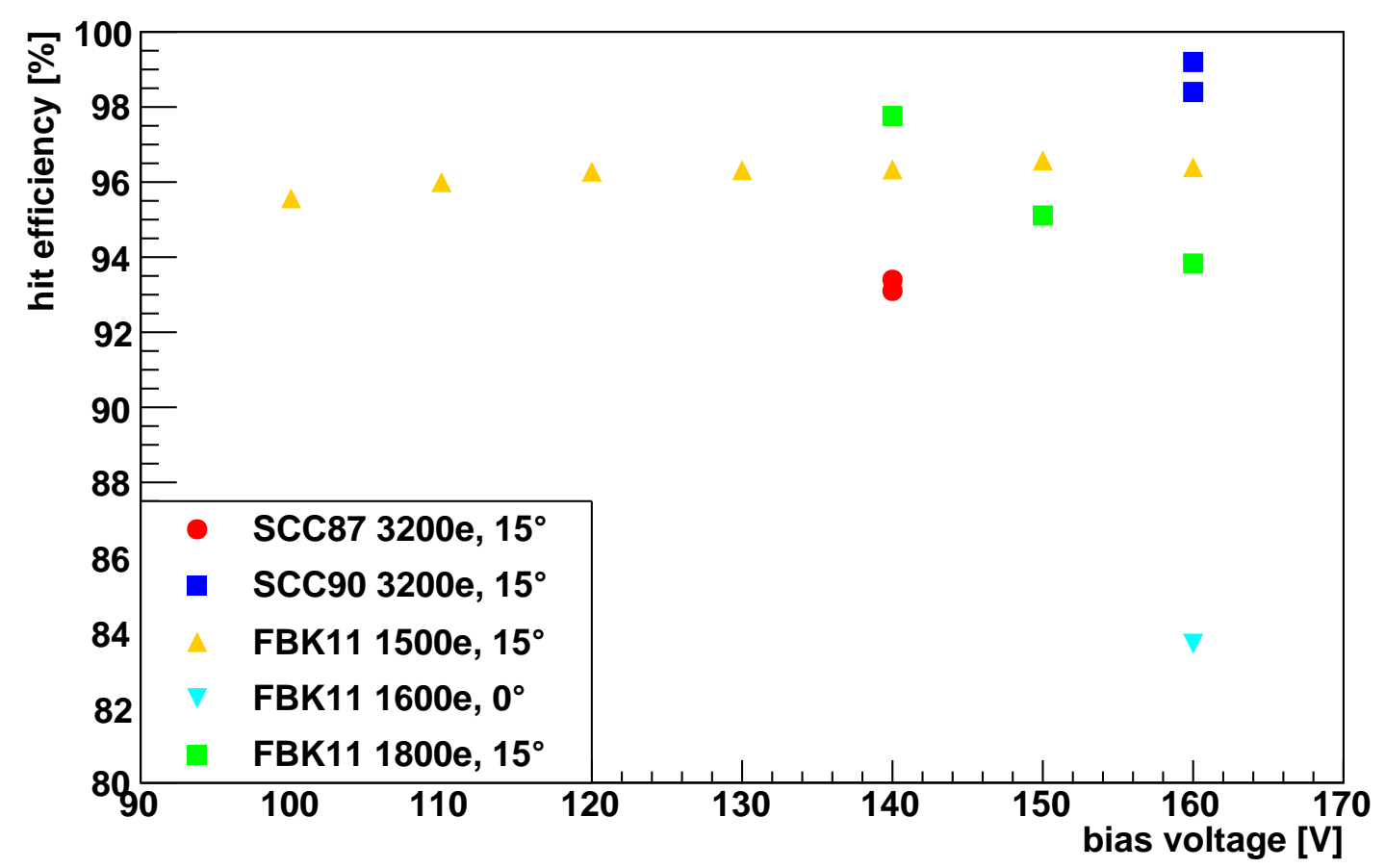

Figure 7.23: Average hit efficiencies of irradiated full 3D devices for a set of voltages and thresholds.

other measurements. During this later testbeam, the majority of pixels had to be masked, as visible in the hitmap in figure 7.24. The reason for this has to be investigated. Even so, this highly irradiated

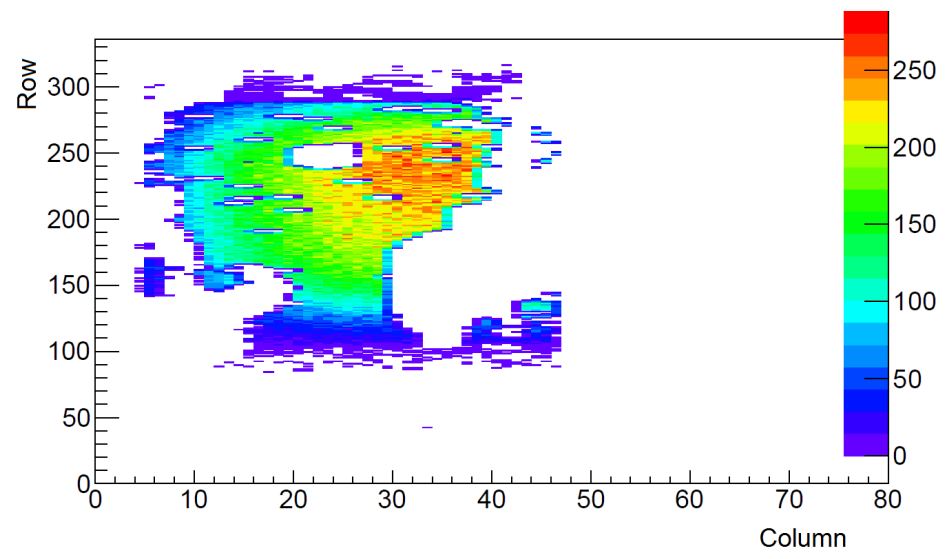

Figure 7.24: Hitmap of device FBK11 during June 2012 testbeam. Most of the sensor area is masked.

device showed an average hit efficiency of around $96 \%$ over a range of bias voltages at a threshold of $1500 \mathrm{e}^{-}$. The measured hit efficiencies for FBK11 at reasonable thresholds are in the region of the IBL requirement, at a threshold setting of $1800 \mathrm{e}^{-}$even beyond. The counter-intuitive efficiency drop at this threshold setting for rising bias voltages can be explained by the significant rise in leakage currents from $41 \mu \mathrm{A}$ at $140 \mathrm{~V}$ to $146 \mu \mathrm{A}$ at $150 \mathrm{~V}$ and finally $234 \mu \mathrm{A}$ at $160 \mathrm{~V}$, see table 7.6.

Devices SCC87 and SCC90 were investigated at high threshold values of $3200 \mathrm{e}^{-}$, which, as seen for 
other devices, has a negative impact on the hit efficiency for irradiated devices. The efficiency of around $93 \%$ might be caused by an irradiation to higher than $5 \cdot 10^{15} \frac{\mathrm{n}_{\mathrm{eq}}}{\mathrm{cm}^{2}}$.

\subsubsection{IBL Sensors - Summary}

Taking the results of a large set of lab measurements and the IBL testbeam campaigns, together with the 3D testbeam campaigns and planar testbeam campaigns, it was observed that both sensor technologies perform equally well and fulfil the requirements for the IBL detector. Consequently, the most aggressive design of the planar sensor candidates, the $200 \mu \mathrm{m}$ thick SE sensor, was chosen for the central $75 \%$ of the IBL staves. For the outer $25 \%$ of the staves, both, double sided 3D and full 3D sensors, are used, as shown in figure 7.25. Thus, the Insertable b-Layer is the first pixel detector system at CERN experiments, which uses a technology mixture. A photograph of the fully assembled IBL detector,

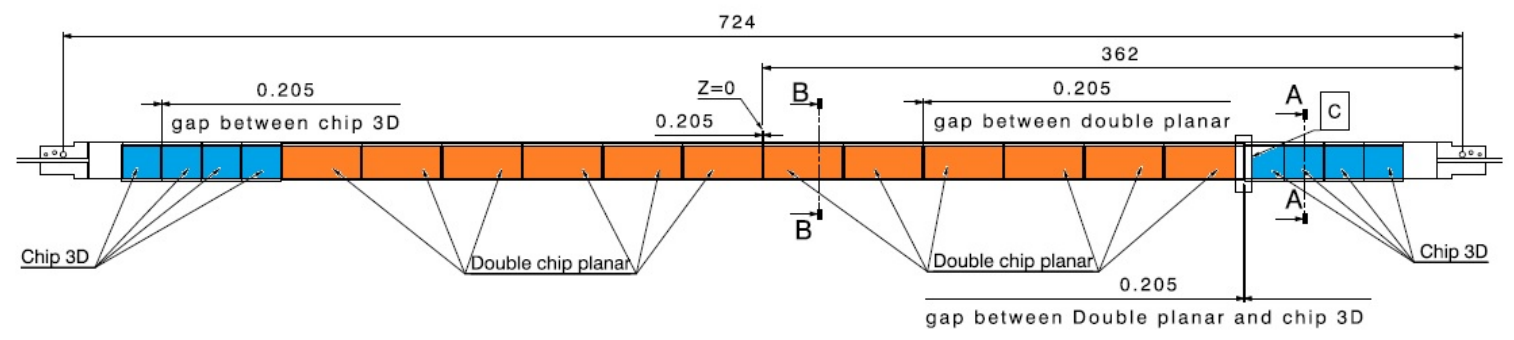

Figure 7.25: Stave layout for IBL, showing planar double chip sensors in the central 75\% of the stave and 3D sensors in the stave extremities. The design includes fixed gaps of $205 \mu \mathrm{m}$ between neighbouring modules. Taken from [46] and coloured for better visibility.

including services, is shown in figure 7.26.

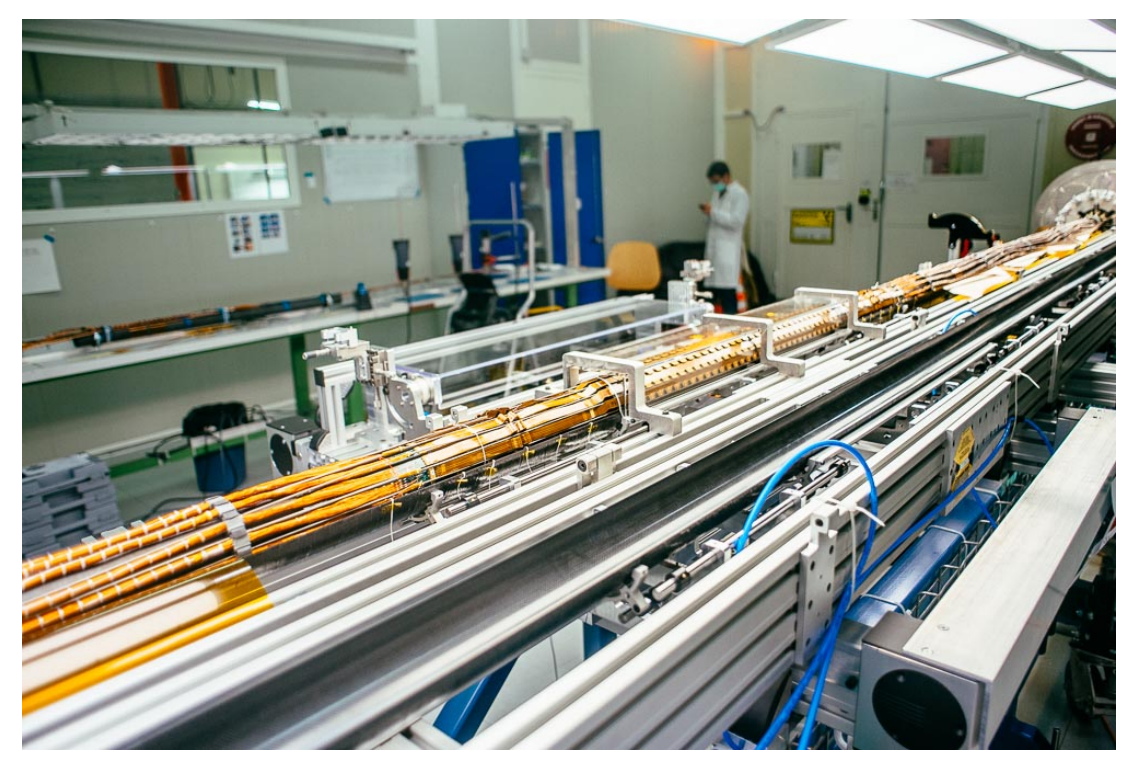

Figure 7.26: Photograph of the fully assembled IBL detector on a support structure, including services. The staves are protected by a plastic cover, which is kept in place by three metal clamps. Picture taken by H. Pernegger. 


\begin{tabular}{|c|c|c|c|c|}
\hline facility: CERN & runs: $50237-50253$ & used events: $1.8 \mathrm{M}$ & & \\
\hline & DUT 0 & DUT 1 & DUT 2 & DUT 3 \\
\hline sensor ID & SCC41 & SCC34 & SCC40 & SCC55 \\
\hline sensor design & PPS $250 \mu \mathrm{m}$ cons & 3D double sided & PPS $200 \mu \mathrm{m}$ SE & 3D double sided \\
\hline fluence $\left[10^{15} \frac{\mathrm{n}_{\mathrm{eq}}}{\mathrm{cm}^{2}}\right]$ & 0 & 5 & 0 & 0 \\
\hline threshold $\left[\mathrm{e}^{-}\right]$ & 3200 & 1600 & 1600 & 1600 \\
\hline ToT tuning & 8 ToT@20k & 8 ToT @ $20 \mathrm{ke}^{-}$ & 8 ToT @ $10 \mathrm{ke}^{-}$ & 8 ToT@ $@ 20 \mathrm{ke}^{-}$ \\
\hline bias voltage [V] & 100 & 140 & 100 & 20 \\
\hline angle in $\phi$ direction & $0^{\circ}$ & $0^{\circ}$ & $0^{\circ}$ & $0^{\circ}$ \\
\hline angle in $\eta$ direction & $0^{\circ}$ & $0^{\circ}$ & $0^{\circ}$ & $0^{\circ}$ \\
\hline average efficiency [\%] & 99.75 & 95.40 & 99.72 & 99.41 \\
\hline mean clustersize $X$ & 1.048 & 1.009 & 1.192 & 1.031 \\
\hline mean clustersize $Y$ & 1.236 & 1.099 & 1.153 & 1.271 \\
\hline
\end{tabular}

Table 7.7: IBL testbeam June 2011, batch 1. The different sensor design types conservative, 3D double sided, full 3D and slim edge (SE) are explained in section 4.2.

\begin{tabular}{|l|c|c|c|c|}
\hline facility: CERN & runs: 61500-61511 & used events: 2.6 M & & \\
\hline \hline & DUT 0 & DUT 1 & DUT 2 & DUT 3 \\
\hline sensor ID & SCC40 & LUB4 & FBK13 & CNM81 \\
\hline sensor design & PPS $200 \mu \mathrm{m} \mathrm{SE}$ & PPS $200 \mu \mathrm{m} \mathrm{SE}$ & full 3D & 3D double sided \\
\hline fluence $\left[10^{15} \frac{\mathrm{n}_{\mathrm{eq}}}{\mathrm{cm}^{2}}\right]$ & 0 & 4 & 0 & 5 \\
\hline threshold $\left[\mathrm{e}^{-}\right]$ & 3200 & 1600 & 1500 & 1500 \\
\hline ToT tuning & $8 \mathrm{ToT} @ 10 \mathrm{k}$ & 8 ToT @ $10 \mathrm{ke}^{-}$ & 8 ToT @ 20 ke & 8 ToT @ 20 ke \\
\hline bias voltage $[\mathrm{V}]$ & 150 & 1000 & 20 & 160 \\
\hline angle in $\phi$ direction & $0^{\circ}$ & $0^{\circ}$ & $0^{\circ}$ & $0^{\circ}$ \\
\hline angle in $\eta$ direction & $0^{\circ}$ & $0^{\circ}$ & $0^{\circ}$ & $0^{\circ}$ \\
\hline average efficiency $[\%]$ & 99.94 & 97.85 & 98.77 & 97.27 \\
\hline mean clustersize X & 1.033 & 1.016 & 1.028 & 1.010 \\
\hline mean clustersize Y & 1.187 & 1.130 & 1.190 & 1.081 \\
\hline
\end{tabular}

Table 7.8: IBL testbeam September 2011, batch 4 


\subsection{IBL Production Modules}

At the end of 2012 a final IBL testbeam campaign was performed at CERN, where a new set of modules was tested. These modules were taken from the final sensor production, of which all IBL modules were made. Between the devices tested in previous testbeams and the production modules, two main differences should be noted. The planar sensors of this final batch are all in the double chip slim edge design, described in section 4.2. The other major difference is that an enhanced version of the readout chip, the FE-I4B chip, was used for the production modules. Beside that, several monitoring functionalities and a temperature measurement circuit were added. A full list of features and changes can be found in [75]. The testbeam measurements required some changes in the data acquisition hard- and software, as well as in the track reconstruction and DUT analysis frameworks. The reason for these modifications were the tested planar production modules. While the tested 3D sensors had the same dimensions as in previous testbeams, the planar devices were produced as double chip sensors, being equipped with two FE chips. Thus, the data taking and track reconstruction had to be modified, such that the data from the two different chips was treated as one device under test. Furthermore, double chip sensors host three different pixel sizes, namely $500 \mu \mathrm{m}$ long pixels in the outermost columns 1 and 160,250 $\mu \mathrm{m}$ long pixels in columns 2 to 79 and 82 to 159 and as third type $450 \mu \mathrm{m}$ long pixels in column 80 and 81 . Columns 80 and 81 host pixels of this extended dimension to allow a gap between the mounted FE chips. The double chip sensor design gave the first impulses to set up the new DUT analysis framework TBmonII, with the possibility to describe and analyze several pixel types on one sensor. All presented results of IBL production modules and the larger sensors in section 7.7 were produced with a beta version of TBmonII and a preliminary, upgraded version of the track reconstruction framework.

The aim of the production module testbeam was to have a final functionality test of the production modules, equipped with the final version of the FE-chip. One of the most interesting measurements for the planar double chips was the efficiency analysis of the new $450 \times 50 \mu \mathrm{m}^{2}$ pixels in the centre of the sensor. Due to the tight schedule between the delivery of the production modules and the first necessary works on the IBL staves, only unirradiated production modules were tested. The feedback tuning of all tested devices during the October 2012 testbeam, was a response of $6 \mathrm{ToT}$ at a charge of $20 \mathrm{ke}^{-}$.

In the following, results from four different production module testbeam batches are presented. The measurement sets are sorted by device conditions and include results for planar double chip sensors, full 3D sensors and double sided 3D sensors. Only a fraction of the recorded data could be evaluated, for the rest of the dataset problems occurred during either track reconstruction or device analysis.

The first evaluated dataset was taken at a signal threshold of $3000 \mathrm{e}^{-}$for both devices and at perpendicular incidence. A bias voltage of $100 \mathrm{~V}$ was applied to the planar device and $25 \mathrm{~V}$ to the full 3D device. Figure 7.27 shows the efficiency maps for both tested devices, left the planar sensor, right the full 3D. The calculated average efficiencies are $98.89 \%$ for the planar chip and $98.87 \%$ for the full 3D. In both histograms only a fraction of the sensor area is filled with data points. As for the previous measurements the reason is, that tracks can only be matched in the overlap area of the scintillators. Since the scintillators are smaller than the investigated devices, only parts of the devices can be scanned. In the bottom part of both efficiency regions a drop to values of around $80 \%$ can be seen, which might be caused by a combination of an inclined installation of one of the scintillators and the slightly moving xy-table. Figure 7.28 shows an overview of the detected cluster charges for both devices, depending on the position on the sensor. For these histograms no match in the other devices is required, thus the full size of the beamspot is visible, but also noise hits. Since the buffers of both devices are only active after a trigger signal was received, the scintillators are visible in both histograms. An interesting result is that the cluster ToT distribution for the double chip sensor is homogeneous on one FE chip, but the transition between the two chips is clearly visible. Another very interesting feature of double chip devices is 

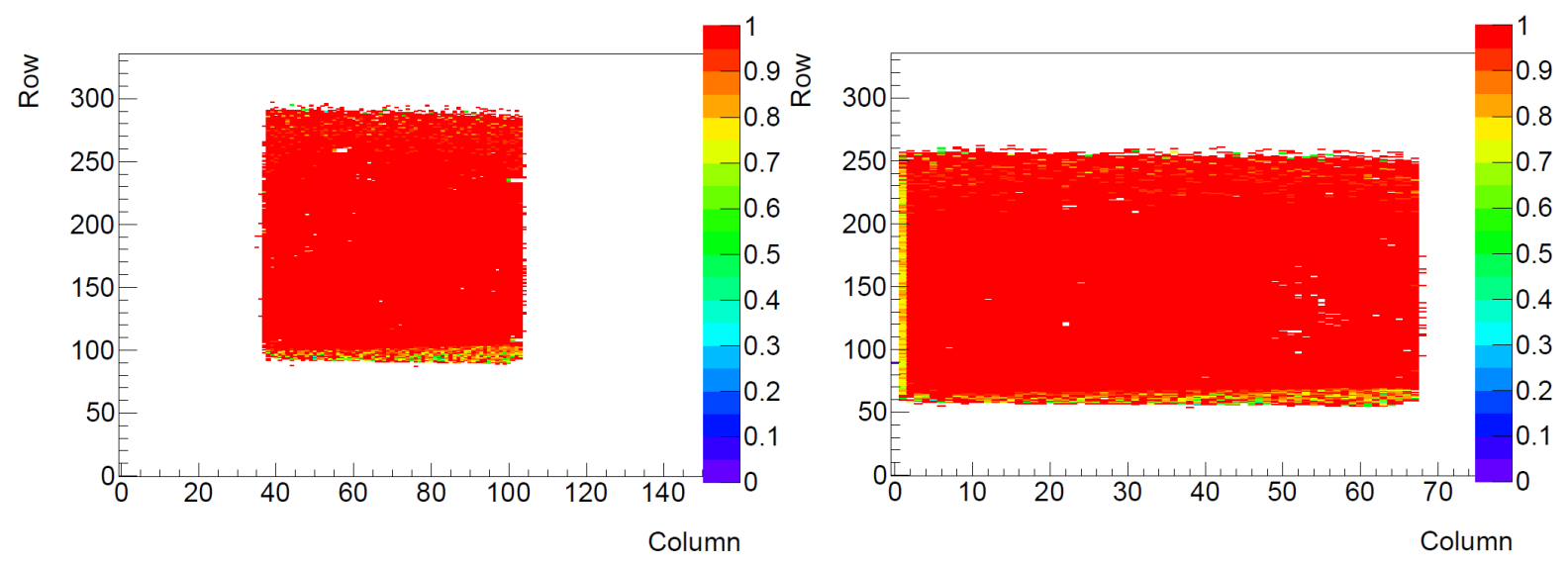

Figure 7.27: Efficiency map for a planar double chip (left) and a full 3D device (right) at perpendicular incidence. A signal threshold of $3000 \mathrm{e}^{-}$was applied to both devices and bias voltages of $100 \mathrm{~V}$ for the planar device and $25 \mathrm{~V}$ for the full 3D. Both devices show a homogeneous and high efficiency over the whole sensor.
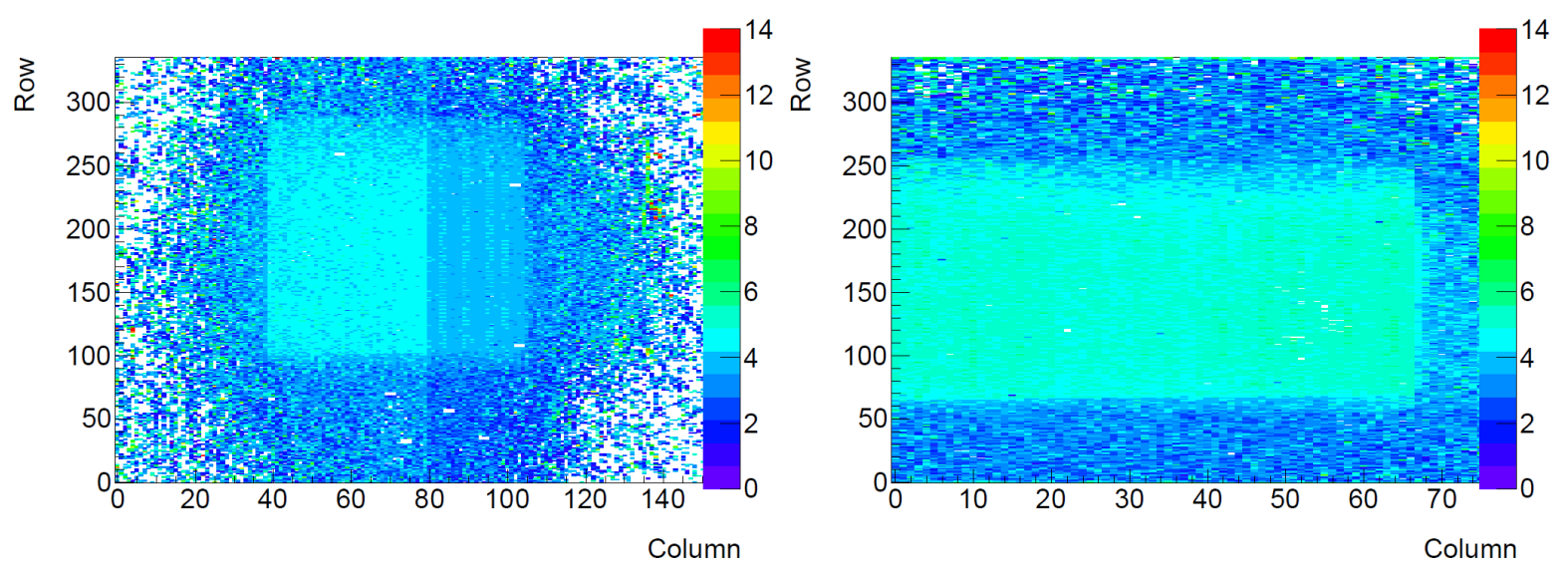

Figure 7.28: Average detected cluster ToT value, depending on the position on the sensor. The planar double chip is shown on the left, the full 3D device on the right. For both devices the scintillator area is clearly visible.

shown in figure 7.29. Both histograms show the average time between the trigger signal was issued and a matched cluster was detected in units of $25 \mathrm{~ns}$. The double chip clearly shows different response times for the left and the right chip, the response time within the single chip 3D device is homogeneous. This can likely be compensated in the settings of the FE chips, but further studies are necessary.

The same devices were measured afterwards at a signal threshold of $2000 \mathrm{e}^{-}$and rotated by $15^{\circ}$ with respect to the previous position. The bias voltages were again $100 \mathrm{~V}$ for the planar device and $25 \mathrm{~V}$ for the full 3D. Again, as first analysis an efficiency measurement was performed and the result is shown in figure 7.30. As the histogram already indicates, the efficiency measurement for the planar device was as expected (average efficiency $98.76 \%$ ), while there were problems with the 3D device (average efficiency $86.80 \%$ ). Further investigations showed that during the analyzed runs a further reference device was included in the data taking, which temporarily caused problems for some runs. This assumption can be confirmed by analyzing the average hit efficiency as a function of the run number, as shown in figure 7.31. As the histograms clearly show, the planar double chip was not affected by the problems, while for some runs the 3D efficiency clearly drops, which is subject of further investigations. Since 

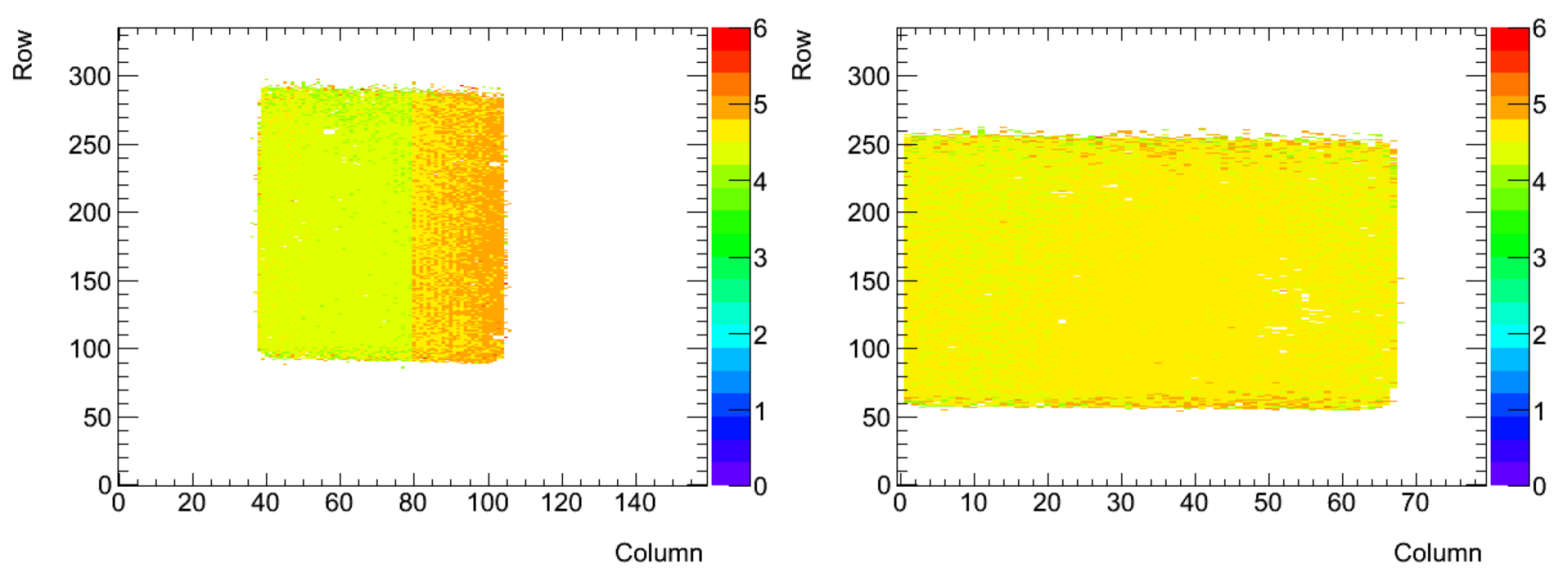

Figure 7.29: Average time between a trigger signal is sent and a matched cluster is detected in a device in units of $25 \mathrm{~ns}$ for a planar double chip device (left) and a full 3D device (right) at a threshold of $3000 \mathrm{e}^{-}$. This is a result of slightly different feedback tuning of the two FEs.
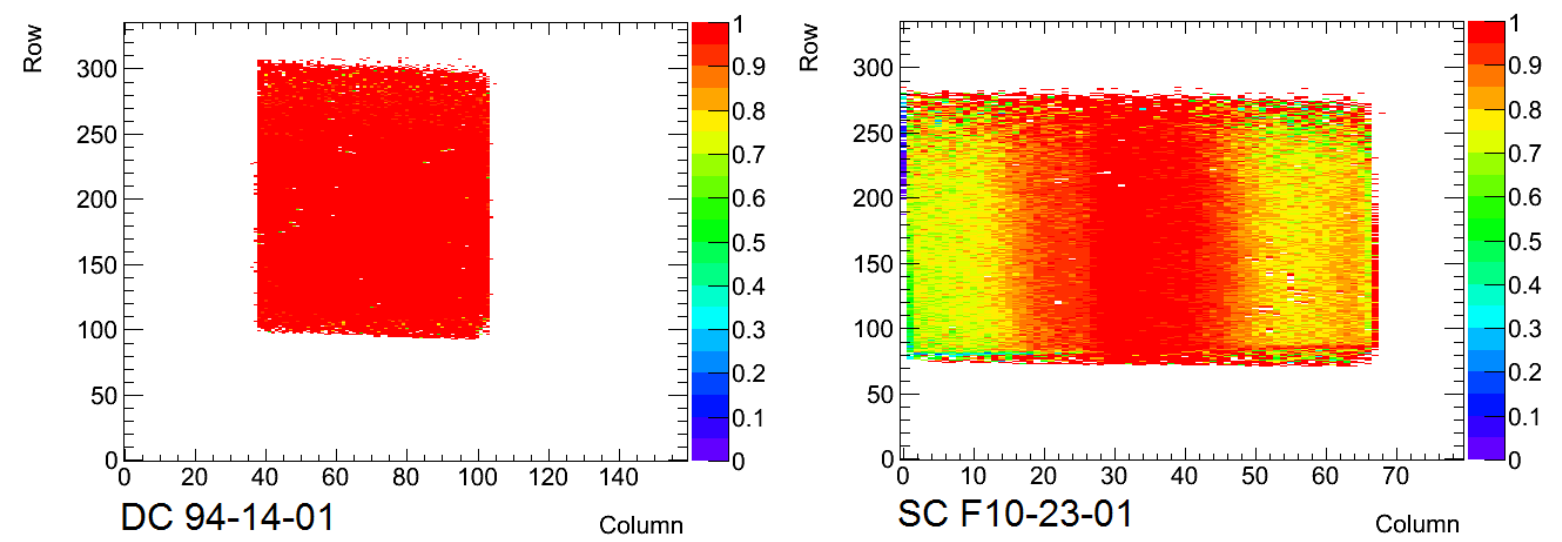

Figure 7.30: Efficiency map for a planar double chip (left) and a full 3D device (right), rotated by $15^{\circ}$ along $\phi$-direction. The signal threshold was set to $2000 \mathrm{e}^{-}$, the bias voltages were $100 \mathrm{~V}$ for the planar device and $25 \mathrm{~V}$ for the full 3D.

the double chip sensor was positioned, such that the beam spot hits the centre of the device, the efficiency of the central double columns of the planar chips could be analyzed. As figure 7.32 shows, the hit efficiency of the $450 \mu \mathrm{m}$ long pixels is sufficient and no drop towards the edges can be observed, which corresponds to the expectation based on the measurements of edge pixel efficiencies at earlier testbeams. The visible patterns in the histograms can be explained by the comparably low statistics due to the small sensor area under study. The obtained result is also consistent with a previously performed measurement with a modified version of the first TBmon version.

Due to the data taking problems with the 3D device, the devices were shifted to other positions and the data taking was restarted under the same parameters again, including the reference device. The resulting efficiency maps for the double chip and the full 3D device are shown in figure 7.33. The effects seen before, caused by problems with the data taking, are not visible anymore. In this set of measurements, both devices perform as expected and show average hit efficiencies of 97.25\% (planar double chip) and $97.87 \%$ (full 3D). 

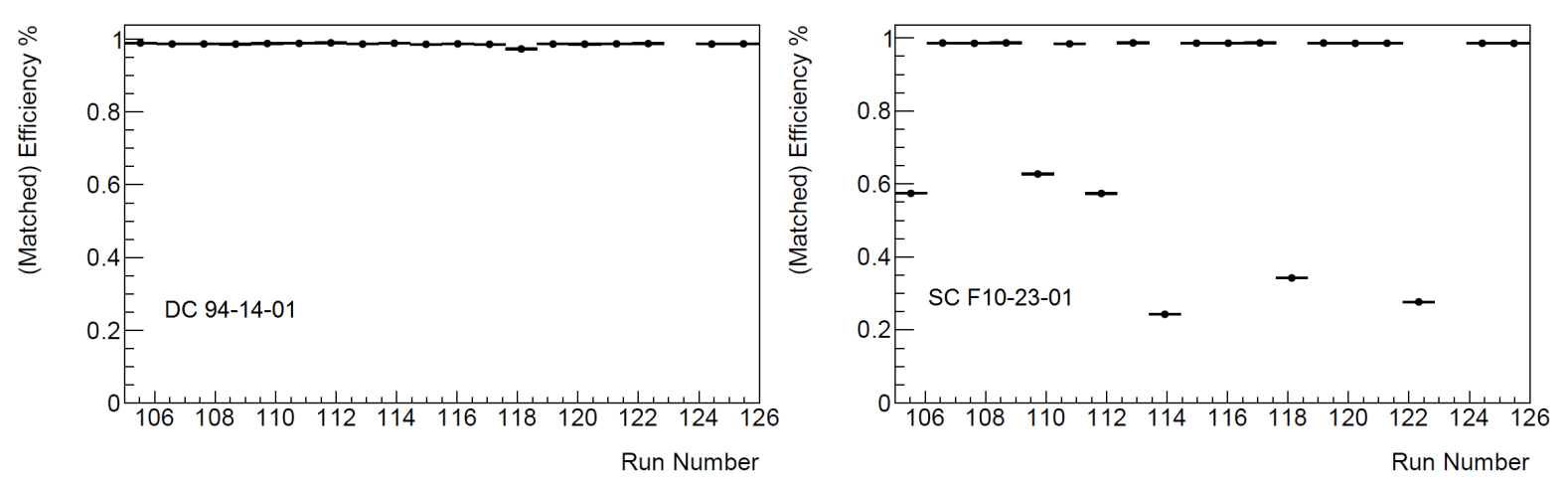

Figure 7.31: Average hit efficiency as a function of the run number for the planar device (left) and 3D device (right). The data taking problems, which influenced the $3 \mathrm{D}$ device in some runs, can be clearly seen in the calculated efficiency.

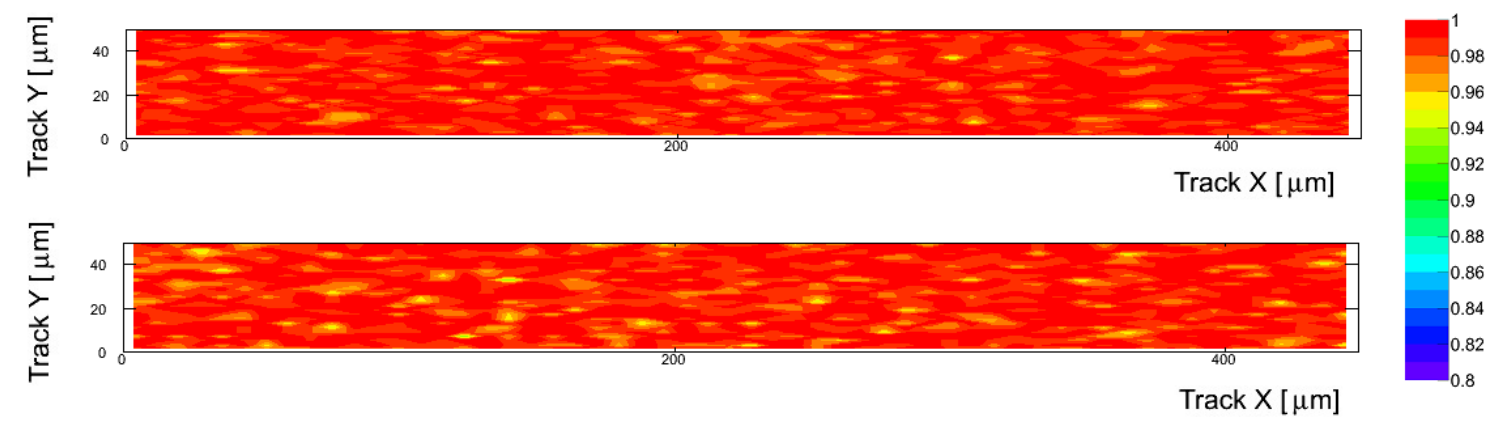

Figure 7.32: Hit efficiency for the pixels in the central double column of the double chip sensor. Top: measurement at a threshold of $3000 \mathrm{e}^{-}$and perpendicular incidence. Bottom: measurement at a threshold of $2000 \mathrm{e}^{-}$, device rotated by $15^{\circ}$. The bias voltage was $100 \mathrm{~V}$ for both measurements. For both measurements the preliminary hit efficiency is above $98 \%$.
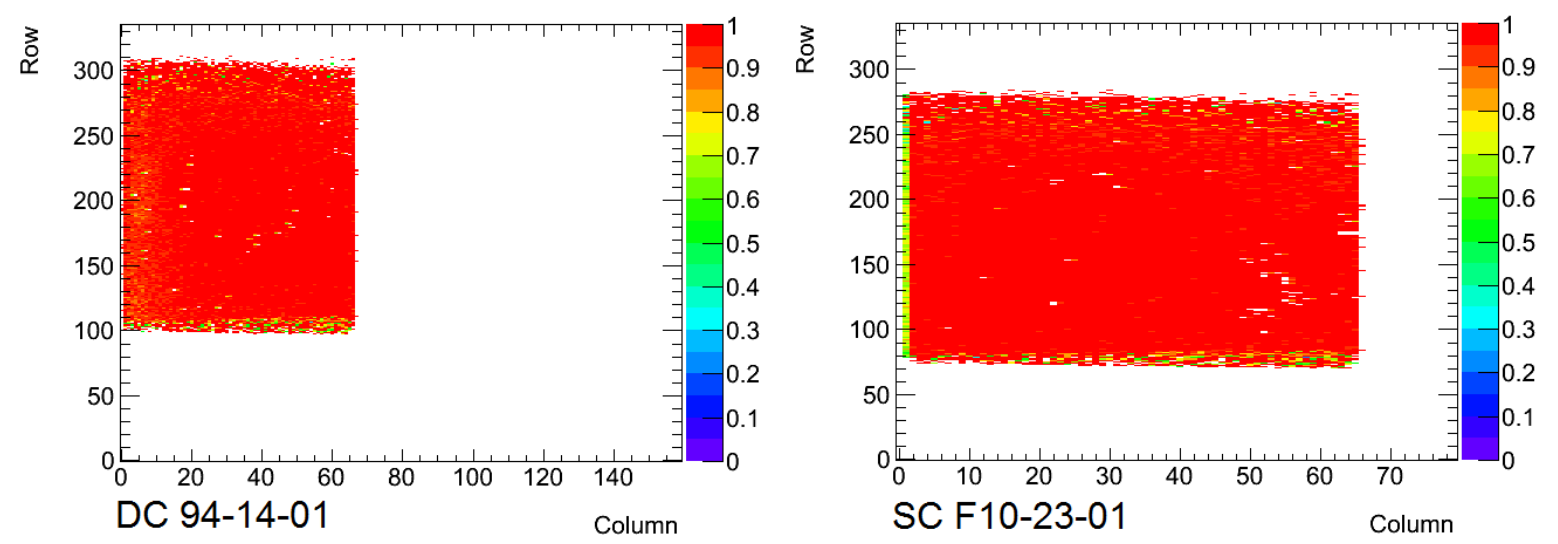

Figure 7.33: Second set of measurements at a rotation of $15^{\circ}$, threshold of $2000 \mathrm{e}^{-}$and bias voltages of $100 \mathrm{~V}$ for the planar device (left) and $25 \mathrm{~V}$ for the full 3D (right). The previous data taking problem was solved and both devices show the expected high efficiencies over the whole region, covered by the scintillators. 
The last batch of runs that could be reconstructed and analyzed with the current versions of EUTelescope and TBmonII was taken with another planar double chip sensor and a double sided 3D device. Bias voltages of $100 \mathrm{~V}$ were applied to the planar sensor and $20 \mathrm{~V}$ to the 3D sensor. Again, both devices were tilted by $15^{\circ}$ with respect to perpendicular incidence along the $\phi$-direction. The efficiency maps for both devices are shown in figure 7.34. The average hit efficiency is $97.49 \%$ for the planar device $92-$
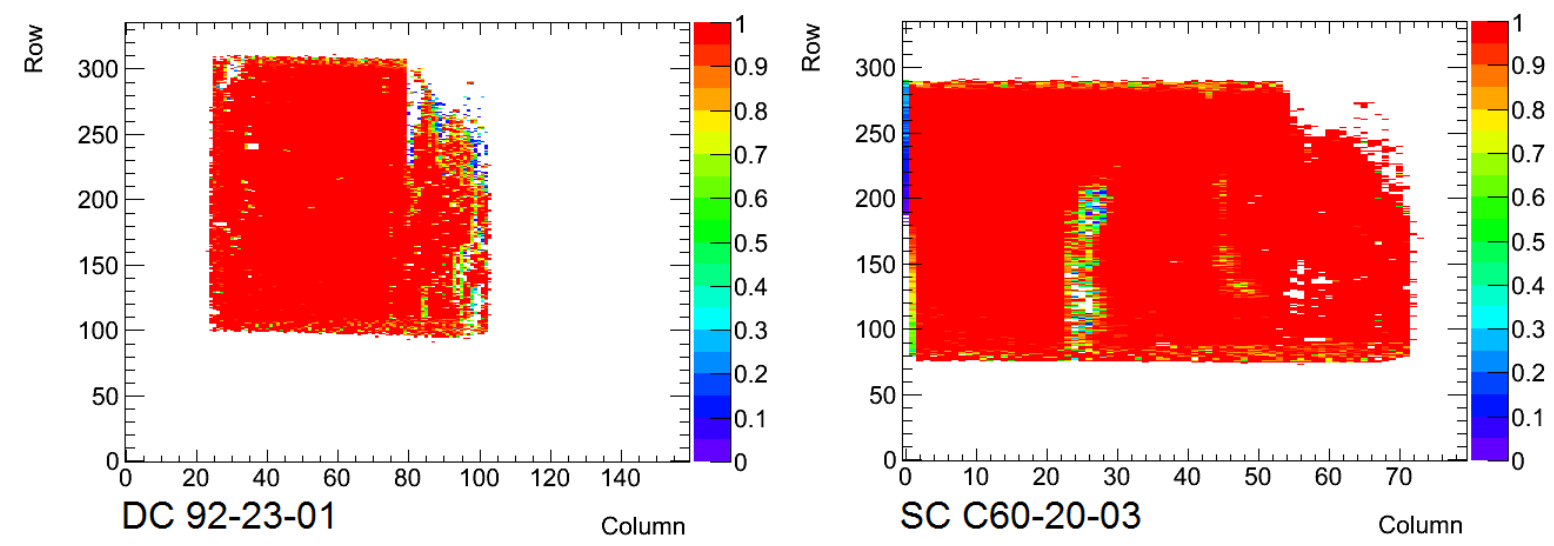

Figure 7.34: Efficiency measurement at a rotation of $15^{\circ}$, threshold of $3000 \mathrm{e}^{-}$and bias voltages of $100 \mathrm{~V}$ for the planar device (left) and $20 \mathrm{~V}$ for the double sided 3D (right).

23-01 and 98.18\% for the full 3D device C60-20-03. As visible in the efficiency maps, for both devices a fraction of the sensitive area is without any information. The same can be seen in the raw hitmaps in figure 7.35 , where the same structures can be observed. The reasons behind these empty regions in both
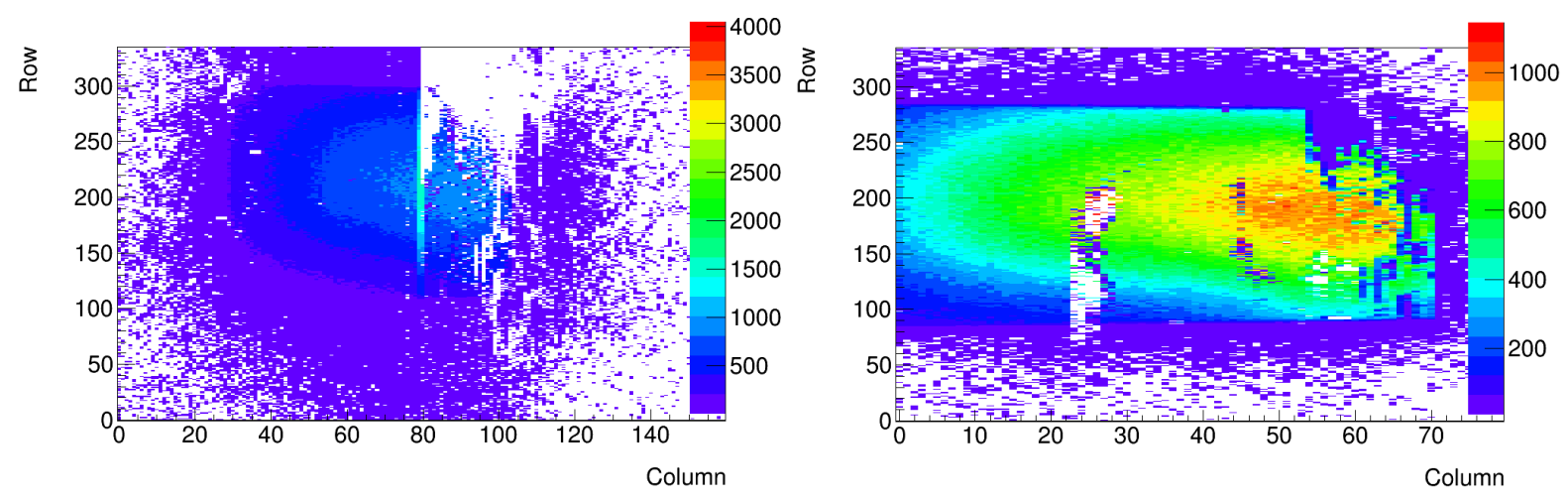

Figure 7.35: Raw hitmaps for double chip 92-23-01 (left) and double sided 3D device C60-20-03 (right). The regions free of hits correspond to the empty areas in the efficiency maps.

sensors might be disconnected bump bonds, but have to be examined further.

During the IBL production module testbeams, the modules showed the expected average efficiencies. Especially the long pixel double columns did not show any signs of reduced hit efficiency towards the outer edges of the pixels. The final data analysis for these runs has to be performed with the new reconstruction software. This requires an updated version of the reconstruction software, including the possibility to model sensors with several different pixel geometries. 


\subsection{Large Sensors for HL-LHC}

As already briefly described in section 4.1.2, future upgrades of the ATLAS Inner Detector are planned. During the upgrade phase in around 10 years, a full replacement of the Inner Detector is foreseen. This upgrade will include a new pixel detector with a total sensor area on the order of $8 \mathrm{~m}^{2}$. Such huge sensor areas require approaches that are more cost efficient than current sensor productions. Since a large fraction of the production costs are caused by handling work required for the bump-bonding process, one approach to lower costs is to produce larger sensors. This would lower the handling costs per area and therefore also significantly lower the costs of the final detector. The planar pixel sensor community decided to produce 4-chip sensors as a first approach towards larger devices. Here a 4-chip sensor is taken from a wafer on which two planar IBL double chip sensors are located next to each other and instead of separating the two double chips, they are used as one sensor. Four FE-I4 chips are bonded to the sensor, resulting in a 4-chip module. Of course, this way of production can only be taken as a first approach towards large sensors, since it includes a dead region of around $1.5 \mathrm{~mm}$ between the top and bottom double chip sensor, which is usually the region where the wafer is cut.

During the planar pixel sensors testbeam in August 2013, different 4-chip sensors were tested. In the following, results of a device produced by MPP Munich and University of Göttingen are shown. The device has a thickness of $200 \mu \mathrm{m}$ and a total size of $40.4 \times 34.0 \mathrm{~mm}^{2}$. Due to the mentioned horizontal gap in the sensor design and the same pixel types and dimensions as for planar double chip sensors, the track reconstruction is preliminary, since the geometry description does not allow gaps. For the preliminary reconstruction a workaround was implemented. A proper description of all sensor features in TBmonII is possible, which allowed to get a first impression of the hit efficiency.

A bias voltage of $120 \mathrm{~V}$ was applied to the 4-chip sensor for this measurement. The device was tuned to a signal threshold of $3200 \mathrm{e}^{-}$and a charge response of $8 \mathrm{ToT}$ at $16 \mathrm{ke}^{-}$. As shown in figure 7.36, the hit efficiency of the 4-chip device is homogeneous over the whole active area. On the right, the
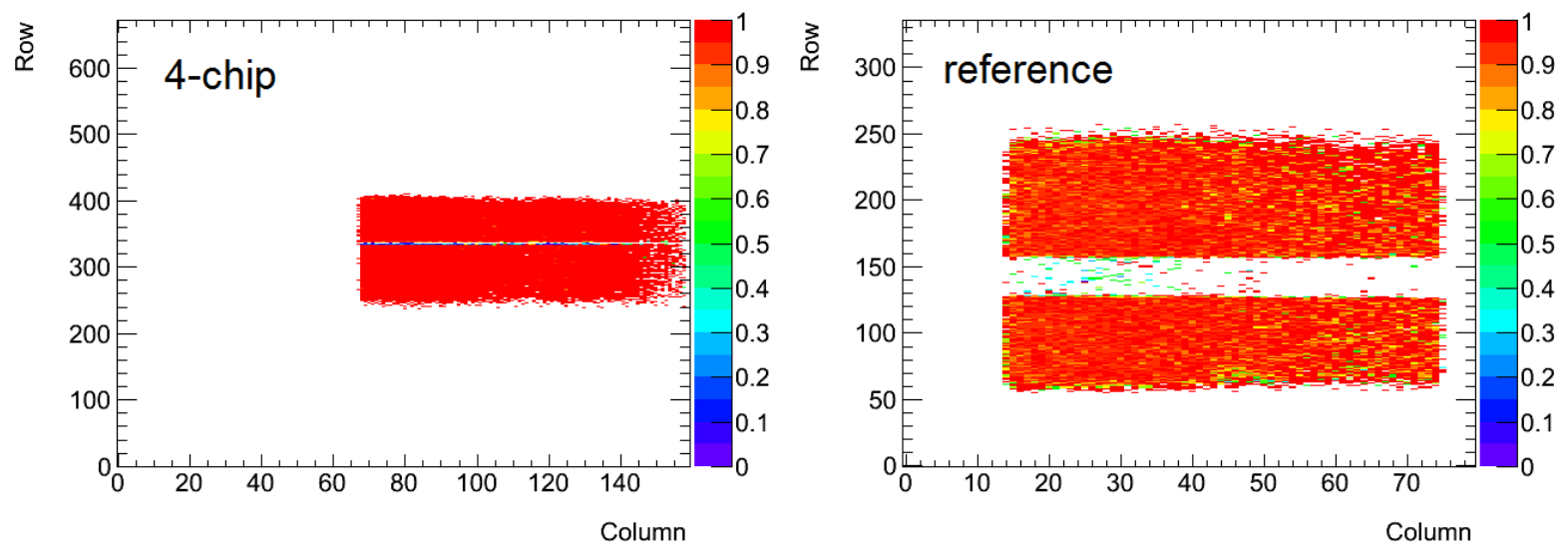

Figure 7.36: Efficiency maps for the investigated 4-chip device (left) and the reference device (right). The 4-chip device shows a homogeneous efficiency with a small horizontal gap in the region, where the lower double chip ends and the upper double chip begins. In the efficiency map of the reference device the same gap can be seen around row 150 in full size.

hit efficiency of the reference device is shown. In the region around row 150, the gap in the efficiency map can be seen clearly, having a height of about 30 rows. Taking into account the pixel dimension of $50 \mu \mathrm{m}$ in row-direction, the visible gap matches the previously mentioned gap in the sensor design of $1.5 \mathrm{~mm}$. The few visible artefacts in the gap region of the efficiency map are caused by the not perfect 
track reconstruction, which requires a very large matching radius for the analyses. As preliminary result, average hit efficiencies of $99.18 \%$ for the 4-chip device and $93.51 \%$ for the reference device were calculated.

Since the double chip modules showed the interesting feature of having different response times, depending on the FE chip they are detected in, the same analysis was performed for the 4-chip device. As shown in figure 7.37, the same feature is found for 4-chip modules. The earliest response time is
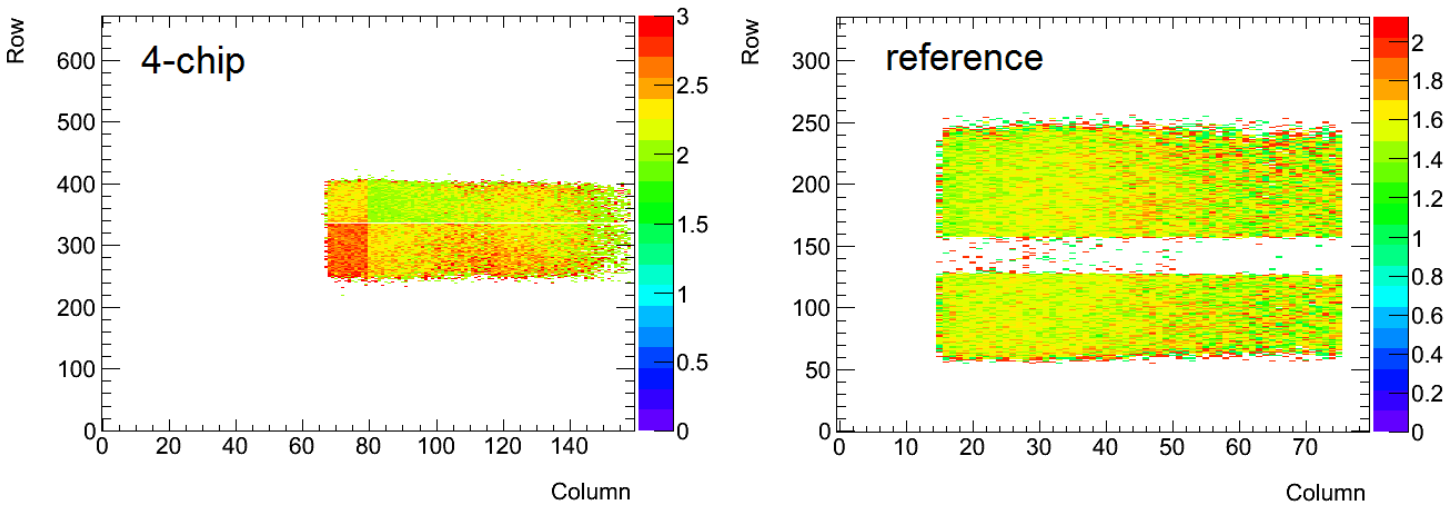

Figure 7.37: Average delay between a trigger signal is sent and a matched cluster is detected in a device in units of 25 ns. Left: the planar 4-chip device shows different average response times, depending on the FE chip in which the signal is processed. Right: the reference single chip device shows a homogeneous response time. Both devices were tuned to a threshold of $3200 \mathrm{e}^{-}$.

measured for the FE chip in the upper right, at around 1.5 LVL1 - units, where each unit corresponds to $25 \mathrm{~ns}$. The two FE chips on the diagonal have the same response delay of around 2.3 LVL1 - units and the lower left chip the longest response time of around 3 LVL1 - units. This different response behaviour for devices equipped with more than one FE chip should be further investigated.

Further approaches of the planar pixel sensors community towards future ATLAS upgrades can be found in [76]. 



\section{Chapter 8}

\section{Summary and Outlook}

\subsection{Summary}

The current long shutdown of the LHC is used by the ATLAS experiment to install a fourth pixel detector layer, the Insertable b-Layer (IBL), inside the current pixel detector. Since this new detector will be placed on a new smaller beampipe, it will be located closer to the interaction point than any other sensitive material in ATLAS. Due to its position in the experiment, the IBL layer has to withstand higher fluences and particle rates than the current innermost pixel layer. This harsh environment required sensor designs that are more radiation tolerant. To cope with the high expected hit occupancy per area, the pixel pitch was reduced from $400 \mu \mathrm{m} \times 50 \mu \mathrm{m}$ to $250 \mu \mathrm{m} \times 50 \mu \mathrm{m}$. As candidate designs, planar silicon, double sided 3D silicon and full 3D silicon sensors were investigated by the IBL community. A set of quality criteria was defined at the beginning of the project, which the designs had to fulfil in lab and testbeam measurements, to be considered as potential sensor for the IBL.

All testbeam measurements performed by the IBL community were done at the CERN and DESY sites, using the EUDET beam telescope and its copies. In the testbeam campaigns, the hit efficiencies before and after irradiation at different operation parameters, the active edge areas and the collected signals in thin sensors were amongst the measurements with highest priority. While the planar sensor designs were an evolution of the current ATLAS pixel sensor, the 3D approach was considered for the first time as sensor concept in a high energy physics experiment.

The EUTelescope framework was used to evaluate the testbeam measurements and expanded for the ATLAS pixel requirements and measurements. The USBpix and RCE readout systems were integrated into the data acquisition chain and the reconstruction software was structured, such that after a five step procedure a root file is produced, which contains all relevant information of the devices under test.

The final analyses were performed in TBmon, a standalone tool designed for testbeam analysis of ATLAS pixel devices. Intentionally, TBmon was developed as online monitor for testbeams of the 3D sensor community. After the first IBL testbeam, first modifications were realized, which made TBmon an offline analysis tool, that was used for all IBL testbeam analyses. Furthermore, TBmon became the standard analysis tool for all testbeams of the planar pixel sensor community, 3D sensor community and diamond sensor community.

The results obtained from these testbeam campaigns were an important contribution for the final sensor decision for the IBL project. Since the slim edge planar design, the double sided 3D design and the full 3D design all showed satisfying results, it was decided to build IBL as a mixed technology project. The central $75 \%$ of the detector layer are planar sensors, the outer $12.5 \%$ on either side are double sided 3D and full 3D sensors. To gain a higher geometric efficiency, the final planar sensor design was realized as double chip modules with extended pixel cells in the central double column, where each sensor is read out by two FE-I4 readout chips.

In parallel to the developments towards IBL, the sensor communities started developments for future detector upgrades, especially the upgrade for phase 2 in the year $\sim 2023$, where the whole ATLAS Inner 
Detector will be replaced. As a first approach towards the development of more cost efficient devices, the planar pixel community produced 4-chip modules, where each sensor has the size of four FE-I4 chips. In 2013, the first 4-chip modules were tested in testbeam measurements at DESY, using again the EUDET telescope.

Due to the design of the planar double chip modules, which have three different pixel types per sensor, and the 4-chip prototype modules, which in addition have an inactive horizontal gap in the design, it was necessary to develop a new version of the testbeam analysis tool TBmon. The design of TBmon only allowed a proper geometry description of sensors with one pixel type in the central sensor region and one additional type of edge pixels. The design of the new software version TBmonII includes a completely new structure of the software core, which allows to describe sensors with several different pixel types per device. With TBmonII, the analysis of the IBL planar double chip modules and 4-chip modules was possible. Due to the very flexible geometry description in TBmonII, the framework can also be used for analysis of future (new) sensor designs, even for designs without a regular order.

\subsection{Outlook}

The studies of IBL modules presented in this thesis offer a rather complete image of the performance of the modules. However, due to the very tight schedule for IBL installation no measurements could be done with modules irradiated to the end of lifetime fluence. This should be done in the near future.

Since testbeam measurements are a crucial part of the development of new sensors, updating the reconstruction and analysis software frameworks will be very important also in the future. For the phase 2 upgrade the sensor groups start looking at various different layouts for smaller pixels to improve track separation and vertexing performance for the future tracker. This requires a more flexible implementation of the geometry description, as well as adaptations of clustering and fitting algorithms, as started in the development of TBmonII. Also the study of features like the different response times in double chip and 4-chip devices require constant updates of the analysis procedures, showing that a lot of work remains to be done in the field of testbeam data reconstruction and analysis. 


\section{Acknowledgements}

I want to thank everyone who supported me during the last years and especially the last months and made it such an exciting time. The group of people who I want to express my gratitude to, is probably too large to list every name here, so I want to say a big THANK YOU to everyone who is not explicitly mentioned here.

My first and biggest gratitude belongs to my parents and my sisters, who were there for me in every situation of my life. Without you nothing of this would have been possible! Thank you so much for all your support, patience and for always giving me the freedom to do whatever I want!

I also want to thank my supervisors Jörn Große-Knetter, Arnulf Quadt and Jens Weingarten for not only leading me towards the surely fascinating, but for beginners also confusing world of testbeams, but for guiding me along the right path! Thank you for all the interesting, funny and sometimes even crazy times, for sharing your knowledge with me and for supporting me in my work whenever it was necessary! And of course special thanks for the delicious espresso after lunch! Once more: thanks a lot Jens, for proof-reading my thesis and your "quiet dignity and grace". Long live the Beratungsstelle!

Of course I want to thank the members of our institute, for giving me such a nice time during working hours and beyond. Special thanks go to Boris "Glamour" Lemmer [77] for always being the nice guy and the helping hand in the institute and for all the crazy ideas! Furthermore, I want to explicitly mention Julia Rieger, Lars Graber, Johannes Agricola and the other hardware guys for the nice atmosphere and for giving me the feeling to not only work with colleagues, but with friends.

Thanks a lot to all the people from the 3D, IBL and planar community for all the testbeam adventures! Honestly, I must say: I start missing those times! Special thanks go to aunt Ingrid, Bane, Clara, Georg, Jenny, Igor, Mathieu, Slava and all the others! Of course I want to thank my friends Silke Altenheiner and Malte Backhaus for all the fruitful discussions on Skype and in person.

I want to thank all my friends, who are a big part of my life. I know I can count on you whenever it is necessary and I want to thank you for helping me to relax and get my mind off the work from time to time. Here I especially want to thank Stephan Semmelroth, Tim Ellringmann and my former flatmates Desiree Schreiber and Boris Hagedorn.

My very special gratitude goes to Chiara Meroni for giving me the advice: "you should go to that Italian language course for $\mathrm{PhD}$ students"! Thank you Chiara, that was the best advice I ever received! This brings me to my final words. Leila, thank you for all your love and support, for making me laugh, for sometimes confusing me, for making me smile so often and for giving me all that happiness!

When reading all these words, maybe it gives the impression that I really have a wonderful life and the best is: that is absolutely true! Thank you to all of you!! I want to conclude with a general advise, that can probably also help others and that should be noted down:

"Irgendwatt is halt immer!" [78] 



\section{Appendix A}

\section{Useful information}

\section{A.1 Testbeam Dictionary}

\begin{tabular}{|c|c|}
\hline testbeam language & meaning \\
\hline upstream & direction along the beam axis, pointing towards the beam source \\
\hline downstream & $\begin{array}{l}\text { direction along the beam axis, pointing in the flight direction of } \\
\text { the particles }\end{array}$ \\
\hline DUT & device under test (in our case: ATLAS pixel devices) \\
\hline reconstruction & $\begin{array}{l}\text { determining the track of each single particle through the sensor } \\
\text { planes, incl. "cluster selection", based on HotPixelKiller, Align- } \\
\text { ment, etc (in our case: EUTelescope) }\end{array}$ \\
\hline analysis & $\begin{array}{l}\text { analysis and plotting of properties, using the reconstruction out- } \\
\text { put (in our case: tbmon) }\end{array}$ \\
\hline hit & $\begin{array}{l}\text { a single pixel in coordinates of (col/row), which has detected a } \\
\text { signal }\end{array}$ \\
\hline cluster & group of hits, which were caused by the same incident particle \\
\hline track & $\begin{array}{l}\text { trajectory of a particle through the whole setup (scintillators, tele- } \\
\text { scope planes, DUT planes) }\end{array}$ \\
\hline trigger & $\begin{array}{l}\text { readout signal, sent to all connected devices (telescope and DUT } \\
\text { planes), caused by scintillator coincidence }\end{array}$ \\
\hline scintillators & $\begin{array}{l}\text { part of the testbeam setup; one pair at } 90^{\circ} \text { to each other upstream, } \\
\text { one pair downstream; coincidence of all four scintillators fires a } \\
\text { trigger }\end{array}$ \\
\hline event & $\begin{array}{l}\text { all data belonging to one trigger; read out buffer length of the } \\
\text { APIX devices: } 16 \mathrm{LVL} 1(=16 \times 25 \mathrm{~ns}) \text {, read out latency of tele- } \\
\text { scope planes } \approx 120 \mu \mathrm{s}\end{array}$ \\
\hline run & $\begin{array}{l}\text { data set, typically consisting of } 100 \mathrm{k} \text { events, all taken under the } \\
\text { same conditions }\end{array}$ \\
\hline measurement block & $\begin{array}{l}\text { set of runs, taken with the same setup under same conditions } \longrightarrow \\
\text { data can be combined for analysis }\end{array}$ \\
\hline plane & telescope or APIX device \\
\hline sensor ID & $\begin{array}{l}\text { identifier for the planes; for telescope }[0, \ldots, 5] \text {, for FE-I3 devices } \\
{[10, . ., 19] \text {, for FE-I4 devices }[20, . ., 29]}\end{array}$ \\
\hline ToT & $\begin{array}{l}\text { time over threshold; bit coded value for charge deposited in a } \\
\text { pixel cell }\end{array}$ \\
\hline residuals & distance between measured and extrapolated track \\
\hline
\end{tabular}




\begin{tabular}{|c|c|}
\hline track candidate & $\begin{array}{l}\text { high enough number (typ.: in four telescope and one DUT planes) } \\
\text { of hits/clusters along a narrow region around the extrapolated } \\
\text { particle trajectory }\end{array}$ \\
\hline track & description of trajectories of particles, based on measured hits \\
\hline reference plane & $\begin{array}{l}\text { typically a well known devices, in our case used for time resolu- } \\
\text { tion of detected hits }\end{array}$ \\
\hline in-time hits & detected hits which arrived within the specified LVL1 range \\
\hline alignment & $\begin{array}{l}\text { track based measurement of sensor positions, producing a collec- } \\
\text { tion containing shifts and rotations }\end{array}$ \\
\hline broken tracks & $\begin{array}{l}\text { fitted tracks for which not all planes detected a hit and/or the } \\
\text { tracks are kinked }\end{array}$ \\
\hline matched tracks & $\begin{array}{l}\text { track candidates that can be matched to a hit within the matching } \\
\text { radius (typ. } 1.5 \text { times pixel pitch) }\end{array}$ \\
\hline eta correction & $\begin{array}{l}\text { correcting cluster positions, taking into account the ToT values of } \\
\text { the participating hits }\end{array}$ \\
\hline tbtrack file & $\begin{array}{l}\text { root file produced by reconstruction, containing raw hits, clusters } \\
\text { and extrapolated track positions }\end{array}$ \\
\hline matched plane & $\begin{array}{l}\text { plane containing a hit that can be matched to a track in the invest- } \\
\text { igated event }\end{array}$ \\
\hline neighbouring hit & $\begin{array}{l}\text { either direct or diagonal neighbouring pixel; important for cluster } \\
\text { search }\end{array}$ \\
\hline LVL1 window & $\begin{array}{l}\text { specified part of the LVL1 range; all hits with a timestamp out of } \\
\text { this window are ignored }\end{array}$ \\
\hline hot pixels & $\begin{array}{l}\text { pixels detecting lots of noise hits, typically with an occupancy of } \\
\text { more than } 5 \cdot 10^{-3}\end{array}$ \\
\hline split cluster & $\begin{array}{l}\text { typically large clusters, containing a masked pixel; as con- } \\
\text { sequence the large cluster is artificially split into two seperate } \\
\text { clusters }\end{array}$ \\
\hline edge & $\begin{array}{l}\text { all pixels in the outermost column(s); in some designs larger than } \\
\text { "standard" pixels }\end{array}$ \\
\hline OnlineMonitor & $\begin{array}{l}\text { tool to display some preliminary live-information during data tak- } \\
\text { ing }\end{array}$ \\
\hline processor & $\begin{array}{l}\text { one of the five reconstruction steps (converter, clustering, hit- } \\
\text { maker, align, fitter) }\end{array}$ \\
\hline de-synchronization & shift in trigger IDs between telescope and DUT data stream \\
\hline mask & $\begin{array}{l}\text { list of pixels that were found to be hot or dead; these are masked } \\
\text { e.g. for the alignment step }\end{array}$ \\
\hline converter & data conversion from raw format to LCIO format \\
\hline clustering & cluster search processor, see "cluster" \\
\hline LVL1 ID & timestamp of an APIX hit in an event in units of LVL1s $[0, . ., 16]$ \\
\hline shifts/translations & alignment corrections in $\mathrm{x}-\mathrm{y}$-direction \\
\hline rotations & $\begin{array}{l}\text { orientation of a device, described by a rotation matrix (for } 90^{\circ} \\
\text { rotations) and on top given rotation angles around the three axes }\end{array}$ \\
\hline correlations & $\begin{array}{l}x-\text { or } y-p o s i t i o n \text { of a hit in one device, compared to the corres- } \\
\text { ponding hit position in another device }\end{array}$ \\
\hline
\end{tabular}




\begin{tabular}{|l|l|}
\hline global coordinate system & $\begin{array}{l}\text { Euclidean coordinate system produced by hitmaker, to describe } \\
\text { the whole setup taking the center of the first telescope plane } \\
\text { (sensor ID 0) as }(0,0,0) \text { position }\end{array}$ \\
\hline Distance Max & $\begin{array}{l}\text { opening size of a cone within which track candidates are being } \\
\text { searched for; given in units of um per 10 cm z-distance }\end{array}$ \\
\hline Kalman filter & $\begin{array}{l}\text { recursive approach to estimate value of state vector (here: state } \\
\text { vector = particle track) }\end{array}$ \\
\hline DAF fitter & $\begin{array}{l}\text { using Kalman filter approach, but reweighting measurements } \\
\text { (match candidates) until probability of one solution exceeds a pre- } \\
\text { defined value }\end{array}$ \\
\hline preprocessing & $\begin{array}{l}\text { data preparation including hotpixel finder, checkalign (tbmon } \\
\text { alignment) and track builder }\end{array}$ \\
\hline eventbuilder & $\begin{array}{l}\text { building events (clustering, track building, applying specified } \\
\text { cuts) using data from tbtrack file, setting flags }\end{array}$ \\
\hline flags & $\begin{array}{l}\text { label for specific clusters/hits/tracks to define their properties, like } \\
\text { being good, in a specific region, noisy,... }\end{array}$ \\
\hline central region & $\begin{array}{l}\text { central part of the sensor, excluding the edges; typically the part } \\
\text { with pixels of default size }\end{array}$ \\
\hline translator & $\begin{array}{l}\text { function to manually or automatically (using checkalign values) } \\
\text { apply shifts in x-/y-direction }\end{array}$ \\
\hline cluster masker & $\begin{array}{l}\text { function to mask clusters, containing masked pixels, being in a } \\
\text { bad region,... }\end{array}$ \\
\hline tbmon internal alignment function, based on fit of residual meas- \\
urement
\end{tabular}




\section{A.2 TBmonll}

Available settings in TBmonII main config file:

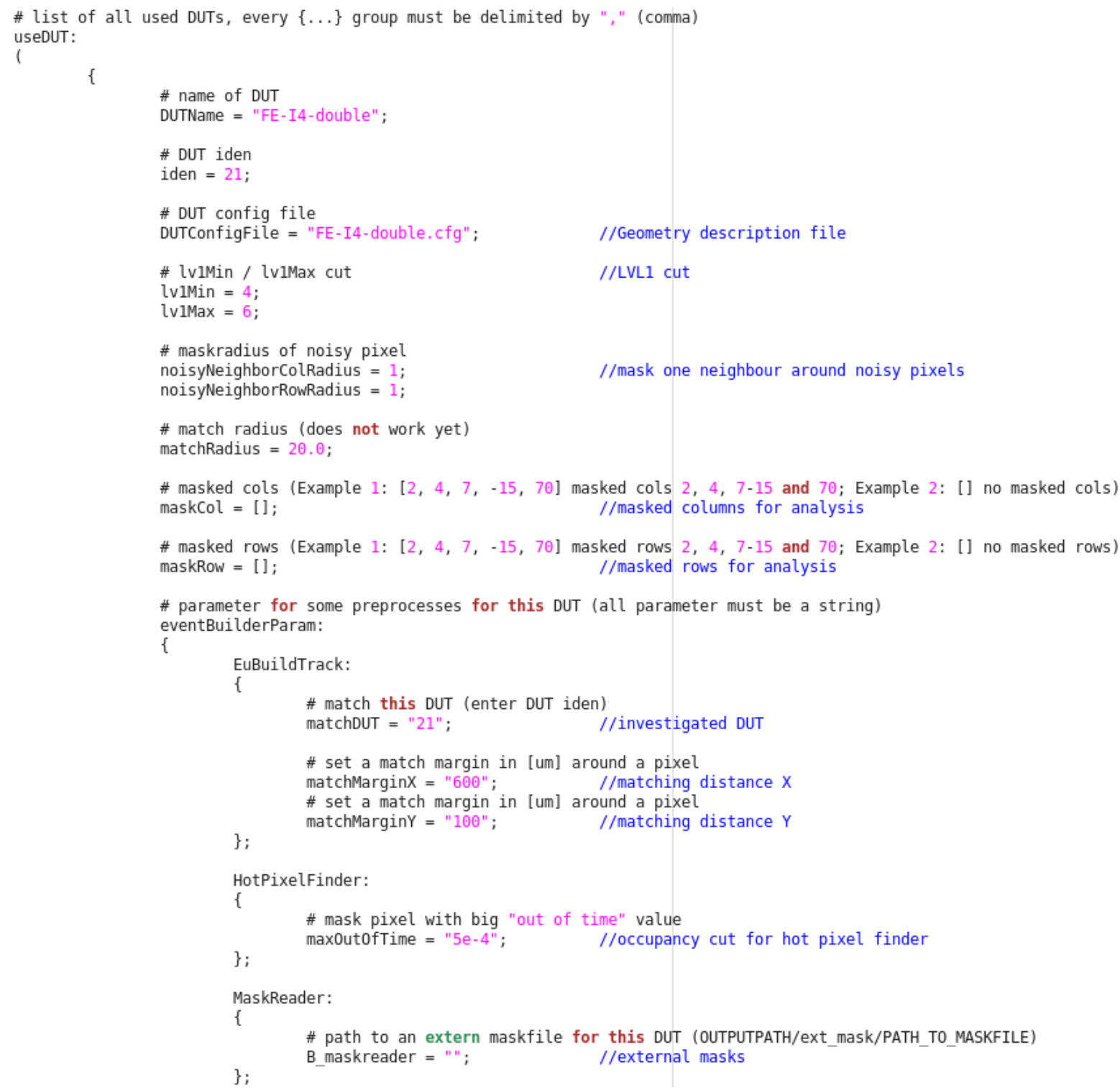

Figure A.1: Section of TBmonII main config, showing the available settings for one DUT. 


\section{Bibliography}

[1] S. Glashow, "Partial Symmetries of Weak Interactions", Nucl. Phys. 22 (1961) 579.

[2] S. Weinberg, “A Model of Leptons”, Phys. Rev. Lett. 19 (1967) 1264.

[3] A. Salam, "Weak and Electronmagnetic Interactions", Nobel Symposium 8 Almqvist \& Wiksell (ed.) (1968).

[4] H. Politzer, "Reliable Perturbative Results for Strong Interactions?", Phys. Rev. Lett. 30 (1973) 1343.

[5] The ATLAS Collaboration, "Observation of a New Particle in the Search for the Standard Model Higgs Boson with the ATLAS Detector at the LHC", Phys.Lett. B 716 (2012) 1-29.

[6] M. Nessi, "Detector Plans for LS1", Proceedings of Chamonix 2012 workshop on LHC Performance (2012), CERN-2012-006 241-243.

[7] L. Evans and P. Bryant, "The CERN Large Hadron Collider: Accelerator and Experiments LHC Machine", JINST 3 (2008) S08001.

[8] E. Holzer et al., "Beam Loss Monitoring for LHC Machine Protection", Physics Procedia 37 (2012) 2055-2062.

[9] J. Nadal, "Simultaneous measurement of the top quark pair production cross-section and $R_{b}$ in the ATLAS experiment", CERN-THESIS-2012-115,

$\mathrm{PhD}$ Thesis, University Autonoma de Barcelona, 2012.

[10] M. Lamont, "Status of the LHC", JPCS 455 (2013) 012001.

[11] The ATLAS Collaboration, "The ATLAS Experiment at the CERN Large Hadron Collider", JINST 3 (2008) S08003.

[12] CMS Collaboration, "The CMS Experiment at the CERN LHC", JINST 3 (2008) S08004.

[13] The LHCb Collaboration, "The LHCb Detector at the LHC", JINST 3 (2008) S08005.

[14] ALICE Collaboration, "The ALICE Experiment at the CERN LHC", JINST 3 (2008) S08002.

[15] The MoEDAL Collaboration, Technical Design Report of the MoEDAL Experiment, CERN-LHC-2009-006, 2010.

[16] TOTEM Collaboration, TOTEM Technical Design Report, CERN-LHCC-2004-002, 2004.

[17] The LHCf Collaboration, "The LHCf detector at the CERN Large Hadron Collider", JINST 3 (2008) S08006.

[18] The ATLAS Collaboration, Physics at a High-Luminosity LHC with ATLAS (Update), ATL-PHYS-PUB-2012-004, 2012.

[19] M. Dolan, C. Englert and M. Spannowsky, "Higgs self-coupling measurements at the LHC", JHEP 10 (2012) 112. 
[20] ATLAS Magnet Project Collaboration, Magnet Project Technical Design Report, Volume 2, Barrel Toroid, CERN/LHCC 97-19, 1997.

[21] H. H. J. ten Kate, "ATLAS Superconducting Toroids and Solenoid", IEEE Trans. Appl. Supercond. 15 (2005) 1267-1270.

[22] ATLAS Magnet Project Collaboration, Magnet Project Technical Design Report, Volume 4, Central Solenoid, CERN/LHCC 97-21, 1997.

[23] L. Mandelli, "The ATLAS Electromagnetic Calorimeters: Features and Performance", Int. J. Mod. Phys. A 25.9 (2010) 1739-1760.

[24] ATLAS Inner Detector Community, Inner Detector Technical Design Report, Volume 1, CERN/LHCC 97-16, 1997.

[25] The ATLAS TRT Collaboration, "The ATLAS Transition Radiation Tracker (TRT) proportional drift tube: design and performance", JINST 3 (2008) P02013.

[26] J. R. Pater, "The ATLAS SemiConductor Tracker operation and performance", JINST 7 (2012) C04001.

[27] W. R. Leo, Techniques for Nuclear and Particle Physics Experiments, Second Revised Edition, Springer-Verlag, 1994.

[28] G. Lutz, Semiconductor Radiaton Detectors, Second Printing, Springer-Verlag, 2001.

[29] J. Beringer et al. (Particle Data Group), "The Review of Particle Physics", Phys. Rev. D86, 010001 (2012 and 2013 partial update for the 2014 edition), Chapter 31, 4.

[30] S. Meroli, D. Passeri and L. Servoli, "Energy loss measurement for charged particles in very thin silicon layers", JINST 6 (2011) P06013.

[31] Z. He, "Review of the Shockley-Ramo theorem and its application in semiconductor gamma-ray detectors", Nucl.Instrum.Meth. A 463 (2001) 250-267.

[32] G. Lynch and O. Dahl, "Approximations to multiple Coulomb scattering", Nucl.Instrum.Meth. B 58 (1991) 6-10.

[33] M. Moll, "Radiation Damage in Silicon Particle Detectors", DESY-THESIS-1999-040, PhD Thesis, University of Hamburg, 1999.

[34] V.A.J. van Lint, T.M. Flanagan, R.E. Leadon, J.A. Naber, V.C. Rogers, Mechanisms of Radiation Effects in Electronic Materials, John Wiley \& Sons, 1980.

[35] F. Hügging, "Der ATLAS-Pixelsensor", UniDo PH-E4-01-05, PhD Thesis, University of Dortmund, 2001.

[36] ROSE Collaboration, 3rd RD48 Status Report, CERN-LHCC-2000-009, 2000.

[37] J. Weingarten, "System Test and Noise Performance Studies at the ATLAS Pixel Detector", BONN-IR-2007-10, PhD Thesis, University of Bonn, 2007.

[38] I. Peric, "The FEI3 readout chip for the ATLAS pixel detector", Nucl.Instrum.Meth. A 565 (2006) 178-187.

[39] T. Wittig, "Slim Edge Studies, Design and Quality Control of Planar ATLAS IBL Pixel Sensors", PhD Thesis, University of Dortmund, 2013. 
[40] J. Große-Knetter, "Vertex Measurement at a Hadron Collider - The ATLAS Pixel Detector", BONN-IR-2008-04, Habilitationsschrift, University of Bonn, 2008.

[41] D. Dobos, "Production accompanying testing of the ATLAS Pixel module", CERN-THESIS-2007-016, Diploma Thesis, University of Dortmund, 2004.

[42] ATLAS IBL Community, ATLAS Insertable B-Layer Technical Design Report, CERN-LHCC-2010-013, 2010.

[43] ATLAS Collaboration, Letter of Intent for the Phase-II Upgrade of the ATLAS Experiment, CERN-LHCC-2012-022 - Draft version for comments, 2012.

[44] ATLAS IBL Collaboration, "Prototype ATLAS IBL Modules using the FE-I4A Front-End Readout Chip", JINST 7 (2012) P11010.

[45] M. Garcia-Sciveres, ATLAS Experiment Pixel Detector Upgrades, Proceedings of Meeting of the Division of Particles and Fields of the American Physical Society, DFF 2011, 2011, arXiv: 1109.4662v1 [physics.ins-det].

[46] ATLAS IBL Community, ATLAS Insertable B-Layer Technical Design Report Addendum, CERN-LHCC-2012-009, 2012.

[47] S. Parker, C. Kenney and J. Segal, "3D - A proposed new architecture for solid-state radiation detectors", Nucl.Instrum.Meth. A 395 (1997) 328-343.

[48] C. Da Via, 3D active edge silicon sensors - Presentation at the Vertex 2006 Conference, http://indico.cern.ch/event/1151/session/11/contribution/35/material/slides/0.pdf, 2006.

[49] C. Da Via et al., "3D active edge silicon sensors with different electrode configurations: Radiation hardness and noise performance", Nucl.Instrum.Meth. A 604 (2009) 505-511.

[50] M. Povoli et al., "Slim edges in double-sided silicon 3D detectors", JINST 7 (2012) C01015.

[51] I. Mandic et al., "Observation of Full Charge Collection Efficiency in Heavily Irradiated n+p

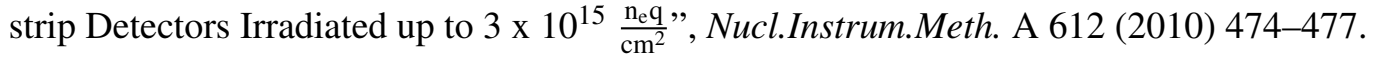

[52] A. Affolder, P. Allport and G. Casse, "Charge Collection Efficiency Measurements of Heavily Irradiated Segmented n-in-p and p-in-n Silicon Detectors for Use at the Super-LHC", IEEE Tr.Nucl.Sci. 56 (2009) 765-770.

[53] W. Trischuk et al., “The ATLAS Beam Conditions Monitor”, JINST 3 (2008) P02004.

[54] H. Kagan, M. Mikuz and W. Trischuk, ATLAS Diamond Beam Monitor (DBM) - Project Document, Rev. 2, ATL-IP-ES-0187, 2012.

[55] D. Ferrere, IBL Project Overview - Stave coating/potting review Presentation, 31.01.2014.

[56] I. Rubinskiy, "An EUDET/AIDA pixel beam telescope for detector development", Physics Procedia 37 (2012) 923-931.

[57] J. Behr, Test Beam Measurements with the EUDET Pixel Telescope, EUDET-Report-2010-01, 2010.

[58] A. Dorokhov, Optimization of amplifiers for monolithic active pixel sensors, Proceedings of Topical Workshop on Electronics for Particle Physics 2007 (TWEPP 07), 2007 423-427. 
[59] M. Deveaux et al.,

"Radiation Tolerance of CMOS Monolithic Active Pixel Sensors with Self-Biased Pixels" (2009), arXiv: 0908.4202v1 [physics .ins-det].

[60] D. Cussans, Description of the JRA1 Trigger Logic Unit (TLU), v0.2c, EUDET-Memo-2009-04, 2009.

[61] M. Backhaus et al.,

"Development of a versatile and modular test system for ATLAS hybrid pixel detectors", Nucl.Instrum.Meth. A 650 (2011) 37-40.

[62] University of Bonn, USBpix - USB based readout system for ATLAS FE-I3 and FE-I4, TWiki, URL: http://icwiki . physik . uni-bonn. de/twiki/bin/view/Systems/UsbPix (visited on $04 / 03 / 2014)$.

[63] F. Schuster, "Development of New Methods of Data Transmission for the Upgraded ATLAS Pixel Detector at the HL-LHC", II.Physik-UniGö-MSc-2013/01,

Master Thesis, University of Göttingen, 2013.

[64] E. Corrin, EUDAQ Software User Manual, EUDET-Memo-2010-01, 2010.

[65] D. Autiero et al., Characterization of the T24 electron beam line available at DESY, internal report, 2004.

[66] T. Behnke et al., Test Beams at DESY, EUDET-Memo-2007-11, 2007.

[67] F. Gaede, "Marlin and LCCD - Software tools for the ILC", Nucl.Instrum.Meth. A 559 (2006) 177-180.

[68] A. Bulgheroni, EUTelescope, the JRA1 tracking and reconstruction software: a status report, EUDET-Memo-2008-48, 2008.

[69] V. Blobel and C. Kleinwort, "A New method for the high precision alignment of track detectors" (2002), arXiv: hep-ex/0208021 [hep-ex].

[70] R. Frühwirt and A. Strandlie, "Track fitting with ambiguities and noise: A study of elastic tracking and nonlinear filters", Comput. Phys. Commun. 120 (1999) 197-214.

[71] R. Turchetta, "Spatial Resolution of Silicon Microstrip Detectors", Nucl.Instrum.Meth. A 335 (1993) 44-48.

[72] R. Nagai et al., "Evaluation of novel KEK/HPK n-in-p pixel sensors for ATLAS upgrade with testbeam", Nucl.Instrum.Meth. A 699 (2013) 78-83.

[73] J. Weingarten et al., "Planar pixel sensors for the ATLAS upgrade: beam tests results", JINST 7 (2012) P10028.

[74] M. Morpurgo,

"A large superconducting dipole cooled by forced circulation of two phase helium", Cryogenics 01 (1979) 411-414.

[75] FE-I4 Collaboration, The FE-I4B Integrated Circuit Guide, Version 1.7, 2011.

[76] M. George, "Recent Achievements of the ATLAS Upgrade Planar Pixel Sensors R\&D Project", JINST 9 (2014) C05004. 
[77] B. Lemmer, Bis(s) ins Innere des Protons : ein Science Slam durch die Welt der Elementarteilchen, der Beschleuniger und Supernerds, Berlin, Heidelberg: Springer Spektrum, 2014.

[78] J. Weingarten, private communication. 



\section{List of Figures}

2.1 Schematic of the CERN accelerator system and the positions of the four LHC experiments (figure redrawn by [9]). . . . . . . . . . . . . . . . . . 4

2.2 Cross section of a cryo-dipole at the Large Hadron Collider [7]. . . . . . . . . . . . . . 5

2.3 Left: Simulated invariant mass distribution in the $t \bar{t} H, H \longrightarrow \gamma \gamma$ channel for the estimated full HL-LHC luminosity of $3000 \mathrm{fb}^{-1}$. Right: Simulated invariant mass distribution for the inclusive $H \longrightarrow \mu \mu$ channel, again for a total luminosity of $3000 \mathrm{fb}^{-1}$. In red the expected signal is shown, the other colours describe the background [18]. . . . . . . .

2.4 Some example Feynman diagrams, which contribute to $p p \longrightarrow h h+X$. While graphs a) and b) contribute to the background in a Higgs self coupling measurement, graph c) is the process of interest $[19] \ldots \ldots \ldots \ldots \ldots \ldots$

2.5 Feynman diagram for the $H H \longrightarrow b \bar{b} \gamma \gamma$ channel, which should be investigated at the HL-LHC. . . . . . . . . . . . . . . . . . . .

2.6 Exemplary Feynman diagram for a vector boson scattering process, which should be investigated at the HL-LHC. . . . . . . . . . . . . . . . . . . .

2.7 Schematic layout of the ATLAS Detector, indicating its dimensions and the different subsystems. A description of the detector systems can be found in section 2.3.1 and [11]. 9

2.8 Schematic overview for the different parts of the ATLAS muon system [11] . . . . . 10

2.9 The ATLAS barrel toroid, shown in a schematic sketch. Beside the eight barrel coils, the two end cap toroids are shown, where the one on the right side is inserted to its final position and the left one extracted $[21] \ldots \ldots \ldots \ldots$

2.10 A schematic view of an ATLAS end cap toroid, showing the eight coils, which are kept in a vacuum vessel $[21] . \ldots \ldots \ldots \ldots \ldots$

2.11 Schematic cut of the ATLAS calorimeter system, showing the different components of the hadronic and the electromagnetic calorimeter [11] . . . . . . . . . . .

2.12 Structure of an ATLAS hadronic calorimeter cutaway view, showing the tile layout of steel absorbers and scintillators. Beside that, parts of the optical components are shown

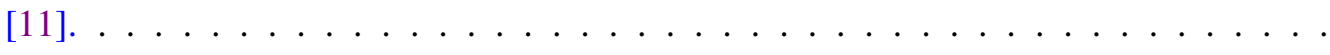

2.13 Sketch of the ATLAS hadronic forward calorimeter, showing the metal matrix structure and a zoom into the setting of the high voltage rods, which are surrounded by the active medium, the liquid argon $[11] \ldots \ldots \ldots \ldots$

2.14 Left: Sketch of the electromagnetic barrel calorimeter at $\eta=0$. The accordion structure and the segmentation of the calorimeter is shown [23]. Right: Photograph of an electromagnetic barrel calorimeter module, which is partly stacked, and the calorimeter support rings in the back $[11] \ldots \ldots \ldots \ldots \ldots$

2.15 Sketch of the drift model of ionized electrons in an ATLAS transition radiation tracker

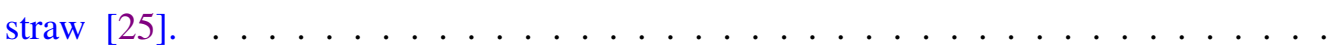

2.16 Photograph of an ATLAS SemiConductor Tracker end-cap module, indicating the different components of the module $[26] \ldots \ldots \ldots \ldots \ldots$ 
3.1 Average stopping power of muons in copper for different ranges of $\beta \gamma$. The light blue vertical bands indicate the limits of different models, used as approximations [29]. . . .

3.2 Energy loss for $12 \mathrm{GeV}$ protons in silicon absorbers of different thicknesses. Landau functions are fitted to the different distributions [30]. . . . . . . . . . . . . . .

3.3 Left: Photons with energies equal, larger and smaller than the band gap are absorbed and lift electrons to an intermediate state or the conduction band. Simultaneously holes are created in the valence band. Right: Energy loss of a charged pion in silicon due to ionization versus the kinetic energy of the pion. The dotted line shows the progression including shell and density corrections $[28] \ldots \ldots \ldots \ldots$

3.4 Left: cross section vs photon energy for the photoelectric effect (in lead), centre: cross section vs photon energy for Compton scattering and right: cross section vs photon energy for pair production [27]. . . . . . . . . . . . . . . .

3.5 Schematic drawing of the energy band structure for semiconductors, with a band gap on the order or few eV [28] . . . . . . . . . . . . . . . . . . .

3.6 Examples for doped silicon: n-type semiconductor with an excess of electrons (left), p-type semiconductor with an additional holes (right) [28] . . . . . . . . . . . .

3.7 The ATLAS pixel detector system, showing the three cylindrical barrel layers, as well as three end-cap discs per side (ATLAS Experiment (c) 2013 CERN). . . . . . . . . . .

3.8 Top: Schematic drawing of a barrel ATLAS pixel module, illustrating the hybrid module concept. Shown are the module flex, including electronics to handle the different FrontEnd chips, the sensor itself and the 16 Front-End chips. Bottom: Photograph of a full pixel module $[11] \ldots \ldots \ldots \ldots \ldots \ldots$

3.9 Comparison of the effective doping concentration/depletion voltage as a function of $1 \mathrm{MeV}$ neutron equivalent fluence for standard and oxygen enriched float zone silicon. Especially for fluences above the type inversion point, the advantages in terms of depletion voltage for oxygenated silicon are clearly visible $[36] . \ldots \ldots \ldots$

3.10 Schematic of the sensor layout in the region between four readout chips. Ganged pixels are used to cover the inter-chip region [37]. . . . . . . . . . . . .

3.11 Schematic drawing of the depletion region before and after irradiation for $n$-in-n sensors in underdepleted operation $[35] . \ldots \ldots \ldots \ldots$

3.12 Simplified schematic of the analogue part of the FE-I3 chip, including a bump bond, test circuits and the preamplifier and discriminator chain, forming the read out signal [39]. .

3.13 Dependencies on the injected charge, the threshold and the feedback current of the time over threshold (ToT) value in the FE chip preamplifier and discriminator [41] . . . . . .

4.1 Technical drawing of the IBL layout in $\mathrm{R} \phi$ view, showing the new beampipe, three staves and the IBL envelopes [42]. . . . . . . . . . . . . . . . . . .

4.2 Rendered view in z-direction of the ATLAS Insertable b-Layer inside the existing inner pixel system layer, showing the arrangement of support structures and sensors. The picture especially shows that the IBL arrangement allows a small sensor overlap in $\phi$ direction, but not in z-direction due to the limited space [42] . . . . . . . . . . .

4.3 FE-I4A chip, as used for IBL module testing and to scale FE-I3 chip, used in the current pixel detector. In comparison, the new chip grew significantly in size and number of pixel cells, while being produced in a more radiation tolerant process [44] . . . . . . .

4.4 Sketch of the pixel logic and peripheral structures in the bottom $2 \mathrm{~mm}$ of a FE-I4A chip [44]. 
4.5 Baseline layout for the new all silicon tracker after the phase 2 upgrade. In red four barrel layers and six end-caps per side are shown for the pixel system, in blue the new strip detector [43] . . . . . . . . . . . . . . . . . . . .

4.6 Left: IBL double chip module format for planar silicon sensors. Each sensor has the size of two FE-I4 chips and is arranged with extended pixels in the inter-chip region, to have a sensitive area in the central part. Right: IBL single chip module format for 3D silicon sensors. The sensor size corresponds to the size of a FE-I4 chip [42] . . . . . .

4.7 Left: schematic of a particle crossing a 3D silicon sensor. The created charge carriers drift in the horizontal direction, with a maximum drift distance of several $10 \mu \mathrm{m}$. Right: the same situation for a planar sensor, where the charge carriers drift a maximum distance of few $100 \mu \mathrm{m}$. In both cases the drift values naturally depend on the exact design [48] . . . . . . . . . . . . . . . . . . . . .

4.8 Simulated most probable value for the collected charge, using a 3D sensor with the IBL candidate specifications, which are a thickness of $230 \mu \mathrm{m}$ and a 2E-250 electrode configuration [42].

4.9 Left: 3D sensor design, based on a double sided production, where the columns do not reach through the whole sensor thickness. A fraction $d$ (around $20 \mu \mathrm{m}$ ) of the sensor thickness is not equipped with columns. Right: full 3D sensors. The columns of both doping types reach through the full sensor thickness $[42] \ldots \ldots \ldots \ldots$

4.10 Two different planar n-in-n silicon design candidates, as they were investigated for IBL. Figure a) is the edge region schematic of a "conservative" design, where the number of guard rings is reduced from 16 (current ATLAS pixel sensor) to 13 and the edge width reduced to $450 \mu \mathrm{m}$. Part b) shows the edge region of a "slim edge" design. Most of the guard rings are overlapping with the outermost pixels, which allows to further reduce the inactive part. Since the total dimensions of a sensor are fixed, the outermost pixels are extended to a length of $500 \mu \mathrm{m}$. The extra length covers the region which is inactive in the conservative design $[42] \ldots \ldots \ldots \ldots \ldots$

4.11 IBL candidate planar n-in-p silicon design with an inactive edge width of $450 \mu \mathrm{m}$ [42].

4.12 Picture of the extracted pixel detector including support structure and services, indicat-

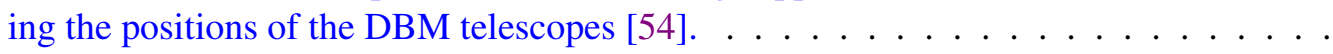

4.13 Left: CAD drawing of the DBM setup on one side of the interaction point, showing the four telescope arms and the supply cables. Right: DBM cooling structure (in blue) and the connection to the $\mathrm{nSQP}$, on which the Diamond Beam Monitor is mounted [46]. . .

4.14 Dressed IBL modules [55] . . . . . . . . . . . . . . . . . .

5.1 The EUDET telescope, set up at DESY for measurements of the ATLAS pixel group. On the left, as well as on the other side of the telescope, two crossed scintillators are mounted for triggering. The two telescope arms, each equipped with three telescope planes, can be seen, as well as the telescope cooling. In the centre the baseplate of the ATLAS pixel cooling box is mounted, with two devices under test on it. For further details, see 5.1 and $5.1 .1 \ldots \ldots \ldots \ldots \ldots$

5.2 Schematic of a Monolithic Active Pixel Sensor (MAPS) in cross section view. The induced charge carriers produced by the incident particle diffuse towards the n-well, where the charge is collected $[57] \ldots \ldots \ldots \ldots \ldots$ 
5.3 Typical IBL testbeam setup, having one telescope arm upstream and one telescope arm downstream. Between the telescope arms, the DUT cooling box is visible, which allows mounting of several DUTs inside, as well as space for dry ice. The cooling is necessary to keep the leakage current of the devices low, to avoid damages. Since the operation parameters of the IBL experiment specify a maximum operation temperature of $-15^{\circ} \mathrm{C}$, the dry ice cooling is a legitimate way to keep the devices within their design conditions.

5.4 Left: A trigger logic unit (TLU) as it has been used in the ATLAS pixel testbeams. Up to six devices under test can be connected. Right: Data lines of a TLU, showing a trigger handshake, where data is sent from the TLU to the connected devices to synchronize the setup [60] . . . . . . . . . . . . . . . . . . . . .

5.5 USBpix test system, consisting of a Multi-IO board, equipped with an FPGA and a micro controller, and an adapter card. Shown in this picture is a setup for FE-I4 devices [62]. 50

5.6 RCE test setup, as it is used for further developments of the RCE system. The same RCE setup was also used for device readout in the latest testbeam campaigns. The RCE processors are located below the black heat sinks. . . . . . . . . . . . . .

5.7 Schematic of a complete RCE setup, including optical data transmission and the usage of several RCE boards [63] . . . . . . . . . . . . . . . . . . . . . . .

5.8 Schematic overview of the EUDAQ structure, grouped into the main producers. All producers are connected via Ethernet. For further details, see 5.1.4 [64]. . . . . . . . .

5.9 Online Monitor screenshot, showing the number of clusters for the second and third telescope plane during data taking. These plots give a first live impression of the data quality.

5.10 Hit correlations between all devices in the used setup with respect to the first telescope plane. The Online Monitor correlation plots do not take into account any information that is provided in the geometry description file, but still they can give a good estimate for the alignment of the setup. Note: the Online Monitor screenshots were taken during a testbeam campaign of the planar pixel sensors community in November 2013 . . . . .

5.11 Hitmaps for the two devices under test. On the left side the position in coordinates of $\mathrm{X} / \mathrm{Y}$ in units of pixels are shown for a 4-chip module, on the right side the same information is provided for a single chip module. In both cases the crossing area of the trigger scintillators is clearly visible. . . . . . . . . . . . .

5.12 Screenshot of all information the Online Monitor provides for a device under test. The considered device is a single chip module. Since the histograms are being filled during data taking and the necessary calculations for the different kinds of histograms need different computing power, not all histograms are filled in the same speed. . . . . . . .

5.13 Schematic layout of the electron/positron production at DESY for testbeam users. The shown dipole magnet is used for spreading the particle beam into a horizontal fan, such that particles are separated in terms of charge sign and energy [65] . . . . . . . . .

5.14 Particle rates at DESY testbeam, depending on the chosen energy and the secondary beam target. The particle energy in the beamline can be varied by the users between $1 \mathrm{GeV}$ and $6 \mathrm{GeV}[66] \ldots \ldots \ldots \ldots \ldots$

5.15 Schematic overview of the CERN North Area beamlines, including the primary targets and wobbling stations, which allow users to choose between a variety of particles and particle energies (taken from CERN NA62 Collaboration website; at time of writing http://na62.web.cern.ch/na62/ExperimentalArea/SchematicNorthArea.html). . . . . . . 
6.1 Track reconstruction chain, as used for ATLAS pixel testbeams. The raw data is read in and after five processing steps, a ROOT file containing all relevant track and hit information for further analysis is generated. . . . . . . . . . . . .

6.2 Example of possible coordinate system rotations in the GEAR library, using rotation

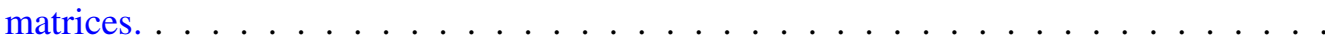

6.3 With each trigger signal, the trigger IDs written into the data streams are increased by 1. In case of hardware incompatibilities or software problems, different TLU trigger IDs could be written into the data streams of the telescope and the ATLAS pixel DUT, resulting in desynchronized data streams. . . . . . . . . . .

6.4 The sparse clustering algorithm performs a search for neighbouring hits, starting from the seed pixel. In a first step cells 1 to 3 are checked, afterwards 4 and 5 and finally cells 6 to 8 . More details on the functionality can be found in the text. . . . . . . . .

6.5 Example of a 4-hit cluster and a 2-hit cluster in one event. Each hit in the event gets assigned a number for further processing in the cluster finding algorithm. . . . . . . .

6.6 Left: the digital center of gravity algorithm sets the cluster center to the geometrical center of the cluster. Right: the analogue center of gravity weights the position calculation with the ToT value. Thus, the cluster position is shifted towards the pixel with higher charge. . . . . . . . . . . . . . . . . . .

6.7 Left: Clustersize distribution for a non-rotated APIX sensor ( $\mathrm{x}$ - and $\mathrm{y}$-direction multiplied). The average clustersize is around 1.2, as expected. Right: Event multiplicity of the first telescope plane in the setup. Due to the long integration time, on average around 4.8 clusters are found on this plane per event. . . . . . . . . . . .

6.8 Cluster ToT spectrum of an APIX device. The chip has been tuned to a response of 6 ToT units for the expected standard signal of a passing particle. The distribution shows a peak around 6 ToT, with the expected Landau tail. . . . . . . . . . . . . . .

6.9 Control plot: Hit position correlations in x-direction of the hits on the first and the second telescope plane. The expected rising correlation band is clearly visible (green), as well as the combinatorial background (blue). The shape of the correlations shows that the rotations set in the GEAR file are correct and the correlation band offset is small. Thus, only small corrections will be made by the pre-aligner. . . . . . . . . . . . .

6.10 Left: residual distribution of an APIX device before alignment. The distribution is shifted from 0 and includes some outliers. Right: residual distribution of the same device after alignment. Now the mean of the distribution is close to 0 and the width represents the spatial resolution of the APIX device in $\mathrm{x}$-direction. . . . . . . . . .

6.11 One of the background suppression parameters in the alignment step is DistanceMax. This value defines the opening of a cone, within which tracks, extrapolated to the subsequent plane, are being searched for. This value is specified in $\mu \mathrm{m}$ per $10 \mathrm{~cm}$ z-distance between two planes. . . . . . . . . . . . . . . . . .

6.12 General structure of TBmon: track files are read in, a preprocessing is performed on the dataset, followed by the analyses and finally the results are written into a ROOT file. .

6.13 Schematic structure of TBmon. Several tbtrack-files, hosting a certain number of events, can be combined for processing, if they were taken under the same conditions. Different preprocessing sequences are performed on the data and cuts can be applied, followed by the analysis processors. The results can be written into single graphics files (e.g. jpg format) or collected in a ROOT file. . . . . . . . . . . . . . . . . 
6.14 Schematic drawing of the TBmon looper functionality, which is the central part of the track builder. The looper reads information for all DUTs, except the investigated one, to check for in-time hits in the investigated DUT and match them to the respective track.

6.15 Left: expected LVL1 distribution of a tuned ATLAS pixel device. The hits are registered after a certain latency tuned to 6 clock cycles ( 25 ns per LVL1), plus and minus one LVL1 bin, due to effects like timewalk. Right: LVL1 distribution of a device dominated by noise hits. A uniformly distributed noise floor is visible, having a peak around the tuned latency value (note: here the y-axis starts at 85000 entries). . . . . . . . . . . .

6.16 Exemplary histogram output of the TBmon internal CheckAlign processor. After applying several quality cuts, the averaged residual is plotted against the hit position for the four possible combinations: residual $\mathrm{X}$ vs hit $\mathrm{X}$, residual $\mathrm{Y}$ vs hit $\mathrm{X}$, residual $\mathrm{X}$ vs hit $\mathrm{Y}$ and residual $\mathrm{Y}$ vs hit $\mathrm{Y}$. The data points far off the horizontal lines are at the edge of the scintillator region, thus they have low statistics and large errors. . . . . . . . . . .

6.17 Exemplary residual distributions in $\mathrm{x}$ - and y-direction for a FE-I4 chip based device. A box fit with and without slope in the plateau region is used to determine the width of the distribution, the mean value and the RMS. Note: the structure visible in the plateau regions of the shown examples can be explained due to the different residual distributions for different cluster sizes and the coarse charge resolution of the FE-I4 chip, which has an influence on the calculated charge weighted positions. In addition, the not perfect alignment of the devices in this exemplary dataset influences the flatness of the distribution. . . . . . . . . . . . . . . . . . . . . .

6.18 Typical $\chi^{2}$ distribution of the tracks, used for the analysis of a FE-I4 based device. This distribution shows the whole $\chi^{2}$ spectrum, to give the user a cross-check for the applied

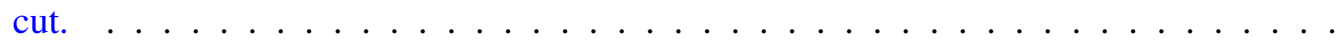

6.19 Track map created by the BeamProfile processor, showing all tracks going through the central region of the device that pass the $\chi^{2}$ cut. This track map gives an impression of the beam spot size and an indirect impression of the scattering magnitude. Here the track map shows that the particle beam was focussed a bit towards the upper left of the

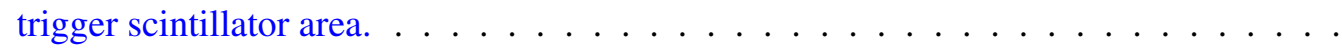

6.20 The histogram shows the number of hits per event. It can be seen, that events can occur, having no hit at all because of a track that missed the device, but the expected peak for

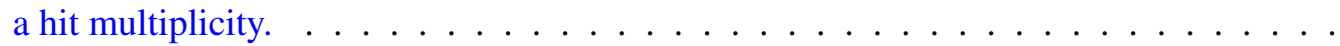

6.21 Left: cluster sizes detected in the investigated device, taking into account only clusters that can be matched to a track, where the same track has to be matched also in a reference device. Right: cluster size distribution of clusters without a matched track. The relatively high number of un-matched clusters can be explained by the fact that TBmon only treats one track per event. . . . . . . . . . . . . . .

6.22 Exemplary histogram showing the cluster multiplicity of a device. As for the hit multiplicity, a value of 0 is caused by events in which a track was matched to other devices in the setup, but either missed the investigated device or was not detected. . . . . . . .

6.23 Left: Hitmap of a device without applying any cuts, just counting the number of hits per pixel cell. Right: Hitmap of the same device after applying timing cuts and only taking into account hits that can be matched to a track. Note that all hits outside of the projected trigger scintillator area vanish. . . . . . . . . . . . . . 
6.24 Track map of the investigated device, which displays the incident positions of tracks that were matched in a reference plane and in the investigated device. This example of a track map shows the effect of having a device with a large number of noisy pixels, which are visible as white spots in the histogram. . . . . . . . . . . . . . .

6.25 The left histogram shows a typical example for a device with a significant number of noisy (masked) pixels and a slight general efficiency drop in the first columns. In the right histogram the efficiency of a device is shown, which was used as reference plane. Only a few pixels were masked and the efficiency homogeneous and high over the device. 83

6.26 Charge collection map for a device with a pixel pitch of $250 \mu \mathrm{m} \times 50 \mu \mathrm{m}$. The average collected charge in units of ToT against the position within a pixel cell is projected into a histogram of a single pixel size. On the right side of the histogram a typical decrease in the collected charge due to the bias dot is visible. Because of the low beam energy used for this measurement (DESY testbeam), the shape of the bias dot is not clearly visible in the histogram. . . . . . . . . . . . . . .

6.27 The average collected charge of the investigated device is projected on the long side of one pixel with the standard size $250 \mu \mathrm{m} \times 50 \mu \mathrm{m}$. In the left histogram a typical ToT profile of a planar silicon device is shown, the right histogram shows an exemplary histogram of a 3D silicon device. . . . . . . . . . . . . .

6.28 Three typical examples for charge sharing maps, where the charge sharing probability depending on the track incidence point is folded into one pixel and half of the neighbouring pixel in each direction. In the center of each histogram, a standard $250 \mu \mathrm{m} \times 50 \mu \mathrm{m}$ pixel cell is indicated by the black frame. . . . . . . . . . . . .

6.29 Typical output of the SumToT processor, showing on the left the highest charge value of each matched cluster in units of ToT, including the ToT overflow bin at value 14. On the right the charge in units of ToT is histogrammed summed for matched clusters. The device was tuned to a response of 7 ToT units for an injected charge of 20,000 $\mathrm{e}^{-} \ldots$.

6.30 TBmonII Logo (designed by Alexander Granget). . . . . . . . . . . . . . . . .

6.31 Schematic drawing of a typical FE-I4 chip based sensor design, as it is modelled in TBmon and TBmonII. The green rectangles represent the edge pixels, the blue rectangles the regular pixels with a dimension of $250 \mu \mathrm{m} \times 50 \mu \mathrm{m} . \ldots \ldots \ldots$

6.32 Schematic drawing of a FE-I4 double chip sensor, as it is modelled in TBmonII. Again the green rectangles model the edge pixels and the blue rectangles the central pixels. On top of that the central double column (orange), having pixels with a pitch of $450 \mu \mathrm{m} \times$ $50 \mu \mathrm{m}$, can be included correctly. . . . . . . . . . . . .

6.33 Schematic drawing of a FE-I4 four chip sensor, as it can be described in TBmonII. The colour code is the same as in figure 6.32, but additionally an inactive region can be included in the description. . . . . . . . . . . . . . . .

6.34 Staggered pixel sensor designs, as the one in this schematic, can be described in TBmonII. The geometry description allows different initial coordinates for each geometry, which makes a proper description of the staggered design possible. . . . . . . . . .

6.35 As shown in the drawing, TBmonII offers the possibility to model pixels with nonrectangular shapes. Here L-shaped pixels are modelled by combining two rectangular substructures to one L-shaped pixel type. Each pixel type can be described by a combination of several of these substructures. . . . . . . . . . . . . 
7.1 Sensor edge designs for different planar pixel sensors. Figure a) shows the sensor edge design of the current ATLAS pixel sensors, with a total edge width of $1100 \mu \mathrm{m}$. Figure b) shows the conservative IBL sensor design with a reduced edge width of $450 \mu \mathrm{m}$, due to less guard rings and smaller safety margins. Finally, figure c) shows the slim edge design, with guard rings shifted on top of the edge pixel, resulting in an edge width of $200 \mu \mathrm{m} \mathrm{[39].} \mathrm{\ldots} \mathrm{.} \mathrm{.} \mathrm{.} \mathrm{.} \mathrm{.} \mathrm{.} \mathrm{.} \mathrm{.} \mathrm{.} \mathrm{.} \mathrm{.} \mathrm{.} \mathrm{.} \mathrm{.} \mathrm{.} \mathrm{.} \mathrm{.} \mathrm{.} \mathrm{.} \mathrm{.}$

7.2 Average hit efficiency of device SCC41, which is an unirradiated planar conservative design. Already at moderate bias voltages of $60 \mathrm{~V}$, the sensor fulfils the IBL requirement. Increasing bias voltages or measurements in magnetic fields, increase the hit efficiency even further. Reminder: the calculated statistical error is in all investigated cases less than $\pm 0.06 \% \ldots \ldots \ldots \ldots \ldots \ldots$

7.3 In-pixel efficiency for device SCC41 (planar, unirradiated, conservative design) at perpendicular incidence, folded into one pixel and half of its neighbours. The black rectangle in the centre of the histogram frames a standard $250 \times 50 \mu \mathrm{m}^{2}$ pixel. At the bottom, a sketch of the planar pixel cell structure is shown (taken from [44]). . . . . .

7.4 Hit efficiency for the edge columns of device SCC41. The histograms show the efficiency in the centre of the histograms, where the vertical black lines indicate a pixel width of $250 \mu \mathrm{m}$ and the red lines mark the position of the edge pixel after alignment. For all edge pixel histograms, the results of the first and last column edge pixels are folded into one histogram. Top: edge pixel efficiency at a bias voltage of $100 \mathrm{~V}, 3200 \mathrm{e}^{-}$ threshold and perpendicular incidence. Bottom: edge pixel efficiency at the same settings in magnetic field. . . . . . . . . . . . . . . . .

7.5 Left: ToT distribution of device SCC41 in the measurement without magnetic field. Right: the same measurement in the $1.54 \mathrm{~T}$ field of the Morpurgo magnet. The distribution of the measurement in the magnetic field is slightly shifted towards higher values, which corresponds to the longer particle path through the sensor and the larger cluster sizes. As fit function a convolution of a Gauss and a Landau distribution is used. . . . .

7.6 Left: structure of DO3 sensor, with blocks of ten edge pixels shifted in steps of $25 \mu \mathrm{m}$ towards the sensor edge. To avoid effects of neighbouring sets in the analysis results, only the innermost eight pixels of each set are used (black frame). Right: collected charge in shifted edge pixels of DO3 at a bias voltage of $100 \mathrm{~V}$. Data of pixels from the same shift-block are folded into the size of one pixel [73] . . . . . . . . . . . . . .

7.7 Average hit efficiency of $250 \mu \mathrm{m}$ thick planar slim edge sensors. Device SCC31 is an unirradiated reference sensor, while LUB2 is irradiated to $4 \cdot 10^{15} \frac{\mathrm{n}_{\mathrm{eq}}}{\mathrm{cm}^{2}}$. The unirradiated sensor already shows a high efficiency of more than $99.5 \%$ for low bias voltages, the irradiated sensor reaches its plateau efficiency at a bias above $800 \mathrm{~V}$. . . . . . . . . . .

7.8 Left: ToT distribution of device LUB2 at a bias voltage of $1000 \mathrm{~V}$. Right: the same measurement at a bias voltage of $600 \mathrm{~V}$. The collected charge for the lower voltage measurement is significantly decreased, which is consistent with the lower hit efficiency.

7.9 Edge efficiency of LUB2 device for bias voltages of $1000 \mathrm{~V}, 800 \mathrm{~V}, 600 \mathrm{~V}, 400 \mathrm{~V}$ and finally ramped to $1000 \mathrm{~V}$ again. . . . . . . . . . . . . . . . .

7.10 Average hit efficienciy of $200 \mu \mathrm{m}$ thick planar slim edge sensors, measured for different voltages, thresholds and in magnetic field. The efficiency fulfils the IBL requirements under all tested conditions. . . . . . . . . . . . . . . . . 
7.11 In-pixel efficiency for devices SCC40 (planar, slim edge, unirradiated) and LUB4 (planar, slim edge, irradiated) at perpendicular incidence, folded into one pixel and half of its neighbours. For comparison, the pixel cell structure is printed below. The drop in efficiency for irradiated planar devices is strongest at the bias rail and bias dot structure.

7.12 Average hit efficiencies of $200 \mu \mathrm{m}$ thick planar slim edge sensors after irradiation, measured for different voltages and thresholds. . . . . . . . . . . . . . .

7.13 Histogram a) shows the detected cluster charge for device PS2 at a bias voltage of $1000 \mathrm{~V}$ and a threshold of $1500 \mathrm{e}^{-}$in units of ToT. In histogram b) the same measurement again at $1000 \mathrm{~V}$ bias voltage is shown, but for a signal threshold of $1800 \mathrm{e}^{-}$. Histogram c) shows the cluster ToT measurement again for device PS2 at a bias voltage of $600 \mathrm{~V}$ and at a threshold of $1800 \mathrm{e}^{-}$. In histogram d) the measurement was repeated for a bias voltage of $600 \mathrm{~V}$ and again a threshold of $1800 \mathrm{e}^{-} \ldots \ldots \ldots$. . . . . . .

7.14 Comparison of the LVL1 distribution for device PS2 at $1000 \mathrm{~V}$ on the left and $600 \mathrm{~V}$ on the right. For the higher bias voltage the noise floor is at $6 \%$ of the peak height, for the lower bias voltage the noise floor is at $15 \%$ of the peak height. The green area shows the default acceptance window of LVL1 values between $[0,10] \ldots \ldots$. . . . . . . . .

7.15 Comparison of the efficiency maps for device PS2 (left) and SCC61 (right). Since a significant fraction of pixels had to be masked for SCC61 and the analysis automatically masks the neighbour pixels of each masked pixel, the efficiency calculation of this device is not representative. Note: the missing region in the efficiency map of PS2 is caused by the reference device FBK13, which had a bump-bonding issue in this region. Since FBK13 was the reference device, no tracks could be matched in that region. . . .

7.16 Average hit efficiencies of unirradiated double sided 3D devices for different threshold settings, bias voltages and incident angles. . . . . . . . . . . .

7.17 In-pixel efficiency for devices SCC55 (3D, double sided, unirradiated) and SCC34 (3D, double sided, unirradiated) at perpendicular incidence, folded into one pixel and half of its neighbours. For comparison, the pixel cell structure is printed below. . . . . . . . .

7.18 Average hit efficiencies of irradiated double sided 3D devices. The large drop in efficiency even at non-perpendicular incidence of device SCC82 compared to the other devices indicates, that the collected charge of the device is around the threshold value

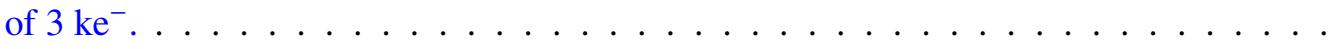

7.19 Edge efficiencies of devices SCC34 and SCC97 at a bias voltage of $140 \mathrm{~V}$. The plateau efficiency values and the visible structures in the efficiency distribution indicate, that the devices were not fully depleted. Even in underdepleted operation the edge efficiency level is uniform over the whole edge pixel width. . . . . . . . . . . .

7.20 ToT distribution of device SCC82 at a bias voltage of $160 \mathrm{~V}$, signal threshold of $3200 \mathrm{e}^{-}$ and a feedback tuning to $8 \mathrm{ToT}$ at $20 \mathrm{ke}^{-}$. The distribution is shifted to low values, where very small charges are cut by the applied signal threshold. . . . . . . . . . . . .

7.21 In-pixel efficiency for device FBK13 (full 3D, unirradiated) at perpendicular incidence, folded into one pixel and half of its neighbours. The 3D pixel cell structure is drawn

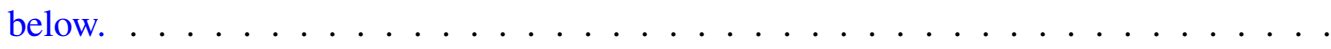

7.22 Average hit efficiencies of unirradiated full 3D devices at different signal thresholds and

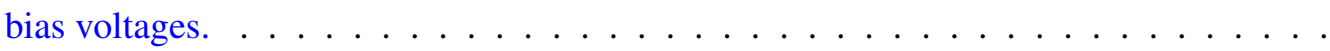

7.23 Average hit efficiencies of irradiated full 3D devices for a set of voltages and thresholds. 116

7.24 Hitmap of device FBK11 during June 2012 testbeam. Most of the sensor area is masked. 116 
7.25 Stave layout for IBL, showing planar double chip sensors in the central $75 \%$ of the stave and 3D sensors in the stave extremities. The design includes fixed gaps of $205 \mu \mathrm{m}$ between neighbouring modules. Taken from [46] and coloured for better visibility. . .

7.26 Photograph of the fully assembled IBL detector on a support structure, including services. The staves are protected by a plastic cover, which is kept in place by three metal clamps. Picture taken by H. Pernegger. . . . . . . . . . . . . . . . . . .

7.27 Efficiency map for a planar double chip (left) and a full 3D device (right) at perpendicular incidence. A signal threshold of $3000 \mathrm{e}^{-}$was applied to both devices and bias voltages of $100 \mathrm{~V}$ for the planar device and $25 \mathrm{~V}$ for the full 3D. Both devices show a homogeneous and high efficiency over the whole sensor. . . . . . . . . . . . .

7.28 Average detected cluster ToT value, depending on the position on the sensor. The planar double chip is shown on the left, the full 3D device on the right. For both devices the scintillator area is clearly visible. . . . . . . . . . . . . . . .

7.29 Average time between a trigger signal is sent and a matched cluster is detected in a device in units of $25 \mathrm{~ns}$ for a planar double chip device (left) and a full 3D device (right) at a threshold of $3000 \mathrm{e}^{-}$. This is a result of slightly different feedback tuning of the two FEs.

7.30 Efficiency map for a planar double chip (left) and a full 3D device (right), rotated by $15^{\circ}$ along $\phi$-direction. The signal threshold was set to $2000 \mathrm{e}^{-}$, the bias voltages were $100 \mathrm{~V}$ for the planar device and $25 \mathrm{~V}$ for the full $3 \mathrm{D} . \ldots \ldots \ldots$

7.31 Average hit efficiency as a function of the run number for the planar device (left) and 3D device (right). The data taking problems, which influenced the 3D device in some runs, can be clearly seen in the calculated efficiency. . . . . . . . . . . . . . .

7.32 Hit efficiency for the pixels in the central double column of the double chip sensor. Top: measurement at a threshold of $3000 \mathrm{e}^{-}$and perpendicular incidence. Bottom: measurement at a threshold of $2000 \mathrm{e}^{-}$, device rotated by $15^{\circ}$. The bias voltage was $100 \mathrm{~V}$ for both measurements. For both measurements the preliminary hit efficiency is

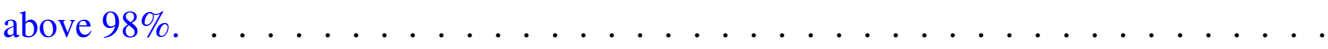

7.33 Second set of measurements at a rotation of $15^{\circ}$, threshold of $2000 \mathrm{e}^{-}$and bias voltages of $100 \mathrm{~V}$ for the planar device (left) and $25 \mathrm{~V}$ for the full 3D (right). The previous data taking problem was solved and both devices show the expected high efficiencies over the whole region, covered by the scintillators. . . . . . . . . . . . .

7.34 Efficiency measurement at a rotation of $15^{\circ}$, threshold of $3000 \mathrm{e}^{-}$and bias voltages of $100 \mathrm{~V}$ for the planar device (left) and $20 \mathrm{~V}$ for the double sided 3D (right). . . . . . . .

7.35 Raw hitmaps for double chip 92-23-01 (left) and double sided 3D device C60-20-03 (right). The regions free of hits correspond to the empty areas in the efficiency maps. .

7.36 Efficiency maps for the investigated 4-chip device (left) and the reference device (right). The 4-chip device shows a homogeneous efficiency with a small horizontal gap in the region, where the lower double chip ends and the upper double chip begins. In the efficiency map of the reference device the same gap can be seen around row 150 in full size. . . . . . . . . . . . . . . . . . . . .

7.37 Average delay between a trigger signal is sent and a matched cluster is detected in a device in units of $25 \mathrm{~ns}$. Left: the planar 4-chip device shows different average response times, depending on the FE chip in which the signal is processed. Right: the reference single chip device shows a homogeneous response time. Both devices were tuned to a threshold of $3200 \mathrm{e}^{-} \ldots \ldots \ldots \ldots \ldots \ldots \ldots$ 
A.1 Section of TBmonII main config, showing the available settings for one DUT. . . . . . 134 



\section{List of Tables}

2.1 Comparison of the expexted increase of significance for three exemplary resonance masses and coupling values $g$. The expectation is to have collected an integrated luminosity of $300 \mathrm{fb}^{-1}$ until the long shutdown for the HL-LHC upgrade begins (left column). After additional 10 years of running the HL-LHC $3000 \mathrm{fb}^{-1}$ are expected in total (right column) . . . . . . . . . . . . . . . . . .

3.1 Characteristic values for different incident radiation in silicon. All particles are supposed to have an energy of $1 \mathrm{MeV}$. The values are $R_{\max }$ as the maximum possible recoil energy of the incident particle, $R_{a v}$ giving the average recoil energy and $E_{\min }$ point defect the minimum energy that is required to create a point defect in the lattice [28] . . . . .

4.1 Benchmark data for the planned pixel detector in the context of the upgrade towards the High Luminosity LHC [43]. . . . . . . . . . . . . . . . . . . .

4.2 Summary of the geometrical parameters for pixel modules to be built for the ATLAS Insertable b-Layer $[46] \ldots \ldots \ldots \ldots \ldots \ldots$

6.1 BeamProfile: overview of the available cuts and produced histograms. . . . . . . . . 78

6.2 ClusterChecker: overview of the available cuts and produced histograms. . . . . . . 79

6.3 Efficiency: overview of the available cuts and produced histograms. . . . . . . . . . 82

6.4 QEfficiency: overview of the available cuts and produced histograms. . . . . . . . . 84

6.5 QShare2D: overview of the available cuts and produced histograms. . . . . . . . . 85

6.6 SumToT: overview of the available cuts and produced histograms. . . . . . . . . . . 87

7.1 Investigated devices of this section. For irradiation fluences specified as $\geq 5 \cdot 10^{15} \frac{\mathrm{n}_{\mathrm{eq}}}{\mathrm{cm}^{2}}$, the radiation monitors in the irradiation facilities did not work properly. Thus, the total gained fluence was higher than demanded, but could not be determined exactly. . . . .

7.2 Bias voltages, leakage currents, average efficiencies and tunings of planar $250 \mu \mathrm{m}$ thick SE sensors. . . . . . . . . . . . . . . . . . . . . 102

7.3 Bias voltages, leakage currents, average efficiencies and tunings of planar $200 \mu \mathrm{m}$ thick SE sensors. The measurement at $60 \mathrm{~V}$ bias voltage was performed in the magnetic field of the Morpurgo magnet. . . . . . . . . . . . . . . . . . . .

7.4 Bias voltages, leakage currents, average efficiencies and tunings of planar $200 \mu \mathrm{m}$ thick SE sensors after irradiation. The irradiation levels of the measured devices are: SCC60, PS2 and SCC61 $\Rightarrow 5 \cdot 10^{15} \frac{\mathrm{n}_{\mathrm{eq}}}{\mathrm{cm}^{2}}$ and the neutron irradiated LUB4 $\Rightarrow 4 \cdot 10^{15} \frac{\mathrm{n}_{\mathrm{eq}}}{\mathrm{cm}^{2}} . \ldots$.

7.5 Bias voltages, leakage currents, average efficiencies and tunings of irradiated double sided 3D devices. The irradiation level of the measured devices is $5 \cdot 10^{15} \frac{\mathrm{n}_{\mathrm{eq}}}{\mathrm{cm}^{2}} \ldots \ldots$. .

7.6 Bias voltages, leakage currents, average efficiencies and tunings of irradiated full 3D devices. Note: the measurement of FBK11 at the threshold of $1600 \mathrm{e}^{-}$was done at perpendicular incidence, while all other measurements were done for $15^{\circ}$ rotation along the $\phi$-direction. 
7.7 IBL testbeam June 2011, batch 1. The different sensor design types conservative, 3D double sided, full 3D and slim edge (SE) are explained in section 4.2 . . . . . . . . . . 118

7.8 IBL testbeam September 2011, batch $4 \ldots \ldots \ldots \ldots$ 


\title{
Curriculum Vitae
}

\section{Personal Details}

\author{
Name Matthias George \\ Date of Birth 09.03.1984 \\ Email mgeorge@uni-goettingen.de \\ Nationality German
}

\section{Education}

2000-2003 Abitur, Oberstufengymnasium Eschwege, Eschwege, Germany

2003-2009 Diploma in Physics, Georg-August-Universität Göttingen, Göttingen, Germany.

2009-2014 PhD in Physics, Georg-August-Universität Göttingen, Göttingen, Germany.

2011-2012 Research period, University of Milan, Milan, Italy.

\section{Professional Experience}

2011 TRIUMF Summer School 2011, Vancouver, Canada.

$201315^{\text {th }}$ International Workshop on Radiation Imaging Detectors, Paris, France.

\section{Languages}

German Mother tongue

English Fluent

\section{List of Publications}

- J. Weingarten et al., Planar Pixel Sensors for the ATLAS Upgrade: Beam Tests results, JINST 7 (2012) P10028

- The ATLAS IBL collaboration, Prototype ATLAS IBL Modules using the FE-I4A Front-End Readout Chip, JINST 7 (2012) P11010

- R. Nagai et al., Evaluation of novel KEK/HPK n-in-p pixel sensors for ATLAS upgrade with testbeam, Nucl.Instrum.Meth. A699 (2013) 78-83

- M. George, Recent Achievements of the ATLAS Upgrade Planar Pixel Sensors R\&D Project, JINST 9 (2014) C05004

- ATLAS author since 2010 